\title{
Stopping Tumor Necrosis Factor Inhibitors in well controlled Rheumatoid Arthritis patients:
}

THE POET STUDY.

M. Ghiti Moghadam

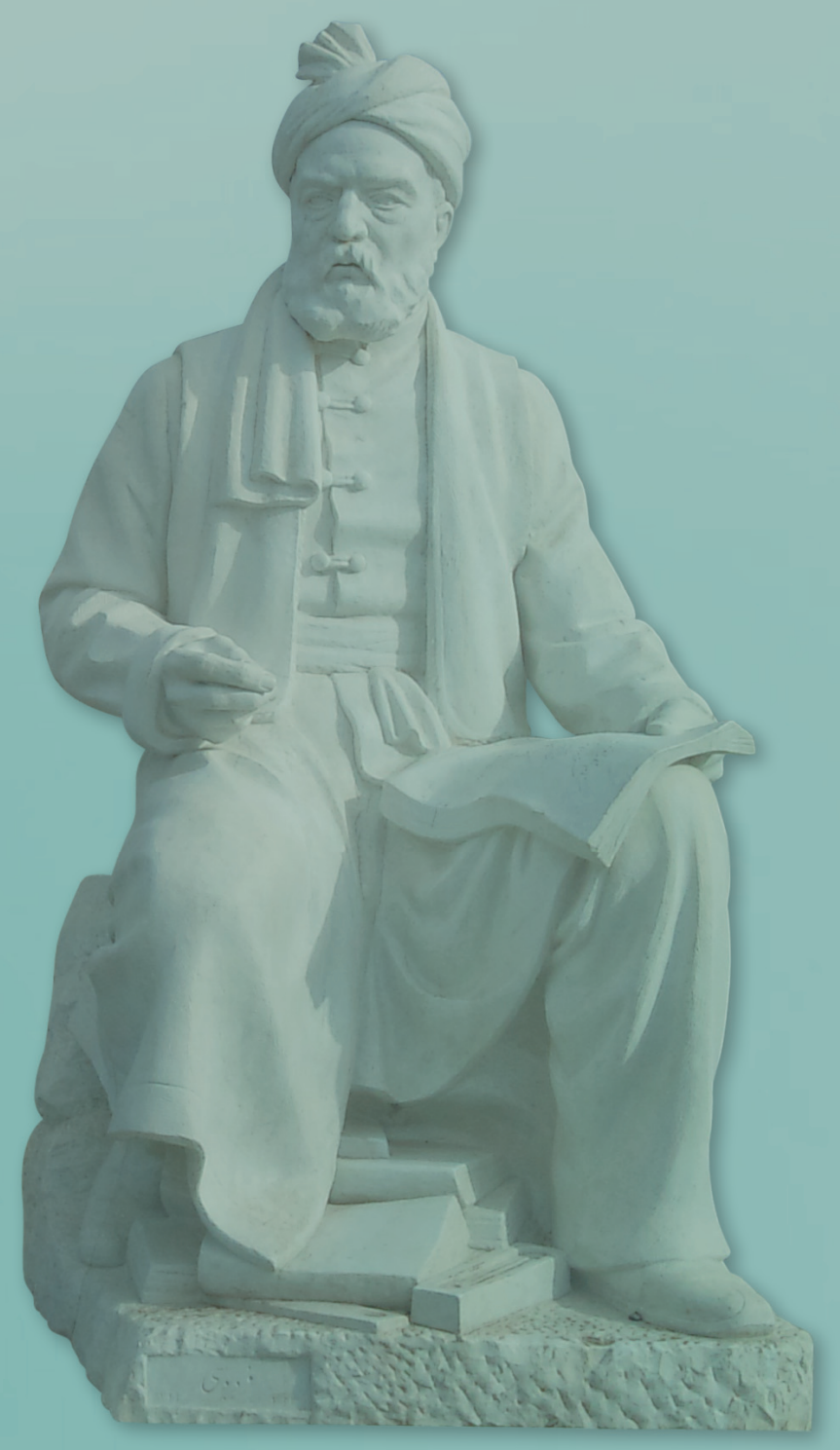




\section{STOPPING TUMOR NECROSIS FACTOR INHIBITORS IN WELL CONTROLLED RHEUMATOID ARTHRITIS PATIENTS: \\ THE POET STUDY}


Dit proefschrift is goedgekeurd door de Promotor Prof. Dr. M.A.F.J. van de Laar en de Co-promotoren dr. H.E. Vonkeman en dr. T.L.C.M. Jansen.

The publication of this disseration was financially supported by Medisch Spectrum Twente, Enschede.

\section{MST}

Cover: $\quad$ Studio ter Avest

Lay-out: $\quad$ Ilse Modder, www.ilsemodder.nl

Print by: $\quad$ Gildeprint - Enschede, www.gildeprint.nl

ISBN: $\quad$ 978-94-6323-548-8

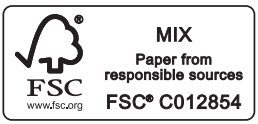

(c) M. Ghiti Moghadam, Twente, the Netherlands, 2019.

All rights reserved. No part of this thesis may be reproduced or transmitted in any form or by any means, electronic or mechanical, including photocopy, recording or any information storage or retrieval system, without prior permission of the author. 


\title{
STOPPING TUMOR NECROSIS FACTOR INHIBITORS IN WELL CONTROLLED RHEUMATOID ARTHRITIS PATIENTS: THE POET STUDY
}

\author{
PROEFSCHRIFT \\ ter verkrijging van \\ de graag van doctor aan de Universiteit Twente, \\ op gezag van de rector magnificus \\ prof. dr. T.T.M. Palstra, \\ volgens besluit van het College voor Promoties \\ in het openbaar te verdedigen \\ op vrijdag 22 maart 2019 om 16:45 uur
}

door

Marjan Ghiti Moghadam

geboren op 3 juli 1969

te Tehran- Iran 


\section{Promotie commissie:}

Voorzitter: Prof. dr. T.A.J. Toonen

\section{Promotor:}

Prof. dr. M.A.F.J. van de Laar (Universiteit Twente)

\section{Copromotoren:}

Dr. H.E. Vonkeman (Universiteit Twente)

Dr. T.L.C.M. Jansen (Radboud Universiteit Nijmegen)

\section{Manuscriptcommissie:}

Prof. dr. R. Westhovens (Katholieke Universiteit Leuven, België)

Prof. dr. P. Verschueren (Katholieke Universiteit Leuven, België)

Prof. dr. W.F. Lems (Vrij Universiteit Amsterdam)

Prof. dr. A.E.R.C.H. Boonen (Universiteit Maastricht)

Prof. dr. ir. H.J. Hermens (Universiteit Twente)

Dr. E. Taal (Universiteit Twente) 


\section{CONTENTS}

$\begin{array}{lll}\text { CHAPTER } 1 \quad \text { General Introduction } & 09\end{array}$

CHAPTER 2 Stopping Tumor Necrosis Factor-inhibitors in Patients with Established 29

Rheumatoid Arthritis in Remission or Stable Low Disease Activity:

A Pragmatic Randomized Multicenter Open-Label Controlled Trial.

CHAPTER 3 Predictors of Biologic-free Disease Control in Patients with Rheumatoid 49

Arthritis after Stopping Tumor Necrosis Factor Inhibitors Treatment

CHAPTER 4 Multi-biomarker Disease Activity Score as a Predictor of Disease

Relapse in Patients with Rheumatoid Arthritis Stopping TNF Inhibitor

Treatment.

CHAPTER 5 Impact of Stopping Tumor Necrosis Factor-inhibitors on Rheumatoid

Arthritis Patients' Burden of Disease.

CHAPTER 6 An Economic Evaluation of Stopping versus Continuing TNF-Inhibitor

Treatment in Rheumatoid Arthritis Patients in Remission or Low Disease Activity: results from the POET randomized trial.

CHAPTER 7 Measuring Disease Exacerbation and Flares in Rheumatoid Arthritis:

Comparison of commonly used Disease Activity Indices \& Individual Measures.

CHAPTER 8 Genaral discussion

Addendum

About the author

Dankwoord

List of publications 

Chapter

1

General introduction 


\section{TNF-INHIBITOR THERAPY IN RHEUMATOID ARTHRITIS}

Rheumatoid arthritis (RA) is a systemic inflammatory disorder and the most common form of inflammatory arthritis. It has a substantial societal impact in terms of cost, disability, and lost productivity. RA signs and symptoms in early disease are driven by inflammation and include joint pain, joint stiffness, and joint swelling. Untreated, the disease can affect many organs throughout the body, but the joints are usually most severely affected. Although there is impressive knowledge on the pathology of RA, the cause of RA remains unknown. Presently, the hypothesis that the combination of a susceptible genetic profile in combination with external factors triggers the onset of the inflammatory disease is widely accepted. The prevalence of RA is estimated around 1\%. The course of the disease is variable if untreated. Approximately $15 \%$ to $20 \%$ of patients have a relatively good prognosis. In other patients, a more progressive disease course is present. In some patients a rapid course of increasing joint damage is seen ${ }^{(1,2)}$.

Several auto-antibodies have been found in RA, especially rheumatoid factor (RF) and anticitrullinated peptide antibodies (ACPA). Although RF is the 'classic' auto-antibody in RA, it can be found also in healthy people and other types of immune mediated diseases. The sensitivity and specificity of RF for RA are up to $70 \%$ and $85 \%$, respectively. ACPA is slightly more specific for RA (sensitivity up to about 70\% and specificity up to 95\%) and thought to play a role in the pathogenesis of the disease ${ }^{(3)}$.

Therapeutic strategies in RA have been changed in the last decades. In the 1970s, it was common to start with Aspirin or non-steroidal anti-inflammatory drugs (NSAIDs). In those days, available disease-modifying anti-rheumatic drugs (DMARDs) were only initiated once radiographic damage had been demonstrated ${ }^{(4)}$. Especially outside Europe, patients were frequently treated with corticosteroids only.

Over the last decades of the last century, conventional DMARDs became available, such as anti-malaria (hydroxychloroquine), sulphasalazine, oral gold, intramuscular gold, azathioprine and methotrexate (MTX). In general, the dose effect relation and the dose toxicity relation were disappointing, resulting in their application being restricted to late in course of the disease. At the turn of the century, treatment strategies changed. DMARD therapy was started earlier in the disease course and sometimes in combination therapy. Some Dutch clinical trials such as the COBRA, DREAM Remission Induction Study and BeSt-study showed that initiation of combination therapy including corticosteroids in an early phase of RA leads to less joint destruction and better outcomes ${ }^{(5-7)}$. 
A major breakthrough for the treatment of RA was the development and introduction of

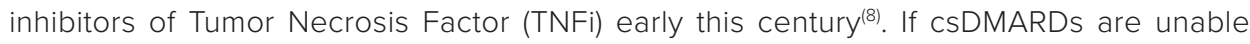
to control RA disease activity, addition of a TNFi or other biological (b)DMARD should be considered ${ }^{(9)}$. Also, it has been demonstrated that early effective treatment in RA is associated with prevention, or at least limitation, of joint damage and long term disability. Suggesting a possible therapeutic "window of opportunity," by starting effective DMARD therapy immediately after making the diagnosis ${ }^{(10,11)}$.

A fundamental improvement in RA treatment strategies was the adoption of the remission induction, or treat-to-target of remission, principle. The underlying principle of treat-totarget (T2T) strategies is that in a specific outcome domain, a measurement instrument and measurement target is defined. Well-known examples of T2T are diabetes mellitus and hypertension treatment. Subsequent use of T2T principles in RA confirmed that treating RA to a target of remission, allows patients to reach better outcomes ${ }^{(12-15)}$. According to the 2016 update of the EULAR recommendations, monitoring should be frequent in early and active disease and if the target is not met at 3-6 months after the start of treatment, therapy should be adjusted ${ }^{(9)}$.

There have been many studies demonstrating the efficacy of adding TNFi to csDMARDs to achieve disease remission ${ }^{(16-18)}$. Whenthe prescription of a TNFi for RA patients results in remission or low disease activity (LDA), however, prescribers and patients generally stay with TNFi indefinitely. This may result in overtreatment, unnecessary side effects, and related costs in some patients ${ }^{(19)}$. TNFi are known to increase the risk of infections and are related to the occurrence of some types of cancer ${ }^{(20-25)}$. Since TNFi's come at a considerable price, reducing or stopping TNFi when the disease course allows to do so, might reduce side effects and costs. However, it is unclear whether patients in remission or stable low disease activity need to continue TNFi indefinitely or can safely and effectively stop this treatment.

\section{A THEORETICAL FRAMEWORK ON TNF-INHIBITOR START, STOPPING AND TAPERING}

The holy grail in medicine is evidence provided by clinical trials. For starting a medical intervention, in this case TNFi for the treatment of RA, this is relatively straightforward. Consequently, numerous pivotal and non-pivotal trials on all TNFi components are available, leaving no room to doubt the efficacy of TNFi for the treatment of RA. Data on stopping or tapering TNFi, however, is relatively scarce and published in both dedicated and nondedicated studies. In order to help to understand the results of these studies, which will be 
reviewed below, it is helpful to discuss a framework based on available clinical knowledge on RA diagnosis, classification, pathology, natural history and treatment.

RA is not a homogenous pathophysiological substrate, but a clinical syndrome or phenotype. Although extremely typical cases can be easily recognized by experienced clinicians, less typical cases may lead to different clinical decisions by experts. Therefore, international classification criteria have been developed and used to facilitate more or less homogenous RA populations, especially for clinical studies. These criteria have, for good reasons, been adapted over time, where the most recent adaptations intended to include less typical cases in order to allow the best possible treatment options for arthritis patients. As we speak, RA populations in studies and clinical care are heterogeneous, probably with different pathological mechanisms and different natural history. For information on the natural history of RA, we have to return to the past century because presently almost all patient are treated with effective anti-rheumatic therapies. Spontaneous sustained remissions of recently developed RA were observed in epidemiological studies, as well as RA patients where the disease activity seemed to burn out after a long-term course, leaving the patients with damage due to the disease.

RA patients are well known to respond differently on all types of anti-rheumatic treatments. Moreover, clinical characteristics and biomarkers show a considerable variability in RA. Although never formally proven to be true, the concept of the window of opportunity is very popular. This theory supposes that in early stages of RA, treatment is more effective than in later stages. Finally, I did not mention the criteria of remission or low disease activity (LDA), used to make the decision to stop or taper TNFi. It does not need any discussion that only true remission will potentially mark the ideal moment of stopping or tapering. However, in RA the debate on the appropriate criteria for remission, from a scientific as well as clinical perspective, is still ongoing.

With respect to stopping or tapering of an effective treatment of RA with TNFi, all considerations mentioned above can theoretically influence the result. In the figure below I summarize these considerations.

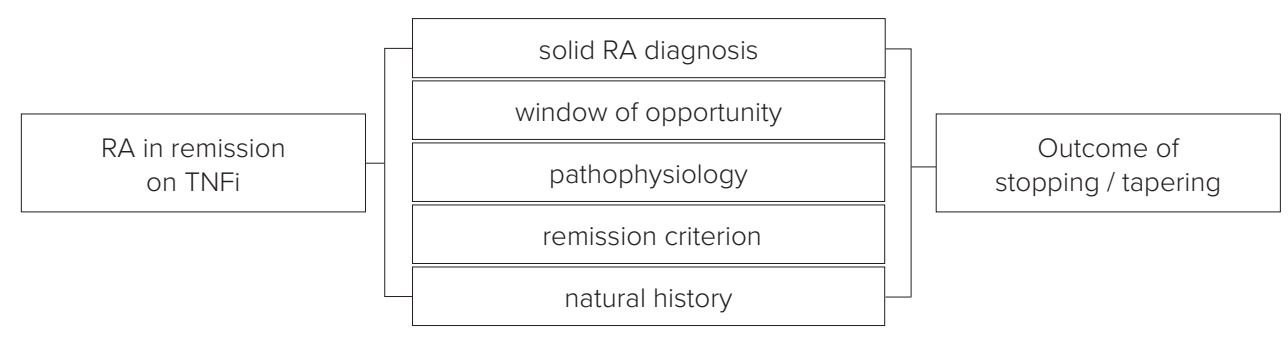




\section{STOPPING OF TNF-INHIBITOR THERAPY}

Some studies have addressed the effects of TNFi tapering or stopping in patients with RA in remission or with LDA on disease activity, physical function, costs, safety and structural damage, compared with continuation of the prescribed standard dose. These studies examined different ways of tapering or stoppingof TNFi(26,27). Hereafter, I will review published studies on stopping or tapering of TNFi in RA per specific TNFi.

\section{ETANERCEPT}

In a 78-week multicenter randomized placebo-controlled superiority trial (the EMPIRE trial), Villeneuve et al.(28) examined the effect of stopping etanercept in early RA patients. Patients had a mean symptom duration of 7 months with a mean baseline DAS28-CRP of 4.22. All patients were DMARD-naive early RA and were either rheumatoid factor, anticitrullinated protein antibodies, or shared epitope positive. Patients were randomized 1:1 to receive MTX + etanercept or MTX + placebo for 52 weeks. Injections of etanercept or placebo were subsequently stopped in all patients at week 52. In those with no tender or swollen joints for $>26$ weeks, injections were stopped early ${ }^{(28)}$. There was no etanercept continuation control arm. After withdrawal of etanercept, $41.9 \%$ remained in sustained remission and $57.7 \%$ remained in LDA.

The PRESERVE trial ${ }^{(29)}$ studied starting etanercept $50 \mathrm{mg}$ weekly as well as stopping or dose reduction ( $25 \mathrm{mg} / \mathrm{wk}$ ) compared with continuation of the full $50 \mathrm{mg}$ weekly dose of etanercept when LDA was reached at 36 weeks. This randomized, double blinded, placebo controlled trial had three arms (1:1:1), with a total follow-up of 88 weeks. The study population consisted of 604 participants using MTX. The study was conducted in 80 centers in Europe, Latin America, Asia and Australia. Most patients were female. Mean age was approximately 47 years. Participants had to have active disease at baseline $(3.2<$ DAS $28<5.2)$ to enter the open treatment with etanercept. After reachingDAS $28 \leq 3.2$ at week 36, patients who were still using MTX were randomized. After one year, $42.6 \%$ of the patients who had stopped taking etanercept still had DAS28 $\leq 3.2$, compared with $82.6 \%$ in the full-dose group and $79.1 \%$ in the half-dose group. These results are in line with the EMPIRE trial(30). The PRESERVE study suggests that some RA patients with extensive disease duration are able to stop or reduce the dose of etanercept $(29,31)$.

In the DOSERA study, a European randomized placebo-controlled trial, 73 patients diagnosed with RA received etanercept $50 \mathrm{mg}$ (in one single or two divided weekly doses) for at least 14 months in combination with MTX at a stable dose of 7.5-25 mg/week ${ }^{(32)}$. Patients who had a DAS28 $\leq 3.2$ were randomized to one of three arms (period 2): etanercept 
50 mg weekly + MTX (unchanged:ETN50); etanercept 25 mg weekly + MTX (reduced dose: ETN25), or placebo + MTX (stop: PBO). If a flare occurred after randomization, the patient was withdrawn. Only 13\% of those who discontinued etanercept (PBO group) remained free of flares during 48 weeks observation. The proportion of patients remaining free of flare was significantly higher in both other groups: ETN50 (52\%; p=0.007) and ETN25 (44\%; $p=0.044)$ versus PBO (13\%).

In sum, studies on tapering and stopping ETA in RA patients in remission suggest that tapering is an option for many patients, but stopping for only a few.

\section{ADALIMUMAB}

Some studies have examined the effects of stopping or tapering adalimumab in RA. In the OPTIMA trial, 1032 MTX-naïve early RA patients were randomized to adalimumab plus MTX or placebo plus MTX ${ }^{(33)}$. Patients in the adalimumab plus MTX group who achieved stable Iow disease activity (DAS28-CRP $<3.2$ at weeks 22-26) were randomized to adalimumabcontinuation or adalimumab-stop where MTX was continued. The majority of patients in the adalimumab-stop group maintained their good responses, while in $44 \%$ of patients stopping adalimumab resulted in loss of the status of remission or LDA.

HIT HARD was a double-blind, randomized controlled trial, designed to compare two treatment strategies in DMARD-naive patients with early $\mathrm{RA}^{(34)}$. Eligible patients were aged 18-75 years, with a disease duration of up to 1 year. No current or prior therapy with DMARDs or biologics was allowed. All patients started MTX (open label subcutaneous MTX weekly (15 mg/week)) and were randomized to adalimumab (ADA) $40 \mathrm{mg}$ subcutaneously every other week or placebo (PBO) subcutaneously every other week over 24 weeks. After 24 weeks all patients continued MTX, but ADA and PBO injections were stopped. The primary endpoint of the HIT HARD study was the DAS28 score at week 48. At week 24, initial treatment with ADA/MTX compared with $\mathrm{PBO} / \mathrm{MTX}$ resulted in a greater reduction in DAS28 and other secondary outcomes such as DAS28 remission and HAQ. However, at week 48, the difference in clinical outcomes between groups was not statistically significant. The results of this study suggest that starting adalimumab in all early RA patients in addition to MTX $15 \mathrm{mg}$ benefits the clinical outcomes after 24 weeks, an effect that is lost after 48 weeks after stopping $\mathrm{TNFi}^{(34)}$.

The open-label, non-randomized, HONOR trial included 197 patients with early RA and moderate-to-severe disease activity (DAS28-ESR $\geq 3.2)^{(35)}$. The mean disease duration was 7.5 years and most patients had active disease despite MTX, with a mean DAS28-ESR of 5.1 at baseline. Thirty-five percent of the patients reached DAS28 remission at week 24 . 
Fifty-one out of these sixty-nine patients in remission stopped ADA. Fifty-eight percent of remission patients that stopped ADA stayed in remission. Sustained remission was predicted by low DAS28 at baseline. In contrast, in the smaller retrospective BRIGHT study, a Japanese 52-week study in 46 RA patients in remissionor LDA (DAS28<2.7) on ADA monotherapy, 24 patients continued ADA, while 22 stopped ADA. Only four out of the 22 stoppers maintained a low DAS28 at week $52^{(36)}$.

In summary, the few published studies on stopping adalimumab in RA patients are different with respect to size and different methodology. However, stopping ADA in patients being in stable remission, with background medication of MTX, seems an option.

\section{INFLIXIMAB}

One of the first publications on stopping TNFi by Quinn et al. ${ }^{(37)}$ in 2005 , described a small group of early RA patients, treated with infliximab plus MTX, in whom infliximab was stopped. After stopping infliximab, 70\% showed sustained remission. This observational study was small and did not include a control group of infliximab continuation.

The Dutch RA strategy study (BeSt study) contained an arm with early infliximab treatment. In the other arms, TNFi was started late when csDMARDs proved to be unable to obtain remission. In all patients using infliximab, the treatment was stopped in case of sustained remission. Sixty-four percent of patients who started early with infliximab, were able to stop their infliximab, versus only $25 \%$ of those who started later ${ }^{(38)}$.

The Remission induction by Remicade study in patients with RA was a Japanese multicenter prospective study in patients with established RA (mean disease duration of 5.9 years), treated with infliximab. Infliximab was stopped when patients achieved LDA, defined as DAS28 $<3.2$ for 24 weeks. After stopping, $50 \%$ of patients remained in low disease activity. This study demonstrated that patients with long disease duration and with concurrent MTX therapy can also try to stop TNFi after reaching remission or LDA ${ }^{(39)}$.

Van der Maas et al. ${ }^{(40)}$ performed an observational study on the option of tapering or downtitration until stopping of infliximab after 6 months of LDA. Infliximab dose was tapered with 25\% every 8-12 weeks until flare or stopping based upon the results of the DAS28 assessments. After 1 year of tapering, 16\% stopped infliximab and $45 \%$ could be down-titrated.

In summary, the reviewed studies on stopping infliximab suggest that stopping infliximab in RA patients in remission is an option, whereas dose optimization is realistic in many of these patients. 


\section{CERTOLIZUMAB PEGOL}

In a small study comparable to the above cited HONOR study, certolizumab pegol was started and after 24 weeks stopped as an add-on in RA patients with active disease on cSDMARDs. Certolizumab could only be stopped effectively in a very limited number of RA patients, but restart of this TNFi was effective in case of failure to stop ${ }^{(41)}$. This study suggests that stopping of certolizumab pegol in case of remission is not likely to be a good idea. However, there is only one study with a limited number of patients.

\section{GOLIMUMAB}

So far, there are no published trials examining the effects of stopping or tapering golimumab in RA patients.

\section{MISCELLANEOUS}

The reviewed studies above addressed stopping or tapering of specific TNFi's. However, the effect of stopping of miscellaneous TNFi was addressed in other studies. Saleem et al. ${ }^{(42)}$ studied patients on etanercept, adalimumab, or infliximab. In this study, 47 RA using TNFi plus MTX (some patients with initial treatment with TNFi, others with treatment with TNFi started later in the disease) stopped TNFi treatment. All patients were in DAS28 remission and with absent signs of inflammation on power doppler ultrasound. Two years after stopping TNFi, sustained remission was seen in 59\% of patients with initial TNFI treatment, as compared with $15 \%$ in the patients who started TNFi later in the disease.

Brocq et al. ${ }^{(43)}$ examined the relapse rate after stopping TNFi in patients with LDA defined as DAS $<2.6$ for at least 6 months. Twelve months after stopping, 25\% patients were still in remission. Predictors of drug-free remission were long-term use of TNFi and longstanding remission. In the Corrona registry, an analysis in 717 patients with long standing RA with low disease activity showed that $73.4 \%$ of patients had a favorable disease course for more than 12 months after stopping their TNFi(44).

Although the studies reviewed above on stopping TNFi are difficult to compare due to differences in methods and populations studied, stopping TNFi without an immediate flare seems realistic for a selection of patients. 


\section{TAPERING OF TNF-INHIBITOR THERAPY}

In contrast to studies stopping TNFi at once, some of the studies mentioned above examined the effects of TNFi tapering or dose reduction as well. In addition to the DOSERA and PRESERVE studies described above ${ }^{(29,31,32)}$, two other randomized controlled trials ${ }^{(45,46)}$ compared etanercept tapering versus continuation. Al four studies indicate that tapering resulted in an small increased flare risk.

In the DRESS study performed in two Dutch clinics was published by van Herwaarden et al. ${ }^{(47)}$, 180 RA patients in remission on TNFi SC (adalimumab or etanercept) were randomized to tapering or continuing TNFi SC (2:1). Every 3 months the dosing was adapted according to the DAS28 score. Twenty percent of patients were able to stop TNFi, 43\% were able to taper dose of TNFi and 37\% had to continue the original dose of TNFi. Although numerically increased disease activity was related to tapering TNFi, the difference in flare was noninferior versus continuation of $\mathrm{TNFi}^{(47)}$.

The RETRO study was a multicenter, randomized, open, prospective, controlled, parallelgroup trial. The authors evaluated the possibility of tapering or stopping anti-rheumatic therapy including TNFi in RA patients with stable remission (DAS28-ESR $<2.6$ for at least 6 months). One hundred one patients using cSDMARDs or bDMARDs were randomized into three different trial arms: continuation, tapering the dose in two six months steps, or to stopping of all csDMARDS and/or bDMARDs. Disease relapse rate was $15.8 \%$ in the continuation group, $38.9 \%$ in the tapering group, and $51.9 \%$ in the stop group. The difference in disease relapse between the tapering and the stop group was not statistically significant ${ }^{(48)}$.

Although data are still limited, and methodological differences make interpretation difficult, the reviewed studies on tapering suggest that in case of remission, TNFi tapering by decreased dose or increased dosing interval is feasible with an acceptable increase of disease activity including flare rates. In comparison with immediate stopping of TNFi, as reviewed earlier, tapering show numerically less flares and less increased disease activity. However, pro's and con's for tapering and stopping are rarely mentioned. For instance, decreased dosing and increasing dosing intervals both are, in contrast with stopping, offlabel and potentially come with increased immunogenicity. In accordance to the framework discussed before, data from the reviewed studies suggest that remission obtained early in the disease course and a remission without any signs of residual disease activity are related to better results, supporting the window of opportunity theory and the stringent definition of remission, respectively. 


\section{PREDICTORS OF SUCCESFUL STOPPING/TAPERING OF TNF-INHIBITORS}

Several studies have tried to identify predictors of successful stopping of TNFi. Although these studies have been performed as part of the studies reviewed above, the methodological problems are even more challenging. Firstly, shorter disease duration, when obtaining remission was found to be a predictor for successful stopping in some studies $^{(39,42,48)}$. Low disease activity score and low erosion score at baseline were in some studies related to successful TNFi stopping/tapering ${ }^{(39,44,48)}$. With respect to biomarkers, anticitrullinated peptide antibodies (ACPA) was found as a predictor in the RETRO study, the BesT study and the study by Haschka et al. ${ }^{(48,49)}$. Rheumatoid factor positivity was related to loss of clinical benefit in Corrona study ${ }^{(44)}$. Several other predictors of successful stopping/ tapering that have been found in single studies are non-smoking ${ }^{(49)}$, younger age ${ }^{(39)}$, lower VAS pain ${ }^{(32)}$ and higher body mass index (BMI) ${ }^{(44)}$. Duration of TNFi treatment as well as early initial TNFi treatment showed conflicting results ${ }^{(42,48)}$. Finally, Smolen et al. ${ }^{(31)}$ suggested that low CRP levels after achieving low disease activity (DAS28<3.2) are indicative of increased possibility of successful adalimumab-free disease control.

The potential difference between types of $\mathrm{TNFi}^{(50-53)}$ as a predictor of stopping or tapering has not been sufficiently analyzed or reported in the reviewed publications. The results from the previous studies and conclusions might also not be directly applicable in daily clinical practice, because more predictors for successful results are needed for clinicians and patients to help them make the stopping or tapering decision.

Moreover, the reviewed studies have generally focused on conventional clinical measures of disease activity and traditional biomarkers as potential predictors. In the last few years, the multi-biomarker disease activity (MBDA), containing 12 different serum proteins, has been clinically validated as a measure of disease activity in patients with RA(54-56). Recently, MBDA scores were shown to improve the prediction of relapses in RA patients in stable remission undergoing csDMARD tapering, especially when combined with ACPA testing ${ }^{(57)}$. Further research is needed to determine whether MBDA scores may also be useful in prediction of successful TNFi stopping/tapering. 


\section{THE PATIENT'S PERSPECTIVE - PATIENT REPORTED OUTCOME MEASURES (PROMs)}

Current studies on stopping TNFi have primarily focused on the effects of stopping/tapering on the biomedical domain, i.e., the disease activity status as the primary endpoint. However, RA disease activity, although a driver of the health status of RA patients, may not adequately reflect the patient's perspective since stopping/tapering comes with negative as well as positive emotions and TNFi might have additional direct effects on outcomes that matter to patients, like pain and fatigue, that can only be measured by PROMs. The consequence of such a discordant viewpoint with regard to disease activity, is that decisions are often prone to not being shared between patients and physicians.

Sanderson et al. ${ }^{(58)}$ showed the potential patient mismatch between perceptions of disability and actual disability, and also of patient discrepancy between perceived change and actual change in outcomes. The importance of different perceptions of disease activity in patient and physician has already been shown by Studenic et al. ${ }^{(59)}$. Therefore, changes in clinical measures of disease activity may not adequately reflect the perception of illness and symptoms of RA patients themselves ${ }^{(60)}$. For example, Smolen et al. ${ }^{(31)}$ showed that the mean Health Assessment Questionnaire disability index at 52 weeks follow-up was not statistically significantly different between the etanercept dose reduction group and the etanercept continuation group. Likewise, mean EuroQoL-5D scores after 52 weeks follow-up were equal in the etanercept dose reduction group and the etanercept continuation group.

It does not need additional explanation that the perceived benefit or disadvantage by patients due to stopping/tapering TNFi in case of remission needs to be thoroughly researched. This requires homogenous large studies to provide reliable results that are clinical relevant to guide prescribers and patients in their shared decisions.

\section{HEALTH ECONOMETRICS AND COST-EFFECTIVENESS}

The goals in the treatment of RA are to decrease and control the inflammatory disease activity, to control signs and symptoms like pain and fatigue, and to improve the ability of the patient to fully participate, without or with limited adverse events ${ }^{(61,62)}$. Biological DMARDs, including TNFi's, have considerably improved the outcomes of RA ${ }^{(21)}$. However, the treatment with bDMARDs comes with a considerable price. Stopping or tapering of bDMARDs can reduce healthcare expenditures, as long as the health of patients is jeopardized. Potential savings resulting from safely and effectively stopping treatment with 
biological DMARDs could be used to reinvest in other treatments or to increase the created value. The reviewed publications showed a wide range of patients being free from flare after stopping of TNFi, ranging from 13\% to $80 \%$ after 12 months in the different studies, allowing the possibility that costs of flares and restarting TNFi requires extra resources.

In 2017, Aletaha et al. ${ }^{(63)}$ published a health economical Markov model with a one month's cycle predicting the 5-year clinical and cost impact of different TNFi treatments (adalimumab, etanercept, or infliximab). The model compared continuation versus dose tapering/stopping based upon DAS28 scores. Data were obtained from 14 studies with in total 2309 patients. The authors concluded that both tapering and stopping of TNFi results in costs reduction, but decreased control of disease activity as compared to continuation of the effective dose. 


\section{AIM OF THIS THESIS}

It is beyond any doubt that the introduction of TNFi's early this century really made a difference for RA patients. In the past century, care was the most important objective of healthcare systems for RA patients. After the introduction of bDMARDs, in this case TNFi, cure has become a realistic option for our patients. Having said this, it is striking that overwhelming evidence is available on starting bDMARDs in RA, whereas studies on tapering and stopping in case of treatment success are limited to small investigatorinitiated studies or secondary outcomes in other studies.

I am very grateful to the Dutch Society for Rheumatology that initiated the POET study and the Dutch secretary of Health (ZONMW) that made this study possible. The aim of the POET study was to perform a homogeneous large study that would enable us to address the following important questions that remained unanswered by the preceding studies:

1. Can TNFi be safely stopped when RA patients are in stable remission? (chapter 2);

2. What are the effects of stopping TNFi in RA patients in stable remission according to the patient's perspective? (chapter 3);

3. Is the multidimensional biomarker set (MBDA) a predictor of successfully stopping TNFi in RA patients in stable remission? (chapter 4);

4. Are there clinically relevant predictors of effectively/safely stopping TNFi in RA patients in stable remission? (chapter 5);

5. What are the health economic consequences of stopping versus maintaining TNFi in RA patients in stable remission? (chapter 6). 


\section{REFERENCES}

1. Lee DM, Weinblatt ME. Rheumatoid arthritis. Lancet (London, England). 2001;358(9285):903-11.

2. Scott DL, Steer S. The course of established rheumatoid arthritis. Best Pract Res Clin Rheumatol. 2007;21(5):943-67.

3. Nishimura K, Sugiyama D, Kogata Y, Tsuji G, Nakazawa T, Kawano S, et al. Metaanalysis: diagnostic accuracy of anticyclic citrullinated peptide antibody and rheumatoid factor for rheumatoid arthritis. Ann Intern Med. 2007;146(11):797-808.

4. van Gestel AM, Haagsma CJ, Furst DE, van Riel PL. Treatment of early rheumatoid arthritis patients with slow-acting antirheumatic drugs (SAARDs). Baillieres Clin Rheumatol. 1997;11(1):65-82.

5. Landewé RBM, Boers M, Verhoeven AC, Westhovens R, van de Laar MAFJ, Markusse HM, et al. COBRA combination therapy in patients with early rheumatoid arthritis: long-term structural benefits of a brief intervention. Arthritis Rheum. 2002;46(2):347-56.

6. Schipper LG, Kievit W, den Broeder AA, van der Laar MA, Adang EMM, Fransen J, et al. Treatment strategies aiming at remission in early rheumatoid arthritis patients: starting with methotrexate monotherapy is cost-effective. Rheumatology (Oxford). 2011;50(7):1320-30.

7. Goekoop-Ruiterman YPM, de VriesBouwstra JK, Allaart CF, van Zeben D, Kerstens PJSM, Hazes JMW, et al. Clinical and radiographic outcomes of four different treatment strategies in patients with early rheumatoid arthritis (the BeSt study): a randomized, controlled trial. Arthritis Rheum. 2005;52(11):3381-90.

8. Lipsky PE, van der Heijde DM, St Clair EW, Furst DE, Breedveld FC, Kalden JR, et al. Infliximab and methotrexate in the treatment of rheumatoid arthritis. AntiTumor Necrosis Factor Trial in Rheumatoid Arthritis with Concomitant Therapy Study Group. N Engl J Med. 2000;343(22):1594602.

9. Smolen JS, Landewé R, Bijlsma J, Burmester G, Chatzidionysiou K, Dougados $M$, et al. EULAR recommendations for the management of rheumatoid arthritis with synthetic and biological disease-modifying antirheumatic drugs: 2016 update. Ann Rheum Dis. 2017;76(6):960-77.

10. Quinn MA, Emery P. Window of opportunity in early rheumatoid arthritis: possibility of altering the disease process with early intervention. Clin Exp Rheumatol. 2003;21(5 Suppl 31):S154-7.

11. Boers M. Understanding the window of opportunity concept in early rheumatoid arthritis. Arthritis Rheum. 2003;48(7):1771-4.

12. Steunebrink LMM, Vonkeman HE, Ten Klooster PM, Hoekstra M, van Riel PLCM, van de Laar MAFJ. Recently diagnosed rheumatoid arthritis patients benefit from a treat-to-target strategy: results from the DREAM registry. Clin Rheumatol. 2016;35(3):609-15.

13. Steunebrink LMM, Versteeg GA, Vonkeman HE, ten Klooster PM, Kuper HH, Zijlstra TR, et al. Initial combination therapy versus 
step-up therapy in treatment to the target of remission in daily clinical practice in early rheumatoid arthritis patients: results from the DREAM registry. Arthritis Res Ther. 2016;18(1):60.

14. Versteeg GA, Steunebrink LMM, Vonkeman $\mathrm{HE}$, ten Klooster PM, van der Bijl AE, van de Laar MAFJ. Long-term disease and patientreported outcomes of a continuous treatto-target approach in patients with early rheumatoid arthritis in daily clinical practice. Clin Rheumatol. 2018;37(5):1189-97.

15. Stoffer MA, Schoels MM, Smolen JS, Aletaha D, Breedveld FC, Burmester G, et al. Evidence for treating rheumatoid arthritis to target: results of a systematic literature search update. Ann Rheum Dis. 2016;75(1):16-22.

16. Hochberg MC, Tracy JK, Hawkins-Holt M, Flores $\mathrm{RH}$. Comparison of the efficacy of the tumour necrosis factor alpha blocking agents adalimumab, etanercept, and infliximab when added to methotrexate in patients with active rheumatoid arthritis. Ann Rheum Dis. 2003 Nov;62 Suppl 2:ii136.

17. Goekoop-Ruiterman YPM, de VriesBouwstra JK, Allaart CF, van Zeben D, Kerstens PJSM, Hazes JMW, et al. Clinical and radiographic outcomes of four different treatment strategies in patients with early rheumatoid arthritis (the BeSt study): a randomized, controlled trial. Arthritis Rheum. 2005;52(11):3381-90.

18. Chen Y-F, Jobanputra P, Barton P, Jowett S, Bryan S, Clark W, et al. A systematic review of the effectiveness of adalimumab, etanercept and infliximab for the treatment of rheumatoid arthritis in adults and an economic evaluation of their costeffectiveness. Health Technol Assess. 2006;10(42):1-229.

19. van Ingen ILA, Lamers-Karnebeek $F$, Jansen TL. Optimizing the expediency of TNFi in rheumatoid arthritis: offering a TNFi holiday in patients having reached lowdisease activity in the maintenance phase. Expert Opin Biol Ther. 2014;14(12):1761-7.

20. Listing J, Strangfeld A, Kary S, Rau R, von Hinueber $U$, Stoyanova-Scholz M, et al. Infections in patients with rheumatoid arthritis treated with biologic agents. Arthritis Rheum. 2005;52(11):3403-12.

21. Bongartz $T$, Sutton $A J$, Sweeting $M J$, Buchan I, Matteson EL, Montori V. AntiTNF antibody therapy in rheumatoid arthritis and the risk of serious infections and malignancies: systematic review and meta-analysis of rare harmful effects in randomized controlled trials. JAMA. 2006;295(19):2275-85.

22. Matteson EL, Bongartz T. Tumor necrosis factor antagonists and cancer in patients with rheumatoid arthritis. Nat Clin Pract Rheumatol. 2007;3(1):14-5.

23. Askling J, Bongartz T. Malignancy and biologic therapy in rheumatoid arthritis. Curr Opin Rheumatol. 2008;20(3):334-9.

24. Leombruno JP, Einarson TR, Keystone EC. The safety of anti-tumour necrosis factor treatments in rheumatoid arthritis: meta and exposure-adjusted pooled analyses 
of serious adverse events. Ann Rheum Dis. 2009;68(7):1136-45.

25. van Vollenhoven RF. How to dose infliximab in rheumatoid arthritis: new data on a serious issue. Ann Rheum Dis. 2009;68(8):1237-9.

26. Fautrel B, Pham T, Alfaiate T, Gandjbakhch F, Foltz V, Morel J, et al. Step-down strategy of spacing TNF-blocker injections for established rheumatoid arthritis in remission: results of the multicentre non-inferiority randomised open-label controlled trial (STRASS: Spacing of TNFblocker injections in Rheumatoid ArthritiS St. Ann Rheum Dis. 2016;75(1):59-67.

27. Kievit W, van Herwaarden $N$, van den Hoogen FH, van Vollenhoven RF, Bijlsma JW, van den Bemt BJ, et al. Disease activityguided dose optimisation of adalimumab and etanercept is a cost-effective strategy compared with non-tapering tight control rheumatoid arthritis care: analyses of the DRESS study. Ann Rheum Dis. 2016;75(11):1939-44.

28. Villeneuve E, Nam JL, Hensor E, Wakefield RJ, Conaghan PG, Green MJ, et al. Preliminary results of a multicentre randomised controlled trial of etanercept and methotrexate to induce remission in patients with newly diagnosed inflammatory arthritis. Arthritis Rheum. 2011;63:S960-1.

29. Pavelka K, Szekanecz Z, Damjanov N, Majdan M, Nasonov E, Mazurov V, et al. Induction of response with etanerceptmethotrexate therapy in patients with moderately active rheumatoid arthritis in Central and Eastern Europe in the PRESERVE study. Clin Rheumatol. 2013;32(9):1275-81.

30. Nam JL, Villeneuve E, Hensor EMA, Wakefield RJ, Conaghan PG, Green MJ, et al. A randomised controlled trial of etanercept and methotrexate to induce remission in early inflammatory arthritis: the EMPIRE trial. Ann Rheum Dis. 2014;73(6):1027-36.

31. Smolen JS, Nash P, Durez P, Hall S, Ilivanova E, Irazoque-Palazuelos $F$, et al. Maintenance, reduction, or withdrawal of etanercept after treatment with etanercept and methotrexate in patients with moderate rheumatoid arthritis (PRESERVE): a randomised controlled trial. Lancet. 2013;381(9870):918-29.

32. van Vollenhoven RF, Ostergaard $M$, Leirisalo-Repo $M$, Uhlig $T$, Jansson $M$, Larsson E, et al. Full dose, reduced dose or discontinuation of etanercept in rheumatoid arthritis. Ann Rheum Dis. 2016;75(1):52-8.

33. Smolen JS, Emery P, Fleischmann R, van Vollenhoven RF, Pavelka K, Durez P, et al. Adjustment of therapy in rheumatoid arthritis on the basis of achievement of stable low disease activity with adalimumab plus methotrexate or methotrexate alone: the randomised controlled OPTIMA trial. Lancet. 2014;383(9914):321-32.

34. Detert J, Bastian $H$, Listing J, Weiß A, Wassenberg S, Liebhaber A, et al. Induction therapy with adalimumab plus methotrexate for 24 weeks followed by methotrexate monotherapy up to week 48 versus methotrexate therapy alone for DMARD- 
naive patients with early rheumatoid arthritis: HIT HARD, an investigator-initiated study. Ann Rheum Dis. 2013;72(6):844-50.

35. Hirata S, Saito K, Kubo S, Fukuyo S, Mizuno Y, Iwata S, et al. Discontinuation of adalimumab after attaining disease activity score 28-erythrocyte sedimentation rate remission in patients with rheumatoid arthritis (HONOR study): an observational study. Arthritis Res Ther. 2013 Jan;15(5):R135.

36. Harigai M, Takeuchi T, Tanaka Y, Matsubara T, Yamanaka H, Miyasaka N. Discontinuation of adalimumab treatment in rheumatoid arthritis patients after achieving low disease activity. Mod Rheumatol. 2012;22(6):81422.

37. Quinn MA, Conaghan PG, O'Connor PJ, Karim Z, Greenstein A, Brown A, et al. Very early treatment with infliximab in addition to methotrexate in early, poor-prognosis rheumatoid arthritis reduces magnetic resonance imaging evidence of synovitis and damage, with sustained benefit after infliximab withdrawal: results from a twelve-m. Arthritis Rheum. 2005;52(1):2735.

38. Klarenbeek NB, van der Kooij SM, GülerYüksel M, van Groenendael JHLM, Han $\mathrm{KH}$, Kerstens PJSM, et al. Discontinuing treatment in patients with rheumatoid arthritis in sustained clinical remission: exploratory analyses from the BeSt study. Ann Rheum Dis. 2011;70(2):315-9.

39. Tanaka $Y$, Takeuchi T, Mimori T, Saito K, Nawata M, Kameda H, et al. Discontinuation of infliximab after attaining low disease activity in patients with rheumatoid arthritis: RRR (remission induction by Remicade in RA) study. Ann Rheum Dis. 2010;69(7):128691.

40. van der Maas A, Kievit W, van den Bemt BJF, van den Hoogen FHJ, van Riel PL, den Broeder AA. Down-titration and discontinuation of infliximab in rheumatoid arthritis patients with stable low disease activity and stable treatment: an observational cohort study. Ann Rheum Dis. 2012;71(11):1849-54.

41. Smolen JS, Emery P, Ferraccioli GF, Samborski W, Berenbaum F, Davies OR, et al. Certolizumab pegol in rheumatoid arthritis patients with low to moderate activity: the CERTAIN double-blind, randomised, placebo-controlled trial. Ann Rheum Dis. 2015;74(5):843-50.

42. Saleem B, Keen H, Goeb V, Parmar R, Nizam S, Hensor EMA, et al. Patients with $\mathrm{RA}$ in remission on TNF blockers: when and in whom can TNF blocker therapy be stopped? Ann Rheum Dis. 2010;69(9):163642.

43. Brocq O, Millasseau E, Albert C, Grisot C, Flory $\mathrm{P}$, Roux $\mathrm{C}-\mathrm{H}$, et al. Effect of discontinuing TNFalpha antagonist therapy in patients with remission of rheumatoid arthritis. Jt Bone Spine. 2009;76(4):350-5.

44. Kavanaugh A, Lee SJ, Curtis JR, Greenberg JD, Kremer JM, Soto L, et al. Discontinuation of tumour necrosis factor inhibitors in patients with rheumatoid arthritis in lowdisease activity: persistent benefits. Data from the Corrona registry. Ann Rheum Dis. 
2015;7(4):1150-5.

45. Botsios C, Furlan A, Ostuni R, Sfriso R, Todesco S, Punzi L. Effects of low-dose etanercept in maintaining DAS-remission previously achieved with standard-dose in patients with rheumatoid arthritis. Ann Rheum Dis. 2007;66:54-54.

46. Emery $\mathrm{P}$, Hammoudeh $\mathrm{M}$, FitzGerald $\mathrm{O}$, Combe B, Martin Mola E, Bukowski J, et al. Assessing maintenance of remission with reduced dose etanercept plus methotrexate, methotrexate alone, or placebo in patients with early rheumatoid arthritis who achieved remission with etanercept and methotrextate: the prize study. Ann Rheum Dis. 2014;72(Suppl 3):A399-A399.

47. van Herwaarden N, van der Maas A, Minten MJM, van den Hoogen FHJ, Kievit W, van Vollenhoven RF, et al. Disease activity guided dose reduction and withdrawal of adalimumab or etanercept compared with usual care in rheumatoid arthritis: open label, randomised controlled, noninferiority trial. BMJ. 2015;350:h1389.

48. Haschka J, Englbrecht M, Hueber AJ, Manger B, Kleyer A, Reiser $M$, et al. Relapse rates in patients with rheumatoid arthritis in stable remission tapering or stopping antirheumatic therapy: interim results from the prospective randomised controlled RETRO study. Ann Rheum Dis. 2016;75(1):45-51.

49. van den Broek M, Klarenbeek NB, Dirven L, van Schaardenburg D, Hulsmans HMJ, Kerstens PJSM, et al. Discontinuation of infliximab and potential predictors of persistent low disease activity in patients with early rheumatoid arthritis and disease activity score-steered therapy: subanalysis of the BeSt study. Ann Rheum Dis. 2011;70(8):1389-94.

50. Tracey D, Klareskog L, Sasso EH, Salfeld JG, Tak PP. Tumor necrosis factor antagonist mechanisms of action: a comprehensive review. Pharmacol Ther. 2008;117(2):24479.

51. Arora T, Padaki R, Liu L, Hamburger AE, Ellison AR, Stevens SR, et al. Differences in binding and effector functions between classes of TNF antagonists. Cytokine. 2009;45(2):124-31.

52. Silva LCR, Ortigosa LCM, Benard G. Anti-TNF-a agents in the treatment of immune-mediated inflammatory diseases: mechanisms of action and pitfalls. Immunotherapy. 2010;2(6):817-33.

53. Marotte H, Cimaz R. Etanercept - TNF receptor and IgG1 Fc fusion protein: is it different from other TNF blockers? Expert Opin Biol Ther. 2014;14(5):569-72.

54. Centola M, Cavet G, Shen Y, Ramanujan S, Knowlton N, Swan KA, et al. Development of a multi-biomarker disease activity test for rheumatoid arthritis. PLoS One. 2013;8(4):e60635.

55. Curtis JR, van der Helm-van Mil AH, Knevel R, Huizinga TW, Haney DJ, Shen Y, et al. Validation of a novel multibiomarker test to assess rheumatoid arthritis disease activity. Arthritis Care Res (Hoboken). 2012;64(12):1794-803. 
56. Eastman PS, Manning WC, Qureshi F, Haney D, Cavet G, Alexander C, et al. Characterization of a multiplex, 12-biomarker test for rheumatoid arthritis. J Pharm Biomed Anal. 2012;70:415-24.

57. Rech J, Hueber AJ, Finzel S, Englbrecht M, Haschka J, Manger B, et al. Prediction of disease relapses by multibiomarker disease activity and autoantibody status in patients with rheumatoid arthritis on tapering DMARD treatment. Ann Rheum Dis. 2015;75(9):1637-44.

58. Sanderson T, Morris M, Calnan M, Richards P, Hewlett S. Patient perspective of measuring treatment efficacy: the rheumatoid arthritis patient priorities for pharmacologic interventions outcomes. Arthritis Care Res (Hoboken). 2010;62(5):647-56.

59. Studenic P, Radner H, Smolen JS, Aletaha D. Discrepancies between patients and physicians in their perceptions of rheumatoid arthritis disease activity. Arthritis Rheum. 2012;64(9):2814-23.

60. Steunebrink LMM, Oude Voshaar MAH, Taal E, Vonkeman HE, Zijlstra TR, van de Laar MAFJ. Determinants of perceived health nonimprovement in early rheumatoid arthritis patients with favorable treatment outcomes. Arthritis Care Res (Hoboken). 2018;70(4):510-5.

61. Smolen JS, Breedveld FC, Burmester GR, Bykerk V, Dougados M, Emery $P$, et al. Treating rheumatoid arthritis to target: 2014 update of the recommendations of an international task force. Ann Rheum Dis. 2016;75(1):3-15.
62. Singh JA, Saag KG, Bridges SL, AkI EA, Bannuru RR, Sullivan MC, et al. 2015 American College of Rheumatology Guideline for the Treatment of Rheumatoid Arthritis. Arthritis Rheumatol (Hoboken, NJ). 2016;68(1):1-26.

63. Aletaha D, Snedecor S, Ektare V, Xue M, Bao Y, Garg V. Clinical and economic analysis of outcomes of dose tapering or withdrawal of tumor necrosis factor-a inhibitors upon achieving stable disease activity in rheumatoid arthritis patients. Clin Outcomes Res. 2017;9:451-8. 



\section{Chapter}

\section{Stopping Tumor Necrosis Factor-inhibitors in Patients with Established Rheumatoid Arthritis in Remission or Stable Low Disease Activity: A Pragmatic Randomized Multicenter Open-Label Controlled Trial.}

Harald E. Vonkeman,

Janneke Tekstra,

Dirkjan van Schaardenburg,

M. Starmans-Kool,

E. Brouwer,

Reinhard Bos,

Willem F. Lems,

Edgar M. Colin,

Cornelia F. Allaart,
Inger L. Meek,

Robert B.M. Landewe,

Hein J. Bernelot Moens,

Piet L.C.M. van Riel,

Mart A.F.J. van de Laar,

on behalf of the Dutch National POET

Collaboration. 


\section{ABSTRACT}

\section{OBJECTIVE}

TNF-inhibiting biologicals (TNFi) are effective treatments for rheumatoid arthritis (RA). It is unclear if patients in remission or in stable low disease activity need to continue TNFi or can stop this treatment. This study was undertaken to assess whether RA patients in remission or stable low disease activity can effectively and safely stop their TNFi therapy.

\section{METHODS}

Pragmatic multicenter open-label randomized controlled trial. Inclusion criteria: patients diagnosed with RA according to the ACR 1987 criteria, using a TNFi for at least 1 year with stable dose DMARDs over the last 6 months, DAS28 $<3.2$ over the last 6 months. Patients were randomized to either stop or continue their current TNFi in a 2:1 ratio. Flare was defined as DAS28 $\geq 3.2$ with an increase $\geq 0.6$ compared to the previous DAS28.

\section{RESULTS}

531 patients were allocated to the stop-group and 286 to the TNFi continuation-group. At 12 months, more patients in the stop-group (267/531 [50.3\%]) had experienced a flare than in the continuation-group (52/286 [18.2\%]; $p<0.001)$. The hazard ratio for flare after stopping TNFi was 3.41 (95\% Cl: 2.53-4.59). Mean DAS28 scores in the stop-group were significantly higher during the follow-up period compared with the continuation-group $(p<0.001)$. Of the 195 patients that restarted TNFi after a flare within 26 weeks, 165 (84.6\%) had regained DAS28 $<3.2$ six months later and median time to regained DAS28<3.2 was 12 weeks (95\% Cl: 10.8-13.2).

\section{CONCLUSION}

Stopping TNFi treatment in RA patients in remission or stable low disease activity results in substantially more flares than continuing. 
Modern pharmacotherapy in rheumatoid arthritis (RA) is characterized by early intensive therapy and treatment to the target of remission. Guidelines propagate starting patients on Disease Modifying Anti-Rheumatic Drugs (DMARDs) as soon as possible to achieve clinical remission. When targets are not met treatment should be intensified by increasing or combining conventional synthetic (Cs)DMARDs or by adding biologics such as tumor necrosis factor inhibitors (TNFi) ${ }^{(1-5)}$. However, current guidelines do not provide clearre commendations on treatment strategies after remission or stable low disease activity has been reached ${ }^{(4,5)}$.

TNFi are known to increase the risk of infections and possibly some forms of cancer ${ }^{(6-8)}$. They are also expensive as compared to treatment with conventional synthetic DMARDs. While there have been many studies demonstrating the efficacy of adding TNFi to csDMARDs in attaining disease remission ${ }^{(9-11)}$, few randomized studies into subsequent stopping or tapering TNFi are available. Several small observational studies have suggested that 25$60 \%$ of RA patients on a combination of methotrexate and TNFi may retain low disease activity after stopping their $\mathrm{TNFi}^{(12-17)}$. Some studies also suggest that in the majority of these patients TNFi can be restarted with similar efficacy ${ }^{(12,18)}$.

It is unclear if patients should really continue TNFi to sustain remission or low disease activity or if this treatment can be stopped. Likewise, it is unclear if TNFi can be effectively and safely restarted if necessary. Therefore, patients without notable complications or side effects are often kept on TNFi indefinitely. Because of the potentially avoidable risks and expenses of long-term TNFi treatment, we undertook a nation-wide pragmatic randomized multicenter open-label controlled trial to examine whether RA patients with remission or stable low disease activity can safely and effectively stop TNFi. 


\section{PATIENTS AND METHODS}

\section{SETTING AND PATIENTS}

This pragmatic randomized open-label controlled trial was conducted at 47 rheumatology centers throughout the Netherlands. Written informed consent was obtained from all study patients. Eligibility criteria included: age $>18$ years, RA diagnosis according to the American College of Rheumatology 1987 criteria ${ }^{(19)}$, TNFi treatment for at least one year with stable concomitant csDMARDs use for at least six months prior to inclusion. Patients were in remission or had stable low disease activity for at least six months, defined as either a Disease Activity Score in 28-joints (DAS28) (20) $_{3}$.2 measured in at least two consecutive visits, or the rheumatologists' clinical impression of remission or stable low disease activity in combination with at least one C-reactive protein (CRP) level $<10 \mathrm{mg} / \mathrm{L}$ in the six months prior to inclusion. There were no exclusion criteria. Study inclusion took place from March 2012 to March 2014.

The study was approved by the Ethical Review Boards of all participating hospitals. The study was conducted in accordance with Good Clinical Practice guidelines and the Declaration of Helsinki. The study (POET study) is registered in the Netherlands Trial Register, number NTR3112.

\section{INTERVENTION}

Patients were randomized 2:1 to either stop or continue their TNFi. Computer block randomization was used to achieve balance in allocation per center. All other medications, including cSDMARDs, glucocorticoids and non-steroidal anti-inflammatory drugs (NSAIDs), were left at the discretion of the treating rheumatologists and were continued unchanged as much as possible. In case of flare, defined as a DAS28 $\geq 3.2$ plus an increase $\geq 0.6$ compared to the previous measurement, TNFi treatment could be restarted in the stopgroup or switched in the continuation-group.

\section{OUTCOMES AND FOLLOW-UP}

\section{Baseline Measurements}

Baseline characteristics included: age, sex, weight, length, disease duration, medication use, rheumatoid factor (RF) and anti-cyclic citrullinated peptide (anti-CCP) antibody status.

\section{Efficacy Assessments}

Patients were evaluated by the treating rheumatologist and rheumatology nurse, at baseline and at least once every three months thereafter, or more often if needed, for a period of one year, in accordance with current Dutch guidelines for the diagnosis and 
treatment of RA ${ }^{(3)}$.Clinical measurements, which are part of standard rheumatology care, were performed at every visit and included a tender joint count in 28 joints (TJC28), a swollen joint count in 28 joints (SJC28), erythrocyte sedimentation rate (ESR), and a patient-reported assessment of general health on a $100 \mathrm{~mm}$ visual analog scale (VAS$\mathrm{GH})$. Together, these components were combined into the composite Disease Activity Score for 28 joints (DAS28) ${ }^{(12)}$. DAS28 scores range from 0 to approximately 10 with scores $\leq 3.2$, between 3.2 and 5.1, and >5.1 indicating low, moderate and high disease activity, respectively ${ }^{(21)}$. A score $<2.6$ corresponds to clinicalremission ${ }^{(22)}$. Patients were encouraged to immediately report any adverse events or disease flares to their treating rheumatologist. Physician-reported flares and changes in medication were recorded at each scheduled or unscheduled visit. All data were collected and stored using a tailor-made web based data management system.

\section{Safety Assessments}

Patients were closely monitored for adverse events. Clinical and laboratory results were assessed at each three-monthly visit. Adverse events were recorded at every visit.

\section{Clinical End Point}

The primary end point of the study was the proportion of patients with a flare during 12 -months follow-up. Flare was defined as DAS28 $\geq 3$. 2 plus an increase of $\geq 0.6$ compared to the last available DAS28 ${ }^{(23)}$. Secondary endpoints were time-to-flare, change from baseline in DAS28 score, the number of patients -and time-to- regaining remission (DAS28 $<2.6$ ) or low disease activity (DAS28 <3.2) after restarting TNFi (only in the stop-group), and the proportion of patients with (serious) adverse events.

\section{STATISTICAL ANALYSIS}

The projected sample size for the POET study was based on an estimated proportion of $40 \%$ flares in the stop-group ${ }^{(12)}$ and a 2:1 randomization ratio. The formal sample size calculation indicated that 869 patients would be needed to provide $80 \%$ power to detect a difference of at least $10 \%$ between both groups ( $a=0.05)$. To compensate for an estimated $10-15 \%$ dropout, the study protocol conservatively aimed to include 1000 patients (667 in the stop-group and 333 in the continuation-group) within 1 year.

During the enrollment-phase of the trial it became clear that recruitment was at a slower pace than anticipated and that the target sample size could not be achieved. After an extension of the planned inclusion period with one year, a total of 819 patients had been randomized. Because of slowing enrollment during the final months, while observing a lower than anticipated dropout rate, the steering committee decided to stop enrolment by 
the end of March 2014. Although not completely satisfactory, the estimated power of the study to detect $a \geq 10 \%$ difference between both groups remained as high as $77 \%$.

If a DAS28 score could not be calculated because of a missing value for ESR or VAS-GH, this value was imputed by means of the expectation-maximization algorithm using the patient's values of the remaining components of the DAS28. Missing values for all DAS28 assessments were $8.3 \%, 8.9 \%, 10.4 \%$ and $15.3 \%$ at 3, 6, 9 and 12 months visits, respectively. The primary analysis was performed on the basis of intention-to-treat in patients that were correctly included. The proportions of patients in both groups with a flare within 6 and 12 months of follow-up were compared by separate $x^{2}$ tests. Patients who dropped out early without flare were assumed to remain in remission. Additional modified intention to treat analyses were performed using a 'worst-case scenario', in which all correctly included patients without flare but with a missing DAS28 score at 3 or 6 months, or 9 and 12 months, respectively, were counted as flare in the stop-group and non-flare in the continuationgroup.

Time-to-flare was examined using Kaplan-Meier survival analysis. In this analysis, patients who dropped out before 12 months without flare were censored at the time of withdrawal. Between-group difference in survival was tested by the log-rank test. Sensitivity analyses were performed by repeating the survival analyses using 'physician-reported flare' and 'medication escalation' (defined as reinitiating TNFi or starting or increasing any biological or non-biological DMARD (including glucocorticoids)) as dependent variables. Mean DAS28 over time was compared using a linear mixed model with a compound symmetry structure for the covariance matrix and the group*time interaction as fixed factor. In the stop-group, the number of patients regaining remission (DAS28<2.6) or low disease activity (DAS28 <3.2) after restarting TNFi within 26 weeks after stopping and time-toregained remission were examined using Kaplan-Meijer survival analysis. Safety data werereported descriptively. Differences in adverse events were not tested statistically because such testing is poorly defined and misleading for uncommon events. All analyses were performed using SPSS, version 22. 


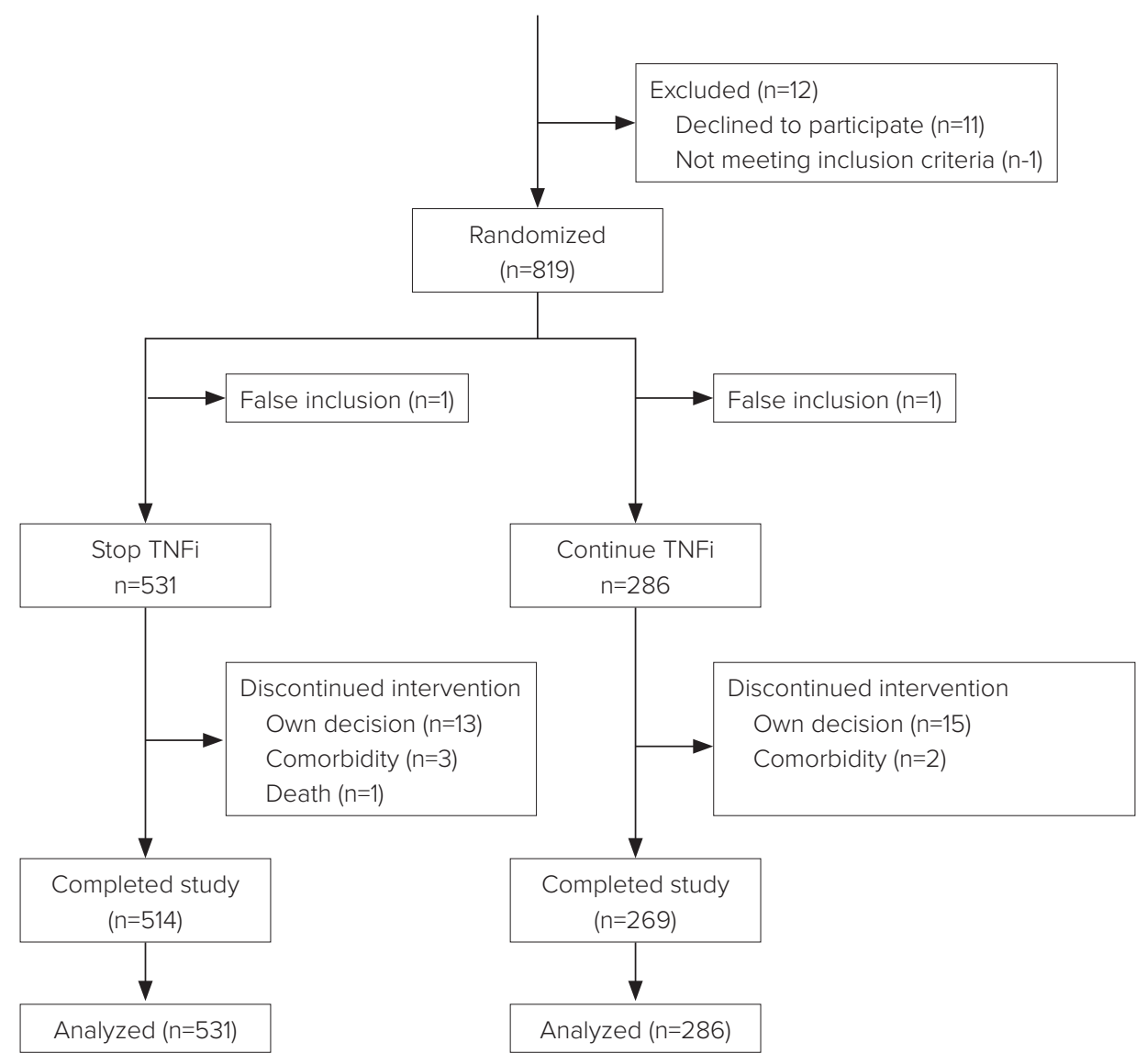

Figure 1. Study flow chart 


\section{RESULTS}

\section{BASELINE CHARACTERISTICS OF PATIENTS}

In total, 817 patients were correctly included, of which 531 were randomized into the TNFi stop-group and 286 to the TNFi continuation-group (Figure 1). Two patients were incorrectly included and excluded immediately after randomization because they did not meet the criteria. Thirty-four patients dropped out during the first 12 months of follow-up on their own decision $(n=28)$, because of comorbidity $(n=5)$ or death $(n=1)$. The proportion of dropouts was slightly higher in the continue-group vs the stop-group (17 out of 531 [3.2\%] vs 17 out of 286 [5.9\%] patients, $p=0.06$ ).

Baseline demographics and disease characteristics were similar in both groups (Table 1). Patients were typically older Dutch caucasian females, with longstanding RF- positive erosive RA. Most were on their first TNFi, primarily adalimumab (49.0\%) or etanercept (42.4\%). Patients had stable low disease activity, in accordance with study inclusion criteria, and 653 patients $(79.9 \%)$ were formally in remission (DAS28<2.6).

Table 1. Baseline Characteristics of Study Patients

\begin{tabular}{lcc}
\hline Characteristic & $\begin{array}{c}\text { Stop TNFi } \\
(\mathbf{n}=53 \mathbf{1})\end{array}$ & $\begin{array}{c}\text { Continue TNFi } \\
(\mathbf{n}=\mathbf{2 8 6})\end{array}$ \\
\hline Female, $\mathrm{n}(\%)$ & $362(68.2 \%)$ & $188(66.0 \%)$ \\
Mean age (SD), $y$ & $60.0(11.8)$ & $59.7(10.6)$ \\
Mean disease duration (SD), $y$ & $12.0(8.8)$ & $11.1(8.4)$ \\
Mean DAS28 (SD) & $1.98(0.76)$ & $2.05(0.73)$ \\
Mean BMI (SD), $\mathrm{kg} / \mathrm{m}^{2}$ & $25.9(4.3)$ & $26.2(4.5)$ \\
RF positive, $\mathrm{n}(\%)$ & $328(67.5 \%)$ & $178(67.4 \%)$ \\
Anti-CCP positive, $\mathrm{n}(\%)$ & $332(68.3 \%)$ & $179(67.8 \%)$ \\
Erosive disease, $\mathrm{n}(\%)$ & $305(62.8 \%)$ & $152(57.6 \%)$ \\
TNFi & & $129(45.1 \%)$ \\
Adalimumab, $\mathrm{n}(\%)$ & $271(51.1 \%)$ & $133(46.5 \%)$ \\
Etanercept, $\mathrm{n}(\%)$ & $213(40.2 \%)$ & $14(4.9 \%)$ \\
Infliximab, $\mathrm{n}(\%)$ & $25(4.7 \%)$ & $8(2.8 \%)$ \\
Golimumab, $\mathrm{n}(\%)$ & $15(2.8 \%)$ & $2(0.7 \%)$ \\
Certolizumab, $\mathrm{n}(\%)$ & $6(1.1 \%)$ & \\
Number of TNFi & & $243(85.0 \%)$ \\
$1^{\text {st }}, \mathrm{n}(\%)$ & $459(86.6 \%)$ & $37(12.9 \%)$ \\
$2^{\text {nd }}, \mathrm{n}(\%)$ & $61(11.5 \%)$ & $6(2.1 \%)$ \\
$3^{\text {rd }}, \mathrm{n}(\%)$ & $10(1.9 \%)$ & \\
\hline
\end{tabular}


Table 1. CONTINUED. Baseline Characteristics of Study Patients

\begin{tabular}{lcc}
\hline Characteristic & $\begin{array}{c}\text { Stop TNFi } \\
(\mathbf{n = 5 3 1 )}\end{array}$ & $\begin{array}{c}\text { Continue TNFi } \\
(\mathbf{n}=\mathbf{2 8 6})\end{array}$ \\
\hline csDMARD & & \\
Methotrexate, $n(\%)$ & $459(86.4 \%)$ & $252(88.1 \%)$ \\
Other DMARD, $n(\%)$ & $43(8.1 \%)$ & $23(8.0 \%)$ \\
No DMARD, $n(\%)$ & $29(5.5 \%)$ & $11(3.8 \%)$ \\
\hline
\end{tabular}

$\mathrm{TNFi}=$ tumor necrosis factor-alpha inhibitor; DAS28 = disease activity score in 28 joints; RF = rheumatoid factor; anti$\mathrm{CCP}=$ anti-cyclic citrullinated peptide; BMI = Body Mass Index; csDMARD = conventional synthetic disease modifying anti-rheumatic drug.

\section{FLARE RATES AND SURVIVAL}

At the time of analysis, follow-up time for all patients was 12 months. Significantly more patients in the stop-group experienced a flare within 6 months (212 out of 531 [39.9\%] vs 36 out of 286 [12.6\%] patients, $p<0.001$ ) or within 12 months (267 out of 531 [50.3\%] vs 52 out of 286 [18.2\%] patients, $p<0.001$ ) compared with the continuation-group. In worst-case scenario analyses, the number of patients with a flare in the stop-group was 258 (48.6\%) and $322(60.6 \%)$ at 6 and 12 months, respectively.

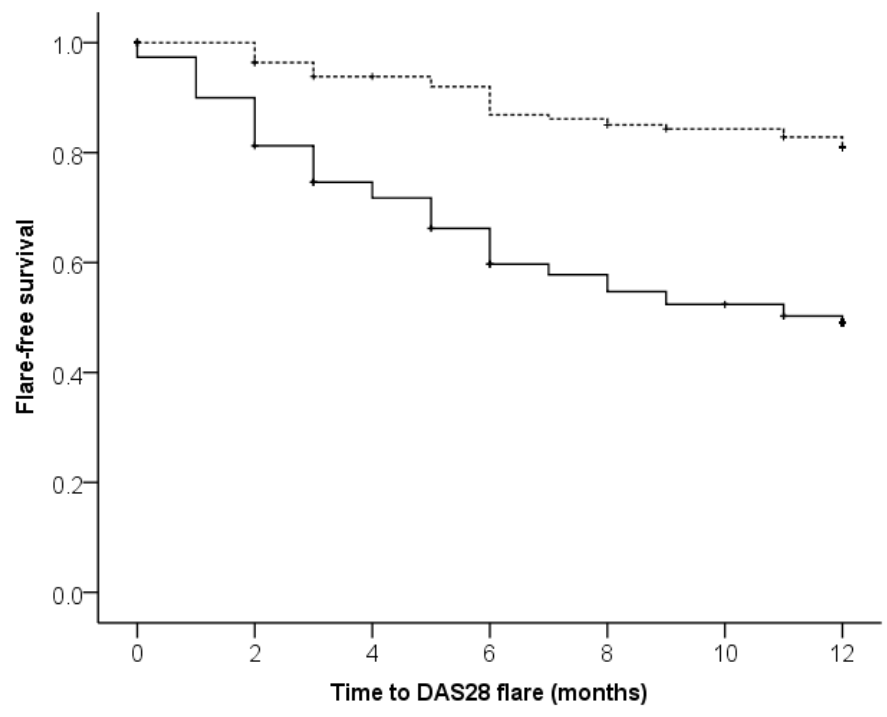

Figure 2. Kaplan-Meier curves for DAS28 flare-free survival. The solid line represents the stopgroup, the dashed line represents the continuation-group. 
Kaplan-Meier analysis confirmed that flare-free survival was significantly $(p<0.001)$ lower in the stop-group than in the continuation-group (Figure 2 ). The hazard ratio for flare after stopping TNFi was 3.4 (95\% Cl: 2.5-4.6). Sensitivity analysis with 'physician-reported flare' and 'medication escalation' as the criteria for flare gave similar results, although 12-month flare rates were somewhat higher for both 'physician-reported flares' (293 out of 531 [55.2\%]) and 'medication escalation' (305 out of 531 [57.4\%]) in the stop-group and clearly lower in the 'continuation-group' (21 out of 531 [7.3\%] and 32 out of 286 [11.2\%], respectively) (Supplementary Figure 1). Flare-free survival was significantly $(p<0.001)$ lower for the stopgroup using both alternative anchors for flare.

\section{DISEASE ACTIVITY OVER TIME}

The mixed effect model showed a significant $(p<0.001)$ interaction between time and group, indicating that mean DAS28 in the stop-group was significantly higher over time compared with the continuation-group (Figure 3). Mean DAS28 was stable over time in the continuation-group. In the stop-group, disease activity scores increased notably at 3 and 6 months and remained stable thereafter but at a higher level than in the continuation-group at 9 and 12 months. The difference in mean DAS28 scores apparently decreased again after 6 months follow-up, probably due to a proportion of patients in the stop group that restarted TNFi. In both groups, mean DAS28 remained below the threshold for moderate disease activity.

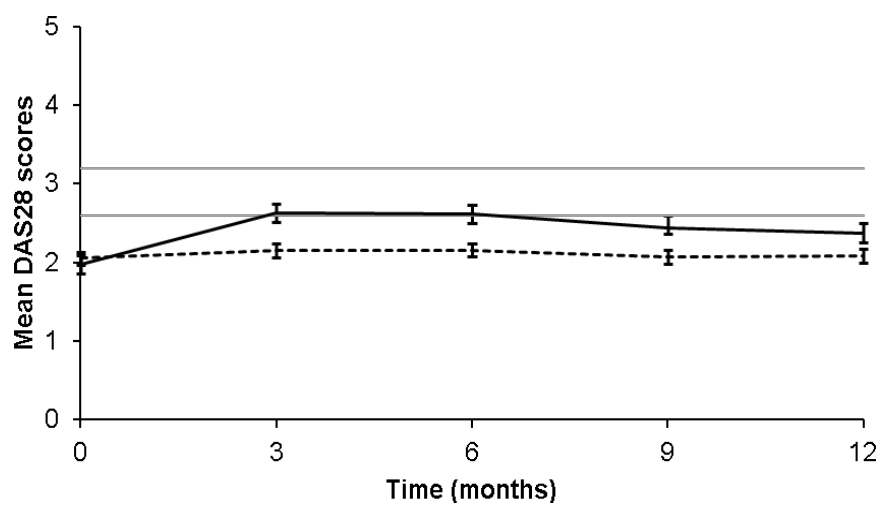

Figure 3. Mean DAS28 scores over time. The solid line represents the stop-group, the dashed line shows the continuation-group. The grey lines represent thresholds for low disease activity (DAS28 $<3.2$ ) and remission (DAS28 <2.6). Error bars represent 95\% confidence intervals. 


\section{REGAINED DISEASE CONTROL}

In total, 252 of 531 patients (47.5\%) in the stop-group restarted TNFi after flare. Of the 195 patients that restarted within 26 weeks after inclusion,132 (67.7\%) achieved clinical remission and an additional 33 (16.9\%) regained low disease activity within 26 weeks. Median time to regained low disease activity or remission upon flare was 12 (95\% Cl: 10.713.3) and 14 weeks (95\% Cl: 11.2-16.8) respectively.

\section{SAFETY}

There were 41 reported serious adverse events (Supplementary Table $\mathbf{1}$ and 2); 1 death (due to an infection in the continuation-group) and 40 hospitalizations (33 [6.2\%] in the stop-group vs 7 [2.4\%] in the continuation-group). Eleven [2.1\%] hospitalizations due to infection occurred in the stop-group vs 4 [1.4\%] in the continuation-group. Hospitalization due to malignancy was reported in 5 patients [0.9\%] in the stop-group vs 3 patients [1.0\%] in the continuation-group. There were also 4 cases of elective surgery in the stop-group: carpal tunnel syndrome, hip osteoarthritis, transurethral resection of the prostate, and fistula excision. Of the 33 hospitalizations in the stop-group, 23 were judged as unrelated and 10 were judged as possibly related to (stopping) TNFi. In the continuation-group, 2 of 7 hospitalizations were judged unrelated to, and 5 possibly related to continuing TNFi. Additionally, there were 143 adverse events (95 [17.9\%] in the stop-group vs 48 [16.8\%] in the continuation-group). Among the patients in the stop-group that restarted TNFi, no allergic reactions were reported.

\section{DISCUSSION}

In this study we demonstrate that stopping TNFi in RA patients in remission or stable low disease activity results in significantly more flares than continuation of TNFi. Patients who stopped TNFi had a more than threefold increased risk of experiencing a flare within 12 months of follow-up as compared to those who continued. Mean disease activity in the stop-group was significantly increased throughout the follow-up period compared with the continuation-group, although the vast majority of patients remained well below the threshold for moderate disease activity. After restarting TNFi treatment most patients in the stop-group quickly regained low disease activity or remission. There were no notable safety issues associated with stopping and restarting TNFi.

The finding that stopping TNFi treatment resulted in more flares is robust, both statistically and clinically and because sensitivity analyses using other definitions of flare yielded similar results. Previous studies of stopping TNFi have shown more divergent results, 
possibly due to heterogeneity instudy designs, definitions of flare and thresholds for disease activity before inclusion. Also, the use of concomitant csDMARDs was not clearly reported in most of these studies.

Recent results from the US Corrona registry suggested that $73.4 \%$ of 717 patients maintained benefit for more than 12 months after stopping their first TNFi ${ }^{(24)}$. All other previous studies examined stopping specific TNFi. The results from an extension of the HONOR study, an open-label non-randomized trial in Japan, showed that $48 \%$ of 75 RA patients maintained remission and 62\% maintained low disease activity for at least 12 months after stopping adalimumab (18). In the smaller retrospective BRIGHT study, however, only $18 \%$ of 22 patients who discontinued their adalimumab monotherapy maintained low disease activity after 12 months ${ }^{(25)}$. In the observational RRR study, again from Japan, 55\% of 102 patients who stopped infliximab maintained low disease activity at 12 months follow-up (13). Finally, a posthoc analysis of the BeSt study from the Netherlands showed that $80 \%$ of 104 patients who stopped infliximab maintained low disease activity for at least 12 months ${ }^{(16)}$. The high rate of successful discontinuation of infliximab in the BeStstudy may be explained by the very early initial treatment with the TNFi infliximab, whereas in the RRR study infliximab was only started after failure of multiple csDMARDs ${ }^{(16)}$.

To date, only two randomized controlled trials of stopping TNFi have been published. Both studies addressed stopping the TNFi etanercept. In the PRESERVE study, a randomized placebo-controlled trial in 834 patients (80 centers world-wide), 604 patients with sustained low disease activity were randomized to stopping or continuing etanercept ${ }^{(26)}$. Results of the PRESERVE study were very similar to the current trial, with $42.6 \%$ of the patients in the stop-group vs $82.6 \%$ in the continuation-group maintaining low disease activity at 12 months follow-up. In the DOSERA study, a European randomized placebo-controlled trial of 73 participants with low disease activity prior to discontinuation of etanercept, only $13 \%$ patients had flare-free survival after 48 weeks ${ }^{(27)}$. In this study, however, different criteria were used to identify possible flare, including patient reported flare.

The current study only examined stopping TNFi completely. Several previous studies (additionally) examined the effects of TNFi dose reduction. Four randomized controlled trials compared etanercept reduction versus stopping or continuation ${ }^{(26-29)}$. Although reduced dosing generally resulted in an increased flare risk, outcomes were better than with stopping. Recently, van Herwaarden et al. ${ }^{(30)}$ showed that disease activity-guided dose reduction of adalimumab and etanercept was non-inferior to dose maintenance with respect to the occurrence of major flares, defined as DAS28 flares with a duration longer than 3 months. However, the incidence of DAS28 flares of shorter duration was significantly 
higher in the dose reduction-group than the continuation-group, with proportions similar to those found in the current study.

Survival analysis showed that $83.1 \%$ of the patients regained low disease activity quickly after restarting TNFi, with a median time to regained low disease activity of 12 weeks. This corresponds well with previous studies that examined this endpoint. In the HONOR study, restarting adalimumab was effective in regaining low disease activity in $90 \%$ of patients within 6 months and in $100 \%$ of patients after 9 months ${ }^{(18)}$. In the BeSt study, $84 \%$ regained low disease activity after restarting infliximab within a median of 3 months ${ }^{(16)}$. In the RRR study, re-treatment with infliximab also resulted in regained low disease activity in the majority of patients within 6 months ${ }^{(12)}$. As the current study was limited to 12 months followup, it was not possible to assess if and when the remaining $16.9 \%$ of patients regained low disease activity.

In the current study, $57.4 \%$ of the patients needed a medication escalation after stopping their TNFi, usually starting or increasing csDMARDs, compared with $11.2 \%$ of the patients in the continuation-group. Only the BRIGHT study also reported on this outcome, showing no significant differences between the patients who stopped or continued adalimumab ${ }^{(26)}$. There was no significant difference in drop-out rate between the stop- and continuation groups, although the rate was numerically higher in the stop-group. There were more hospitalizations in the stop-group than in the continuation-group (6.4\% vs $2.4 \%$ ). Most hospitalizations in the stop-group were due to infections, elective surgery or surgery because of malignancies or fractures. The PRESERVE study likewise reported no statistically significant difference in the total number of adverse events between the etanercept stop- and continuation-group ${ }^{(26)}$. Additionally, there were no notable (serious) adverse events after restarting TNFi.One major concern in stopping and restarting infliximab is the possibility of augmented infusion reactions due to antibody development between administrations. In the RRR and BeSt studies minimal infusion reactions were seen after restarting infliximab in $4.9 \%$ and $10 \%$ of patients, respectively ${ }^{(12,16)}$.

Our study has several strengths. It is the largest pragmatic randomized controlled trial on stopping TNFi in RA patients in remission or stable low disease activity to date. This non-industry-funded trial is the product of nation-wide consensus among investigators in the Netherlands. Most patients had long disease duration (established RA) and an average age of 60 years, which is representative of the TNFi using RA population in the Netherlands. We used strictly protocolled electronic data collection, including safety monitoring. Additionally, we used a strict, discriminatory and valid criterion for flare based on a combination of a threshold and a change over time in DAS28, whereas most other 
studies focused on achieving an absolute DAS28 cut-off only. The latter may be more sensitive but may lack specificity ${ }^{(23)}$.

The study has some limitations. It is an open-label study, which may have influenced patients and rheumatologists in their interpretation of disease activity and their decisions to change medication. Secondly, the study had a standard follow-up of 12 months, which may have been too short to examine the persistence of the effects of stopping TNFi.

In conclusion, stopping TNFi treatment in RA patients in remission or stable low disease activity results in substantially more flares than continuing.

\section{ACKNOWLEDGEMENT}

The authors thank all patients, rheumatology nurses and rheumatologists of participated centers; members of Steering Committee consisting of Renée Allaart, University Medical Center Leiden; Annelies Boonen, Maastricht University Medical Center; Reinhard Bos, Medical Center Leeuwarden; Liesbeth Brouwer, University Medical Center Groningen; Alfons den Broeder, Sint Maartens clinic; Danielle Gerlag, Amsterdam Medical Center; Mieke Hazes, Erasmus University Medical Center; Willem Lems, VUMC; Dirkjan van Schaardenburg, Reade; Janneke Tekstra, University Medical Center Utrecht; Harald Vonkeman, Arthritis Center Twente MST \& University of Twente; and Gerardine Willemsen (patients association); Huib Kooiman, Dutch Ministry of Health, Welfare \& Sports (VWS); Benien Vingerhoeds, Netherlands Organisation for Health Research and Development (ZonMW). 


\section{REFERENCES}

1. Combe B, Landewe R, Lukas C, Bolosiu HD, Breedveld F, Dougados $M$, et al. EULAR recommendations for the management of early arthritis: report of a task force of the European Standing Committee for International Clinical Studies Including Therapeutics (ESCISIT). Ann Rheum Dis. 2007;66(1):34-45.

2. Kiely PDW, Brown AK, Edwards CJ, O'Reilly DT, Ostör AJK, Quinn M, et al. Contemporary treatment principles for early rheumatoid arthritis: a consensus statement. Rheumatology (Oxford). 2009 Jul;48(7):765-72.

3. Schipper LG, Hoekstra M, Vliet Vlieland TPM, Jansen TL, Lems WF, van Riel PLCM. [Practice guideline "Diagnosis and treatment of rheumatoid arthritis"]. Ned Tijdschr Geneeskd. 2009 Jan;153:A944.

4. Singh JA, Furst DE, Bharat A, Curtis JR, Kavanaugh AF, Kremer JM, et al. 2012 update of the 2008 American College of Rheumatology recommendations for the use of disease-modifying antirheumatic drugs and biologic agents in the treatment of rheumatoid arthritis. Arthritis Care Res (Hoboken). 2012 May;64(5):625-39.

5. Smolen JS, Landewé R, Breedveld FC, Buch M, Burmester G, Dougados M, et al. EULAR recommendations for the management of rheumatoid arthritis with synthetic and biological disease-modifying antirheumatic drugs: 2013 update. Ann Rheum Dis. 2014 Mar;73(3):492-509.

6. Dreyer L, Mellemkjær L, Andersen AR, Bennett P, Poulsen UE, Juulsgaard
Ellingsen $\mathrm{T}$, et al. Incidences of overall and site specific cancers in TNFa inhibitor treated patients with rheumatoid arthritis and other arthritides - a follow-up study from the DANBIO Registry. Ann Rheum Dis. 2013 Jan;72(1):79-82.

7. Raaschou P, Simard JF, Holmqvist $M$, Askling J. Rheumatoid arthritis, antitumour necrosis factor therapy, and risk of malignant melanoma: nationwide population based prospective cohort study from Sweden. BMJ. 2013 Jan;346:f1939.

8. Ramiro S, Gaujoux-Viala C, Nam JL, Smolen JS, Buch M, Gossec L, et al. Safety of synthetic and biological DMARDs: a systematic literature review informing the 2013 update of the EULAR recommendations for management of rheumatoid arthritis. Ann Rheum Dis. 2014 Mar;73(3):529-35.

9. Chen Y-F, Jobanputra P, Barton P, Jowett S, Bryan S, Clark W, et al. A systematic review of the effectiveness of adalimumab, etanercept and infliximab for the treatment of rheumatoid arthritis in adults and an economic evaluation of their costeffectiveness. Health Technol Assess. 2006 Nov;10(42):iii - iv, xi - xiii, 1-229.

10. Goekoop-Ruiterman YPM, de VriesBouwstra JK, Allaart CF, van Zeben D, Kerstens PJSM, Hazes JMW, et al. Clinical and radiographic outcomes of four different treatment strategies in patients with early rheumatoid arthritis (the BeSt study): a randomized, controlled trial. Arthritis Rheum. 2005 Nov;52(11):3381-90. 
11. Hochberg MC, Tracy JK, Hawkins-Holt M, Flores $\mathrm{RH}$. Comparison of the efficacy of the tumour necrosis factor alpha blocking agents adalimumab, etanercept, and infliximab when added to methotrexate in patients with active rheumatoid arthritis. Ann Rheum Dis. 2003 Nov;62 Suppl 2:ii136.

12. Tanaka $Y$, Takeuchi T, Mimori T, Saito K, Nawata M, Kameda $\mathrm{H}$, et al. Discontinuation of infliximab after attaining low disease activity in patients with rheumatoid arthritis: RRR (remission induction by Remicade in RA) study. Ann Rheum Dis. 2010 Jul;69(7):1286-91.

13. Roux $\mathrm{CH}$, Brocq O, Breuil V, Albert C, Euller-Ziegler L. Safety of anti-TNFalpha therapy in rheumatoid arthritis and spondylarthropathies with concurrent B or C chronic hepatitis. Rheumatology (Oxford). 2006 Oct;45(10):1294-7.

14. Brocq O, Millasseau E, Albert C, Grisot C, Flory $\mathrm{P}$, Roux $\mathrm{C}-\mathrm{H}$, et al. Effect of discontinuing TNFalpha antagonist therapy in patients with remission of rheumatoid arthritis. Joint Bone Spine. 2009 Jul;76(4):350-5.

15. Saleem B, Keen H, Goeb V, Parmar R, Nizam S, Hensor EMA, et al. Patients with RA in remission on TNF blockers: when and in whom can TNF blocker therapy be stopped? Ann Rheum Dis. 2010 Sep;69(9):1636-42.

16. Van den Broek M, Klarenbeek NB, Dirven L, van Schaardenburg D, Hulsmans HMJ, Kerstens PJSM, et al. Discontinuation of infliximab and potential predictors of persistent low disease activity in patients with early rheumatoid arthritis and disease activity score-steered therapy: subanalysis of the BeSt study. Ann Rheum Dis. 2011 Aug;70(8):1389-94.

17. Van Herwaarden $\mathrm{N}$, den Broeder $\mathrm{AA}$, Jacobs W, van der Maas A, Bijlsma JWJ, van Vollenhoven RF, et al. Down-titration and discontinuation strategies of tumor necrosis factor-blocking agents for rheumatoid arthritis in patients with low disease activity. Cochrane database Syst Rev. 2014 Jan;9:CD010455.

18. Tanaka $\mathrm{Y}$, Hirata S, Kubo S, Fukuyo S, Hanami K, Sawamukai $N$, et al. Discontinuation of adalimumab after achieving remission in patients with established rheumatoid arthritis: 1-year outcome of the HONOR study. Ann Rheum Dis. 2015 Feb;74(2):389-95.

19. Arnett FC, Edworthy SM, Bloch DA, McShane DJ, Fries JF, Cooper NS, et al. The American Rheumatism Association 1987 revised criteria for the classification of rheumatoid arthritis. Arthritis Rheum. 1988;31(3):315-24.

20. Prevoo ML, van 't Hof MA, Kuper $H H$, van Leeuwen MA, van de Putte LB, van Riel PL. Modified Disease Activity Scores that include twenty-eight-joint counts: development and validation in a prospective longitudinal study of patients with rheumatoid arthritis. Arthritis Rheum. 1995;38(1):44-8.

21. Van Gestel AM, Haagsma CJ, van Riel 
PL. Validation of rheumatoid arthritis improvement criteria that include simplified joint counts. Arthritis Rheum. 1998;41(10):1845-50.

22. Fransen J, Creemers MC, Van Riel PL. Remission in rheumatoid arthritis: agreement of the disease activity score (DAS28) with the ARA preliminary remission criteria. Rheumatol. 2004;43(10):1252-5.

23. Van der Maas A, Lie E, Christensen R, Choy E, de Man YA, van Riel P, et al. Construct and criterion validity of several proposed DAS28-based rheumatoid arthritis flare criteria: an OMERACT cohort validation study. Ann Rheum Dis. 2013 Nov;72(11):1800-5.

24. Kavanaugh A, Lee SJ, Curtis JR, Greenberg JD, Kremer JM, Soto L, et al. Discontinuation of tumour necrosis factor inhibitors in patients with rheumatoid arthritis in lowdisease activity: persistent benefits. Data from the Corrona registry. Ann Rheum Dis. 2014 Dec 3;0:1-6.

25. Harigai M, Takeuchi T, Tanaka Y, Matsubara T, Yamanaka H, Miyasaka N. Discontinuation of adalimumab treatment in rheumatoid arthritis patients after achieving low disease activity. Mod Rheumatol. 2012 Nov;22(6):814-22.

26. Smolen JS, Nash P, Durez $P$, Hall $S$, llivanova $E$, Irazoque-Palazuelos $F$, et al. Maintenance, reduction, or withdrawal of etanercept after treatment with etanercept and methotrexate in patients with moderate rheumatoid arthritis (PRESERVE): a randomised controlled trial. Lancet. 2013
Mar 16;381(9870):918-29.

27. Van Vollenhoven RF, Ostergaard $M$, Leirisalo-Repo $M$, Uhlig $T$, Jansson $M$, Larsson E, et al. Full dose, reduced dose or discontinuation of etanercept in rheumatoid arthritis. Ann Rheum Dis. 2015 Apr 14;

28. Botsios C, Furlan A, Ostuni R, Sfriso R, Todesco S, Punzi L. Effects of low-dose etanercept in maintaining DAS-remission previously achieved with standard-dose in patients with rheumatoid arthritis. Ann Rheum Dis. 2007 Jul;66:54-54.

29. Emery $\mathrm{P}$, Hammoudeh $\mathrm{M}$, FitzGerald $\mathrm{O}$, Combe B, Martin Mola E, Bukowski J, et al. Assessing maintenance of remission with reduced dose etanercept plus methotrexate, methotrexate alone, or placebo in patients with early rheumatoid arthritis who achieved remission with etanercept and methotrextate: the prize study. Ann Rheum Dis. 2014 Jan 23;72(Suppl 3):A399-A399.

30. Van Herwaarden N, van der Maas A, Minten MJM, van den Hoogen FHJ, Kievit W, van Vollenhoven RF, et al. Disease activity guided dose reduction and withdrawal of adalimumab or etanercept compared with usual care in rheumatoid arthritis: open label, randomised controlled, noninferiority trial. BMJ. 2015 Jan;350:h1389. 


\section{SUPPLEMENTARY FILES}
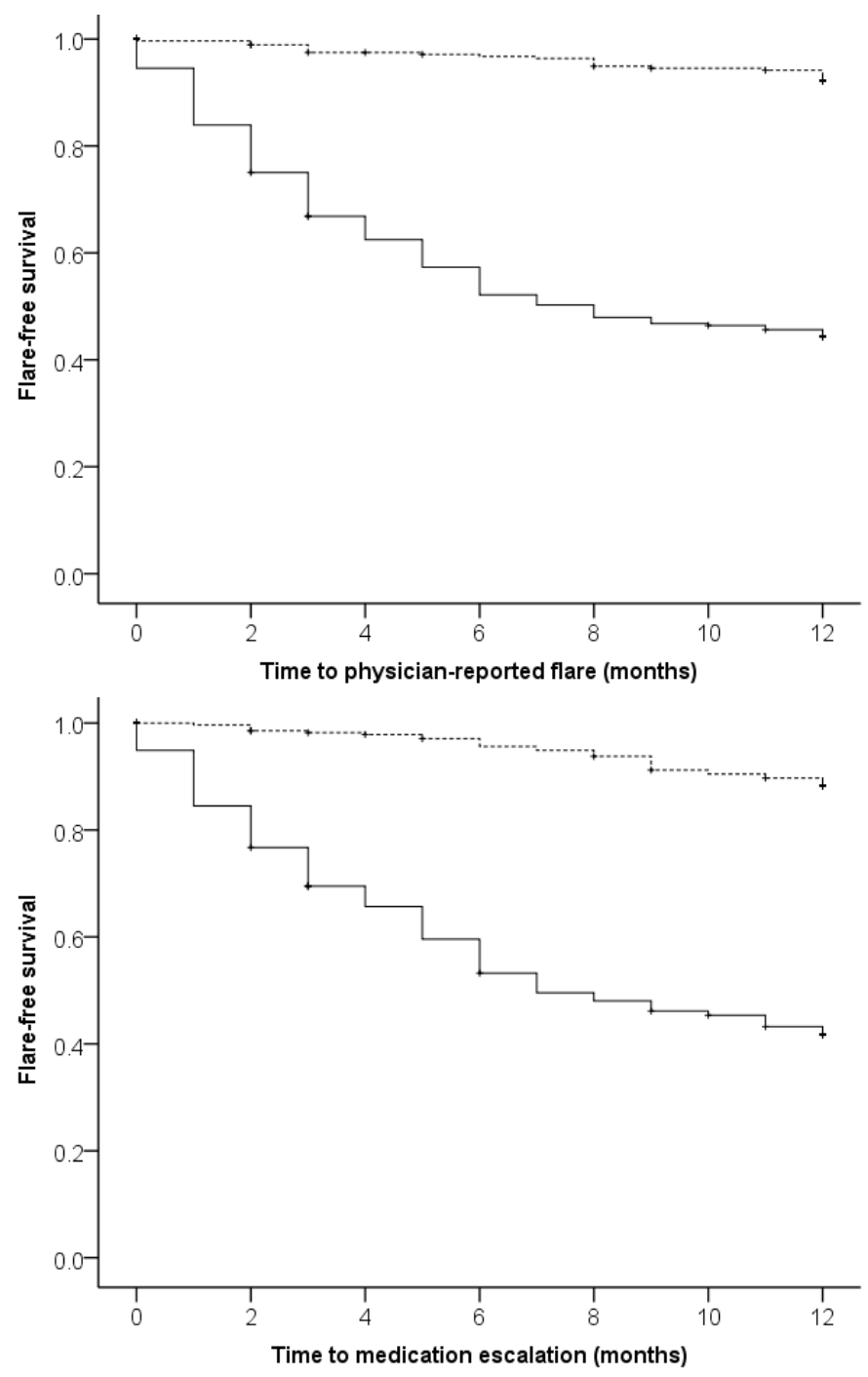

Supplementary Figure 1. Kaplan-Meier curves for flare-free survival based on physician-reported flare (top panel) and medication escalation (bottom panel). The solid line represents the stopgroup, the dashed line shows the continuation-group. 
Supplementary Table 1. Serious adverse events

\begin{tabular}{lcc}
\hline Serious Adverse Events, $\mathbf{n}(\%)$ & Stop TNFi & Continue TNFi \\
\hline Death & 0 & $1(0.3 \%)$ \\
Hospitalization & $34(6.4 \%)$ & $7(2.4 \%)$ \\
Infection & $11(2.0 \%)$ & $4(1.4 \%)$ \\
Malignancy & $5(0.9 \%)$ & $3(1.0 \%)$ \\
Cardiac event & $2(0.4 \%)$ & 0 \\
Cerebral hemorrhage & $2(0.4 \%)$ & 0 \\
Fracture & $4(0.8 \%)$ & 0 \\
Rectal bleeding & $1(0.2 \%)$ & 0 \\
Nephrotic syndrome & $1(0.2 \%)$ & 0 \\
Dyspnea & $1(0.2 \%)$ & 0 \\
Hyperglycaemia & $1(0.2 \%)$ & 0 \\
Vasovagal collaps & $1(0.2 \%)$ & 0 \\
General discomfort & $1(0.2 \%)$ & 0 \\
CTS surgery & $1(0.2 \%)$ & 0 \\
Surgery hip osteoarthritis & $1(0.2 \%)$ & 0 \\
TUR-prostate & $1(0.2 \%)$ & 0 \\
Excision fistula & $1(0.2 \%)$ & 0 \\
\hline
\end{tabular}

Supplementary Table 2. Adverse events

\begin{tabular}{lcc}
\hline Adverse Events, $\mathbf{n}(\%)$ & Stop TNFi & Continue TNFi \\
\hline & & \\
Infection & $40(7.5 \%)$ & $28(9.8 \%)$ \\
Gastro-intestinal complaint & $12(2.6 \%)$ & $1(0.3 \%)$ \\
Musculo-skeletal & $7(1.3 \%)$ & $3(1.0 \%)$ \\
Cardio-pulmonary event & $6(1.1 \%)$ & $3(1.0 \%)$ \\
Fracture & $5(0.9 \%$ & $1(0.3 \%)$ \\
Elevated transaminase & $2(0.4 \%)$ & $1(0.3 \%)$ \\
Hematological & $2(0.4 \%)$ & $1(0.3 \%)$ \\
Neurological & $2(0.4 \%)$ & $1(0.3 \%)$ \\
Malignancy & $1(0.2 \%)$ & $1(0.3 \%)$ \\
Other complaints & $18(3.4 \%)$ & $8(2.8 \%)$ \\
\hline
\end{tabular}





\section{Chapter}

\section{Predictors of Biologic-Free Disease Control in Patients with Rheumatoid Arthritis after stopping Tumor Necrosis Factor Inhibitor Treatment}

Marjan Ghiti Moghadam,

Femke B.G. Lamers-Karnebeek,

Harald E. Vonkeman,

Peter M. ten Klooster,

Janneke Tekstra,

Barbara van Schaeybroeck,

Ruth Klaasen,

Marieke van Onna,

Hein J. Bernelot Moens,

Henk Visser,
Annemarie M. Schilder,

Marc R. Kok,

Robert B.M. Landewé,

Piet L.C.M. van Riel,

Mart A.F.J. van de Laar,

Tim L. Jansen,

on behalf of the Dutch National POET

Collaboration. 


\section{ABSTRACT}

\section{BACKGROUND}

The aim of this study was to identify predictors of prolonged disease control after discontinuation of tumor necrosis factor inhibitor (TNFi) treatment in patients with rheumatoid arthritis (RA).

\section{METHODS}

Post-hoc analysis of 439 RA patients with longstanding RA in remission or with stable Iow disease activity, randomized to stopping TNFi treatment in the multicenter POET trial. Prolonged acceptable disease control was defined as not restarting TNFi treatment within 12 months after stopping. Baseline demographic and disease-related variables were included in univariate and multivariate logistic regression analysis for identifying predictors of relapse.

\section{RESULTS}

One year after baseline, 220 patients (50.1\%) had not restarted TNFi treatment. Antibody type TNFi (OR = 2.36), $\leq 10$ yrs. disease duration $(\mathrm{OR}=2.16)$ and low or moderate multibiomarker disease activity (MBDA) scores $(O R=2.27)$ were independently predictive of successful TNFi discontinuation. Results were similar when using no physician-reported flare as the criterion. TNFi-free survival was significantly different for patient groups based on the number of predictors present, ranging from $21.4 \%$ of patients with no predictor present to $66.7 \%$ of patients with all three predictors present.

\section{CONCLUSION}

Patients using an antibody type TNFi, with shorter disease duration and low or moderate baseline MBDA score are most likely to achieve prolonged disease control after TNFi discontinuation.

\section{TRIAL REGISTRATION}

Netherlands Trial Register NTR3112, 21 October 2011.

\section{KEYWORDS}

Rheumatoid arthritis, Remission, Low disease activity, TNFi discontinuation, predictors. 
Rheumatoid arthritis (RA) is a chronic inflammatory disease which may cause progressive joint damage and increasing physical disability (1). Early and effective treatment has markedly improved outcomes in RA patients ${ }^{(2)}$. The use of conventional synthetic disease modifying anti-rheumatic drug (cSDMARDs) in combination with the more expensive biological DMARDs (bDMARDs) such as tumor necrosis factor inhibitors (TNFi) is effective for reaching remission or low disease activity (LDA) ${ }^{(3-5)}$. Once stable LDA or remission has been reached, patients often continue their combination therapy indefinitely. This practice may however lead to significant overtreatment and lack of efficiency, as recent studies suggest that many RA patients in sustained remission or LDA can taper or altogether stop their TNFi without incurring disease flare ${ }^{(6,7)}$.

Although current guidelines suggest that tapering or withdrawal of bDMARDs can be considered for patients in persistent remission ${ }^{(8)}$, validated predictors of successful discontinuation are needed to successfully implement practical stopping rules in daily clinical practice ${ }^{(9)}$. Several studies have already explored predictors of successful TNFi discontinuation, but it remains a challenge to judge which patients can effectively and safely stop their TNFi without incurring disease flares ${ }^{(10)}$. A recent systematic review found no consistently strong predictors for successful dose reduction or discontinuation ${ }^{(11)}$. Only shorter disease duration ${ }^{(12-14)}$, less or no erosive disease ${ }^{(13-15)}$, and lower disease activity (13-16) at baseline have been shown in more than one study to predict successful TNFi discontinuation. Additionally, single studies have found younger age ${ }^{(13)}$, normal body mass index (BMI) ${ }^{(15)}$, non-smoking ${ }^{(14)}$, anti-cyclic citrullinated peptide antibodies (ACPA) negativity (16, 17), shorter ${ }^{(14)}$ or longer ${ }^{(18)}$ TNFi treatment, and initial (versus delayed) TNFi treatment (12) were associated with successful TNFi discontinuation. Finally, two studies have found a tendency for more relapses among female patients ${ }^{(12,16)}$.

Moreover, two main types of TNFi with different mechanisms of action are used in RA: anti-TNF monoclonal antibodies and soluble TNF receptors. Monoclonal antibody agents like adalimumab and infliximab bind specifically to TNF, while the receptor fusion protein etanercept, functioning as a decoy receptor, binds to circulating TNF and prevents it from interacting with a cell surface receptor ${ }^{(19-22)}$. Adalimumab and etanercept are currently the most frequently prescribed TNFi and generally demonstrate comparable effectiveness ${ }^{23,}$ 24). Previous discontinuation studies, however, generally examined one specific TNFi agent only or did not examine possible differences in the predictive value of the type of TNFi for successful discontinuation.

Recently, the POET trial showed that, although stopping TNFi treatment in patients with established RA in remission or with stable low disease activity resulted in more flares than 
did continuation of TNFi, around half of the patients could successfully stop for at least 12 months ${ }^{(25)}$. The aim of the current study was to identify predictors of prolonged disease control after discontinuation of TNFi.

\section{METHODS}

\section{PATIENTS AND STUDY DESIGN}

We used date from the Dutch POET trial (Netherlands Trial Register NTR3112) ${ }^{(25)}$. This pragmatic, multicenter, open-label, clinical trial randomized RA patients with stable LDA 2:1 to either stop or continue TNFi treatment. Inclusion ran from March 2012 to March 2014. Written informed consent was obtained from all patients. Patients were $\geq 18$ years old, had RA according to the ACR 1987 criteria, and had received TNFi treatment for at least one year prior to inclusion. Patients had been in stable LDA for at least six months, defined as either two Disease Activity Scores in 28 joints using the erythrocyte sedimentation rate (DAS28-ESR) measurements $<3.2$ or the rheumatologists clinical impression of remission or stable low disease activity with at least one CRP measurement $<10 \mathrm{mg} / \mathrm{L}$ in the six months prior to inclusion. Nearly all patients were receiving concomitant csDMARDs and in the 6 months prior to inclusion, no dosage changes were allowed for csDMARDs or corticosteroids.

In total in POET 531 patients were randomized to stop TNFi treatment and were subsequently followed for 12 months ${ }^{(25)}$. Concomitant treatment with cSDMARDs was continued. If RA flared, defined as a DAS28-ESR $\geq 3.2$ with a change in DAS28-ESR $>0.6^{(26)}$, TNFi could be restarted at the discretion of the treating rheumatologist. Because the current study aims to identify patient and clinical predictors of prolonged disease control after discontinuation of TNFi treatment, only data from 439 patients randomized to the stop group and for whom baseline serum samples were collected to measure the multi-biomarker disease activity $(\mathrm{MBDA})^{(27)}$ score were used. Baseline demographic and clinical data were similar between patients with or without a baseline MBDA sample ${ }^{(28)}$.

\section{MEASUREMENTS}

Patients were evaluated by their treating rheumatologist and rheumatology nurse at baseline and at least once every 3 months thereafter. Baseline measures included: age, sex, weight, height, body mass index (BMI), disease duration, rheumatoid factor (RF) and ACPA status, Multi-Biomarker Disease Activity (MBDA) score, erosive disease (yes/no) and medication use, including type of TNFi (antibody vs. receptor antagonist) and concomitant conventional synthetic DMARD (csDMARD) use. 
Clinical measurements were performed at every scheduled or unscheduled visit and included the ESR (mm/h), CRP (mg/l), 28-joint tender joint count (TJC28), 28-joint swollen joint count (SJC28), and a patient-reported assessment of general health on a $100-\mathrm{mm}$ visual analog scale (VAS-GH). These component measures were combined to calculate the DAS28-ESR ${ }^{(29)}$. Physician-reported flares and all changes in medication were recorded throughout the study.

The MBDA score is a novel test that measures 12 biomarkers in serum to assess disease activity in patients with rheumatoid arthritis ${ }^{(27)}$. The algorithm generates a total score on a scale of 1 to 100, with validated categories for low (<30), moderate (30 to 44) and high $(>44)$ activity ${ }^{(30)}$.

\section{STATISTICAL ANALYSIS}

Successful discontinuation was defined as not restarting TNFi treatment within 12 months after stopping. First, univariate logistic regression analyses were performed to examine associations between potential baseline predictors and successful 12-month TNFi discontinuation. The following patient and clinical variables were considered: type of TNFi (antibody vs. receptor antagonist), concomitant csDMARD use, female sex, younger age ( $\leq 60$ yrs.), shorter disease duration ( $\leq 10$ yrs.), RF negativity, ACPA negativity, non-erosive disease, normal weight $(\mathrm{BMI}=18.5-25)$, first TNFi agent, deep remission (DAS28 $\leq 1.98$ ) (31,32), and low or moderate MBDA score $(\leq 44)$. To facilitate the interpretation of odds ratios (ORs), continuous predictors were dichotomized by median split (DAS28-ESR, age, disease duration) or previously established relevant cut-off points (BMI, MBDA). ORs are classified as weak when about 1.5, moderate when about 2.5, strong when about 4 and very strong when about $10{ }^{(33)}$. Additional separate models were run to test for possible interactions between each predictor and type of TNFi. Predictors univariately associated with outcome $(P<0.10)$ were included in a multivariate logistic regression model to identify independent predictors. The multivariate model was reduced by excluding predictors from the model with $P>0.10$ (backward deletion), and the goodness of fit of the final model was estimated using the Hosmer and Lemeshow test ${ }^{\left({ }^{34}\right)}$, where a non-significant result indicates support for the model. The explained variance of the model was examined using Nagelkerke's pseudo $\mathrm{R}^{2}$. As 38 patients (8.7\%) had missing baseline values on laboratory and radiographic erosion data, multiple imputation with 10 imputed datasets was used and pooled ORs are presented. As a sensitivity analysis, the final multivariate model was repeated using no physician-reported flare within 12 months after stopping as the dependent variable. To further explore the prognostic value of the remaining predictors, TNFi-free survival based on the number of predictors present was examined using KaplanMeier survival analysis. Between-group difference in survival was tested using the log rank 
test. Since MBDA testing is not common in many rheumatology practices, the multivariate and survival analyses were repeated without MBDA as a predictor. All statistical calculations were performed using SPSS 23.

\section{RESULTS}

Table 1 summarizes the baseline characteristics of the patients. Median age and disease duration was 60 and 10 years, respectively. Approximately $60 \%$ of the patients used an antibody agent, most frequently adalimumab, while 40\% used etanercept. Most patients were on their first TNFi. In total, 219 (49.9\%) of the patients restarted their TNFi within 12 months, while at least one physician-reported flare was reported for 251 (57.2\%) patients.

Antibody type TNFi, shorter disease duration, non-erosiveness and low or moderate MBDA were weakly to moderately associated with successful discontinuation, defined as not restarting TNFi treatment within 12 months after stopping in univariate regression analysis (Table 2). No interactions with type of TNFi were significant and separate univariate analyses for both types of TNFi showed that the predictive value of individual variables was similar for patients discontinuing an antibody agent or etanercept. However, MBDA $\leq 44$ was significantly predictive only in patients discontinuing etanercept (OR $=3.69 ; 95 \%$ $\mathrm{Cl}: 1.34-10.18 ; \mathrm{P}=0.012)$ and not in patients discontinuing an antibody agent $(\mathrm{OR}=1.68$; 95\% Cl: $0.81-3.45 ; P=0.162)$.

In multivariate analysis, non-erosiveness lost its significance $(P=0.212)$. Type of TNFi, shorter disease duration and low or moderate MBDA scores remained independently predictive of successful discontinuation, with type of TNFi being the strongest predictor (Table 3). As a sensitivity analysis, using 'no physician-reported flare' as the criterion for successful TNFi discontinuation resulted in similar findings in the total sample, with the same three predictors remaining significant in multivariate analysis. However, the predictive value of the variables tended to be slightly lower with ORs of 1.86 (95\% Cl: $1.24-2.79 ; \mathrm{P}=0.003)$, 1.78 (95\% Cl: 1.18 - 2.70; $\mathrm{P}=0.006)$ and 2.49 (95\% Cl: 1.35 - 4.59; $\mathrm{P}=0.003)$ for antibody $\mathrm{TNFi}$, shorter disease duration and low or moderate MBDA score, respectively. 


\section{Table 1. Baseline characteristics of the patients $(\mathbf{N}=\mathbf{4 3 9})$}

\begin{tabular}{|c|c|}
\hline Female, n (\%) & 296 (67.4\%) \\
\hline Age (yrs.), mean (SD) & $59.8(10.8)$ \\
\hline Disease duration (yrs.), median (IQR) & $10(6-17)$ \\
\hline BMI, mean (SD) & $25.9(4.3)$ \\
\hline Normal BMI, n (\%) & 174 (39.6\%) \\
\hline RF positive, n (\%) & $270(67.3 \%)$ \\
\hline ACPA positive, $n(\%)$ & 277 (69.1\%) \\
\hline Erosive disease, n (\%) & $252(62.8 \%)$ \\
\hline ESR, median (IQR) & $9.0(5-17)$ \\
\hline TJC28, median (IQR) & $0(0-1)$ \\
\hline SJC28, median (IQR) & $0(0-0)$ \\
\hline PGA, median (IQR) & $20.7(9.0-28.1)$ \\
\hline DAS28-ESR & $2.0(0.8)$ \\
\hline MBDA score, mean (SD) & $30.2(12.6)$ \\
\hline Low or moderate, n (\%) & 375 (85.4\%) \\
\hline \multicolumn{2}{|l|}{ Type of TNFi } \\
\hline Etanercept & $176(40.1 \%)$ \\
\hline Adalimumab & 225 (51.3\%) \\
\hline Infliximab & $22(5.0 \%)$ \\
\hline Golimumab & $14(3.2 \%)$ \\
\hline Certolizumab & $2(0.5 \%)$ \\
\hline \multicolumn{2}{|l|}{ Number of TNFi, n (\%) } \\
\hline $1^{\mathrm{st}}$ & $379(86.5 \%)$ \\
\hline $2^{\text {nd }}$ & 50 (11.4\%) \\
\hline $3^{\text {rd }}$ & $9(2.1 \%)$ \\
\hline \multicolumn{2}{|l|}{ csDMARD, n (\%) } \\
\hline Methotrexate & 382 (87.0\%) \\
\hline Other csDMARD & 35 (8.0\%) \\
\hline No DMARD & $22(5.0 \%)$ \\
\hline
\end{tabular}

$\mathrm{TNFi}=$ tumor necrosis factor-alpha inhibitors; DAS28 = disease activity score in 28 joints; BMI = body mass index; $\mathrm{RF}=$ rheumatoid factor; $\mathrm{ACPA}=$ anti-cyclic citrullinated peptide antibodies; $\mathrm{ESR}$ = erythrocyte sedimentation rate; CRP = C-reactive protein; TJC28 = 28-joint tender joint count; SJC28 = 28-joint swollen joint count; PGA = patient global assessment; MBDA = multi-biomarker disease activity; cSDMARD = conventional synthetic disease modifying anti-rheumatic drug. 
Table 2. Univariate associations of baseline variables with successful TNFi discontinuation

\begin{tabular}{|c|c|c|c|}
\hline Predictor & OR & $95 \% \mathrm{Cl}$ & $\mathbf{P}$ \\
\hline Type of TNFi (antibody vs. receptor antagonist) & 2.26 & $1.53-3.34$ & $<0.0001$ \\
\hline Concomitant DMARD & 1.21 & $0.51-2.85$ & 0.670 \\
\hline Female sex & 1.07 & $0.72-1.60$ & 0.735 \\
\hline Younger age ( $\leq 60$ yrs.) & 1.17 & $0.80-1.70$ & 0.417 \\
\hline Shorter disease duration ( $\leq 10$ yrs.) & 2.00 & $1.34-2.98$ & 0.001 \\
\hline RF negative & 1.14 & $0.75-1.74$ & 0.530 \\
\hline ACPA negative & 1.06 & $0.70-1.62$ & 0.775 \\
\hline Non-erosive & 1.62 & $1.08-2.44$ & 0.020 \\
\hline Normal weight (BMI 18.5 - 25) & 1.35 & $0.92-1.98$ & 0.128 \\
\hline First TNFi & 1.23 & $0.71-2.13$ & 0.461 \\
\hline DAS28 deep remission (DAS28 $\leq 1.98$ ) & 1.21 & $0.83-1.77$ & 0.314 \\
\hline Low or moderate MBDA $(\leq 44)$ & 2.32 & $1.32-4.05$ & 0.003 \\
\hline
\end{tabular}

$\mathrm{TNFi}=$ tumor necrosis factor-alpha inhibitors; csDMARD = conventional synthetic disease modifying anti-rheumatic drug; $\mathrm{RF}=$ rheumatoid factor; $\mathrm{ACPA}=$ anti-cyclic citrullinated peptide; $\mathrm{BMI}=$ body mass index; $\mathrm{DAS} 28$ = disease activity score in 28 joints; MBDA = multi-biomarker disease activity.

Table 3. Multivariate associations with successful TNFi discontinuation with and without MBDA score as a predictor

\begin{tabular}{lcccccc}
\hline & \multicolumn{3}{c}{ With MBDA } & \multicolumn{3}{c}{ Without MBDA } \\
Predictor & OR & $\mathbf{9 5 \%} \mathbf{~ C ~}$ & $\mathbf{P}$ & OR & $\mathbf{9 5 \%} \mathbf{~} \mathbf{~}$ & $\mathbf{P}$ \\
\hline Antibody TNFi & 2.36 & $1.57-3.54$ & $<0.0001$ & 2.38 & $1.60-3.56$ & $<0.0001$ \\
Disease duration $<10$ yrs. & 2.16 & $1.42-3.29$ & $<0.0001$ & 2.05 & $1.37-3.06$ & $<0.0001$ \\
MBDA score $\leq 44$ & 2.27 & $1.27-4.04$ & 0.006 & - & - & - \\
\hline
\end{tabular}

TNFi = tumor necrosis factor-alpha inhibitors; MBDA = multi-biomarker disease activity. Odds ratios (ORs) are pooled estimated from 10 imputed datasets. Hosmer and Lemeshow with MBDA X2(5) =1.64-3.41, P = 0.637-0.885, Nagelkerke $R^{2}=0.08-0.10$; without MBDA X2(2) =0.00-0.463, $P=0.793-1.000$, Nagelkerke $R^{2}=0.08-0.10$

TNFi-free survival was significantly different (log rank $=43.9, \mathrm{P}<0.001)$ for patient groups based on the number of predictors present (Figure 1). TNFi-free survival rates were $21.4 \%$ in patients with no predictor present $(n=14), 31.7 \%$ in patients with one predictor $(n=104)$, $52.6 \%$ in patients with two predictors $(n=213)$, and $66.7 \%$ in patients with three predictors $(n=108)$ present. Fairly similar results and differences between groups (log rank $=33.9$, $\mathrm{P}<0.001)$ were obtained in TNFi-free survival when using only antibody type TNFi and shorter disease duration as predictors. In this analysis, TNFi-free survival rates were $32.1 \%$ in patients with no predictor present ( $n=84), 48.5 \%$ in patients with one predictor ( $n=231)$, and $65.3 \%$ in patients with both predictors $(n=108)$ present. 

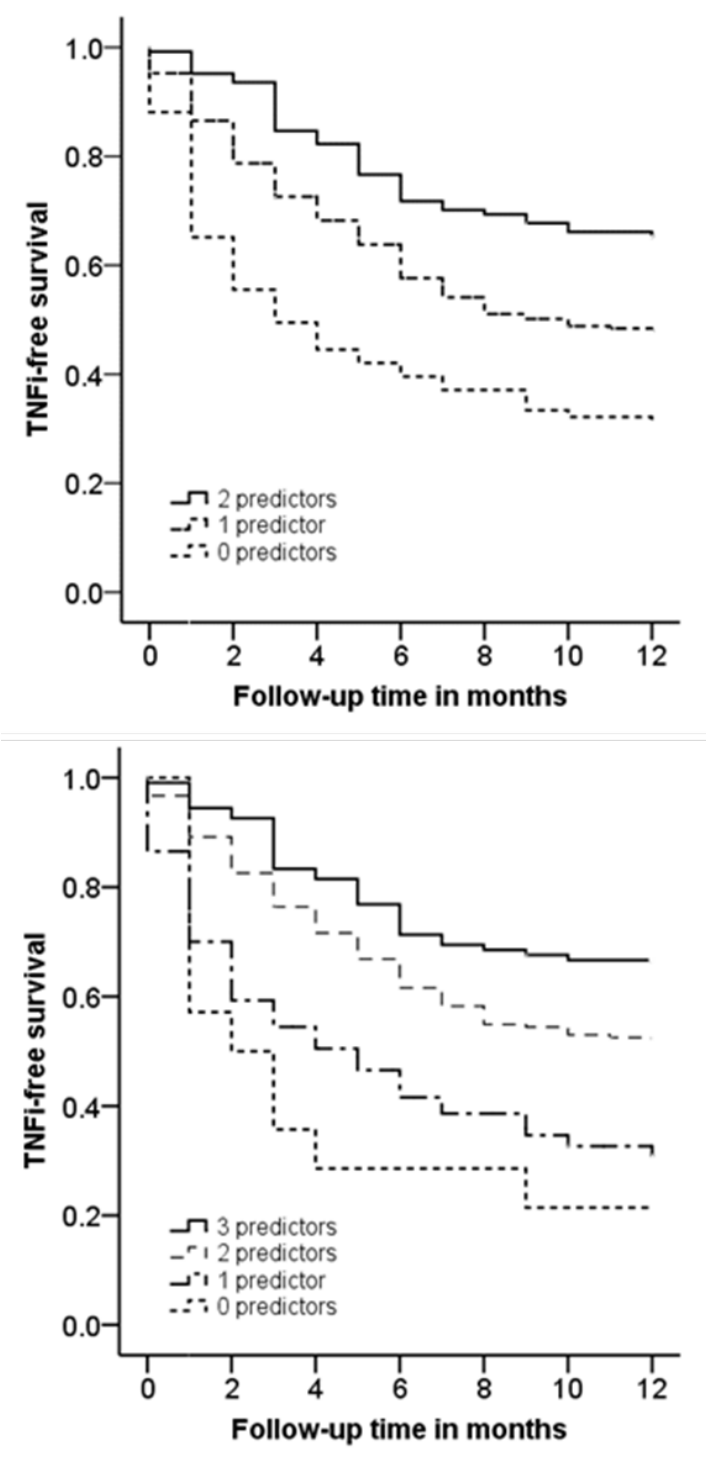

Figure 1. Kaplan-Meier curves showing the proportion of patients not restarting TNFi per number of predictors present.

Upper panel: with MBDA as predictor; Lower panel: without MBDA as predictor. MBDA = multibiomarker disease activity. 


\section{DISCUSSION}

We previously demonstrated that in patients with established RA in sustained remission or with stable low disease activity, as defined by the DAS28-ESR, approximately $50 \%$ of the patients could successfully stop their TNFi for at least 12 months ${ }^{(25)}$. However, with the consequential fifty-fifty chance of relapsing, it would be helpful to identify patient and clinical predictors of prolonged disease control after discontinuation of TNFi. Our analysis of the POET study showed that in RA patients in sustained remission or stable LDA, the type of TNFi (antibody vs. receptor antagonist) being used, RA disease duration and the MBDA score at the time of discontinuation of TNFi were significantly associated with success of stopping. Approximately $70 \%$ of patients who were on a TNFi antibody, with a disease duration of $<10$ years and a MBDA score $\leq 44$ were able to successfully stop their TNFi, while conversely $80 \%$ of patients on a TNFi receptor antagonist, with a disease duration of $\geq 10$ years and a MBDA score $>44$ restarted their TNFi within 12 months of stopping.

Several other studies have previously explored predictors of disease relapse after TNFi discontinuation (10, 11). Several previous studies found shorter disease duration, RF positivity, non-smoking, erosive disease and normal body mass index (BMI) to be associated with successful TNFi discontinuation ${ }^{(13,15)}$. In the current study, shorter disease duration was also a strong predictor of successful discontinuation, but RF positivity and normal BMI were not found to be predictors of successful discontinuation. Erosive disease was univariately associated with restarting TNFi but did not remain a unique predictor in the final multivariate model.

Greater clinical disease activity at the time of discontinuation has previously also been identified as a predictor, although results varied considerably between studies ${ }^{(12,13,35)}$. Our study found that higher baseline DAS28-ESR scores were not associated with the criterion of restarting TNFi treatment within 12 months. However, a high MBDA score at the time of TNFi discontinuation was a predictor of restarting TNFi. Several previous studies also found that MBDA scores may be elevated when conventional clinical measures indicate remission or LDA ${ }^{(17,36-38)}$. Furthermore, these patients were found to be at increased risk for progressive joint damage ${ }^{(36-38)}$. In patients with high baseline MBDA scores at the time of TNFi discontinuation in POET, discontinuation may have allowed a resurgence of subclinical residual inflammation and the subsequent need to restart TNFi treatment ${ }^{(17)}$.

The type of TNFi, i.e. antibody vs. receptor antagonist, has not previously been identified as a predictor of successful discontinuation of TNFi treatment in patients with established RA in remission or with stable low disease activity. Our analysis of the POET study demonstrated 
that patients who were on TNF antibodies (mostly adalimumab) were significantly more often able to successfully discontinue their TNFi than patients who had been using TNF receptor antagonists (mostly etanercept). These findings might possibly be explained by several differences between the TNF receptor antagonists and antibodies. Firstly, TNF receptor antagonists function as decoy receptors that bind to circulating TNF, thereby mimicking the inhibitory effects of naturally occurring soluble TNF receptors, albeit with a greatly extended circulatory half-life. TNF antibodies also bind specifically to TNF and thus block its interaction with the p55 and p75 cell surface TNF receptors. However, TNF antibodies may also lyse surface TNF expressing blood cells in the presence of complement thereby possibly inducing prolonged disease control. Secondly, pharmacokinetic properties differ between drugs, with a single dose half-life of approximately 3 days for the TNF receptor antagonists etanercept vs. 14 days for the TNF antibody adalimumab. Finally, there may be differences in drug dosages and dosage-intervals.

Our study has several strengths. The data were collected in POET, the largest randomized controlled trial on stopping TNFi in RA patients in remission or stable low disease activity to date. Predictors of prolonged disease control after discontinuation of TNFi were analyzed in an unbiased selection of 439 patients in the stop group, comprising 83\% of those who stopped TNFi treatment in POET. Most patients in our study had long disease duration (i.e., established RA) and an average age of 60 years, which is representative of the TNFi-using RA population in The Netherlands. In our analysis, successful discontinuation was defined as not restarting TNFi treatment within 12 months after stopping.

A possible limitation of the study is that the predictors were measured only at baseline. Longitudinal measurements of variables such as DAS28, ESR/CRP or MBDA scores may have provided insight into the effects of TNFi discontinuation over time, and the potential for early changes in these variables to predict relapse. Another possible limitation was the decision to leave restarting of TNFi at the discretion of the treating rheumatologists. It is possible that this may have led to an overestimation of successful disease control and bias in the population of TNFi restarters.

In conclusion, in patients with established RA in sustained remission or stable LDA, the type of TNFi (antibody vs. receptor antagonist) being used, RA disease duration and the MBDA score at the time of discontinuation of TNFi were significantly associated with success of stopping TNFi.

\section{ABBREVIATIONS}

ACPA: anti-cyclic citrullinated peptide antibodies; bDMARD: Biological synthetic disease 
modifying anti-rheumatic drug; BMI: body mass index; CRP: C-reactive protein; CSDMARD: Conventional synthetic disease modifying anti-rheumatic drug; DAS28: disease activity score in 28 joints; DMARD: Disease modifying anti-rheumatic drug; ESR: erythrocyte sedimentation rate; LDA: Iow disease activity; MBDA: multi-biomarker disease activity; OR: odds ratio; PGA: patient global assessment; RA: rheumatoid arthritis; RF: rheumatoid factor; SJC28: 28-joint swollen joint count; TNFi: tumor necrosis factor-alpha inhibitors; TJC28: 28-joint tender joint count.

\section{DECLARATIONS}

Ethics approval and consent to participate

The study was approved by the Ethical Review Boards of all participating hospitals. Ethical approval for the study was granted by the Committee on Research involving Human Subjects, region Arnhem-Nijmegen (Commissie Mensgebonden Onderzoek regio ArnhemNijmegen) and local feasibility by all regional Ethical Committees. All participants provided written informed consent.

Consent for publication

Not applicable.

\section{AVAILABILITY OF DATA AND MATERIALS}

The datasets generated and analyzed during the current study are not publicly available due to legal restrictions related to data privacy protection. However, the data are available upon reasonable request to all interested researchers after authorization of the POET steering committee. Researchers interested in data access may contact the PI of the POET study Tim L. Jansen (tjansen@viecuri.nl) to apply for access.

\section{COMPETING INTERESTS}

The authors declare that they have no competing interests.

\section{FUNDING}

The POET study was funded by the Netherlands Organization for Health Research and Development (ZonMw), project number 152041002. ZonMw had no role in the study design, data collection and analysis, decision to publish, or preparation of the manuscript. Crescendo Bioscience funded the shipping of serum samples to its laboratory and the generation of biomarker data, but did not have any role in the study design, data collection or decision to publish the manuscript. 


\section{AUTHORS' CONTRIBUTIONS}

HV, MvdL and TJ conceptualized this post-hoc study. PtK performed the statistical analysis. MGM drafted the first version of the manuscript. All authors participated in the interpretation of the results and revised it critically for important intellectual content. All authors read and approved the final version of the manuscript.

\section{ACKNOWLEDGEMENTS}

The authors thank all patients, rheumatology nurses and rheumatologists of the participating centers, the members of the Steering Committee consisting of Renée Allaart (University Medical Center Leiden), Annelies Boonen (Maastricht University Medical Center), Reinhard Bos (Medical Center Leeuwarden), Liesbeth Brouwer (University Medical Center Groningen), Alfons den Broeder (Sint Maartens Clinic), Danielle Gerlag (Amsterdam Medical Center), Mieke Hazes (Erasmus University Medical Center), Willem Lems (VU Medical Center), Dirkjan van Schaardenburg (Reade), Janneke Tekstra (University Medical Center Utrecht), and Harald Vonkeman (Arthritis Center Twente MST \& University ofTwente). The authors additionally thank Gerardine Willemsen (patients association), Huib Kooiman (Dutch Ministry of Health, Welfare \& Sports), and Benien Vingerhoeds (Netherlands Organisation for Health Research and Development). 


\section{REFERENCES}

1. Kvien TK. Epidemiology and burden of illness of rheumatoid arthritis. Pharmacoeconomics. 2004;22 Suppl 2:1-12.

2. Mclnnes IB, O'Dell JR. State-of-the-art: rheumatoid arthritis. Ann Rheum Dis. 2010;69:1898-906.

3. Chen Y-F, Jobanputra P, Barton P, Jowett S, Bryan S, Clark W, et al. A systematic review of the effectiveness of adalimumab, etanercept and infliximab for the treatment of rheumatoid arthritis in adults and an economic evaluation of their costeffectiveness. Health Technol Assess. 2006;10:1-229.

4. Wiens A, Venson R, Correr CJ, Otuki MF, Pontarolo R. Meta-analysis of the efficacy and safety of adalimumab, etanercept, and infliximab for the treatment of rheumatoid arthritis. Pharmacotherapy. 2010;30:33953.

5. Nam JL, Ramiro S, Gaujoux-Viala C, Takase K, Leon-Garcia M, Emery P, et al. Efficacy of biological disease-modifying antirheumatic drugs: a systematic literature review informing the 2013 update of the EULAR recommendations for the management of rheumatoid arthritis. Ann Rheum Dis. 2014;73:516-28.

6. Tanaka $Y$. Intensive treatment and treatment holiday of TNF-inhibitors in rheumatoid arthritis. Curr Opin Rheumatol. 2012;24:319-26.

7. Tanaka Y. Next stage of RA treatment: is TNF inhibitor-free remission a possible treatment goal? Ann Rheum Dis. 2013;72 Suppl 2:ii124-7.
8. Smolen JS, Landewé R, Breedveld FC, Buch M, Burmester G, Dougados M, et al. EULAR recommendations for the management of rheumatoid arthritis with synthetic and biological disease-modifying antirheumatic drugs: 2013 update. Ann Rheum Dis. 2014;73:492-509.

9. van Ingen ILA, Lamers-Karnebeek F, Jansen TL. Optimizing the expediency of TNFi in rheumatoid arthritis: offering a TNFi holiday in patients having reached lowdisease activity in the maintenance phase. Expert Opin Biol Ther. 2014;14:1761-7.

10. van den Broek M, Visser K, Allaart CF, Huizinga TWJ. Personalized medicine: predicting responses to therapy in patients with RA. Curr Opin Pharmacol. 2013;13:463-9.

11. Tweehuysen $\mathrm{L}$, van den Ende $\mathrm{CH}$, Beeren FMM, Been EMJ, van den Hoogen FHJ, den Broeder AA. Little Evidence for Usefulness of Biomarkers for Predicting Successful Dose Reduction or Discontinuation of a Biologic Agent in Rheumatoid Arthritis: A Systematic Review. Arthritis Rheumatol. 2017;69:301-8.

12. Saleem B, Keen H, Goeb V, Parmar R, Nizam S, Hensor EMA, et al. Patients with RA in remission on TNF blockers: when and in whom can TNF blocker therapy be stopped? Ann Rheum Dis. 2010;69:163642.

13. Tanaka Y, Takeuchi T, Mimori T, Saito K, Nawata M, Kameda H, et al. Discontinuation of infliximab after attaining low disease activity in patients with rheumatoid arthritis: 
RRR (remission induction by Remicade in RA) study. Ann Rheum Dis. 2010;69:128691.

14. van den Broek M, Klarenbeek NB, Dirven L, van Schaardenburg D, Hulsmans HMJ, Kerstens PJSM, et al. Discontinuation of infliximab and potential predictors of persistent low disease activity in patients with early rheumatoid arthritis and disease activity score-steered therapy: subanalysis of the BeSt study. Ann Rheum Dis. 2011;70:1389-94.

15. Kavanaugh A, Lee SJ, Curtis JR, Greenberg JD, Kremer JM, Soto L, et al. Discontinuation of tumour necrosis factor inhibitors in patients with rheumatoid arthritis in lowdisease activity: persistent benefits. Data from the Corrona registry. Ann Rheum Dis. 2014;0:1-6.

16. Haschka J, Englbrecht M, Hueber AJ, Manger B, Kleyer A, Reiser M, et al. Relapse rates in patients with rheumatoid arthritis in stable remission tapering or stopping antirheumatic therapy: interim results from the prospective randomised controlled RETRO study. Ann Rheum Dis. 2015.

17. Rech J, Hueber AJ, Finzel S, Englbrecht M, Haschka J, Manger B, et al. Prediction of disease relapses by multibiomarker disease activity and autoantibody status in patients with rheumatoid arthritis on tapering DMARD treatment. Ann Rheum Dis. 2015;75:1637-44.

18. Brocq O, Millasseau E, Albert C, Grisot C, Flory P, Roux C-H, et al. Effect of discontinuing TNFalpha antagonist therapy in patients with remission of rheumatoid arthritis. Joint Bone Spine. 2009;76:350-5.

19. Tracey D, Klareskog L, Sasso EH, Salfeld JG, Tak PP. Tumor necrosis factor antagonist mechanisms of action: a comprehensive review. Pharmacol Ther. 2008;117:244-79.

20. Arora T, Padaki R, Liu L, Hamburger AE, Ellison AR, Stevens SR, et al. Differences in binding and effector functions between classes of TNF antagonists. Cytokine. 2009;45:124-31.

21. Silva LCR, Ortigosa LCM, Benard G. Anti-TNF-a agents in the treatment of immune-mediated inflammatory diseases: mechanisms of action and pitfalls. Immunotherapy. 2010;2:817-33.

22. Marotte H, Cimaz R. Etanercept - TNF receptor and IgG1 Fc fusion protein: is it different from other TNF blockers? Expert Opin Biol Ther. 2014;14:569-72.

23. Kievit W, Adang EM, Fransen J, Kuper $H H$, van de Laar M a FJ, Jansen TL, et al. The effectiveness and medication costs of three anti-tumour necrosis factor alpha agents in the treatment of rheumatoid arthritis from prospective clinical practice data. Ann Rheum Dis. 2008;67:1229-34.

24. Singh JA, Christensen R, Wells GA, SuarezAlmazor ME, Buchbinder R, Lopez-Olivo MA, et al. Biologics for rheumatoid arthritis: an overview of Cochrane reviews. Cochrane database Syst Rev. 2009;:CD007848.

25. Ghiti Moghadam M, Vonkeman HE, Ten Klooster PM, Tekstra J, van Schaardenburg D, Starmans-Kool M, et al. Stopping Tumor Necrosis Factor Inhibitor Treatment in 
Patients With Established Rheumatoid Arthritis in Remission or With Stable Low Disease Activity: A Pragmatic Multicenter, Open-Label Randomized Controlled Trial. Arthritis Rheumatol (Hoboken, NJ). 2016;68:1810-7.

26. van der Maas A, Lie E, Christensen R, Choy E, de Man YA, van Riel P, et al. Construct and criterion validity of several proposed DAS28-based rheumatoid arthritis flare criteria: an OMERACT cohort validation study. Ann Rheum Dis. 2013;72:1800-5.

27. Centola M, Cavet G, Shen Y, Ramanujan S, Knowlton N, Swan KA, et al. Development of a multi-biomarker disease activity test for rheumatoid arthritis. PLoS One. 2013;8:e60635.

28. Ghiti Moghadam M, Lamers-Karnebeek FBG, Vonkeman HE, ten Klooster PM, Tekstra J, Schilder AM, et al. Multi-biomarker disease activity score as a predictor of disease relapse in patients with rheumatoid arthritis stopping TNF inhibitor treatment. PLoS One. 2018;13:e0192425. doi:10.1371/ journal.pone.0192425.

29. Prevoo ML, van 't Hof MA, Kuper $H H$, van Leeuwen MA, van de Putte LB, van Riel PL. Modified Disease Activity Scores that include twenty-eight-joint counts: development and validation in a prospective longitudinal study of patients with rheumatoid arthritis. Arthritis Rheum. 1995;38:44-8.

30. Curtis JR, van der Helm-van Mil AH, Knevel R, Huizinga TW, Haney DJ, Shen Y, et al. Validation of a novel multibiomarker test to assess rheumatoid arthritis disease activity. Arthritis Care Res (Hoboken). 2012;64:1794-803.

31. Tanaka Y, Hirata S, Kubo S, Fukuyo S, Hanami K, Sawamukai N, et al. Discontinuation of adalimumab after achieving remission in patients with established rheumatoid arthritis: 1-year outcome of the HONOR study. Ann Rheum Dis. 2015;74:389-95.

32. Tanaka Y, Smolen J, Jones H, Szumski A, Marshall L, Emery P. Baseline characteristics and treatment outcomes of patients who achieve deep remission in rheumatoid arthritis. Ann Rheum Dis. 2016;75 Suppl 2:188.2-189.

33. Rosenthal JA. Qualitative Descriptors of Strength of Association and Effect Size. J Soc Serv Res. 1996;21:37-59.

34. Hosmer DW, Lemeshow S. Applied Logistic Regression. 2nd edition. New York, NY: John Wiley \& Sons, Inc.; 2000.

35. Harigai M, Takeuchi T, Tanaka Y, Matsubara T, Yamanaka H, Miyasaka N. Discontinuation of adalimumab treatment in rheumatoid arthritis patients after achieving low disease activity. Mod Rheumatol. 2012;22:814-22.

36. van der Helm-van Mil AHM, Knevel R, Cavet G, Huizinga TWJ, Haney DJ. An evaluation of molecular and clinical remission in rheumatoid arthritis by assessing radiographic progression. Rheumatology (Oxford). 2013;52:839-46.

37. Hambardzumyan K, Bolce R, Saevarsdottir S, Cruickshank SE, Sasso EH, Chernoff D, et al. Pretreatment multi-biomarker disease activity score and radiographic progression 
PREDICTORS OF BIOLOGIC-FREE DISEASE CONTRL IN PATIENTS WITH RHEUMATOID ARTHRITIS AFTER STOPPING TUMOR NECROSIS FACTOR INHIBITOR TREATMENT

in early RA: results from the SWEFOT trial. Ann Rheum Dis. 2015;74:1102-9.

38. Li W, Sasso EH, van der Helm-van Mil AHM, Huizinga TWJ. Relationship of multibiomarker disease activity score and other risk factors with radiographic progression in an observational study of patients with rheumatoid arthritis. Rheumatology (Oxford). 2016;55:357-66. 



\section{Chapter}

\section{Multi-biomarker Disease Activity Score as a Predictor of Disease Relapse in Patients with Rheumatoid Arthritis Stopping TNF Inhibitor Treatment.}

Marjan Ghiti Moghadam,

Harlad E. Vonkeman,

Peter M. ten klooster,

Femke B.G. Lamers-Karnebeek,

Janneke Tekstra,

Annemarie M. Schilder,

Henk Visser,

Eric H. Sasso,

Willem F. Lems,

Dirkjan van Schaardenburg,
Robert Landew,

Hein J. Bernelot Moens,

Timothy R.D.J. Radstake,

Piet L.C.M. van Riel,

Mart A.F.J. van de Laar,

Tim L. Jansen,

on behalf of the Dutch National POET

Collaboration. 


\section{ABSTRACT}

\section{OBJECTIVE}

Successfully stopping or reducing treatment for patients with rheumatoid arthritis (RA) in low disease activity (LDA) may improve cost-effectiveness of care. We evaluated the multi-biomarker disease activity (MBDA) score as a predictor of disease relapse after discontinuation of TNF inhibitor (TNFi) treatment.

\section{METHODS}

439 RA patients who were randomized to stop TNFi treatment in the POET study were analyzed post-hoc. Three indicators of disease relapse were assessed over 12 months: 1) restarting TNFi treatment, 2) escalation of any DMARD therapy and 3) physicianreported flare. MBDA score was assessed at baseline. Associations between MBDA score and disease relapse were examined using univariate analysis and multivariate logistic regression.

\section{RESULTS}

At baseline, $50.1 \%, 35.3 \%$ and $14.6 \%$ of patients had low $(<30)$, moderate $(30-44)$ or high (>44) MBDA scores. Within 12 months, 49.9\% of patients had restarted TNFi medication, $59.0 \%$ had escalation of any DMARD and $57.2 \%$ had $\geq 1$ physician-reported flare. MBDA score was associated with each indicator of relapse. At least one indicator of relapse was observed in 59.5\%, $68.4 \%$ and $81.3 \%$ of patients with low, moderate or high MBDA scores, respectively $(P=0.004)$. Adjusted for baseline DAS28-ESR, disease duration, BMI and erosions, high MBDA scores were associated with increased risk for restarting TNFi treatment $(\mathrm{OR}=1.85)$, DMARD escalation $(\mathrm{OR}=1.99)$ and physician-reported flare $(\mathrm{OR}=2.00)$.

\section{CONCLUSION}

For RA patients with stable LDA who stopped TNFi, a high baseline MBDA score was independently predictive of disease relapse within 12 months. The MBDA score may be useful for identifying patients at risk of relapse after TNFi discontinuation.

\section{KEY WORDS}

Rheumatoid arthritis; biomarkers; multibiomarker; disease activity; prediction. 
Rheumatoid arthritis (RA) is a chronic inflammatory disease that can cause joint damage and physical disability (1). Early detection of RA and the availability of biologic agents have markedly improved outcomes in these patients ${ }^{(2)}$. Many studies have shown that the use of combinations of conventional synthetic DMARDs (csDMARDs) and biological DMARDs (bDMARDS) such as tumor necrosis factor inhibitors (TNFi) is effective for reaching and maintaining a state of low disease activity (LDA) or remission ${ }^{(3-5)}$. Once LDA or remission has been reached, patients often continue their combination therapy indefinitely. This practice may lead to overtreatment, as recent studies suggest that in some RA patients the more expensive TNFi can be tapered or stopped ${ }^{(6,7)}$. However, before implementing this therapeutic strategy in routine care, a validated predictor of disease relapse would be desirable (8).

Several studies have explored predictors of successful TNFi discontinuation. Results varied considerably, possibly due to differences in population, design and definitions of success, but most studies identified deep remission or lower disease activity at the time of discontinuation as a predictor ${ }^{(9-11)}$. Rheumatoid factor (RF) positivity, shorter disease duration, non-smoking and normal body mass index (BMI) may also be associated with better outcomes ${ }^{(10,12)}$. Although these studies all found that some patients could discontinue TNFi treatment without flaring, it remains a challenge to accurately predict which patients may successfully discontinue treatment and which are at higher risk of disease relapse ${ }^{(13)}$.

Studies of strategies for reducing DMARD treatment have mainly evaluated the predictive value of conventional clinical measures of disease activity and traditional biomarkers such as rheumatoid factor (RF) and anti-cyclic citrullinated peptide antibodies (ACPA) ${ }^{(10,12,14)}$. However, new biomarkers with interesting potential have become available. The multibiomarker disease activity (MBDA) blood test measures 12 serum proteins to produce a score that has been clinically validated as a measure of disease activity in patients with RA (15-17). MBDA scores have been shown to reflect current clinical disease activity and changes in disease activity over time, including treatment responses in RA patients treated with $\mathrm{TNFi}{ }^{(18)}$. The MBDA score assesses the activity of underlying biologic pathways rather than external signs and symptoms and may therefore provide information that is complementary to clinical assessment ${ }^{(16)}$. The MBDA score was a more accurate predictor of radiographic progression than the 28-joint Disease Activity Score with C-reactive protein (DAS28-CRP) or CRP, and it is often elevated in patients with low clinical disease activity or low CRP (18-22).

Recently, the MBDA score and ACPA were shown to be predictors of relapse for RA patients in stable remission when treatments with conventional DMARDs and/or biological DMARDs were tapered, and for some patients, subsequently stopped. Prediction was strongest 
when MBDA score and ACPA were combined ${ }^{(23)}$. This finding, in a study of drug tapering, suggests that the MBDA score may be capable of predicting the outcome of complete TNFi discontinuation. The aim of the present study was to examine the prognostic value of the MBDA score for disease relapse after discontinuation of TNFi in RA patients with stable LDA.

\section{METHODS}

\section{PATIENT COHORT}

Data used for these analyses were from the Dutch POET trial (the Netherlands Trial Register, number NTR3112) ${ }^{(24)}$. The study was approved by the Ethical Review Boards of all participating hospitals. Ethical approval for the study was granted by the Committee on Research involving Human Subjects, region Arnhem-Nijmegen (Commissie Mensgebonden Onderzoek regio Arnhem-Nijmegen) and local feasibility by all regional Ethical Committees. Patients were included from March 2012 to March 2014 and written informed consent was obtained from all patients. In this pragmatic, multicenter, open-label, randomized clinical trial, RA patients with stable LDA were randomized 2:1 to either stop or continue TNFi treatment. Patients were included from March 2012 to March 2014 and written informed consent was obtained from all patients. All participating patients were $\geq 18$ years old, had RA according to ACR 1987 criteria, and had received TNFi treatment for at least one year prior to inclusion. Patients had stable LDA for at least six months, defined as either two DAS28 with erythrocyte sedimentation rate (DAS28-ESR) measurements $<3.2$ or a rheumatologist clinical impression of remission or stable low disease activity and at least one CRP measurement $<10 \mathrm{mg} / \mathrm{L}$ in the six months prior to inclusion. Nearly all patients were receiving concomitant csDMARDs. In the 6 months prior to inclusion, no dosage changes were allowed for csDMARDs or corticosteroids.

In total, 531 patients were randomized to stop TNFi treatment in POET and followed for 12 months ${ }^{(24)}$. Concomitant treatment with csDMARDs was continued. If RA flared (DAS28ESR $\geq 3.2$ with a change in DAS28-ESR $>0.6)^{(25)}$, TNFi could be restarted at the discretion of the treating rheumatologist. Because the current study focused on the value of the MBDA score as a predictor of disease relapse after discontinuation of TNFi treatment, only data from patients randomized to the stop group were used. For the current analyses, baseline serum samples were available to measure MBDA scores for 439 of the 531 patients in the group that stopped TNFi treatment. 


\section{MEASUREMENTS}

Patients were evaluated by their treating rheumatologist and rheumatology nurse at baseline and at least once every 3 months thereafter, for a period of one year. Baseline measures included: age, sex, weight, height, disease duration, medication use, rheumatoid factor (RF) and ACPA status, concomitant use of CSDMARDs and, for this post hoc analysis, the MBDA score. Clinical measurements were performed at every scheduled or unscheduled visit and included the ESR (mm/h), CRP (mg/l), 28-joint tender joint count (TJC28), 28-joint swollen joint count (SJC28), and a patient-reported assessment of general health on a $100 \mathrm{~mm}$ visual analog scale (VAS-GH). These component measures were combined to calculate DAS28-ESR ${ }^{(26)}$. Physician-reported flares and all changes in medication were recorded throughout the study.

\section{SERUM BIOMARKER MEASUREMENT AND MBDA SCORE CALCULATION}

Serum samples were stored at $-40^{\circ} \mathrm{C}$ from time of preparation until transfer to Crescendo Bioscience (South San Francisco, CA, USA), where they were stored at $-70^{\circ} \mathrm{C}$ or lower until biomarker concentration testing was performed in the Crescendo clinical laboratory, which is certified under the CMS Clinical Laboratory Improvement Amendments and accredited by the College of American Pathologists for determination of Vectra ${ }^{\circledR}$ DA scores. Biomarker concentrations were measured by electrochemiluminescence-based multiplexed immunoassays (Meso Scale Discovery, Rockville, MD, USA). The MBDA algorithm combines the concentrations of 12 biomarkers (vascular cell adhesion molecule-1 [VCAM-1], epidermal growth factor [EGF], vascular endothelial growth factor-A [VEGF-A], interleukin [IL]-6, tumor necrosis factor-receptor type 1 [TNF-RI], matrix metalloproteinase [MMP]-1, MMP-3, cartilage glycoprotein 39 [YKL-40], leptin, resistin, serum amyloid A [SAA], and CRP)(15) to generate the MBDA score on a scale of 1 to 100 , with previously validated categories for low (<30), moderate (30 to 44 ) and high (>44) disease activity ${ }^{(16)}$.

\section{STATISTICAL ANALYSIS}

Baseline demographic and disease-related characteristics were compared between the 439 patients with a baseline MBDA assessment and the 92 patients without an MBDA assessment using independent samples t-tests and Mann-Whitney $U$ tests for normally and non-normally distributed continuous variables, and Pearson $x^{2}$ tests for categorical variables. Disease relapse was defined three ways, using the criteria of: 1) restarting TNFi treatment, 2) any DMARD medication escalation and 3) physician-reported flare. DMARD medication escalation was defined as restarting TNFi treatment or starting or increasing the dosing of any bDMARD or csDMARD (including corticosteroids) ${ }^{(24)}$. Baseline characteristics of patients who did and did not meet the different criteria for relapse within the 12-month follow-up period were first compared using univariate logistic regression analyses. Patients 
who dropped out before 12 months of follow-up without meeting a criterion for relapse were counted in this analysis with those who continued to have a response. For each criterion for relapse, the proportions of patients in the low (<30), moderate (30-44) and high (>44) MBDA score groups who relapsed were compared by univariate Pearson $x^{2}$ tests with Bonferroni adjustment for the number of comparisons $(P<(0.05 / 3=) 0.017)$. Additional sensitivity analyses were performed limited to those patients that were included based on two available DAS28 scores <3.2in the six months prior to inclusion, those who met the inclusion criteria for stable LDA but had moderate DAS28-ESR at baseline, and those who were in remission at baseline (DAS28 scores <2.6). One-year relapse-free survival was examined for the low, moderate, and high MBDA score groups using Kaplan-Meier survival curves; patients who dropped out early without disease relapse were censored at the time of withdrawal. Between-group differences in survival were tested by pairwise log-rank tests, again with Bonferroni adjustment for the number of comparisons $(P<0.017)$. Based on the results of the univariate $x^{2}$ tests and Kaplan-Meier survival analyses, baseline MBDA scores were dichotomized as high (>44) vs. moderate-to-low ( $\leq 44)$. Cox proportional hazard regressions were used to estimate the hazard ratio (HR), which may be interpreted as a relative risk, of high vs. moderate-to-low MBDA score for the time to relapse. Next, univariate and multivariable logistic regression analyses were performed to evaluate the association between disease relapse within 12 months and high baseline MBDA score in terms of unadjusted odds ratios (ORs), ORs adjusted for baseline DAS28-ESR score, and ORs further adjusted for all other variables that were significantly $(P<0.05)$ associated witha relapse criterion in the univariate logistic regression analyses. Final sensitivity analyses were performed in which all patients with a missing visit (missing DAS28 score at 3, 6, 9, or 12 months) were counted as a flare on all flare criteria. All analyses were performed using SPSS version 22. 


\section{RESULTS}

\section{DEMOGRAPHIC AND CLINICAL DATA AT BASELINE}

From the 531 patients who were randomized to stop TNFi treatment in POET, baseline serum samples were available for MBDA testing for 439 patients. Among these patients, 356 (81.1\%) were included based on at least two available DAS28 scores <3.2, and 83 (18.9\%) were included based on the rheumatologist clinical impression of remission or stable low disease activity in combination with at least one available CRP value $<10 \mathrm{mg} / \mathrm{L}$. Baseline demographic and clinical data were similar between patients with or without a baseline MBDA sample (Table 1). Patients were typically older Dutch Caucasian females, with longstanding RF-positive, erosive RA. Most patients were receiving their first TNFi, with $51.3 \%$ of 439 patients receiving adalimumab, $40.1 \%$ receiving etanercept and $8.6 \%$ receiving infliximab, certolizumab or golimumab. Clinical disease activity was generally low, in accordance with study inclusion criteria, and 349 (79.5\%) patients were in remission (DAS28-ESR <2.6) at baseline. Seventeen (3.9\%) patients dropped out during the first 12 months of follow-up because of their own decision to drop out $(n=13)$ or presence of a comorbidity $(n=4)$.

Table 1. Baseline characteristics of POET patients grouped according to sample availability for MBDA testing

\begin{tabular}{lccc}
\hline & $\begin{array}{c}\text { MBDA } \\
\text { sample } \\
\text { Characteristic (N=531) }\end{array}$ & $\begin{array}{c}\text { No MBDA sample } \\
\text { (n=92) }\end{array}$ & P \\
\hline Female, n (\%) & $296(67.4 \%)$ & $66(71.7 \%)$ & 0.419 \\
Age (yrs.), mean (SD) & $59.8(10.8)$ & $61.7(10.6)$ & 0.137 \\
Disease duration (yrs.), median (IQR) & $10(6-17)$ & $9(6-16)$ & 0.535 \\
BMI, mean (SD) & $25.9(4.3)$ & $25.9(4.0)$ & 0.854 \\
RF positive, n (\%) & $270(67.3 \%)$ & $58(68.2 \%)$ & 0.872 \\
ACPA positive, n (\%) & $277(69.1 \%)$ & $55(64.7 \%)$ & 0.431 \\
Erosive disease, n (\%) & $53(62.4 \%)$ & 0.932 \\
ESR, median (IQR) & $252(62.8 \%)$ & $9.5(5-18)$ & 0.638 \\
CRP, median (IQR) & $9.0(5-17)$ & $3(1-5.8)$ & 0.388 \\
TJC28, median (IQR) & $2(1-5)$ & $0(0-0)$ & 0.043 \\
SJC28, median (IQR) & $0(0-1)$ & $0(0-1)$ & 0.328 \\
PGA, median (IQR) & $0(0-0)$ & $20.4(5.0-23.4)$ & 0.455 \\
DAS28-ESR & $20.7(9.0-28.1)$ & $1.9(0.7)$ & 0.549 \\
MBDA score, mean (SD) & $2.0(0.8)$ & - & - \\
\hline
\end{tabular}


Table 1 CONTINUED. Baseline characteristics of POET patients grouped according to sample availability for MBDA testing

\begin{tabular}{|c|c|c|c|}
\hline Characteristic $(\mathrm{N}=531)$ & $\begin{array}{c}\text { MBDA } \\
\text { sample } \\
(n=439)\end{array}$ & $\begin{array}{l}\text { No MBDA sample } \\
\qquad(n=92)\end{array}$ & $\mathbf{P}$ \\
\hline Number of TNFi, n (\%) & & & 0.819 \\
\hline $1^{\text {st }}$ & 379 (86.5\%) & 80 (87.0\%) & \\
\hline $2^{\text {nd }}$ & 50 (11.4\%) & $11(12.0 \%)$ & \\
\hline $3^{\text {rd }}$ & $9(2.1 \%)$ & $1(1.1 \%)$ & \\
\hline \multicolumn{4}{|l|}{ csDMARD, n (\%) } \\
\hline Methotrexate & 382 (87.0\%) & $77(83.7)$ & 0.581 \\
\hline Other csDMARD & 35 (8.0\%) & 8 (8.7\%) & \\
\hline No DMARD & 22 (5.0\%) & 7 (7.6\%) & \\
\hline
\end{tabular}

$\mathrm{TNFi}=$ tumor necrosis factor-alpha inhibitors; DAS28 = disease activity score in 28 joints; BMI = body mass index; RF = rheumatoid factor; $\mathrm{ACPA}=$ anti-cyclic citrullinated peptide antibodies; $\mathrm{ESR}$ = erythrocyte sedimentation rate; $\mathrm{CRP}$ = C-reactive protein; TJC28 = 28-joint tender joint count; SJC28 = 28-joint swollen joint count; PGA = patient global assessment; MBDA = multi-biomarker disease activity; csDMARD = conventional synthetic disease modifying antirheumatic drug; SD (standard deviation); IQR (interquartile range).

\section{ASSOCIATION BETWEEN BASELINE MBDA SCORE AND DISEASE RELAPSE}

Baseline MBDA scores were low (<30) in 220 (50.1\%) patients, moderate (30-44) in 155 (35.3\%) patients, and high (>44) in 64 (14.6\%). Clinical disease activity at baseline, as measured with the DAS28-ESR, was low in 413 (94.1\%) patients and moderate in 26 (5.9\%) patients. Within 12 months, 219 patients (49.9\%) had restarted TNFi treatment, 259 (59.0\%) patients had escalated any DMARD, and 251 (57.2\%) had experienced at least one physician-reported flare. There was no significant difference in the proportion of patients experiencing a relapse between those who were included based on available DAS28 scores and those who were included based on the rheumatologist clinical impression and the CRP value (TNFi restart: 51.4\% vs. 43.4\%, P = 0.188; medication escalation: $60.1 \%$ vs. 54.2\%, $P=0.325$; physician-reported flare: $57.3 \%$ vs. 56.6\%, $P=0.911)$.

There was considerable overlap of the different relapse groups (Figure 1). For example, among the 289 patients who met at least one criterion for relapse, only 12 (4.2\%) restarted TNFi treatment without also having a physician-reported flare, while 44 (15.2\%) patients had a physician-reported flare but did not restart TNFi treatment; 207 out of 289 (71.6\%) of the patients who relapsed met all three criteria. One hundred fifty (34.2\%) patients in the overall cohort $(\mathrm{N}=439)$ completed one year without meeting any of the three criteria for relapse. 


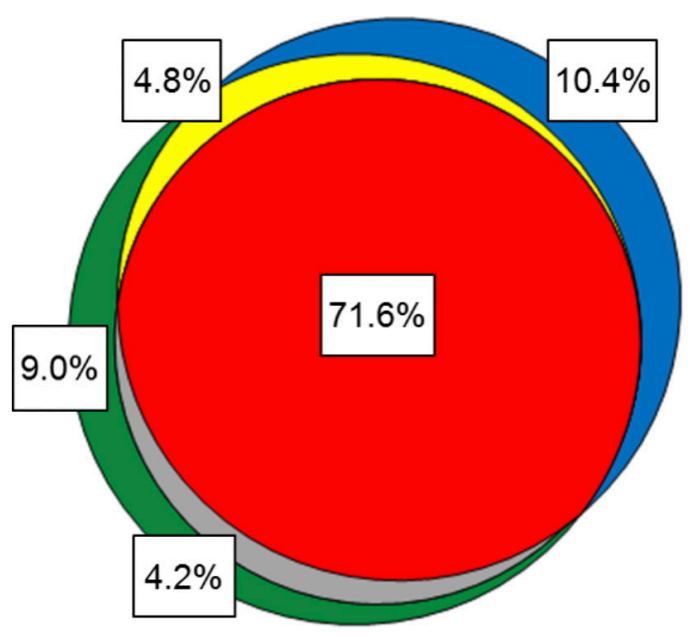

Figure 1. Venn diagram of patients meeting criteria for disease relapse.

Red $=\mathrm{TNFi}$ restart; green $=$ medication escalation; blue $=$ physician-reported flare; yellow $=$ overlap medication escalation / physician-reported flare; grey = overlap medication escalation / TNFi restart. Percentages are for the 289 patients who met at least one of the three criteria of disease relapse.

High MBDA scores (>44) at baseline were univariately associated with significantly (Bonferroni adjusted $\mathrm{P}<0.017$ ) greater proportions of patients meeting the criteria for disease relapse (Table 2). At least one criterion of relapse was met within 12 months of TNFi discontinuation by $59.5 \%, 68.4 \%$ and $81.3 \%$ of patients with low, moderate, or high baseline MBDA scores, respectively $(P=0.004)$. Differences in the cumulative 12 -month proportions of patients with relapse and the times to event were relatively small between patients with low or moderate MBDA scores, but patients with high MBDA scores were clearly at increased risk.

Table 2. Disease relapse by three criteria at $\mathbf{1 2}$ months for patients classified by baseline MBDA score

\begin{tabular}{lccccc}
\hline Criterion for relapse & Total & $\begin{array}{c}\text { Low (<30) } \\
\mathbf{n = 2 2 0}\end{array}$ & $\begin{array}{c}\text { Moderate (30-44) } \\
\mathbf{n = 1 5 5}\end{array}$ & $\begin{array}{c}\text { High (>44) } \\
\mathbf{n = 6 4}\end{array}$ & P \\
\hline TNFi restart & 219 & $102(46.4 \%)$ & $74(47.7 \%)$ & $43(67.2 \%)$ & 0.011 \\
Medication escalation & 259 & $117(53.2 \%)$ & $92(59.4 \%)$ & $50(78.1 \%)$ & 0.002 \\
Physician-reported flare & 251 & $116(52.7 \%)$ & $87(56.1 \%)$ & $48(75.0 \%)$ & 0.006 \\
Any criterion & 289 & $131(59.5 \%)$ & $106(68.4 \%)$ & $52(81.3 \%)$ & 0.004 \\
\hline
\end{tabular}

Any criterion = TNFi re-initation, medication escalation, or physician-reported flare. P-value by Pearson $x^{2}$ test. Total $\mathrm{N}=439$ 
Differences in disease relapse were very similar when limited to those patients that were included based on two available DAS28 scores <3.2in the six months prior to inclusion (Supplementary Table 1), although no longer significant for TNFi restart after Bonferroni adjustment. Similar results were also obtained in a sensitivity analysis that excluded the 26 patients who met the inclusion criteria for stable LDA but had moderate DAS28-ESR at baseline (Supplementary Table 2). Among patients in remission at baseline (DAS28ESR <2.6), differences between MBDA categories were slightly less pronounced and not significant after Bonferroni correction (Supplementary Table 3).

Univariate Kaplan-Meier survival analyses confirmed the significantly decreased oneyear relapse-free survival in patients with high baseline MBDA scores (Figure 2). Pairwisedifferences in one-year relapse-free survival were not significant between patients with low or moderate MBDA scores, but patients with high MBDA scores had significantly (Bonferroni adjusted $\mathrm{P}<0.017$ ) worse relapse-free survival than patients with either moderate or Iow MBDA scores for all three criteria of relapse. The HRs of high vs. moderate-to-low baseline MBDA scores were 1.61 (95\% Cl 1.15-2.25, P = 0.005) for TNFi restart, $1.66(95 \% \mathrm{Cl} 1.22-2.26, \mathrm{P}=0.001)$ for medication escalation, and $1.64(95 \% \mathrm{Cl}$ $1.20-2.25, P=0.002$ ) for physician-reported flare. 

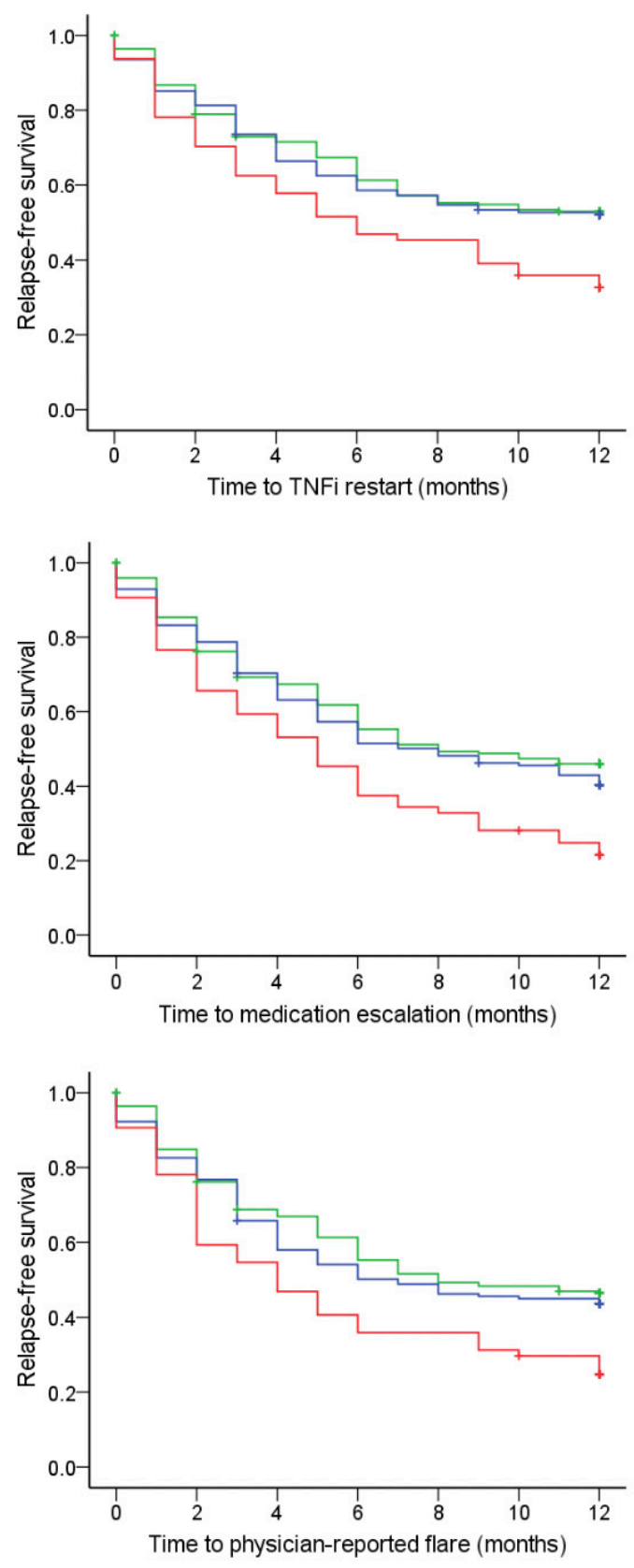

Figure 2. Kaplan-Meier survival curves.

Low MBDA scores ( $<30$, green; $n=220$ ), moderate MBDA scores (30-44, blue; $n=155$ ), high MBDA scores ( $>40$, red; $n=64$ ) for three definitions of disease relapse. TNFi restart (top), medication escalation (middle) and physician-reported flare (bottom) 


\section{UNIVARIATE AND MULTIVARIATE ANALYSIS OF RISK FACTORS FOR DISEASE RELAPSE AT 12 MONTHS}

In univariate logistic regression analyses, greater disease duration was significantly associated with all three criteria for disease relapse at 12 months (ORs 1.04-1.05, 95\% Cl 1.01-1.08) (Supplementary Table 4). Higher BMI was associated with increased odds of restarting TNFi (OR 1.05, 95\% Cl 1.01-1.10), medication escalation (OR 1.07, 95\% Cl 1.02-1.13) and any criterion (OR 1.06, 95\% Cl 1.01-1.12). Erosive disease was significantly associated with restarting TNFi (OR 1.62, 95\% Cl 1.08-2.44), but not with the other two criteria. Baseline DAS28-ESR was significantly associated with medication escalation (OR 1.57, 95\% Cl 1.212.04), physician-reported flare (OR 1.36, 95\% Cl 1.06-1.75) and any criterion (OR 1.65, 95\% $\mathrm{Cl} 1.26-2.17)$, but not with restarting TNFi treatment (OR 1.25, 95\% Cl 0.98-1.60).

In multivariate analysis with adjustment for baseline DAS28-ESR, disease relapse after stopping TNFi treatment was more than twice as likely to occur in patients with a high baseline MBDA score, regardless of the relapse criterion used (Table 3). After adjusting for baseline DAS28-ESR, disease duration, BMI and erosions, high baseline MBDA score remained a significant independent predictor of disease relapse by all three criteria (Table 3). However, it did not remain significantly associated with the composite definition of relapse in the fully adjusted model. In a final sensitivity analysis in which all patients with a missing visit were counted as a flare, high MBDA score remained a significant predictor of clinical flare and a marginally significant predictor of medication escalation and TNFirestart (Supplementary Table 5). 
Table 3. Univariate and multivariate analyses of high (>44) versus moderate or low baseline MBDA score as a predictor of disease relapse at 12 months

\begin{tabular}{|c|c|c|c|c|c|c|}
\hline \multirow[b]{2}{*}{ Criterion for relapse } & \multicolumn{2}{|c|}{ Unadjusted } & \multicolumn{2}{|l|}{ Adjusted } & \multicolumn{2}{|c|}{ Fully adjusted } \\
\hline & OR (95\% Cl) & $\mathbf{P}$ & OR (95\% Cl) & $\mathbf{P}$ & OR (95\% Cl) & $\mathbf{P}$ \\
\hline \multicolumn{7}{|l|}{ TNFi restart } \\
\hline MBDA $>44$ & $2.32(1.32-4.05)$ & 0.003 & $2.17(1.23-3.83)$ & 0.008 & $1.85(1.00-3.40)$ & 0.049 \\
\hline DAS28-ESR & & & $1.17(0.91-1.51)$ & 0.219 & $1.10(0.84-1.45)$ & 0.474 \\
\hline Disease duration & & & & & 1.05 (1.02-1.08) & $<0.001$ \\
\hline BMI & & & & & $1.06(1.01-1.11)$ & 0.031 \\
\hline Erosive & & & & & $1.30(1.00-3.40)$ & 0.248 \\
\hline \multicolumn{7}{|l|}{ Medication escalation } \\
\hline MBDA $>44$ & $2.84(1.52-5.31)$ & 0.001 & $2.44(1.29-4.62)$ & 0.006 & 1.99 (1.01-3.94) & 0.047 \\
\hline DAS28-ESR & & & $1.47(1.13-1.92)$ & 0.004 & $1.48(1.11-1.97)$ & 0.008 \\
\hline Disease duration & & & & & $1.04(1.01-1.06)$ & 0.011 \\
\hline BMI & & & & & $1.07(1.01-1.13)$ & 0.014 \\
\hline Erosive & & & & & 1.24 (0.79-1.97) & 0.353 \\
\hline \multicolumn{7}{|l|}{ Physician-reported flare } \\
\hline MBDA $>44$ & $2.54(1.39-4.64)$ & 0.002 & $2.31(1.25-4.25)$ & 0.007 & $2.00(1.06-3.77)$ & 0.033 \\
\hline DAS28-ESR & & & 1.27 (0.98-1.65) & 0.069 & $1.20(0.92-1.58)$ & 0.184 \\
\hline Disease duration & & & & & 1.04 (1.01-1.06) & 0.007 \\
\hline $\mathrm{BMI}$ & & & & & 1.03 (0.98-1.08) & 0.228 \\
\hline Erosive & & & & & $1.08(0.69-1.68)$ & 0.784 \\
\hline \multicolumn{7}{|l|}{ Any criterion } \\
\hline MBDA $>44$ & $2.52(1.30-4.89)$ & 0.006 & $2.12(1.08-4.16)$ & 0.029 & $1.68(0.83-3.40)$ & 0.147 \\
\hline DAS28-ESR & & & $1.56(1.18-2.07)$ & 0.002 & $1.54(1.14-2.07)$ & 0.005 \\
\hline Disease duration & & & & & $1.04(1.01-1.07)$ & 0.010 \\
\hline BMI & & & & & 1.06 (1.00-1.12) & 0.037 \\
\hline Erosive & & & & & $1.25(0.78-3.40)$ & 0.347 \\
\hline
\end{tabular}

DAS28-ESR, disease duration and BMI were analyzed as continuous variables; MBDA score (>44) and erosive (yes/ no) were analyzed as categorical variables. Adjusted = adjusted for DAS28. Fully adjusted = adjusted for DAS28, disease duration, BMI and erosions. Any criterion includes patients with TNFi re-initation, medication escalation or physician-reported flare. Total $\mathrm{N}=439$. See Table 2 for $\mathrm{n}$-values by relapse criterion. 


\section{DISCUSSION}

Our analyses of the POET study show that, for RA patients in remission or stable LDA, a high MBDA score at the time of TNFi discontinuation was significantly associated with disease relapse during the next 12 months. Over $80 \%$ of patients with a high baseline MBDA score relapsed according to at least one of the three criteria we used. This rate of relapse was substantially higher for patients with low or moderate MBDA scores, suggesting that patients with low clinical disease activity and high MBDA scores may have inflammation that is partly or entirely subclinical. Several other studies have found the MBDA score to be frequently elevated when conventional clinical measures indicate remission or LDA $(19,20,22,23)$. Moreover, such patients were at increased risk for progressive joint damage ${ }^{(19,20,22)}$. Consequently, discontinuation of TNFi treatment in POET may have allowed a resurgence of residual inflammation and subsequent clinical relapse ${ }^{(23)}$. Our finding that the MBDA score was a predictor of relapse independently of DAS28-ESR suggests that it may be a clinically useful tool for identifying patients who are at increased risk of unsuccessful TNFi discontinuation.

Although high MBDA was an independent predictor of three predefined criteria for relapse (TNFi restart, medication escalation, and physician-reported flare), it should be noted that it did not remain significantly associated with meeting any criterion of flare when adjusting for all other potential predictors, including DAS28 score. Previous studies have explored predictors of disease relapse after TNFi discontinuation and, although results varied considerably, greater clinical disease activity at the time of discontinuation has been identified as a predictor ${ }^{(9-11)}$. Likewise, our study found that higher baseline DAS28-ESR scores were associated with two criteria for disease relapse, but not with the criterion of restarting TNFi treatment.

In other studies, RF positivity, shorter disease duration, non-smoking and normal body mass index (BMI) have been associated with successful TNFi discontinuation ${ }^{(10,12)}$. In the current study, higher BMI scores were univariately associated with increased odds of meeting two criteria of disease relapse but not physician-reported flare, and longer disease duration was a strong predictor for all three definitions of disease relapse. Erosive disease was univariately associated with TNFi restart. Neither positivity for RF nor ACPA was associated with disease relapse.

We previously demonstrated that RA patients in remission or stable low disease activity, as defined by the DAS28-ESR, had a relapse risk of approximately 50\% within 12 months of discontinuing TNFi treatment in the POET study ${ }^{(24)}$. With such a high likelihood of relapsing, 
it may be helpful to have an effective tool to predict the outcome of treatment withdrawal. The MBDA score has been shown to correlate significantly with the DAS28-ESR, DAS28CRP, simplified disease activity index (SDAl) and clinical disease activity index (CDAI), both overall and in seronegative and seropositive RA patients ${ }^{(16,27,28)}$. The MBDA score was an independent predictor of disease relapse in a study of RA patients in clinical remission who tapered, and in one arm of the study stopped, all RA therapy, including cSDMARDs and bDMARDs ${ }^{(18)}$. The present study is the first to demonstrate the utility of the MBDA score as a predictor for risk of disease relapse in RA patients who discontinued TNFi treatment at baseline.

Patients with high MBDA had an odds ratio of approximately 2 for experiencing a relapse as defined by the three criteria. However, the proportion of patients with low or moderate MBDA scores who still relapsed within 12 months was also quite high, which may limit the utility of the MBDA to guide TNFi discontinuation in clinical practice. Additionally, since odds ratios tend to overestimate the probability of frequent events (the overall prevalence of relapse ranged from approximately $50 \%-60 \%$ on the different criteria), the hazard ratios or relative risks of around 1.6 found in the one-year survival analyses may be a more appropriate and precise estimate of risk for relapse associated with high MBDA.

Our study has several strengths. The data we analyzed were collected in POET, the largest randomized controlled trial on stopping TNFi in RA patients in remission or stable Iow disease activity to date. The MBDA score was measured in an unbiased selection of 439 patients in the stop group, comprising 83\% of those who stopped TNFi treatment in POET. Most patients in our study had long disease duration (i.e., established RA) and an average age of 60 years, which is representative of the TNFi-using RA population in The Netherlands. Our finding that MBDA score was a predictor of relapse risk is strengthened by our having used 3 different definitions of disease relapse: 1) restarting TNFi treatment, 2) escalation of any DMARD therapy and 3) physician-reported flare, which identified more relapses than with any one criterion alone. MBDA scores at baseline were predictive of each definition of disease relapse.

A limitation of the study is that MBDA score was measured only at baseline. Longitudinal measurements may have provided insight into the effect of TNFi discontinuation on MBDA scores over time, and the potential for early change in MBDA score to predict relapse. Although only the MBDA score has been validated for measuring disease activity, it remains to be explored if any of the 12 biomarkers in the MBDA score might individually have ability to predict disease relapse. 
In conclusion, for RA patients in remission or stable LDA, a high baseline MBDA score was frequently observed and was found to be an independent predictor of disease relapse within 12 months of TNFi discontinuation. These results suggest that the MBDA score may be a clinically useful tool for identifying subgroups of patients who have an increased risk of relapse when stopping TNFi treatment. These data should be confirmed in other studies.

\section{ACKNOWLEDGEMENTS}

The authors thank all patients, rheumatology nurses and rheumatologists of the participating centers, the members of the Steering Committee consisting of Renée Allaart (University Medical Center Leiden), Annelies Boonen (Maastricht University Medical Center), Reinhard Bos (Medical Center Leeuwarden), Liesbeth Brouwer (University Medical Center Groningen), Alfons den Broeder (Sint Maartens clinic), Danielle Gerlag (Amsterdam Medical Center), Mieke Hazes (Erasmus University Medical Center), Willem Lems (VU Medical Center), Dirkjan van Schaardenburg (Reade), Janneke Tekstra (University Medical Center Utrecht), and Harald Vonkeman (Arthritis Center Twente MST \& University of Twente), and Gerardine Willemsen (patients association), Huib Kooiman (Dutch Ministry of Health, Welfare \& Sports (VWS)), and Benien Vingerhoeds (Netherlands Organisation for Health Research and Development (ZonMW)). 


\section{REFERENCES}

1. Kvien TK. Epidemiology and burden of illness of rheumatoid arthritis. Pharmacoeconomics. 2004;22: 1-12.

2. Mclnnes IB, O'Dell JR. State-of-theart: rheumatoid arthritis. Ann Rheum Dis. 2010;69: 1898-906. doi:10.1136/ ard. 2010.134684

3. Chen Y-F, Jobanputra P, Barton P, Jowett S, Bryan S, Clark W, et al. A systematic review of the effectiveness of adalimumab, etanercept and infliximab for the treatment of rheumatoid arthritis in adults and an economic evaluation of their costeffectiveness. Health Technol Assess. 2006;10: iii-iv, xi-xiii, 1-229.

4. Wiens A, Venson R, Correr CJ, Otuki MF, Pontarolo R. Meta-analysis of the efficacy and safety of adalimumab, etanercept, and infliximab for the treatment of rheumatoid arthritis. Pharmacotherapy. 2010;30: 33953. doi:10.1592/phco.30.4.339

5. Nam JL, Ramiro S, Gaujoux-Viala C, Takase K, Leon-Garcia M, Emery P, et al. Efficacy of biological disease-modifying antirheumatic drugs: a systematic literature review informing the 2013 update of the EULAR recommendations for the management of rheumatoid arthritis. Ann Rheum Dis. 2014;73: 516-28. doi:10.1136/ annrheumdis-2013-204577

6. Tanaka Y. Intensive treatment and treatment holiday of TNF-inhibitors in rheumatoid arthritis. Curr Opin Rheumatol. 2012;24: 319-26. doi:10.1097/ BOR.0b013e3283524e4c

7. Tanaka Y. Next stage of RA treatment: is
TNF inhibitor-free remission a possible treatment goal? Ann Rheum Dis. 2013;72 Suppl 2: ii124-7. doi:10.1136/ annrheumdis-2012-202350

8. van Ingen ILA, Lamers-Karnebeek $F$, Jansen TL. Optimizing the expediency of TNFi in rheumatoid arthritis: offering a TNFi holiday in patients having reached lowdisease activity in the maintenance phase. Expert Opin Biol Ther. 2014;14: 1761-7. doi:1 $0.1517 / 14712598.2014 .955009$

9. Saleem B, Keen H, Goeb V, Parmar R, Nizam S, Hensor EMA, et al. Patients with RA in remission on TNF blockers: when and in whom can TNF blocker therapy be stopped? Ann Rheum Dis. 2010;69: 163642. doi:10.1136/ard.2009.117341

10. Tanaka Y, Takeuchi T, Mimori T, Saito K, Nawata M, Kameda H, et al. Discontinuation of infliximab after attaining low disease activity in patients with rheumatoid arthritis: RRR (remission induction by Remicade in RA) study. Ann Rheum Dis. 2010;69: 128691. doi:10.1136/ard.2009.121491

11. Harigai M, Takeuchi T, Tanaka Y, Matsubara T, Yamanaka H, Miyasaka N. Discontinuation of adalimumab treatment in rheumatoid arthritis patients after achieving low disease activity. Mod Rheumatol. 2012;22: 814-22. doi:10.1007/s10165-011-0586-5

12. Kavanaugh A, Lee SJ, Curtis JR, Greenberg JD, Kremer JM, Soto L, et al. Discontinuation of tumour necrosis factor inhibitors in patients with rheumatoid arthritis in low-disease activity: persistent benefits. Data from the Corrona registry. 
Ann Rheum Dis. 2014;0: 1-6. doi:10.1136/ annrheumdis-2014-206435

13. van den Broek M, Visser K, Allaart CF, Huizinga TWJ. Personalized medicine: predicting responses to therapy in patients with RA. Curr Opin Pharmacol. 2013;13: 463-9. doi:10.1016/j.coph.2013.03.006

14. van den Broek M, Klarenbeek NB, Dirven L, van Schaardenburg D, Hulsmans HMJ, Kerstens PJSM, et al. Discontinuation of infliximab and potential predictors of persistent low disease activity in patients with early rheumatoid arthritis and disease activity score-steered therapy: subanalysis of the BeSt study. Ann Rheum Dis. 2011;70: 1389-94. doi:10.1136/ard.2010.147751

15. Centola M, Cavet G, Shen Y, Ramanujan S, Knowlton N, Swan KA, et al. Development of a multi-biomarker disease activity test for rheumatoid arthritis. PLoS One. 2013;8: e60635. doi:10.1371/journal.pone.0060635

16. Curtis JR, van der Helm-van Mil AH, Knevel R, Huizinga TW, Haney DJ, Shen $Y$, et al. Validation of a novel multibiomarker test to assess rheumatoid arthritis disease activity. Arthritis Care Res (Hoboken). 2012;64: 1794-803. doi:10.1002/acr.21767

17. Eastman PS, Manning WC, Qureshi F, Haney D, Cavet G, Alexander C, et al. Characterization of a multiplex, 12-biomarker test for rheumatoid arthritis. J Pharm Biomed Anal. 2012;70: 415-24. doi:10.1016/j.jpba.2012.06.003

18. Hirata S, Li W, Defranoux N, Cavet G, Bolce $\mathrm{R}$, Yamaoka $\mathrm{K}$, et al. A multi-biomarker disease activity score tracks clinical response consistently in patients with rheumatoid arthritis treated with different anti-tumor necrosis factor therapies: A retrospective observational study. Mod Rheumatol. 2015;25: 344-9. doi:10.3109/14 397595.2014 .958893

19. van der Helm-van Mil AHM, Knevel R, Cavet G, Huizinga TWJ, Haney DJ. An evaluation of molecular and clinical remission in rheumatoid arthritis by assessing radiographic progression. Rheumatology (Oxford). 2013;52: 839-46. doi:10.1093/ rheumatology/kes378

20. Hambardzumyan K, Bolce R, Saevarsdottir S, Cruickshank SE, Sasso EH, Chernoff D, et al. Pretreatment multi-biomarker disease activity score and radiographic progression in early RA: results from the SWEFOT trial. Ann Rheum Dis. 2015;74: 1102-1109. doi:10.1136/annrheumdis-2013-204986

21. Lee $Y C$, Hackett J, Frits M, lannaccone CK, Shadick NA, Weinblatt ME, et al. Multibiomarker disease activity score and C-reactive protein in a cross-sectional observational study of patients with rheumatoid arthritis with and without concomitant fibromyalgia. Rheumatology (Oxford). 2016;55: 640-8. doi:10.1093/ rheumatology/kev388

22. Li W, Sasso EH, van der Helm-van Mil AHM, Huizinga TWJ. Relationship of multibiomarker disease activity score and other risk factors with radiographic progression in an observational study of patients with rheumatoid arthritis. Rheumatology (Oxford). 2016;55: 357-66. doi:10.1093/ 
rheumatology/kev341

23. Rech J, Hueber AJ, Finzel S, Englbrecht M, Haschka J, Manger B, et al. Prediction of disease relapses by multibiomarker disease activity and autoantibody status in patients with rheumatoid arthritis on tapering DMARD treatment. Ann Rheum Dis. 2015;75: 1637-44. doi:10.1136/ annrheumdis-2015-207900

24. Ghiti Moghadam M, Vonkeman HE, Ten Klooster PM, Tekstra J, van Schaardenburg D, Starmans-Kool M, et al. Stopping Tumor Necrosis Factor Inhibitor Treatment in Patients With Established Rheumatoid Arthritis in Remission or With Stable Low Disease Activity: A Pragmatic Multicenter, Open-Label Randomized Controlled Trial. Arthritis Rheumatol (Hoboken, NJ). 2016;68: 1810-7. doi:10.1002/art.39626

25. van der Maas A, Lie E, Christensen R, Choy E, de Man YA, van Riel P, et al. Construct and criterion validity of several proposed DAS28-based rheumatoid arthritis flare criteria: an OMERACT cohort validation study. Ann Rheum Dis. 2013;72: 1800-5. doi:10.1136/annrheumdis-2012-202281

26. Prevoo ML, van 't Hof MA, Kuper $\mathrm{HH}$, van Leeuwen MA, van de Putte LB, van Riel PL. Modified Disease Activity Scores that include twenty-eight-joint counts: development and validation in a prospective longitudinal study of patients with rheumatoid arthritis. Arthritis Rheum. 1995;38: 44-48.

27. Bakker MF, Cavet G, Jacobs JW, Bijlsma JWJ, Haney DJ, Shen Y, et al. Performance of a multi-biomarker score measuring rheumatoid arthritis disease activity in the CAMERA tight control study. Ann Rheum Dis. 2012;71: 1692-7. doi:10.1136/ annrheumdis-2011-200963

28. Hirata S, Dirven L, Shen Y, Centola M, Cavet G, Lems WF, et al. A multi-biomarker score measures rheumatoid arthritis disease activity in the BeSt study. Rheumatology (Oxford). 2013;52: 1202-7. doi:10.1093/ rheumatology/kes362 


\section{SUPPLEMENTARY FILES}

Supplementary Table 1. Disease relapse by three criteria at $\mathbf{1 2}$ months for patients classified by baseline MBDA score in those patients included based on at least two available DAS28 scores $<3.2$ in the six months prior to inclusion

\begin{tabular}{lccccc}
\hline Criterion for relapse & Total & $\begin{array}{c}\text { Low (<30) } \\
\mathbf{n = 2 1 4}\end{array}$ & $\begin{array}{c}\text { Moderate (30-44) } \\
\mathbf{n = 1 4 5}\end{array}$ & $\begin{array}{c}\text { High (>44) } \\
\mathbf{n = 5 4}\end{array}$ & P \\
\hline TNFi restart & 183 & $88(47.3 \%)$ & $62(51.2 \%)$ & $33(67.3 \%)$ & 0.044 \\
Medication escalation & 214 & $101(54.3 \%)$ & $75(62.0 \%)$ & $38(77.6 \%)$ & 0.011 \\
Physician-reported flare & 204 & $98(52.7 \%)$ & $69(57.0 \%)$ & $37(75.5 \%)$ & 0.016 \\
Any criterion & 234 & $111(59.7 \%)$ & $84(69.4 \%)$ & $39(79.6 \%)$ & 0.019 \\
\hline
\end{tabular}

Any criterion $=$ TNFi re-initation, medication escalation, or physician-reported flare. P-value by Pearson $\mathrm{X}^{2}$ test. Total $\mathrm{N}=356$.

Supplementary Table 2. Disease relapse by three criteria at 12 months for patients classified by baseline MBDA score excluding 26 patients with DAS28 $\geq 3.2$ at baseline

\begin{tabular}{lccccc}
\hline Criterion for relapse & Total & $\begin{array}{c}\text { Low (<30) } \\
\mathbf{n = 2 1 4}\end{array}$ & $\begin{array}{c}\text { Moderate (30-44) } \\
\mathbf{n = 1 4 5}\end{array}$ & $\begin{array}{c}\text { High (>44) } \\
\mathbf{n = 5 4}\end{array}$ & P \\
\hline TNFi restart & 205 & $99(46.3 \%)$ & $69(47.6 \%)$ & $37(68.5 \%)$ & 0.012 \\
Medication escalation & 240 & $113(52.8 \%)$ & $86(59.3 \%)$ & $41(75.9 \%)$ & 0.008 \\
Physician-reported flare & 236 & $113(52.8 \%)$ & $82(56.6 \%)$ & $41(75.9 \%)$ & 0.009 \\
Any criterion & 269 & $127(59.3 \%)$ & $99(68.3 \%)$ & $43(79.6 \%)$ & 0.012 \\
\hline
\end{tabular}

Any criterion $=$ TNFi re-initation, medication escalation, or physician-reported flare. P-value by Pearson $x 2$ test. Total $\mathrm{N}=413$.

Supplementary Table 3. Disease relapse by three criteria at 12 months for patients classified by baseline MBDA score for patients in DAS28 remission at baseline

\begin{tabular}{lccccc}
\hline & Total & Low (<30) & Moderate (30-44) & High (>44) & P \\
Criterion for relapse & & $\mathbf{n = 2 2 0}$ & $\mathbf{n = 1 5 5}$ & $\mathbf{n = 6 4}$ & \\
\hline TNFi restart & 170 & $88(46.6 \%)$ & $55(46.2 \%)$ & $27(65.9 \%)$ & 0.065 \\
Medication escalation & 196 & $100(52.9 \%)$ & $67(56.3 \%)$ & $29(70.7 \%)$ & 0.114 \\
Physician-reported flare & 191 & $97(51.3 \%)$ & $64(53.8 \%)$ & $30(73.2 \%)$ & 0.038 \\
Any criterion & 217 & $109(57.7 \%)$ & $77(64.7 \%)$ & $31(75.6 \%)$ & 0.078 \\
\hline
\end{tabular}

Any criterion $=$ TNFi re-initation, medication escalation, or physician-reported flare. P-value by Pearson $x 2$ test. Total $\mathrm{N}=349$. 
Supplementary Table 4. Univariate associations between baseline variables and three definitions of disease relapse within 12 months in the stop group

\begin{tabular}{|c|c|c|c|c|}
\hline Characteristic & TNFi restart & $\begin{array}{l}\text { Medication } \\
\text { escalation }\end{array}$ & $\begin{array}{l}\text { Physician- } \\
\text { reported flare }\end{array}$ & Any flare \\
\hline \multirow[t]{2}{*}{ Female sex } & 0.93 (0.63-1.39); & 0.86 (0.57-1.29); & 0.95 (0.63-1.42); & 1.01 (0.66-1.53); \\
\hline & 0.735 & 0.452 & 0.799 & 0.976 \\
\hline \multirow[t]{2}{*}{ Age } & 1.00 (0.98-1.02); & 1.01 (0.99-1.03); & 1.01 (0.99-1.03); & 1.01 (0.99-1.03); \\
\hline & 0.990 & 0.242 & 0.170 & 0.300 \\
\hline \multirow[t]{2}{*}{ Disease duration } & 1.05 (1.02-1.08); & 1.04 (1.01-1.06); & 1.04 (1.01-1.06); & 1.04 (1.01-1.07); \\
\hline & $<0.001$ & 0.003 & 0.003 & 0.003 \\
\hline \multirow[t]{2}{*}{ BMI } & 1.05 (1.01-1.10); & 1.07 (1.02-1.13); & 1.04 (0.99-1.09); & 1.06 (1.01-1.12); \\
\hline & 0.030 & 0.005 & 0.137 & 0.018 \\
\hline \multirow[t]{2}{*}{ RF positive } & 1.14 (0.75-1.74); & 1.23 (0.80-1.88); & 1.04 (0.68-1.58); & 1.14 (0.73-1.77); \\
\hline & 0.530 & 0.340 & 0.865 & 0.564 \\
\hline \multirow[t]{2}{*}{ Anti-CCP positive } & 1.06 (0.70-1.62); & 1.00 (0.65-1.55); & 1.31 (0.86-1.01); & 0.99 (0.63-1.56); \\
\hline & 0.775 & 0.988 & 0.209 & 0.977 \\
\hline \multirow[t]{2}{*}{ Erosive disease } & 1.62 (1.08-2.44); & 1.46 (0.96-2.20); & 1.31 (0.87-1.97); & 1.50 (0.98-2.30); \\
\hline & 0.020 & 0.075 & 0.195 & 0.060 \\
\hline \multirow[t]{2}{*}{ DAS28-ESR } & 1.25 (0.98-1.60); & 1.57 (1.21-2.04); & 1.36 (1.06-1.75); & 1.65 (1.26-2.17); \\
\hline & 0.074 & 0.001 & 0.017 & $<0.001$ \\
\hline \multirow[t]{2}{*}{ MBDA score } & 1.02 (1.01-1.04); & 1.03 (1.02-1.05); & 1.02 (1.01-1.04); & 1.04 (1.02-1.05); \\
\hline & 0.008 & $<0.001$ & 0.002 & $<0.001$ \\
\hline \multirow[t]{2}{*}{ Number of TNFi } & 1.12 (0.71-1.76); & 1.05 (0.66-1.67); & 1.42 (0.88-2.29); & 1.31 (0.79-2.16); \\
\hline & 0.620 & 0.825 & 0.154 & 0.301 \\
\hline Concomitant & 0.83 (0.35-1.96); & 0.68 (0.29-1.61); & 1.09 (0.45-2.60); & 0.74 (0.31-1.77); \\
\hline DMARD & 0.670 & 0.381 & 0.852 & 0.495 \\
\hline
\end{tabular}

Values are for OR (95\% Cl) and P-value; boldfaced when $\mathrm{P}<0.05$. Age, disease duration, BMI, DAS28-ESR and MBDA score were analyzed as continuous variables. All others were analyzed as categorical variables. $\mathrm{N}=439$. 
Supplementary Table 5. Univariate and multivariate analyses of high (>44) versus moderate or low baseline MBDA score as a predictor of disease relapse at 12 months. Sensitivity analysis with all patients with a missing visit (missing DAS28 score at 3, 6, 9 or 12 months) counted as a flare on all flare criteria.

\begin{tabular}{|c|c|c|c|c|c|c|}
\hline \multirow[b]{2}{*}{ Criterion for relapse } & \multicolumn{2}{|c|}{ Unadjusted } & \multicolumn{2}{|l|}{ Adjusted } & \multicolumn{2}{|c|}{ Fully adjusted } \\
\hline & OR (95\% Cl) & $\mathbf{P}$ & OR (95\% Cl) & $\mathbf{P}$ & OR (95\% Cl) & $\mathbf{P}$ \\
\hline \multicolumn{7}{|l|}{ TNFi restart } \\
\hline $\mathrm{MBDA}>44$ & $2.13(1.15-3.94)$ & 0.016 & $1.90(1.02-3.55)$ & 0.044 & $1.82(0.93-3.56)$ & 0.083 \\
\hline DAS28-ESR & & & $1.32(1.01-1.72)$ & 0.040 & 1.32 (0.99-1.77) & 0.059 \\
\hline Disease duration & & & & & $1.07(1.03-1.10)$ & $<0.001$ \\
\hline $\mathrm{BMI}$ & & & & & $1.02(0.97-1.07)$ & 0.443 \\
\hline Erosive & & & & & $1.26(0.80-2.00)$ & 0.318 \\
\hline \multicolumn{7}{|l|}{ Medication escalation } \\
\hline \multicolumn{7}{|l|}{ MBDA $>44$} \\
\hline DAS28-ESR & $2.64(1.30-5.35)$ & 0.007 & $2.24(1.09-4.60)$ & 0.029 & $2.04(0.93-4.45)$ & 0.074 \\
\hline Disease duration & & & $1.52(1.14-4.60)$ & 0.004 & $1.58(1.15-2.17)$ & 0.004 \\
\hline $\mathrm{BMl}$ & & & & & 1.06 (1.03-1.10) & $<0.001$ \\
\hline \multirow[t]{2}{*}{ Erosive } & & & & & 1.04 (0.99-1.10) & 0.129 \\
\hline & & & & & 1.33 (0.81-2.17) & 0.256 \\
\hline \multicolumn{7}{|l|}{ Physician-reported } \\
\hline \multicolumn{7}{|l|}{ flare } \\
\hline MBDA $>44$ & $2.50(1.26-4.95)$ & 0.009 & $2.21(1.10-4.42)$ & 0.025 & $2.07(1.01-4.26)$ & 0.048 \\
\hline DAS28-ESR & & & 1.35 (1.03-1.79) & 0.033 & 1.32 (0.98-1.78) & 0.064 \\
\hline Disease duration & & & & & 1.05 (1.02-1.08) & 0.001 \\
\hline $\mathrm{BMI}$ & & & & & 1.00 (0.95-1.06) & 0.916 \\
\hline Erosive & & & & & $1.00(0.62-1.60)$ & 0.996 \\
\hline \multicolumn{7}{|l|}{ Any criterion } \\
\hline MBDA $>44$ & $2.62(1.21-5.68)$ & 0.015 & $2.20(1.00-4.83)$ & 0.050 & $1.88(0.83-4.26)$ & 0.131 \\
\hline DAS28-ESR & & & $1.55(1.14-2.10)$ & 0.005 & $1.54(1.11-2.14)$ & 0.011 \\
\hline Disease duration & & & & & $1.06(1.02-1.10)$ & 0.001 \\
\hline $\mathrm{BMl}$ & & & & & 1.04 (0.98-1.10) & 0.209 \\
\hline Erosive & & & & & $1.34(0.81-2.23)$ & 0.257 \\
\hline
\end{tabular}

DAS28-ESR, disease duration and BMI were analyzed as continuous variables; MBDA score ( $>44$ ) and erosive (yes/ no) were analyzed as categorical variables. Adjusted = adjusted for DAS28. Fully adjusted = adjusted for DAS28, disease duration, BMI and erosions. Any criterion includes patients with TNFi re-initation, medication escalation or physician-reported flare. Total $\mathrm{N}=439$. 
MULTI-BIOMARKER DISEASE ACTIVITY SCORE AS A PREDICTOR OF DISEASE RELAPSE IN PATIENTS WITH RHEUMATOIR ARTHRITIS STOPPING TNF INHIBATOR TREATMENT 



\section{Chapter 5}

\section{Impact of Stopping Tumor Necrosis \\ Factor-inhibitors on Rheumatoid Arthritis \\ Patients' Burden of Disease.}

Arthritis Care Res (Hoboken). 2018 Apr;70(4):516-524. doi: 10.1002/acr.23315.

Marjan Ghiti Moghadam, MD';

Peter M. ten Klooster, $\mathrm{PhD}^{2}$;

Harald E. Vonkeman, MD, $\mathrm{PhD}^{3}$;

Eva L. Kneepkens, MD, PhD4;

Ruth Klaasen, MD, PhD;

Jan N. Stolk, MD, PhD';

Ilja Tchetverikov, MD, PhD;

Simone A. Vreugdenhil, $\mathrm{MD}^{8}$;

Jan M. van Woerkom, MD, PhD';
Yvonne PM Goekoop-Ruiterman, MD, PhD ${ }^{10}$;

Robert B.M. Landewé, MD, PhD"1;

Piet L.C.M. van Riel, MD, PhD²;

Mart A.F.J. van de Laar, MD, PhD ${ }^{13}$;

Tim L. Jansen, MD, PhD ${ }^{14}$;

on behalf of the Dutch National POET

Collaboration. 


\section{ABSTRACT}

\section{OBJECTIVE}

To determine the impact of stopping tumor necrosis factor inhibitor (TNFi) treatment on patient-reported outcomes (PROs) of physical and mental health status, health utility, pain, disability and fatigue in patients with established rheumatoid arthritis (RA).

\section{METHODS}

In the pragmatic 12-month POET trial, 817 RA patients with $\geq 6$ months of remission or stable Iow disease activity were randomized 2:1 to stopping or continuing TNFi. In case of flare, TNFi was restarted at the discretion of the rheumatologist. PROs were assessed every 3 months.

\section{RESULTS}

TNFi was restarted within 12 months in 252/531 patients (47.5\%) in the stop group. At 3 months, mean PRO scores were significantly worse in the stop group and a larger proportion of patients experienced a minimal clinically important difference (MCID) on all PROs. Effect sizes (ES) were strongest for health utility $(E S=-0.24)$ and pain $(E S=-0.30)$. Mean scores improved again after this point but disability scores remained significantly different at 12 months. After 12 months, the relative risk of experiencing a MCID ranged from 1.16 for mental health status to 1.58 for fatigue. Mean PRO scores for patients restarting TNFi within 6 months were no longer significantly different from those that did not restart TNFi at 12 months.

\section{CONCLUSION}

Stopping TNFi had a significant negative short-term impact on a broad range of PROs. Long-term negative consequences appeared to be limited and outcomes in patients needing to restart TNFi within the first 6 months tended to be restored at 12 months. 
The introduction of biological agents such as tumor necrosis factor inhibitors (TNFi) has drastically improved the outcomes and clinical course of rheumatoid arthritis (RA). Their efficacy and effectiveness has been extensively demonstrated (1-3), and several trials have additionally demonstrated rapid and sustained improvements in a range of patientreported outcomes (PROs), including health-related quality of life, general health, pain, disability, and fatigue ${ }^{(4-8)}$.

As the long-term use of TNFi is costly and associated with increased risk of side effects ${ }^{(9-11)}$, the possibility of stopping TNFi in patients with stable low disease activity or remission is receiving increasing interest. Several retrospective studies have suggested that stopping TNFi is possible in some patients ${ }^{(12,13)}$, although two randomized trials showed that the majority of patients experienced a disease relapse within one year after stopping ${ }^{(14,5)}$. This finding was recently confirmed in the POET trial (16), which showed that $51.2 \%$ of patients who stopped TNFi experienced a clinical flare within 12 months of follow-up compared to 18.2\% of patients who continued TNFi treatment.

Current studies on stopping TNFi have primarily focused on the effects of discontinuation on disease activity status as the primary endpoint. However, clinical measures of disease activity may not adequately reflect the perception of illness and symptoms by RA patients themselves ${ }^{(17-20)}$. Moreover, those studies that did report one or more patient-reported outcomes (PROs) as secondary endpoints only examined group-level changes, providing no information on the consequences of stopping TNFi at the individual patient level (21). Consequently, little is currently known about the effects of trying to stop TNFi on the burden of disease as experienced by patients, especially in those patients who cannot successfully stop. The objectives of the current study were to determine the effects of stopping TNFi treatment on important PROs and to explore the course of symptoms in patients restarting TNFi after a flare.

\section{METHODS}

\section{PATIENTS}

Detailed study design and results of primary endpoints of the POET trial have been published (16). Briefly, the POET study was a pragmatic open-label trial in which RA patients with stable low disease activity (LDA), defined as 28-joint Disease Activity Score (DAS28) $<3.2$ for at least 6 months prior to inclusion, from 47 rheumatology centers throughout the Netherlands were randomized 2:1 to either stop or continue TNFi treatment. All participating patients were $\geq 18$ years old, had RA per ACR 1987 criteria, and had received TNFi treatment 
for at least one year prior to inclusion. Concomitant treatment with conventional synthetic DMARDs (csDMARDs) was continued. If RA flared, defined as a DAS28 $\geq 3.2$ with an increase $>0.6^{(22)}$, TNFi could be restarted at the discretion of the treating rheumatologist. The study was approved by the Ethical Review Boards of all participating hospitals and conducted in accordance with Good Clinical Practice guidelines and the Declaration of Helsinki. The POET study is registered in the Netherlands Trial Register, number NTR3112.

In total, 531 patients were allocated to the stop group and 286 to the continuation group and followed for 12 months ${ }^{(16)}$. The primary outcome of the study was that significantly more people in the stop group (272/531 [51.2\%]) experienced a DAS28 flare than in the continuation group (52/286 [18.2\%]). Most of these flares (213/272 [78.3\%]) occurred within 6 months after stopping TNFi. TNFi was restarted within 6 months in 211 patients (39.7\%) and within 12 months in 252 patients (47.5\%) in the stop group. Almost 85\% of the patients who restarted TNFi within the first 6 months had regained low disease activity at 12 months.

\section{MEASUREMENTS}

Patients were evaluated by their treating rheumatologist and rheumatology nurse at baseline and at least once every 3 months thereafter, for a period of one year. Baseline measures included: age, sex, weight, height, disease duration, medication use, rheumatoid factor (RF) and ACPA status, and concomitant use of csDMARDs. Clinical measurements were performed at every scheduled or unscheduled visit and included the ESR ( $\mathrm{mm} / \mathrm{h}), \mathrm{CRP}(\mathrm{mg} / \mathrm{l}), 28$-joint tender joint count (TJC28), 28-joint swollen joint count (SJC28), and a patient-reported assessment of general health on a 100-mm visual analog scale (VAS-GH). These component measures were combined to calculate the DAS28-ESR (23). Physician-reported flares and all changes in medication were continuously recorded throughout the study.

Patients additionally completed six established PROs at baseline and before every study visit, including the 36-item short-form (SF-36) health survey version 2.0 (24), five-dimensional EuroQol (EQ-5D) ${ }^{(25)}$, the Health Assessment Questionnaire Disability Index (HAQ-DI) (26), and the Bristol RA Fatigue Multidimensional Questionnaire(BRAF-MDQ) ${ }^{(27)}$. Except for the BRAF-MDQ, well-established values for minimal clinically important differences (MCIDs) are available for each of these PROs as described below. Patients in the stop group also rated their overall experience of stopping TNFi before each study visit.

\section{MEASURES}

\section{Health status}

The SF-36 assesses different aspects of health represented in 8 scales ${ }^{(24,28)}$. The eight SF36 scales are linearly transformed to range from 0 to 100 , with higher scores representing 
better health status. Additionally, the scale scores can be aggregated into a physical component summary (PCS) and a mental component summary (MCS). The component summary scores are standardized using normative data from the 1998 US general population with a mean score of 50 and a standard deviation of 10. Changes of 5 to 10 points on the 0 to100 scales of the SF-36 and 2.5 to 5 on the norm-based component scales on the scales are considered to be clinically meaningful ${ }^{(29,30)}$. The data were analyzed based on an MCID of $\geq 10$ points on the bodily pain (BP) scale and $\geq 5$ points on the component summaries as this corresponds closely with the half a standard deviation rule of thumb (31).

\section{Health utility}

Health-related quality of life was additionally measured with the EuroQol (EQ-5D) (32). The EQ-5D assesses problems in five domains (mobility, self-care, usual activities, pain/ discomfort, and anxiety/depression) on 3-point scales to produce a single, interval-level utility score. Utilities reflect the relative desirability of the health state where 0 refers to death and 1 refers to full health. Utility scores were calculated using the Dutch tariff ${ }^{(33)}$. The MCID for the EQ-5D in RA was reported to be $0.05^{(34)}$.

\section{Functional disability}

The HAQ-DI contains 20 items measuring limitations over the past week in eight categories of daily living ${ }^{(26,35)}$. Each item is scored on a 4-point rating scale from 0 (without any difficulty) to 3 (unable to do). The standard total disability score was calculated by determining the highest score in each of the eight categories, corrected for the use of aids and devices, and then averaging the category scores ${ }^{(36)}$. Scores on the HAQ-DI range between 0 and 3 , with higher values indicating more disability. A 2-step difference in score, or 0.25 units, is considered clinically significant ${ }^{(37)}$.

\section{Fatigue}

The BRAF-MDQ is a disease-specific, 20-item questionnaire covering domains of physical fatigue (4 items), living (7 items), cognition (5 items), and emotion (4 items) ${ }^{(27)}$. All 20 items can be summed to produce a global fatigue score (range 0 to 70 ), with higher scores representing worse fatigue. As no formal MCID has been established for the BRAF-MDQ, a minimal increase of 7 points was considered to indicate clinically relevant worsening, which corresponds to approximately half a standard deviation as reported in previous studies $(27,38,39)$.

\section{Patient-reported experience of stopping TNFi}

Patients in the stop group rated their experience of stopping TNFi on a single item ("I experience stopping with the TNF blocker as...") with a Likert-type response scale ranging from 1 (very positive) to 5 (very negative). 


\section{STATISTICAL ANALYSIS}

Analyses were conducted on the intent-to-treat (ITT) population which included all randomized patients. Mean scores on the PROs over time in the stop and continuation group were compared using generalized estimating equations (GEE) models with exchangeable correlation structures for repeated linear data with group (discontinuation vs. continuation), time $(0,3,6$, and 12 months), and group*time interaction as categorical factors to detect any difference in PRO trajectories over time. Post-hoc t-tests were performed to analyze between-group differences in scores at the different time points. Based on estimated marginal means and corresponding standard errors from the GEE models, between-group standardized effect sizes (ES) were expressed as Cohen's d ( $\triangle$ estimated marginal mean / pooled standard deviation) with $95 \%$ confidence intervals $(95 \% \mathrm{Cl}$ ) where 0.20 denotes a small, 0.50 a moderate, and 0.80 a large effect ${ }^{(40)}$. Besides comparing mean scores on a group level, individual patient-level analyses were performed by calculating the proportion of patients experiencing a clinically important worsening of symptoms on each PRO at each time point as compared to baseline. Proportions of patients experiencing a worsening of symptoms $\geq \mathrm{MCID}$ were compared between the stop and continuation group using binary logistic GEE models, again with group, time (3, 6, and 12 months), and group*time as factors. Post-hoc chi-square tests and relative risks (RR) with $95 \% \mathrm{Cl}$ were used to examine the significance and magnitude of differences at each follow-up point. Additional descriptive analyses (linear GEE models without adjustment for covariates) were performed in the stop group only to compare Iongitudinal scores on each PRO between patients who needed to restart TNFi within 6 months versus those that did not restart during the full 12 months of the study.

\section{RESULTS}

\section{PATIENT CHARACTERISTICS AND BASELINE SCORES}

Baseline demographics, disease characteristics and PRO scores were similar in both groups (Table 1). Most patients had longstanding, RF positive, disease. The majority (85.9\%) was on their first TNFi, predominantly adalimumab or etanercept. Scores on all PROs indicated relatively mild disease impact at baseline, as would be expected for RA patients in stable remission or low disease activity. Mean SF-36 component summary scores indicated that patients' mental health was comparable to general population norms, while their physical health score was approximately half a standard deviation below the population mean. PRO scores were significantly but modestly correlated with DAS28-ESR scores over the different time points (Pearson r's ranging from 0.08 to 0.45 ), confirming that patient-perceived symptoms and clinical disease activity reflected related but distinct 
aspects of the disease (Supplementary Table 1).

\section{Table 1. Baseline characteristics of the patients}

\begin{tabular}{|c|c|c|}
\hline Characteristic & $\begin{array}{l}\text { Stop TNFi } \\
(n=531)\end{array}$ & $\begin{array}{l}\text { Continue TNF } \\
(n=286)\end{array}$ \\
\hline Female, $\mathrm{n}(\%)$ & $362(68.2)$ & $188(66.0)$ \\
\hline Age, mean $(S D)$ years & $60.0(11.8)$ & $59.7(10.6)$ \\
\hline Disease duration, mean (SD) years & $12.0(8.8)$ & $11.1(8.4)$ \\
\hline DAS28, mean (SD) & $1.97(0.76)$ & $2.05(0.74)$ \\
\hline RF positive, n (\%) & $328(67.5)$ & $178(67.4)$ \\
\hline \multicolumn{3}{|l|}{ TNFi, n (\%) } \\
\hline Adalimumab & $271(51.1)$ & $129(45.1)$ \\
\hline Etanercept & $213(40.2)$ & $133(46.5)$ \\
\hline Infliximab & $25(4.7)$ & $14(4.9)$ \\
\hline Golimumab & $15(2.8)$ & $8(2.8)$ \\
\hline Certolizumab & $6(1.1)$ & $2(0.7)$ \\
\hline \multicolumn{3}{|l|}{ Number of TNFi taken, n (\%) } \\
\hline $1^{\mathrm{st}}$ & $459(86.6)$ & $243(85.0)$ \\
\hline $2^{\text {nd }}$ & $61(11.5)$ & $37(12.9)$ \\
\hline $3^{\text {rd }}$ & $10(1.9)$ & $6(2.1)$ \\
\hline \multicolumn{3}{|l|}{ SF-36, mean (SD) } \\
\hline Physical component summary score & $45.6(8.8)$ & $45.3(8.8)$ \\
\hline Mental component summary score & $52.0(8.9)$ & $51.64(10.2)$ \\
\hline Bodily pain score & 71.9 (19.4) & $72.1(19.3)$ \\
\hline EQ-5D, mean (SD) & $0.84(0.18)$ & $0.85(0.14)$ \\
\hline HAQ-DI, mean (SD) & $0.60(0.62)$ & $0.59(0.59)$ \\
\hline BRAF-MDQ, mean (SD) & $14.7(11.2)$ & $15.4(12.2)$ \\
\hline
\end{tabular}

BRAF-MDQ = Bristol RA Fatigue Multidimensional Questionnaire;DAS28 = disease activity score in 28 joints; EQ-5D = five-dimensional EuroQol; HAQ-DI = Health Assessment Questionnaire Disability Index; RF = rheumatoid factor; SF-36 = 36-item short-form health survey; TNFi = tumor necrosis factor-alpha inhibitor.

\section{PRO SCORES IN PATIENTS STOPPING VS. CONTINUING TNFi}

The GEE analyses (Supplementary Table 2) showed a significant group by time interaction for all PROs, except for the EQ-5D, indicating that mean scores changed significantly different between both groups over time. Although all effect sizes were of small magnitude, post-hoc t-tests confirmed that mean scores at 3 months were significantly worse in the stop group than those in the continuation group on all PROs (Figures 1-3). Mean SF-36 MCS appeared to improve slightly between baseline and 3-month follow-up in the continuation group, but this improvement was not significant (paired t-test, $P=0.192$ ). 
The short-term impact of stopping TNFi was largest for pain with SF-36 BP scores being around 7 points lower $(E S=-0.30$ ) in the stop group at 3 months. After this point, PRO scores in the stop group tended to stabilize and steadily improve again. At 6 months, physical health status, pain and functional disability scores remained significantly worse in the stop group. Pain in the stop group improved further and was no longer significantly different at 12 months. Functional disability scores, however, remained slightly $(E S=0.18)$ but significantly higher in the stop group.

\section{MCIDs IN PATIENTS STOPPING VS. CONTINUING TNFi}

The proportion of patients experiencing a worsening $\geq \mathrm{MCID}$ at 3 months was significantly larger in the stop group for all PROs (Supplementary Table 3 and Figures 1-3). Except for the SF-36 MCS, there was no interaction between group and time, indicating that this difference between groups remained stable over the remaining course of follow-up. A substantial number of patients continuing TNFi also experienced a MCID due to natural fluctuations or measurement error. The proportion of patients experiencing a MCID was largest for pain at all follow-up time points in both the continuation (31.3\% to 64.0\%) and the stop group (49.5\% to 80.0\%). The RR for experiencing a MCID at 3 months ranged from 1.63 on the EQ-5D to 2.19 on the SF-36 MCS for patients stopping vs. continuing TNFi. Over the course of follow-up RRs decreased for al PROs, but the differences remained significant for all PROs except mental health status (Figures 1-3). After 12 months, trying to stop TNFi had resulted in an additional 5.5\% (SF-36 MCS) to 18.6\% (HAQ-DI) of patients experiencing a clinically important worsening on top of those observed in the continuation group.

\section{PRO SCORES IN PATIENTS RESTARTING VS. NOT RESTARTING TNFi}

At 6 months, TNFi was restarted in 211 patients in the stop group (39.7\%), whereas 279 patients (52.5\%) did not restart TNFi during the entire 12 months of follow-up. Estimated mean scores in both subgroups are shown in Figures 4-5. In those patients experiencing a flare and needing to restart TNFi within the first 6 months after baseline, this was accompanied by a sharp worsening of physical health status, pain, health utility and disability scores at 3 and 6 months. Effect sizes were -0.42 (95\% Cl: -0.60 to -0.24$)$ and -0.42 (95\% Cl: -0.60 to -0.24$)$ for SF-36 PCS, -0.56 (95\% Cl: -0.74 to -0.37$)$ and -0.41 (95\% Cl: -0.59 to -0.22$)$ for SF-36 BP, -0.36 (95\% Cl: -0.54 to -0.18$)$ and -0.21 (95\% Cl: -0.39 to -0.03 ) for $\mathrm{EQ}-5 \mathrm{D}$, and 0.30 (95\% Cl: 0.12 to 0.48 ) and 0.29 (95\% Cl: 0.11 to 0.47 ) for HAQ-DI scores at 3 and 6 months, respectively. PRO scores improved rapidly after restart and were no longer significantly different from those that did not restart TNFi at all after 9 months.

\section{PATIENT-REPORTED EXPERIENCE OF STOPPING TNFi}

The proportion of patients who rated their experience of stopping TNFi as positive or very 
positive after 12 months was 38.8\%, while 43.6\% experienced stopping as negative or very negative (Supplementary Table 4). At each time point, experience ratings were strongly associated with having restarted TNFi at that time or not. Across study visits, $72.4 \%$ to $76.2 \%$ of the patients that restarted TNFi rated their experience as negative as opposed to $12.1 \%$ to $18.9 \%$ of the patients that did not restart (Supplementary Table 5).
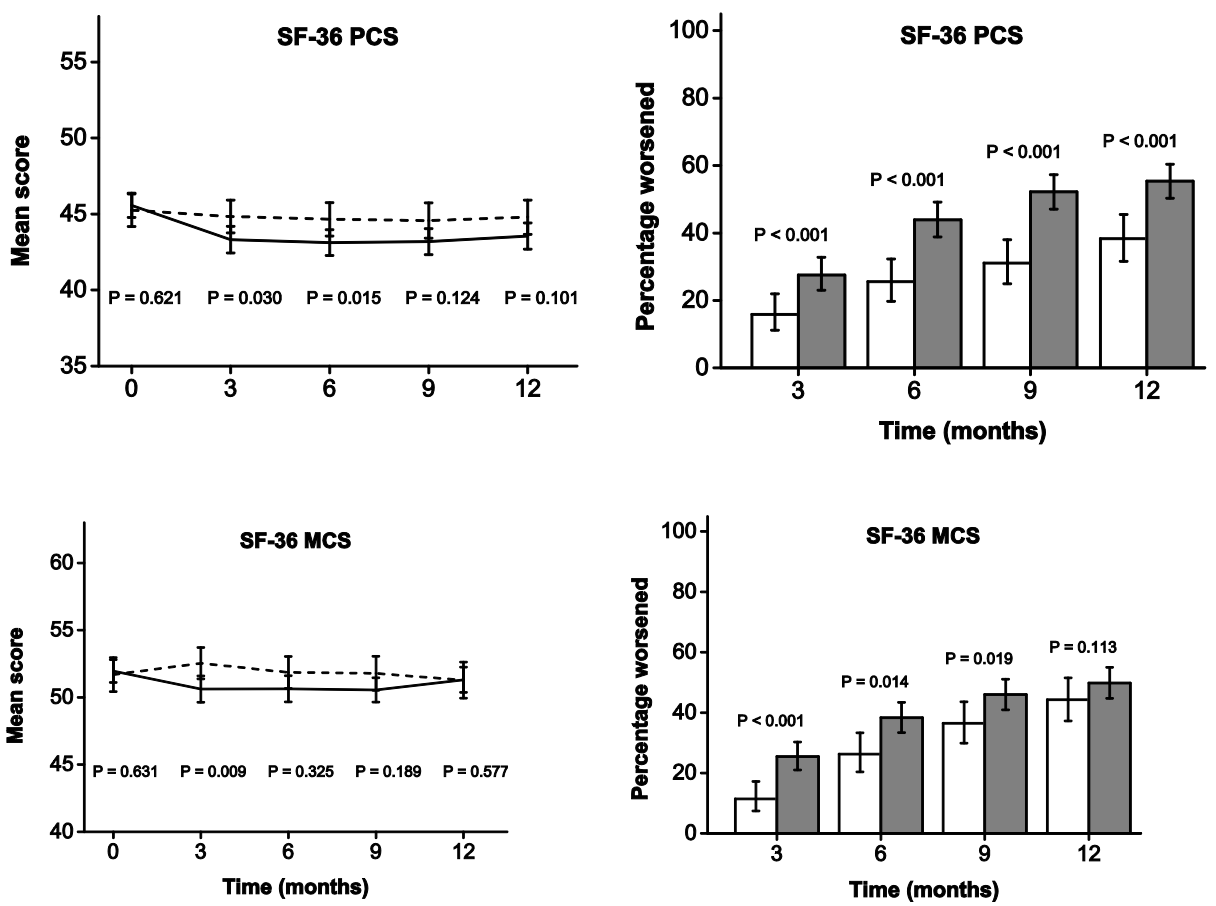

Figure 1. Estimated mean PRO scores on physical and mental health status in all patients over time (left panel), stratified into those who continued (dashed line) and stopped TNFi (solid line). Cumulative proportion of patients experiencing a MCID as compared with baseline (right panel), gray bars are patients in the stop group. Error bars are 95\% Wald Cls for both mean scores and proportions.

SF-36 = 36-item short-form health survey; PCS = physical component summary; MCS = mental component summary. ${ }^{*} P<0.05 ;{ }^{* *} P<0.01 ;{ }^{* * *} P<0.001$ for between-group t-tests or chi-square tests. 

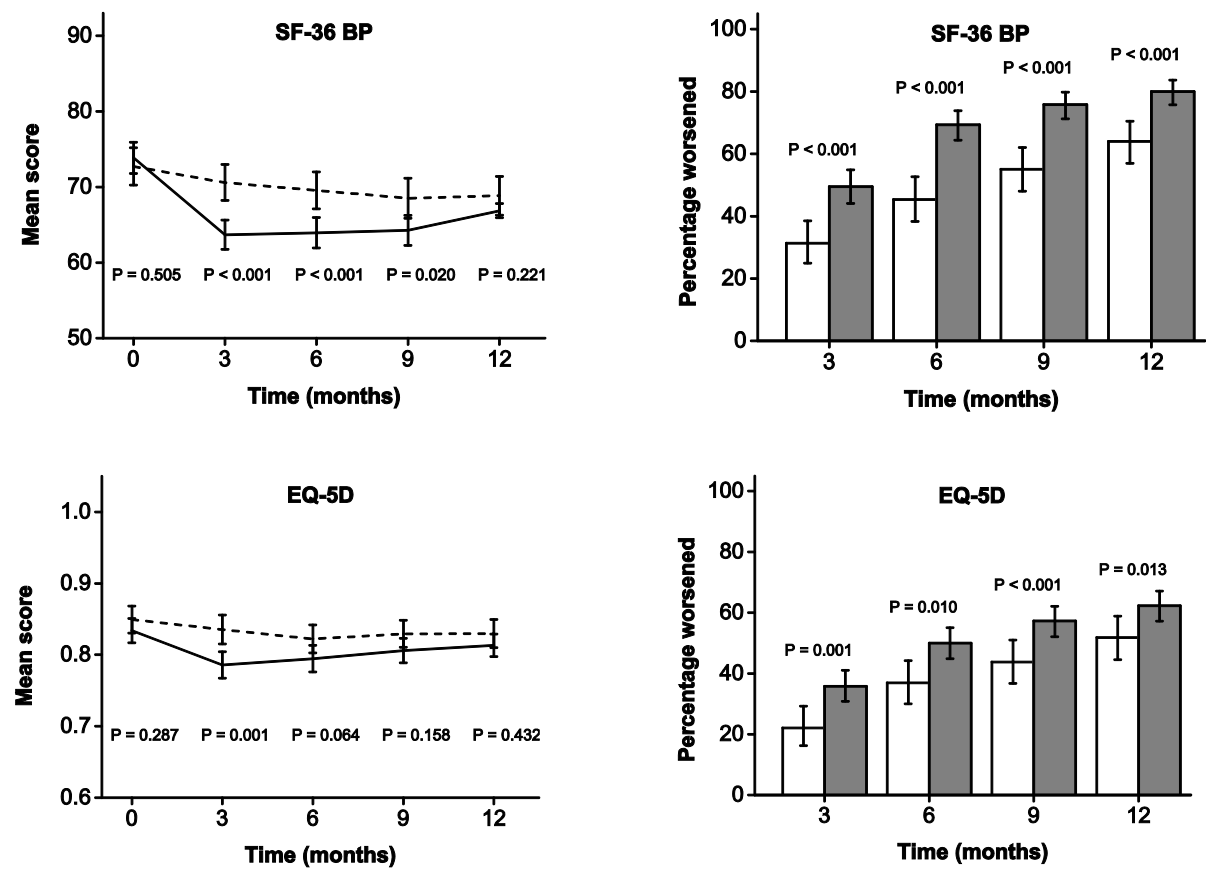

Figure 2. Estimated mean PRO scores on pain and health utility in all patients over time (left panel), stratified into those who continued (dashed line) and stopped TNFi (solid line). Cumulative proportion of patients experiencing a MCID as compared with baseline (right panel), gray bars are patients in the stop group. Error bars are $95 \%$ Wald confidence intervals for both mean scores and proportions.

P-values are for between-group t-tests or chi-square tests. SF-36 = 36-item short-form health survey; $\mathrm{BP}$ = bodily pain; $\mathrm{EQ}-5 \mathrm{D}$ = five-dimensional EuroQol. 

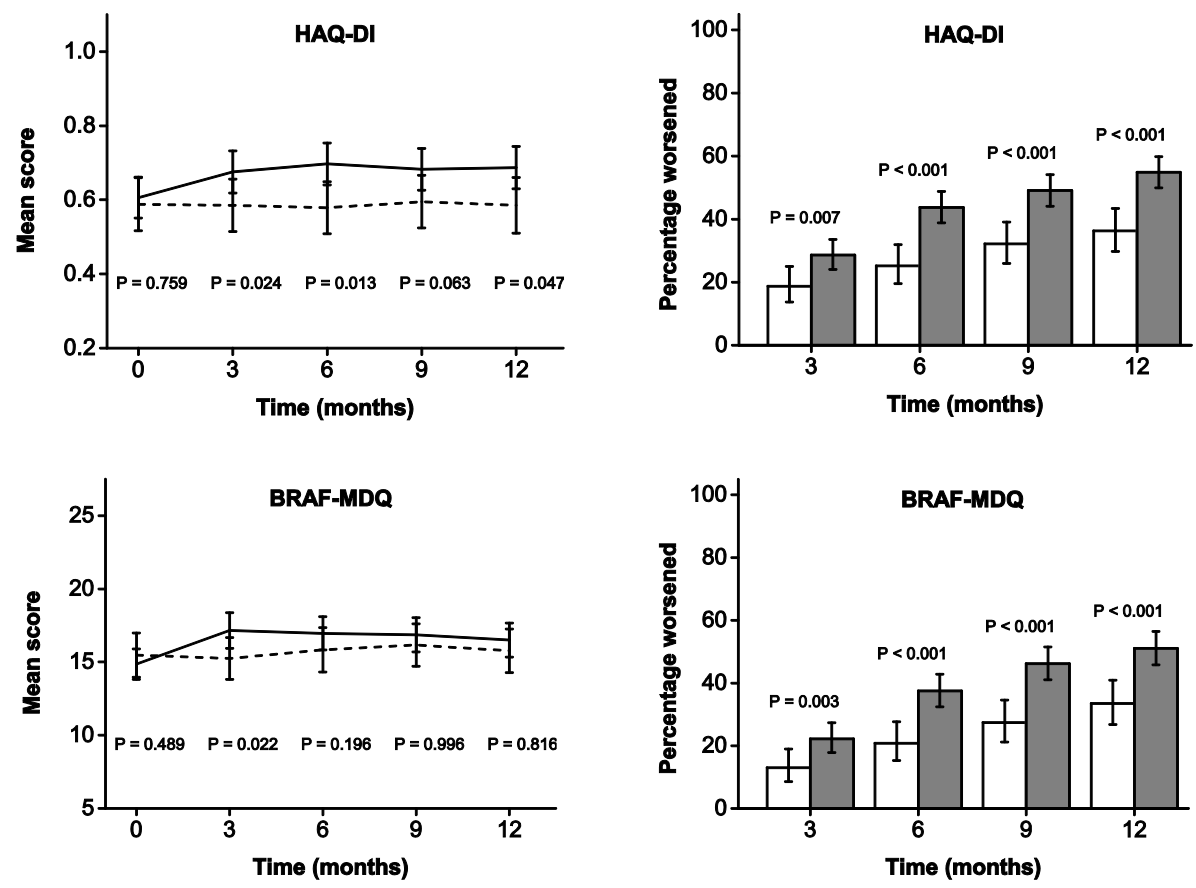

Figure 3. Estimated mean PRO scores on disability and fatigue in all patients over time (left panel), stratified into those who continued (dashed line) and stopped TNFi (solid line). Cumulative proportion of patients experiencing a MCID as compared with baseline (right panel), gray bars are patients in the stop group. Error bars are $95 \%$ Wald confidence intervals for both mean scores and proportions.

P-values are for between-group t-tests or chi-square tests. HAQ-DI = Health Assessment Questionnaire Disability Index; BRAF-MDQ = Bristol RA Fatigue Multidimensional Questionnaire. ${ }^{*} P<0.05$; ${ }^{* *} P<0.01$; ${ }^{* * *} P<0.001$ for between-group t-tests or chi-square tests. 

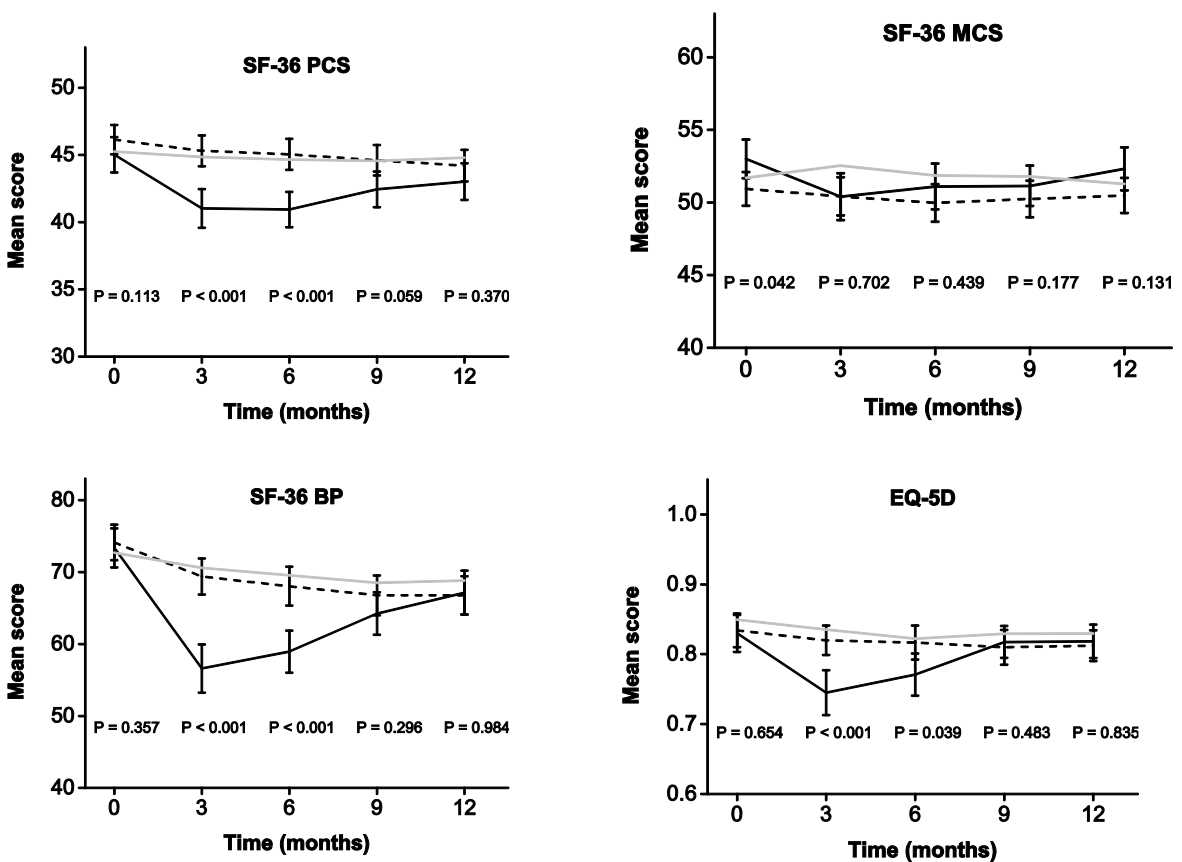

Figure 4. Estimated mean PRO scores on physical and mental health status, pain, and health utility of patients stopping TNFI, stratified into those who restarted within 6 months (solid line; $\mathbf{n}$ $=211$ ) and remained off drug throughout the 12-month study period (dashed line; $n=279$ ). Those who restarted after the 6-month visit were excluded. For comparison, mean scores in the total continuation group are plotted as solid grey lines (without error bars). Error bars are 95\% Wald confidence intervals.

P-values are for between-group t-tests or chi-square tests. SF-36 = 36-item short-form health survey version; PCS = physical component summary; MCS = mental component summary; BP = bodily pain; $E Q-5 D=$ five-dimensional EuroQol. 

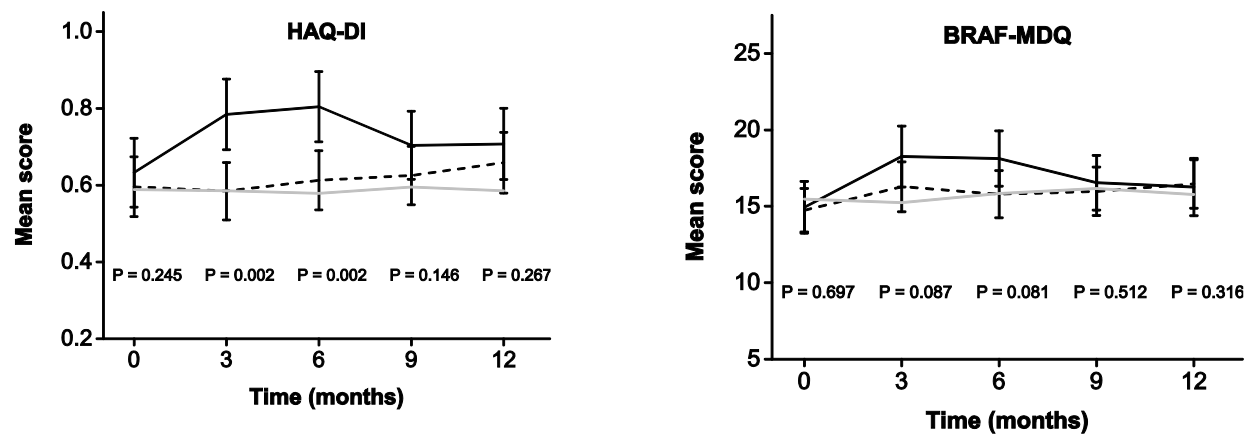

Figure 5. Estimated mean PRO scores on disability and fatigue of patients stopping TNFI, stratified into those who restarted within 6 months (solid line; $n=211$ ) and remained off drug throughout the 12-month study period (dashed line; $n=279$ ). Those who restarted after the 6-month visit were excluded. For comparison, mean scores in the total continuation group are plotted as solid grey lines (without error bars). Error bars are 95\% Wald confidence intervals.

P-values are for between-group t-tests or chi-square tests. HAQ-DI = Health Assessment Questionnaire Disability Index; BRAF-MDQ = Bristol RA Fatigue Multidimensional Questionnaire.

\section{DISCUSSION}

The POET trial showed that stopping TNFi treatment in patients with established RA in remission or with stable low disease activity resulted in substantially more clinical flares, but that most patients who restarted TNFi treatment quickly regained remission or low disease activity ${ }^{(16)}$. The current study extends these findings by demonstrating that stopping TNFi also had a significant, but small, short-term negative impact on patient-reported physical and mental health status, health utility, pain, disability and fatigue. Except for physical disability, PRO scores did not remain significantly different at 12 months. However, the proportion of patients with worsening above MCID was higher and differed significantly for more PROs (Supplementary Table 2).Moreover, subgroup analyses showed that for patients who restarted TNFi after a flare within the first 6 months, 9- and 12-month PRO scores were no longer different from those that did not restart at all. As such, a strategy of trying to stop TNFi treatment and quickly restarting in case of flare, does not appear to have substantial long-term consequences for the burden of disease as experienced by patients.

To date, very few studies have thoroughly examined the longitudinal impact of stopping 
TNFi on patient-reported health-status and symptoms. The double-blind PRESERVE study showed that after 52 weeks patients maintaining 50 mg etanercept plus methotrexate treatment had significantly better scores than patients using placebo plus methotrexate on general health, pain, disability, health utility, sleep, and fatigue ${ }^{(14)}$. Some other, nonrandomized or observational, studies specifically reported the effects of stopping TNFi on one or more PROs, usually by comparing long-term disability scores in patients who did and did not successfully discontinue TNFi. The observational RRR study showed that one-year mean HAQ-DI scores were significantly lower in patients who remained in DAS remission after stopping infliximab versus those that did not ${ }^{\left({ }^{41}\right)}$. Post-hoc analyses of 104 patients in the BeSt study who discontinued infliximab after achieving stable low disease activity showed that HAQ-DI scores at 1 and 3 years after discontinuation were similar to scores at discontinuation in both restarters and patients with sustained low disease activity (42). However, 5 years after discontinuation median disability scores were significantly increased in restarters. The open-label non-randomized HONOR study did not show a significant difference in one-year HAQ-DI scores between patients that did and did not sustain remission after adalimumab discontinuation ${ }^{(43)}$. Another very small observational study in 21 RA patients did not find significant differences in one-year HAQ-DI scores either ${ }^{(44)}$, although with only 21 analyzed patients this study was likely underpowered for this purpose.

The POET study is the first large pragmatic trial examining the impact of trying to stop TNFi on both clinical and patient-reported outcomes in realistic clinical settings. Overall, the current findings show that stopping TNFi resulted in significantly worse scores on all patient-reported outcome domains, particularly reflecting pain. However, the magnitude of differences was generally small and decreased again after 3-6 months. The proportion of patients experiencing a worsening $\geq$ the MCID within 3 months was also significantly larger in the stop group and remained significantly different at 12 months for most outcomes. At 12 months, the proportion of patients in the stop group that experienced an MCID was $>50 \%$ on all PROs except mental health status. However, a substantial proportion of patients who continued TNFi also experienced an MCID, due to either natural disease fluctuations or measurement error. Like the effect sizes for mean scores, relative risks for experiencing an MCID steadily decreased for all PROs over time.

In interpreting these findings, it is important to keep in mind that POET was an open label pragmatic trial in which, after randomization, rheumatologists were free to prescribe and adjust medications as considered clinically necessary. Consequently, it is not possible to attribute the differences between groups purely to the effects of stopping TNFi and the impact on PRO scores may be substantially confounded by other treatment decisions. For 
instance, rheumatologists are likely to have started or increased csDMARDs or NSAIDs in patients in the stop group with an increase in disease activity but not meeting the DAS28 flare criterion, resulting in an underestimation of impact. Moreover, as the follow-up period of POET was limited to one year, it is unclear how many patients experienced a disease relapse after this period. Finally, the POET study only examined the effects of completely stopping TNFi. Recently, several studies have suggested that TNFi dose reduction may be more effective in maintaining low disease activity ${ }^{(14,15)}$. This may also translate into less impact on patient-reported symptoms. For instance, a recent trial on disease activity guided tapering of adalimumab or etanercept showed that HAQ-DI and EQ-5D scores remained stable in the dose reduction group and did not differ significantly over time from patients continuing $\mathrm{TNFi}^{(45)}$.

An important strength of the current study is that it included a broad range of PROs, covering all patient-reported outcome domains as defined by the ACR and WHO/ILAR core set endpoints for RA clinical trials ${ }^{(46,47)}$, as well as fatigue, which was more recently endorsed as a symptom important to patients ${ }^{(48)}$. Moreover, well established and validated measures were used for all domains. For instance, we used the multi-item BRAF-MDQ to measure fatigue, which has better content validity and measurement precision than more commonly used single-item measures of fatigue ${ }^{(39)}$.

In summary, a strategy of trying to stop TNFi treatment in patients with stable low disease activity in daily clinical practice has a negative short-term impact on patient-perceived symptoms but does not appear to have substantial longer-term consequences for the burden of disease as experienced by patients. 


\section{REFERENCES}

1. Hochberg MC, Tracy JK, Hawkins-Holt M, Flores $\mathrm{RH}$. Comparison of the efficacy of the tumour necrosis factor alpha blocking agents adalimumab, etanercept, and infliximab when added to methotrexate in patients with active rheumatoid arthritis. Ann Rheum Dis 2003;62 Suppl 2:ii13-6.

2. Chen Y-F, Jobanputra P, Barton P, Jowett S, Bryan S, Clark W, et al. A systematic review of the effectiveness of adalimumab, etanercept and infliximab for the treatment of rheumatoid arthritis in adults and an economic evaluation of their costeffectiveness. Health Technol Assess 2006;10:iii-iv, xi-xiii, 1-229.

3. Alonso-Ruiz A, Pijoan JI, Ansuategui E, Urkaregi A, Calabozo M, Quintana A. Tumor necrosis factor alpha drugs in rheumatoid arthritis: systematic review and metaanalysis of efficacy and safety. BMC Musculoskelet Disord 2008;9:52.

4. Maini RN, Breedveld FC, Kalden JR, Smolen JS, Furst D, Weisman $\mathrm{MH}$, et al. Sustained improvement over two years in physical function, structural damage, and signs and symptoms among patients with rheumatoid arthritis treated with infliximab and methotrexate. Arthritis Rheum 2004;50:1051-65.

5. Kimel M, Cifaldi M, Chen N, Revicki D. Adalimumab plus methotrexate improved SF-36 scores and reduced the effect of rheumatoid arthritis (RA) on work activity for patients with early RA. J Rheumatol 2008;35:206-15.

6. Kekow J, Moots RJ, Emery P, Durez P,
Koenig A, Singh A, et al. Patient-reported outcomes improve with etanercept plus methotrexate in active early rheumatoid arthritis and the improvement is strongly associated with remission: the COMET trial. Ann Rheum Dis 2010;69:222-5.

7. Strand V, Smolen JS, Vollenhoven RF van, Mease P, Burmester GR, Hiepe F, et al. Certolizumab pegol plus methotrexate provides broad relief from the burden of rheumatoid arthritis: analysis of patientreported outcomes from the RAPID 2 trial. Ann Rheum Dis 2011;70:996-1002.

8. Genovese MC, Han C, Keystone EC, Hsia EC, Buchanan J, Gathany T, et al. Effect of golimumab on patient-reported outcomes in rheumatoid arthritis: results from the GO-FORWARD study. J Rheumatol 2012;39:1185-91.

9. Dreyer L, Mellemkjær L, Andersen AR, Bennett P, Poulsen UE, Juulsgaard Ellingsen $\mathrm{T}$, et al. Incidences of overall and site specific cancers in TNFa inhibitor treated patients with rheumatoid arthritis and other arthritides - a follow-up study from the DANBIO Registry. Ann Rheum Dis 2013;72:79-82.

10. Raaschou P, Simard JF, Holmqvist $M$, Askling J. Rheumatoid arthritis, antitumour necrosis factor therapy, and risk of malignant melanoma: nationwide population based prospective cohort study from Sweden. BMJ 2013;346:f1939.

11. Ramiro S, Gaujoux-Viala C, Nam JL, Smolen JS, Buch M, Gossec L, et al. Safety of synthetic and biological 
DMARDs: a systematic literature review informing the 2013 update of the EULAR recommendations for management of rheumatoid arthritis. Ann Rheum Dis 2014;73:529-35.

12. Tanaka $Y$. Intensive treatment and treatment holiday of TNF-inhibitors in rheumatoid arthritis. Curr Opin Rheumatol 2012;24:319-26.

13. Ingen ILA van, Lamers-Karnebeek F, Jansen TL. Optimizing the expediency of TNFi in rheumatoid arthritis: offering a TNFi holiday in patients having reached lowdisease activity in the maintenance phase. Expert Opin Biol Ther 2014;14:1761-7.

14. Smolen JS, Nash P, Durez P, Hall S, Ilivanova E, Irazoque-Palazuelos $F$, et al. Maintenance, reduction, or withdrawal of etanercept after treatment with etanercept and methotrexate in patients with moderate rheumatoid arthritis (PRESERVE): a randomised controlled trial. Lancet 2013;381:918-29.

15. Vollenhoven RF van, Ostergaard $M$, Leirisalo-Repo M, Uhlig $T$, Jansson $M$, Larsson E, et al. Full dose, reduced dose or discontinuation of etanercept in rheumatoid arthritis. Ann Rheum Dis 2015.

16. Ghiti Moghadam M, Vonkeman HE, Klooster PM Ten, Tekstra J, Schaardenburg D van, Starmans-Kool M, et al. Stopping Tumor Necrosis Factor Inhibitor Treatment in Patients With Established Rheumatoid Arthritis in Remission or With Stable Low Disease Activity: A Pragmatic Multicenter, Open-Label Randomized Controlled
Trial. Arthritis Rheumatol (Hoboken, NJ) 2016;68:1810-7.

17. 17. Hagglund KJ, Haley WE, Reveille JD, Alarcón GS. Predicting individual differences in pain and functional impairment among patients with rheumatoid arthritis. Arthritis Rheum 1989;32:851-8.

18. Sanderson $\mathrm{T}$, Morris M, Calnan M, Richards P, Hewlett S. Patient perspective of measuring treatment efficacy: the rheumatoid arthritis patient priorities for pharmacologic interventions outcomes. Arthritis Care Res (Hoboken) 2010;62:64756.

19. Studenic P, Radner H, Smolen JS, Aletaha D. Discrepancies between patients and physicians in their perceptions of rheumatoid arthritis disease activity. Arthritis Rheum 2012;64:2814-23.

20. Oude Voshaar AH, Taal E, Klooster PM ten, Vonkeman HE, Laar MAFJ van de. Predictors of no improvement in subjective health perception in newly diagnosed rheumatoid athritis patients with a good DAS28 response at 12 months. Arthritis Rheum 2013;65:S921.

21. Kvien TK, Heiberg T, Hagen KB. Minimal clinically important improvement/ difference (MCII/MCID) and patient acceptable symptom state (PASS): what do these concepts mean? Ann Rheum Dis 2007;66:iii40-iii41.

22. Maas A van der, Lie E, Christensen R, Choy E, Man YA de, Riel P van, et al. Construct and criterion validity of several proposed DAS28-based rheumatoid arthritis flare 
criteria: an OMERACT cohort validation study. Ann Rheum Dis 2013;72:1800-5.

23. Prevoo ML, 't Hof MA van, Kuper $\mathrm{HH}$, Leeuwen MA van, Putte LB van de, Riel PL van. Modified Disease Activity Scores that include twenty-eight-joint counts: development and validation in a prospective longitudinal study of patients with rheumatoid arthritis. Arthritis Rheum 1995;38:44-48.

24. Ware Jr. JE. SF-36 health survey update. Spine (Phila Pa 1976) 2000;25:3130-3139.

25. The EuroQol Group. EuroQol--a new facility for the measurement of health-related quality of life. Health Policy 1990;16:199-208.

26. Fries JF, Spitz P, Kraines RG, Holman HR. Measurement of patient outcome in arthritis. Arthritis Rheum 1980;23:137-145.

27. Nicklin J, Cramp F, Kirwan J, Greenwood R, Urban M, Hewlett S. Measuring fatigue in rheumatoid arthritis: a cross-sectional study to evaluate the Bristol Rheumatoid Arthritis Fatigue Multi-Dimensional questionnaire, visual analog scales, and numerical rating scales. Arthritis Care Res (Hoboken) 2010;62:1559-68.

28. Klooster PM ten, Vonkeman HE, Taal E, Siemons L, Hendriks L, Jong AJL de, et al. Performance of the Dutch SF-36 version 2 as a measure of health-related quality of life in patients with rheumatoid arthritis. Heal Qual Life Outcomes 2013;11:77.

29. Ware JE, Kosinski M, Dewey JE, Kosinski M, Dewey JE. How to score version 2 of the SF-36 health survey (standard \& acute forms). Lincoln, RI: QualityMetric Inc
Lincoln, Rl; 2000.

30. Kosinski M, Zhao SZ, Dedhiya S, Osterhaus JT, Ware Jr. JE. Determining minimally important changes in generic and disease-specific health-related quality of life questionnaires in clinical trials of rheumatoid arthritis. Arthritis Rheum 2000;43:1478-87.

31. Norman G, Sloan J, Wyrwich K. Interpretation of changes in health-related quality of life: the remarkable universality of half a standard deviation. Med Care 2003;41:582-592.

32. Brooks R. EuroQol: the current state of play. Health Policy 1996;37:53-72.

33. Lamers LM, McDonnell J, Stalmeier PFM, Krabbe PFM, Busschbach JJ V. The Dutch tariff: results and arguments for an effective design for national EQ-5D valuation studies. Health Econ 2006;15:1121-32.

34. Marra CA, Woolcott JC, Kopec JA, Shojania K, Offer R, Brazier JE, et al. A comparison of generic, indirect utility measures diseasespecific instruments (the RAQoL and the $\mathrm{HAQ}$ ) in rheumatoid arthritis. Soc Sci Med 2005;60:1571-1582.

35. Peter M, Klooster PM ten, Taal E, Laar MA van de. Rasch analysis of the Dutch Health Assessment Questionnaire disability index and the Health Assessment Questionnaire II in patients with rheumatoid arthritis. Arthritis Rheum 2008;59:1721-1728.

36. Bruce B, Fries JF. The Stanford Health Assessment Questionnaire: a review of its history, issues, progress, and documentation. J Rheumato/ 2003;30:167-178. 
37. Wolfe F, Pincus T. Listening to the patient: a practical guide to self-report questionnaires in clinical care. Arthritis Rheum 1999;42:1797-808.

38. Dures EK, Hewlett SE, Cramp F a, Greenwood R, Nicklin JK, Urban M, et al. Reliability and sensitivity to change of the Bristol Rheumatoid Arthritis Fatigue scales. Rheumatology (Oxford) 2013;52:1832-9.

39. Oude Voshaar MAH, Klooster PM Ten, Bode C, Vonkeman HE, Glas CAW, Jansen $\mathrm{T}$, et al. Assessment of fatigue in rheumatoid arthritis: a psychometric comparison of single-item, multiitem, and multidimensional measures. J Rheumatol 2015;42:413-20.

40. Cohen J. Statistical power analysis for the behavioural sciences. 2nd ed. Hillsdale, NJ: Lawrence Erlbaum Associates; 1988.

41. Tanaka Y, Takeuchi T, Mimori T, Saito K, Nawata M, Kameda $H$, et al. Discontinuation of infliximab after attaining low disease activity in patients with rheumatoid arthritis: RRR (remission induction by Remicade in RA) study. Ann Rheum Dis 2010;69:1286-91.

42. Broek M van den, Klarenbeek NB, Dirven L, Schaardenburg D van, Hulsmans HMJ, Kerstens PJSM, et al. Discontinuation of infliximab and potential predictors of persistent low disease activity in patients with early rheumatoid arthritis and disease activity score-steered therapy: subanalysis of the BeSt study. Ann Rheum Dis 2011;70:1389-94.

43. Tanaka Y, Hirata S, Kubo S, Fukuyo S, Hanami K, Sawamukai N, et al. Discontinuation of adalimumab after achieving remission in patients with established rheumatoid arthritis: 1-year outcome of the HONOR study. Ann Rheum Dis 2015;74:389-95.

44. Brocq O, Millasseau E, Albert C, Grisot C, Flory P, Roux C-H, et al. Effect of discontinuing TNFalpha antagonist therapy in patients with remission of rheumatoid arthritis. Joint Bone Spine 2009;76:350-5.

45. Herwaarden N van, Maas A van der, Minten MJM, Hoogen FHJ van den, Kievit W, Vollenhoven RF van, et al. Disease activity guided dose reduction and withdrawal of adalimumab or etanercept compared with usual care in rheumatoid arthritis: open label, randomised controlled, noninferiority trial. BMJ 2015;350:h1389.

46. Felson DT, Anderson JJ, Boers M, Bombardier C, Chernoff M, Fried B, et al. The American College of Rheumatology preliminary core set of disease activity measures for rheumatoid arthritis clinical trials. Arthritis Rheum 1993;36:729-40.

47. Boers M, Tugwell P, Felson DT, Riel PL van, Kirwan JR, Edmonds JP, et al. World Health Organization and International League of Associations for Rheumatology core endpoints for symptom modifying antirheumatic drugs in rheumatoid arthritis clinical trials. J Rheumato/ 1994;21:86-9.

48. Kirwan JR, Minnock P, Adebajo A, Bresnihan B, Choy E, Wit M de, et al. Patient perspective: fatigue as a recommended patient centered outcome measure in rheumatoid arthritis. I Rheumatol 2007;34:1174-1177. 


\section{SUPPLEMENTARY FILES}

Supplementary Table 1. Pearson correlations between DAS28-ESR scores and PRO scores in the total sample over time

\begin{tabular}{llllll}
\hline & Baseline & 3 months & 6 months & 9 months & 12 months \\
\hline SF-36 PCS & $-0.22^{* * *}$ & $-0.38^{* * *}$ & $-0.40^{* * *}$ & $-0.37^{* * *}$ & $-0.36^{* * *}$ \\
SF-36 MCS & $-0.08^{*}$ & $-0.19^{* * *}$ & $-0.10^{*}$ & -0.08 & $-0.10^{*}$ \\
SF-36 BP & $-0.25^{* * *}$ & $-0.45^{* * *}$ & $-0.44^{* * *}$ & $-0.41^{* * *}$ & $-0.40^{* * *}$ \\
EQ-5D & $-0.18^{* * *}$ & $-0.34^{* * *}$ & $-0.34^{* * *}$ & $-0.28^{* * *}$ & $-0.26^{* * *}$ \\
HAQ-DI & $0.25^{* * *}$ & $0.34^{* * *}$ & $0.34^{* * *}$ & $0.30^{* * *}$ & $0.31^{* * *}$ \\
BRAF-MDQ & $0.16^{* * *}$ & $0.36^{* * *}$ & $0.23^{* * *}$ & $0.24^{* * *}$ & $0.21^{* * *}$ \\
\hline
\end{tabular}

$\mathrm{BP}=$ Bodily pain; $\mathrm{BRAF-MDQ}=$ Bristol RA Fatigue Multidimensional Questionnaire; $E \mathrm{Q}-5 \mathrm{D}=$ five-dimensional EuroQol; HAQ-DI = Health Assessment Questionnaire Disability Index; MCS = mental component summary; PCS = physical component summary; SF-36 = 36-item short-form health survey. ${ }^{*}<0.05 ;{ }^{* *} P<0.01 ;{ }^{* * *} P<0.001$.

Supplementary Table 2. Estimated mean PRO scores over time

\begin{tabular}{|c|c|c|c|c|c|c|}
\hline & \multicolumn{5}{|c|}{ GEE estimated marginal means ( $95 \%$ Wald $\mathrm{CI})$} & \multirow{2}{*}{$\begin{array}{c}\text { Group* } \\
\text { time } \\
P\end{array}$} \\
\hline & Baseline & 3 months & 6 months & 9 months & 12 months & \\
\hline SF-36 PCS & & & & & & $<0.001$ \\
\hline Stop & 45.6 (44.8 to & 43.3 (42.2 to & 43.1 (42.3 to & 43.2 (42.3 to & $43.6(42.7$ & \\
\hline Continue & 46.4) & 44.2) & $44.0)$ & 44.0) & to 44.4$)$ & \\
\hline t-test $P$ & 45.3 (44.2 to & 44.8 (43.8 to & 44.7 (43.6 to & 44.6 (43.4 to & 44.8 (43.7 to & \\
\hline \multirow[t]{4}{*}{ ES $(95 \% \mathrm{Cl})$} & 46.3) & 45.9) & 45.8) & 45.7) & 45.9) & \\
\hline & 0.621 & 0.030 & 0.015 & 0.124 & 0.101 & \\
\hline & 0.03 (-0.11 to & $-0.15(-0.30$ to & $-0.16(-0.30$ to & $-0.14(-0.28$ to & $-0.12(-0.27$ & \\
\hline & $0.18)$ & $-0.01)$ & $-0.01)$ & $0.00)$ & to 0.02 ) & \\
\hline SF-36 MCS & & & & & & 0.003 \\
\hline Stop & 52.0 (51.1 to & 50.6 (49.6 to & 50.6 (49.7 to & 50.6 (49.6 to & 51.3 (50.4 to & \\
\hline Continue & $52.8)$ & 51.6) & 51.6) & 51.5) & $52.2)$ & \\
\hline t-test $P$ & 51.7 (50.4 to & 52.5 (51.4 to & 51.9 (50.7 to & 51.8 (50.5 to & 51.3 (49.9 to & \\
\hline \multirow[t]{4}{*}{ ES (95\% Cl) } & 53.0) & 53.7) & 53.0) & 53.1) & 52.6) & \\
\hline & 0.631 & 0.009 & 0.325 & 0.189 & 0.577 & \\
\hline & $0.03(-0.12$ to & $-0.17(-0.32$ to & $-0.11(-0.25$ to & $-0.12(-0.26$ to & $0.00(-0.14$ & \\
\hline & $0.17)$ & $-0.03)$ & $0.03)$ & $0.03)$ & to 0.15$)$ & \\
\hline
\end{tabular}




\section{GEE estimated marginal means (95\% Wald CI)}

\begin{tabular}{|c|c|c|c|c|c|c|}
\hline & Baseline & 3 months & 6 months & 9 months & 12 months & $P$ \\
\hline SF-36 BP & & & & & & $<0.001$ \\
\hline Stop & 73.9 (72.1 to & 63.7 (61.6 to & $63.9(62.0$ to & $64.3(62.2$ to & $66.9(64.9$ & \\
\hline Continue & 75.6) & 65.7) & 65.9) & 65.9) & to 68.9$)$ & \\
\hline t-test $P$ & 72.7 (70.2 to & 70.6 (68.2 to & 69.6 (67.1 to & 68.5 (65.9 to & 68.8166 .3 & \\
\hline \multirow[t]{4}{*}{ ES $(95 \% \mathrm{Cl})$} & 75.2) & 73.0) & 72.0) & 71.2) & to 71.4 ) & \\
\hline & 0.505 & $<0.001$ & $<0.001$ & 0.020 & 0.221 & \\
\hline & $0.06(-0.09$ to & $-0.30(-0.44$ to & $-0.25(-0.40$ to & $-0.18(-0.33$ to & $-0.09(-0.23$ & \\
\hline & $0.20)$ & $-0.16)$ & $-0.11)$ & $-0.04)$ & to 0.06$)$ & \\
\hline$E Q-5 D$ & & & & & & 0.125 \\
\hline Stop & 0.83 (0.82 to & 0.79 (0.77 to & 0.79 (0.78 to & 0.81 (0.79 to & $0.81(0.80$ & \\
\hline Continue & $0.85)$ & $0.80)$ & $0.81)$ & $0.82)$ & to 0.83 ) & \\
\hline t-test $P$ & 0.85 (0.83 to & $0.84(0.82$ to & 0.82 (0.80 to & 0.83 (0.81 to & 0.83 (0.81 to & \\
\hline \multirow[t]{4}{*}{ ES $(95 \%$ Cl) } & $0.87)$ & $0.86)$ & 0.84) & 0.85) & 0.85) & \\
\hline & 0.287 & $<0.001$ & 0.064 & 0.158 & 0.432 & \\
\hline & $-0.08(-0.23$ to & $-0.24(-0.39$ to & $-0.14(-0.28$ to & $-0.12(-0.27$ to & $-0.09(-0.24$ & \\
\hline & $0.06)$ & $-0.10)$ & 0.01) & $0.02)$ & to 0.11$)$ & \\
\hline HAQ-DI & & & & & & 0.013 \\
\hline Stop & 0.61 (0.55 to & 0.68 (0.62 to & $0.70(0.64-0.75)$ & $0.68(0.63-$ & 0.69 (0.63 - & \\
\hline Continue & $0.66)$ & $0.73)$ & $0.58(0.51-$ & $0.74)$ & $0.74)$ & \\
\hline t-test $P$ & 0.59 (0.52 to & 0.59 (0.51 to & $0.66)$ & 0.6010 .52 - & 0.59 (0.51 - & \\
\hline \multirow[t]{4}{*}{ ES $(95 \%$ Cl) } & 0.66) & 0.66) & 0.013 & $0.67)$ & 0.66) & \\
\hline & 0.759 & 0.024 & 0.18 (0.04 to & 0.063 & 0.047 & \\
\hline & $0.03(-0.11$ to & 0.14 (0.00 to & $0.33)$ & $0.13(-0.01$ to & 0.15 (0.01 to & \\
\hline & $0.17)$ & $0.28)$ & & $0.28)$ & $0.30)$ & \\
\hline BRAF-MDQ & & & & & & 0.008 \\
\hline Stop & 14.9 (13.8 to & 17.2 (15.9 to 18.4$)$ & 17.0 (15.8 to 18.1) & 16.9 (15.7 to & 16.5 (15.3 to & \\
\hline Continue & 15.9) & 15.2 (13.8 to 16.7) & 15.8 (14.3 to & 18.0) & 17.7) & \\
\hline t-test $P$ & 15.5 (14.0 to & 0.022 & 17.3) & $16.2(14.7$ to & 15.8 (14.3 to & \\
\hline \multirow[t]{4}{*}{ ES $(95 \% \mathrm{Cl})$} & 17.0) & 0.14 (0.00 to & 0.196 & 17.6) & 17.3) & \\
\hline & 0.489 & $0.28)$ & $0.08(-0.06$ to & 0.996 & 0.816 & \\
\hline & $-0.05(-0.19$ to & & $0.23)$ & $0.05(-0.09$ & $0.05(-0.16$ & \\
\hline & $0.09)$ & & & to 0.20 ) & to 0.20$)$ & \\
\hline
\end{tabular}

$\mathrm{BP}=$ Bodily pain; BRAF-MDQ = Bristol RA Fatigue Multidimensional Questionnaire; EQ-5D = five-dimensional EuroQol; $E S=$ effect size (Cohen's d); GEE = generalized estimating equation; HAQ-DI = Health Assessment Questionnaire Disability Index; MCS = mental component summary; PCS = physical component summary; SF-36 = 36-item shortform health survey. 
Supplementary Table 3. Cumulative percentage of patients experiencing a worsening $\geq \mathbf{M C I D}$ as compared with baseline

\begin{tabular}{|c|c|c|c|c|c|}
\hline & \multicolumn{4}{|c|}{ GEE estimated percentage ( $95 \%$ Wald $\mathrm{CI}$ ) } & \multirow{2}{*}{$\begin{array}{c}\text { Group* } \\
\text { time } \\
P\end{array}$} \\
\hline & 3 months & 6 months & 9 months & 12 months & \\
\hline SF-36 PCS & & & & & 0.187 \\
\hline Stop & $27.6(23.0$ to & 43.9 (38.8 to & 52.2 (47.1 to 57.3) & $55.4(50.3$ to & \\
\hline Continue & 32.8) & 49.2) & 31.1 (24.9 to 38.0$)$ & $60.4)$ & \\
\hline$X^{2}$ test $P$ & 15.9 (11.2 to 22.0$)$ & 25.6 (19.8 to & $<0.001$ & 38.3 (31.6 to 45.5$)$ & \\
\hline \multirow[t]{3}{*}{$\mathrm{RR}(95 \% \mathrm{Cl})$} & $<0.001$ & 32.3) & 1.69 (1.34 to 2.14$)$ & $<0.001$ & \\
\hline & $1.97(1.33$ to & $<0.001$ & & 1.45 (1.19 to 1.77$)$ & \\
\hline & 2.90) & 1.79 (1.36 to 2.36$)$ & & & \\
\hline SF-36 MCS & & & & & 0.030 \\
\hline Stop & $25.4(21.0$ to & 38.3 (33.4 to & 46.0 (40.9 to 51.1) & 49.8 (44.7 to 55.0$)$ & \\
\hline Continue & 30.3) & 43.4) & 36.5 (29.9 to & 44.3 (37.3 to 51.5) & \\
\hline $\mathrm{X}^{2}$ test $P$ & 11.4 (7.4 to 17.2 ) & 26.3 (20.4 to & 43.6) & 0.113 & \\
\hline \multirow[t]{3}{*}{ RR $(95 \% \mathrm{Cl})$} & $<0.001$ & 33.3) & 0.019 & 1.16 (0.96 to 1.40$)$ & \\
\hline & 2.19 (1.40 to 3.44$)$ & 0.014 & 1.28 (1.03 to 1.59) & & \\
\hline & & 1.39 (1.06 to 1.82$)$ & & & \\
\hline SF-36 BP & & & & & 0.282 \\
\hline Stop & 49.5 (44.1 to & 69.3 (64.4 to & 75.8 (71.2 to & 80.0 (75.7 to 83.7) & \\
\hline Continue & 54.9) & 73.8) & 79.8) & 64.0 (57.0 to 70.5$)$ & \\
\hline$X^{2}$ test $P$ & 31.3 (24.9 to & 45.4 (38.3 to & 55.1 (48.0 to & $<0.001$ & \\
\hline \multirow[t]{3}{*}{$\operatorname{RR}(95 \% \mathrm{Cl})$} & 38.5) & 52.7) & 62.0) & 1.25 (1.12 to 1.40$)$ & \\
\hline & $<0.001$ & $<0.001$ & $<0.001$ & & \\
\hline & 1.65 (1.29 to 2.11) & 1.47 (1.25 to 1.74$)$ & 1.37 (1.19 to 1.57$)$ & & \\
\hline$E Q-5 D$ & & & & & 0.552 \\
\hline Stop & 35.8 (30.8 to 41.1) & 50.0 (44.8 to & 57.3 (52.1 to & 62.3 (57.2 to 67.1) & \\
\hline Continue & 22.1 (16.3 to & 55.1) & $62.2)$ & 51.8 (44.5 to 58.9$)$ & \\
\hline $\mathrm{X}^{2}$ test $P$ & 29.2) & 36.9 (30.0 to & 43.8 (36.7 to & 0.013 & \\
\hline \multirow[t]{3}{*}{ RR (95\% Cl) } & 0.001 & $44.2)$ & 51.0) & 1.20 (1.03 to 1.41$)$ & \\
\hline & 1.63 (1.19 to 2.23 ) & 0.010 & $<0.001$ & & \\
\hline & & 1.30 (1.05 to 1.62$)$ & 1.35 (1.12 to 1.62$)$ & & \\
\hline HAQ-DI & & & & & 0.165 \\
\hline Stop & 28.6 (24.1 to & 43.7 (38.8 to & 49.1 (44.1 to 54.1) & 54.9 (49.9 to & \\
\hline Continue & 33.6) & 48.8) & 32.2 (26.0 to & $59.8)$ & \\
\hline $\mathrm{X}^{2}$ test $P$ & 18.7 (13.7 to 25.0) & 25.2 (19.5 to 31.9) & 39.1) & 36.3 (29.8 to 43.4 ) & \\
\hline \multirow[t]{2}{*}{$\operatorname{RR}(95 \% \mathrm{Cl})$} & 0.007 & $<0.001$ & $<0.001$ & $<0.001$ & \\
\hline & 1.58 (1.12 to 2.23$)$ & 1.75 (1.33 to 2.29$)$ & 1.55 (1.23 to 1.94$)$ & 1.48 (1.21 to 1.82$)$ & \\
\hline
\end{tabular}


Supplementary Table 3 CONTINUED. Cumulative percentage of patients experiencing a worsening $\geq \mathrm{MCID}$ as compared with baseline

\begin{tabular}{|c|c|c|c|c|c|}
\hline & \multicolumn{4}{|c|}{ GEE estimated percentage ( $95 \%$ Wald $\mathrm{CI}$ ) } & \multirow{2}{*}{$\begin{array}{c}\text { Group } \\
\text { time } \\
P \\
\end{array}$} \\
\hline & 3 months & 6 months & 9 months & 12 months & \\
\hline BRAF-MDQ & & & & & 0.708 \\
\hline Stop & 22.2 (17.8 to & 37.5 (32.4 to & 46.2 (41.0 to & 51.1 (45.8 to 56.4 ) & \\
\hline Continue & 27.3) & 42.8) & 51.5) & 33.5 (26.8 to & \\
\hline$X^{2}$ test $P$ & $13.0(8.6$ to 19.0$)$ & 20.8 (15.3 to 27.7$)$ & $27.4(21.2$ to & 40.9) & \\
\hline \multirow[t]{3}{*}{$\operatorname{RR}(95 \% \mathrm{Cl})$} & 0.003 & $<0.001$ & 34.6) & $<0.001$ & \\
\hline & $2.00(1.24$ to & 1.86 (1.33 to 2.60$)$ & $<0.001$ & 1.58 (1.25 to 1.99$)$ & \\
\hline & $3.22)$ & & 1.70 (1.30 to 2.22$)$ & & \\
\hline
\end{tabular}

$\mathrm{BP}=$ Bodily pain; BRAF-MDQ = Bristol RA Fatigue Multidimensional Questionnaire; $E Q-5 D=$ five-dimensional EuroQol; GEE = generalized estimating equation; HAQ-DI = Health Assessment Questionnaire Disability Index; MCS = mental component summary; PCS = physical component summary; RR = relative risk;SF-36 = 36-item short-form health survey.

Supplementary Table 4. Patient-reported experience of stopping with TNFi in patients randomized to the stop group.

\begin{tabular}{lcccc}
\hline & \multicolumn{4}{c}{ Follow-up visit } \\
\cline { 2 - 5 } & $\mathbf{3}$ months & $\mathbf{6}$ months & $\mathbf{9}$ months & $\mathbf{1 2}$ months \\
\hline Very positive & $14.5 \%$ & $13.0 \%$ & $12.1 \%$ & $14.9 \%$ \\
Positive & $30.6 \%$ & $31.5 \%$ & $27.0 \%$ & $23.9 \%$ \\
Neither positive nor negative & $22.6 \%$ & $18.5 \%$ & $20.2 \%$ & $17.6 \%$ \\
Negative & $23.1 \%$ & $20.9 \%$ & $25.0 \%$ & $27.2 \%$ \\
Very negative & $9.1 \%$ & $12.3 \%$ & $15.7 \%$ & $16.4 \%$ \\
\hline
\end{tabular}

Supplementary Table 5. Patient-reported experience of stopping with TNFi in patients randomized to the stop group stratified into restarted or not restarted TNFi.

\begin{tabular}{|c|c|c|c|c|c|c|c|c|}
\hline & \multicolumn{2}{|c|}{$\begin{array}{c}\text { Restarted at } \\
3 \text { months }\end{array}$} & \multicolumn{2}{|c|}{$\begin{array}{c}\text { Restarted at } \\
6 \text { months }\end{array}$} & \multicolumn{2}{|c|}{$\begin{array}{c}\text { Restarted at } \\
9 \text { months }\end{array}$} & \multicolumn{2}{|c|}{$\begin{array}{c}\text { Restarted at } \\
12 \text { months }\end{array}$} \\
\hline & No & Yes & No & Yes & No & Yes & No & Yes \\
\hline Very positive & $19.8 \%$ & $1.8 \%$ & $18.6 \%$ & $4.1 \%$ & $17.1 \%$ & $4.3 \%$ & $25.3 \%$ & $3.7 \%$ \\
\hline Positive & $38.8 \%$ & $10.7 \%$ & $48.1 \%$ & $5.4 \%$ & $41.0 \%$ & $5.0 \%$ & $39.7 \%$ & $6.8 \%$ \\
\hline Neither positive nor negative & $25.6 \%$ & $15.2 \%$ & $21.2 \%$ & $14.3 \%$ & $23.0 \%$ & $15.8 \%$ & $21.3 \%$ & $13.7 \%$ \\
\hline Negative & $14.7 \%$ & $43.8 \%$ & $8.2 \%$ & $40.8 \%$ & $12.9 \%$ & $43.9 \%$ & $10.9 \%$ & $44.7 \%$ \\
\hline Very negative & $1.1 \%$ & $28.6 \%$ & $3.9 \%$ & $35.4 \%$ & $6.0 \%$ & $30.9 \%$ & $2.9 \%$ & $31.1 \%$ \\
\hline
\end{tabular}





\section{Chapter}

\section{An Economic Evaluation of Stopping versus Continuing TNF-Inhibitor Treatment in Rheumatoid Arthritis Patients in Remission or Low Disease Activity: results from the POET randomized trial.}

An Tran-Duy, PhD;

Marjan Ghiti Moghadam, MD;

Martijn AH Oude Voshaar, PhD;

Harald E Vonkeman, MD, PhD;

Annelies Boonen, MD, PhD;

Philip Clarke, PhD;

Geoff McColl, MD, PhD;

Peter M. Ten Klooster, PhD;

TR. Zijlstra, MD, PhD;

Willem F. Lems, MD, PhD;

N Riyazi, MD, PhD;
E.N. Griep, MD, PhD;

JMW Hazes, MD, PhD;

Robert Landewé, MD, PhD;

Hein J. Bernelot Moens, MD, PhD;

Piet L.C.M. van Riel, MD, PhD;

Mart A.F.J. van de Laar, MD, PhD;

TL. Jansen, MD, PhD;

on behalf of the Dutch National POET

collaboration. 


\section{ABSTRACT}

\section{OBJECTIVE}

To evaluate, from a societal perspective, the incremental cost-effectiveness of withdrawing tumor necrosis factor inhibitors (TNFis) compared to continuation of these drugs within a one-year randomized trial among patients with rheumatoid arthritis (RA) having longstanding stable disease activity or remission.

\section{METHODS}

Data were collected from a pragmatic, open label trial. Cost-utility analysis was performed using the non-parametric bootstrapping method and a cost-effectiveness acceptability curve was constructed using the net-monetary benefit (NMB) framework, where a willingness-to-accept threshold (WTA) was defined as the minimal cost saved that a patient accepted for each quality-adjusted life year (QALY) lost.

\section{RESULTS}

531 patients were randomized to the Stop Group and 186 patients to the Continue Group. Withdrawal of TNFis resulted in more than $60 \%$ reduction of the total drug cost, but led to an increase of about 30\% in the other healthcare expenditure. Compared to continuation, stopping TNFis resulted in a mean yearly cost saving of $€ 7,133(95 \% \mathrm{Cl}$, [€6,071, €8,234]) and was associated with a mean loss of QALYS of 0.02 (95\% Cl, [0.002, 0.040]). Mean saved cost [95\% Cl] per QALY lost and per extra flare incurred in the Stop group compared to the Continuation group was $€ 368,269[€ 155,132, € 1,675,909]$ and $€ 17,670[€ 13,650$, $€ 22,721]$, respectively. At a WTA of $€ 98,438$ per QALY lost, the probability that stopping TNFis is cost-effective was $100 \%$.

\section{CONCLUSION}

Although an official WTA is not defined, the mean saved cost of $€ 368,269$ per QALY lost seems acceptable in The Netherlands, given existing data on the willingness-to-pay.

\section{KEYWORDS}

Rheumatoid arthritis; Anti TNF; Health Economic Evaluations. 
Rheumatoid arthritis (RA) is a progressive, immune-mediated inflammatory disease that has a prevalence of around $1 \%$ in developed countries (1). The disease is characterized by synovial inflammation and with time may involve articular damage, disability and extraarticular manifestations. Besides its negative impact on the health of individual patients, RA imposes a significant and increasing economic burden on health-care systems and societies in the form of healthcare resource utilization and (paid) productivity loss ${ }^{(2)}$.

The main goal in the treatment of RA is to suppress inflammatory activity to control pain and prevent unfavorable outcomes such as structural damage and functional disability. Accumulating evidence suggests that optimal clinical outcomes may be achieved if treatment is started early and adjusted to reach predefined disease activity targets $(3,4)$. The subpopulation of patients receiving biological disease modifying anti-rheumatic drugs (bDMARDs) including tumor necrosis factor inhibitors (TNFis) in this treat-to-target strategy has increased over time and accounted for up to $20 \%$ of the population of RA patients in various Western healthcare systems ${ }^{(5,6)}$. Although it is widely believed that the introduction of bDMARDs has contributed to the overall improved clinical picture of severe RA, particularly in the methotrexate refractory population ${ }^{(7)}$, their high cost has raised the question of whether bDMARDs could be discontinued in patients who achieved long-term stable controlled disease, without negatively affecting their health ${ }^{(8)}$. As healthcare budgets are limited and money can be spent only once, savings from stopping treatment with bDMARDs could be used to reinvest in other treatments or increase access to bDMARDs to a larger proportion of the population of patients with RA. The recently completed Potential Optimalisation of Expediency and Effectiveness of TNFis (POET) trial aimed to evaluate the clinical course of patients withdrawn from their TNFis, compared with that of patients who remained on TNFis ${ }^{(9)}$. The results showed that, patients who were withdrawn from their TNFis were more than three times as likely to experience a disease flare compared with patients who continued their TNFis. However, disease control could typically be quickly regained upon TNFi restart. Although the withdrawal of TNFis is evidently associated with lower medication costs, this may be offset by higher non-drug related healthcare costs or by lasting impact on patients' overall quality of life. From the health economic point of view, it is currently unclear whether the benefits of discontinuation of TNFis outweighs the harms. The present study aimed to evaluate, from a societal perspective, the one-year trial based cost-utility and cost-effectiveness of withdrawing TNFis compared to continuation of these drugs in RA patients with longstanding stable disease to inform rheumatologists and patients about balance between savings and health forgone. 


\section{PATIENTS AND METHODS}

\section{STUDY DESIGN AND PATIENTS}

The study outcomes and design of the POET study (NTR3112) are described in detail elsewhere ${ }^{(9)}$. Briefly, this pragmatic, open label trial was performed at 47 rheumatology centers in The Netherlands and included 817 adult patients fulfilling the American College of Rheumatology 1987 classification criteria for RA who were treated with TNFis for at least one year. In addition, patients met one of the following criteria: (1) DAS28 < 3.2 for at least 6 months preceding inclusion ( $n=627$ ), or (2) perceived by the rheumatologists as having remission or low disease activity for at least 6 months prior to inclusion, with DAS28<3.2 at baseline and C-reactive protein level $<10 \mathrm{mg} / \mathrm{L}$ at least once in the six-month period prior to the inclusion $(n=145)$. Patients were randomized to either Stop group $(n=551)$ or Continuation group ( $n=286$ ). After inclusion, TNFis were withdrawn in the Stop group but maintained in the Continuation group. Any other treatment decisions were made by rheumatologists with their patient and continued unchanged as much as possible in both groups. The primary outcome of the study was occurrence of disease flares, defined as DAS28 increase $\geq 0.6$ compared to the baseline and the current DAS28 level $\geq 3.2$.

\section{FOLLOW-UP PROCEDURES}

Patients were assessed at baseline and at least once every 3 months thereafter, for a period of 1 year. At each visit, components of DAS28, Health Assessment Questionnaire Disability Index (HAQ-DI) (10, 11) and EuroQol 5-dimension 3-level (EQ-5D-3L) questionnaire (12) were evaluated, laboratory tests conducted, and patient-reported outcomes collected. Patient reported outcomes included adverse events, days of sick leave and frequencies of healthcare resource utilization. Restart of a TNFi was allowed when a flare occurred; for ethical reasons this included cases where patient's perception of a flare could not be objectively verified.

\section{HEALTH ECONOMIC OUTCOMES}

At each visit, patients answered the EQ-5D-3L questionnaire and reported the frequencies of healthcare resource utilization and number of days of sick leave (in those with a paid job) during the past 3 months. The healthcare resource utilization included visits to rheumatologists and general practitioners, visits to nurse specialists, physiotherapists and psychologists, numbers of diagnostic and laboratory tests, days in hospital and hours of formal and informal care.

For each patient, their health utility at 3-month visits was computed using the Dutch tariffs for EQ-5D-3L ${ }^{(13)}$, and quality-adjusted life years (QALYS) computed as the area under the EQ- 
5D-3L curve. Non-drug direct costs were calculated based on patient reported frequencies of healthcare resource utilization. The unit costs were retrieved from the Dutch Guideline for Economic Evaluations in Healthcare. Drug costs were calculated based on the doses of drug used and the medication prices. Indirect costs of those patients with a paid job were calculated using the friction-cost method with a 3 month-friction period and based on the number of hours absent from work and the average wage per hour for each age group and gender ${ }^{(14)}$. The unit costs and prices published before the current year (2016) were adjusted to the current year using the consumer price index for the Netherlands ${ }^{(15)}$. Costs were not discounted because of the short time horizon of 12 months.

\section{STATISTICAL AND COST-EFFECTIVENESS ANALYSES}

Between 10\% and 15\% of observations contained missing values for costs or utilities (see Supplementary document for details). For the cost-effectiveness analysis, these were replaced with estimates using multiple imputation (MI) as recommended by the International Society for Pharmacoeconomics and Outcomes Research (ISPOR) for costeffectiveness analysis alongside clinical trials ${ }^{(16)}$. For each of the 10 imputed data sets, cost-effectiveness analysis (CEA) was performed using the non-parametric bootstrapping method ${ }^{(17)}$. Five thousand bootstrap samples were generated, from which the expected values of costs and QALYS over the one-year follow-up in each treatment group and of the ratios of incremental cost to incremental QALYs or flares (ICERS) were computed ${ }^{(18)}$. Manca et al recommended an adjustment of QALYs before calculation of ICERs when there was an imbalance in the mean baseline health utility between two trial arms ${ }^{(19)}$. Because mean baseline health utility and other patient characteristics in our study were almost equal between the Stop and Continuation groups, in the main analyses and presentation of the results we used QALYS and costs unadjusted for these negligible differences. To examine the effect of QALYS and costs adjustment on ICERs, we used the regression-based method proposed by Manca et al ${ }^{(19)}$, in which linear regression models for patient-specific QALYS and costs were fitted to the observed data with predictors for QALYs being treatment and baseline health utility, and predictors for costs being treatment, age, sex, disease duration, DAS28 and HAQ-DI. Then, we used the coefficients for the treatment as the differential QALYS and costs for the adjustment. We applied a Box-Cox transformation for QALYS and a log-transformation for costs to meet the assumptions of normal distribution and equal variance of the error term in the linear models ${ }^{(20)}$. The results were pooled across imputed datasets using Rubin's rules (21) to take into account the uncertainty introduced by the missing data. Because the distribution of costs was skewed, the "approximate bootstrap confidence" (ABC) algorithm ${ }^{(22)}$ was used to estimate the confidence intervals (Cls), instead of the usual symmetric confidence interval proposed by Rubin (21). For each imputed data set, a confidence density curve was constructed for each of the outcome variables. The 
10 confidence density curves were then combined by averaging the $y$-values to obtain the average density function, based on which the $95 \% \mathrm{Cl}$ was established by determining the areas under the curve that correspond to 5\% and 95\% percentiles. For each imputed data set, a cost-effectiveness acceptability curve (CEAC) was constructed using the netmonetary benefit (NMB) framework ${ }^{(23)}$, where an expected NMB was calculated as the difference between the willingness-to-accept threshold (WTA) for each QALY lost times the mean QALYS $\left(\bar{E}_{i} \mid \bar{E}_{i}\right)$, and the mean cost obtained from each bootstrap replication $\left(\overline{C_{i}} \mid \bar{C}_{i}\right.$ ) : NMB $=W T A \times \bar{E}_{i}-\bar{C}_{i} N M B=W T A \times \bar{E}_{i}-\bar{C}_{i}$. The Consolidated Health Economic Evaluation Reporting Standards (CHEERS) were followed to report the present study (see the checklist in the supplemental document).

\section{RESULTS}

Baseline characteristics were similar in both groups (Table 1). The majority of the patients had longstanding, erosive disease and utility scores reasonably close to general population norms (24). The sample was further characterized by low disease activity at baseline according to DAS28, as per the inclusion criteria and low disability according to HAQ-DI.

\section{HEALTH OUTCOMES}

Mean DAS28 and HAQ-DI scores in the Continuation Group were almost stable over time (Figure 1), while in the Stop group mean DAS28 score increased from baseline to month 3 and then gradually decreased during the rest of the year, and mean HAQ-DI score slightly increased over time. Post-hoc analyses revealed significant differences in DAS28 scores at all follow up visits ( $p$-values $<0.01$ ), except baseline ( $p$-value $=0.27$ ). No significant difference in HAQ-DI scores between the two groups at any time point was observed.

The percentages of patients with 1 or 2 flares within 12 months was $41.1 \%$ and $8.1 \%$ in the Stop Group and $15.4 \%$ and $1.4 \%$ in the Continuation group. No patients and only $0.6 \%$ of patients had 3 flares in the Continuation and Stop groups, respectively. On average, within 12 months each patient experienced 0.59 flares $(95 \% \mathrm{Cl},[0.53,0.64])$ in the Stop group, and $0.18(95 \% \mathrm{Cl},[0.13,0.24])$ flares in the Continuation group.

Mean health utility in the Continuation group slightly deteriorated during the first 6 months and then remained relatively stable during the rest of the year, while that in the Stop group decreased within the first 3 months and then slightly increased until end of the year (Figure 2). Except for month 3 ( $p$-value $=0.0005$ ), mean health utility was not significantly different at any time points between the two groups ( $p$-values $>0.05$ ). 
Table 1. Baseline patient characteristics

\begin{tabular}{lll}
\hline Characteristic & Stop group $(\mathrm{n}=531)$ & Continuation group $(\mathrm{n}=286)$ \\
\hline Mean age (SD) & $60.1(11.8)$ & $59.7(10.6)$ \\
Female, no. (\%) & $362(68)$ & $188(66)$ \\
Mean disease duration in years (SD) & $12.0(8.8)$ & $11.1(8.4)$ \\
RF positive, no. (\%) & $238(67.5)$ & $178(67.4)$ \\
Anti-CCP positive, no. (\%) & $332(68.3)$ & $179(67.8)$ \\
Erosive disease, no. (\%) & $305(62.8)$ & $152(57.6)$ \\
Mean DAS28 (SD) & $1.98(0.76)$ & $2.05(0.73)$ \\
Mean HAQ (SD) & $0.63(0.59)$ & $0.62(0.55)$ \\
Mean EQ-5D-3L score (SD) & $0.83(0.16)$ & $0.84(0.13)$ \\
Patient on a TNF-inhibitor, no (\%) & & \\
Adalimumab & $271(51.1)$ & $129(45.1)$ \\
Etanercept & $213(40.2)$ & $133(46.5)$ \\
Infliximab & $25(4.7)$ & $14(4.9)$ \\
Golilmumab & $15(2.8)$ & $8(2.8)$ \\
Certolizumab & $7(1.2)$ & $2(0.7)$ \\
\hline
\end{tabular}

$\mathrm{RF}=$ rheumatoid factor; Anti-CCP = anti-cyclic citrullinated peptide antibodies; DAS28 = disease activity score in 28 joints; $\mathrm{HAQ}=$ Health Assessment Questionnaire; EQ-5D-3L = five-dimensional 3-level EuroQol; $T$ TF = tumor necrosis factor-alpha.
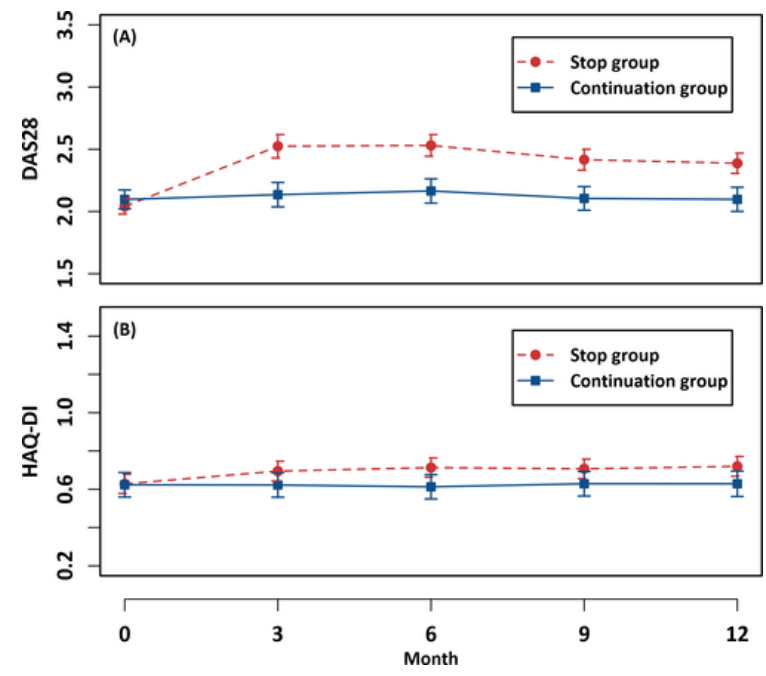

Figure 1. Mean DAS28 and HAQ-DI at different points in time during the 1-year follow-up in the two treatment strategies. The vertical bars represent $95 \%$ confidence intervals. 


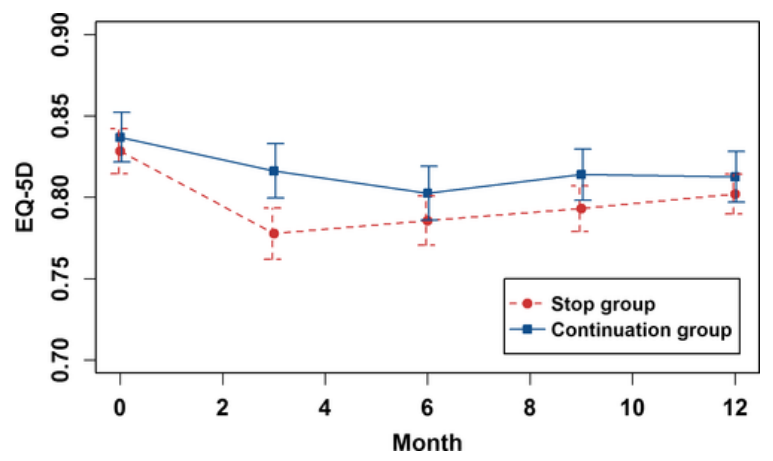

Figure 2. Mean EQ-5D-3L scores at different points in time during the 1-year follow-up in the two treatment strategies. The vertical bars represent $95 \%$ confidence intervals.

\section{HEALTHCARE, MEDICATION AND SICK LEAVE COSTS AND QALYS}

Table 2 shows means and 95\% Cls of non-drug healthcare costs, drug costs and sick leave costs (in Euro) and of QALYs per patient per year in the two treatment strategies. A detailed overview of healthcare resource utilization in each category at baseline and cumulative over 1 year as well as the corresponding unit prices is provided in the supplementary document (Tables S1-S5). Withdrawal of TNFis resulted in more than $60 \%$ reduction of the total drug cost, but led to an increase of about 30\% of the other healthcare expenditure. Sick leave cost in the Stop group was slightly lower than that in the Continuation group, although the difference between the two groups was insignificant. Since the cost of TNFis was much larger than the increased expenditure in other cost components, the mean total cost incurred by each patient per year in the Continuation group (€14,740;95\% Cl, [€13,913, $€ 15,676])$ was almost double that in the Stop group (€7,607; 95\% Cl, [€7000, €8,261]). Mean QALYs [95\% Cls] per patient in the Stop and Continuation groups were 0.79 [0.781, $0.805]$ and 0.81 [0.799, 0.829], respectively. 
Table 2. Mean costs (in Euro) and QALY [95\% Cls] per patient per year in the two treatment strategies

\begin{tabular}{|c|c|c|c|}
\hline & Stop group & Continuation group & Incremental \\
\hline \multirow[t]{2}{*}{ Non-drug health care cost } & 2,122 & 1,663 & 459 \\
\hline & {$[1,684,2,563]$} & {$[1,384,1,958]$} & {$[70,890]$} \\
\hline \multirow[t]{2}{*}{ Drug cost } & 4,894 & 12,450 & $-7,556$ \\
\hline & {$[4,3965,402]$} & {$[11,427,13,472]$} & {$[-8,547,-6,629]$} \\
\hline \multirow[t]{2}{*}{ CSDMARD } & 325 & 324 & 1 \\
\hline & {$[220,461]$} & {$[230,471]$} & {$[-98,121]$} \\
\hline \multirow[t]{2}{*}{ TNFi } & 4,568 & 12,126 & $-7,558$ \\
\hline & {$[4,070,5,067]$} & {$[11,106,13,145]$} & {$[-8,534,-6,643]$} \\
\hline \multirow[t]{2}{*}{ Sick leave cost } & 591 & 626 & -35 \\
\hline & {$[450,735]$} & {$[452,804]$} & {$[-226,155]$} \\
\hline \multirow[t]{2}{*}{ Total cost } & 7,607 & 14,740 & $-7,133$ \\
\hline & {$[7,001,8,261]$} & {$[13,913,15,676]$} & {$[-8,234,-6,071]$} \\
\hline \multirow[t]{2}{*}{ QALYS } & 0.79 & 0.81 & -0.022 \\
\hline & {$[0.781,0.805]$} & {$[0.799,0.829]$} & {$[-0.040,-0.002]$} \\
\hline \multirow[t]{2}{*}{ ICER (€ per QALY) } & & & 368,269 \\
\hline & & & {$[155,132,1,675,909]$} \\
\hline
\end{tabular}

CSDMARD, conventional synthetic disease-modifying antirheumatic drug; TNFi, tumor necrosis factor inhibitor; QALYS, quality-adjusted life years; ICER, incremental cost-effectiveness ratio

\section{INCREMENTAL COST-EFFECTIVENESS AND UNCERTAINTY}

An average patient in the Stop group may save $€ 7,133$ (95\% Cl, [€6,071, €8,234]) for society, but would lose 0.022 QALY (95\% Cl, [0.002, 0.040]) per year and experience 0.41 (95\% Cl, $[0.33,0.48])$ more flares compared to an average patent in the Continuation group. Mean saved cost [95\% Cl] per QALY lost and per extra flare incurred in the Stop group compared to the Continuation group was $€ 368,269[€ 155,132$, $€ 1,675,909]$ and $€ 17,670[€ 13,650$, $€ 22,721]$, respectively. When QALYS and costs were adjusted for differences in baseline health utility and patient characteristics, mean saved cost [95\% Cl] per QALY lost in the Stop group compared to the Continuation group (€371,457 [€156,291, €1,736,887]) was slightly higher than that when no adjustment was made. Because the difference in mean sick leave costs between the two groups was very small, mean costs saved [ $95 \% \mathrm{Cl}$ ], from the healthcare perspective, per QALY lost (€366,642 [€152,396, €1,662,057]) and per flare increase $(€ 17,587[€ 13,575, € 22,642])$ were similar to those from the societal perspective. Hereafter, we focused on the outcomes and interpretation from the societal perspective. The scatter plot of the incremental mean costs and QALYs resulting from 50,000 bootstrapped replications for 10 imputed data sets is provided in Figure 3. All differences in mean costs were negative. About $1.5 \%$ of all data points fell in the south-east quadrant 
(i.e. saved cost with increased QALYS), indicating that the probability that withdrawal of TNF-inhibitors is cost-effective at any level of WTA is negligible. At WTAs of $€ 330,450$ and $€ 98,438$, the probabilities that withdrawal of TNF-inhibitors were cost-effective were 0.5 and 1.0, respectively (Figure 4). If stopping TNFis is considered as the baseline comparator, it was almost certain that the continuation of TNFis was not cost-effective at a willingnessto-pay (WTP) of $€ 100,000$ per QALY gained.

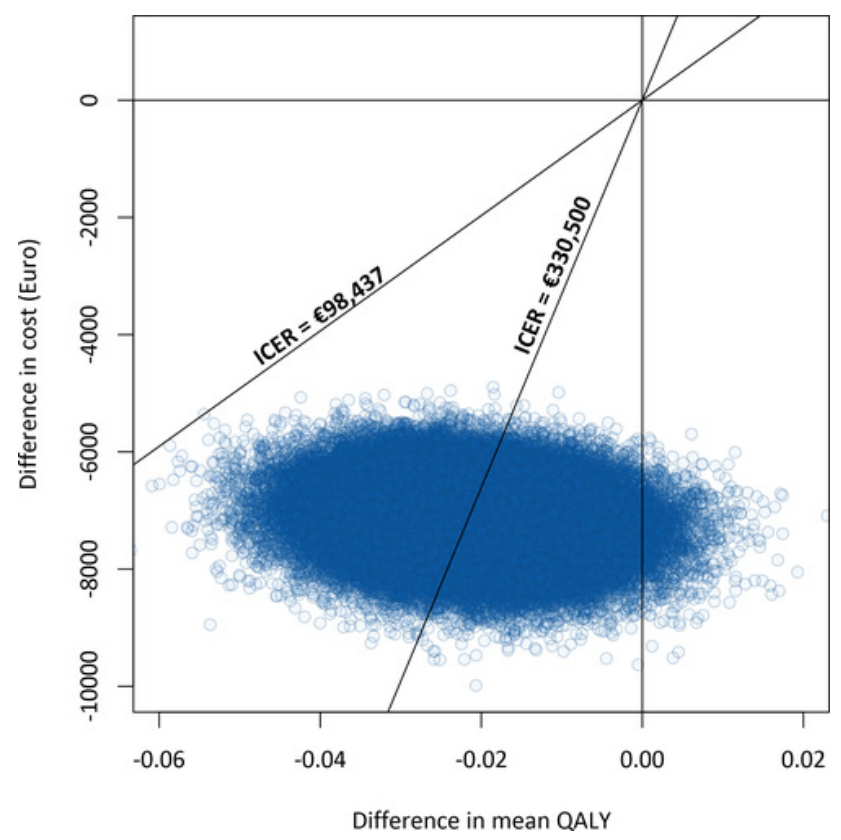

Figure 3. Scatter plot of incremental mean cost against incremental mean QALYs of Stop strategy compared to Continuation strategy. Each data point was obtained from one bootstrap replication.

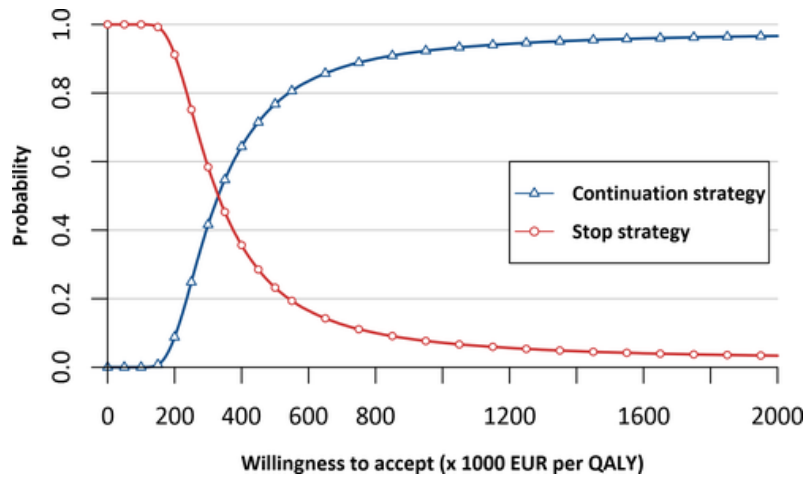

Figure 4. Cost-effectiveness acceptability curve for Continuation and Stop strategies. 


\section{DISCUSSION}

Our analysis shows that stopping TNFis in RA patients with stable controlled disease (remission or low disease activity) can save considerable cost but results in a small QALY loss. At the beginning of this century, TNFis were developed, studied and introduced to control inflammatory disease activity in patients with RA. Despite increasing budget impact over the first fifteen years since the introduction of these drugs, evidence on the possibility to discontinue TNFi in the maintenance phase is still sparse. Therefore, patients are frequently kept on TNFi indefinitely. Up to now, TNFis have been recommended in most of the developed countries for management of RA and reimbursed for patients with persistently high disease activity despite adequate treatment with at least 2 csDMARDs (25-28). These recommendations were predominantly based on evidence on the favorable cost-effectiveness ratio of TNFi therapy compared to csDMARDs in patients with severe disease. For instance, a recent systematic literature review on economic aspects of treatment with TNFis to inform clinical recommendations by the European League Against Rheumatism (EULAR) showed that the incremental cost of the use of a TNFi after failure of 2 csDMARDs per QALY gain was less than $€ 60,000$ (conversion applied) in 14 out of 18 studies ${ }^{(29)}$. Regardless of the majority of studies with outcomes in favor of the use of TNFis in this population, concerns continue to be raised about the substantial impact of therapy with TNFis on the healthcare budgets owing to the high prices of these drugs. For economic reasons, withdrawal of TNFis has therefore been considered in patients with sustained low disease activity.

So far, about a dozen studies examining the effects of discontinuing TNFis on clinical outcomes have been published (30-35). Due to the heterogeneity in study designs, characteristics of patients, definitions of low disease activity and permission of restarting TNFis when failure was observed, the results between studies differed remarkably; the proportions of patients who were free from failure after discontinuation of TNFis ranged from $0 \%$ to $33 \%$ at 7 months, and from $13 \%$ to $80 \%$ at 12 months ${ }^{(31)}$. Despite the fact that high treatment costs are a frequently cited motivation to conduct such studies, unfortunately no previous discontinuation studies reported on preference-based health valuation such as EQ-5D, or healthcare costs which are important to inform health policy decisions. In the present study, we showed that, except for the first follow-up point after baseline, the mean health utility did not significantly differ over time between patients in the Stop and Continuation groups. These results suggest that discontinuing TNFis in patients with long-term stable disease is a strategy that over the course of 1 year is associated with considerable saved cost and negligible loss of quality of life. Very similar conclusions were reached in the DRESS down titration study in which it was found that when using a 
TNFi down titration approach guided by disease activity, an amount of $€ 390,493$ could be saved for each QALY lost compared with continued tight control treatment. Together, these findings suggest significant potential for disinvestment decisions (i.e. to stop subsidizing therapies that are not cost-effective) ${ }^{(36)}$. This could potentially free up resources that could be reallocated to other more cost-effective interventions. One way to do this would be to look for scope for implementing other more cost-effective interventions on the patients formerly receiving TNFis, as any improvement in their health status could offset the loss in QALYs due to discontinuation. Such an approach would avoid having to make trade-offs involving reducing the health of patients with RA.

While there is no explicit WTA threshold that could be used to judge whether or not TNFis should be discontinued it is useful to consider the threshold for WTP which is suggested to be between $€ 20,000$ and $€ 73,000$ per QALY in the Netherlands ${ }^{(37)}$. The estimated saving per QALY lost of $€ 368,269$ is much higher than the maximal bound of this threshold range, suggesting that it would be cost-effective to discontinue TNFis in the maintenance phase while the patients are in a state of low disease activity or remission. Adjustment of QALYS and costs for differences in baseline health utility and patient characteristics resulted in a slightly higher saving than the above-mentioned value, which increases the likelihood that stopping TNFis is cost-effective.

Our study has strengths and weaknesses. It is based on the largest RCT on discontinuing TNFis in RA patients in stable low disease activity, with high-quality data owing to a strictly electronic data collection protocol. Generalizability of the results to the overall population of patients withdrawing from TNFis is probably high, since it was a pragmatic trial with relatively few inclusion and exclusion criteria were maintained in POET study. We used advanced methods, i.e. combination of multiple imputation, bootstrap and ABC algorithm, to capture the uncertainty surrounding the study results. However, our findings on the costeffectiveness of stopping TNFis are valid only for an intervention duration of 12 months. Studies on the WTA thresholds in patients with RA are important to support decision making. Since patients with different disease durations and numbers of failed TNFis may respond differently to the discontinuation of the current TNFi, more research of response to discontinuation is needed.

In conclusion, stopping TNFis in RA patients with stable low disease activity, on average, was associated with a cost saving of $€ 7,133$, a loss of 0.022 QALY and an increase of 0.41 flares per patient per year. Although an official WTA threshold is not available, the mean saved cost of $€ 368,269$ per QALY lost we found would be cost-effective in The Netherlands, given existing data on the WTP and the WTA/WTP ratio. If the WTA threshold 
is $€ 100,000$, the probability that stopping TNFis is cost-effective is approximately 1 .

\section{ACKNOWLEDGMENTS}

The authors thank all patients, rheumatology nurses and rheumatologists of the participating centers, the members of the Steering Committee consisting of Renée Allaart (University Medical Center Leiden), Annelies Boonen (Maastricht University Medical Center), Reinhard Bos (Medical Center Leeuwarden), Liesbeth Brouwer (University Medical Center Groningen), Alfons den Broeder (Sint Maartens clinic), Danielle Gerlag (Amsterdam Medical Center), Mieke Hazes (Erasmus University Medical Center), Willem Lems (VU Medical Center), Dirkjan van Schaardenburg (Reade), Janneke Tekstra (University Medical Center Utrecht), and Harald Vonkeman (Arthritis Center Twente MST \& University of Twente), and Gerardine Willemsen (patients association), Huib Kooiman (Dutch Ministry of Health, Welfare \& Sports (VWS)), and Benien Vingerhoeds (Netherlands Organisation for Health Research and Development (ZonMW)). 


\section{REFERENCES}

1. Gabriel SE, Michaud K. Epidemiological studies in incidence, prevalence, mortality, and comorbidity of the rheumatic diseases. Arthritis research \& therapy. 2009;11(3):229.

2. Boonen A, Severens JL. The burden of illness of rheumatoid arthritis. Clinical rheumatology. 2011;30 Suppl 1:S3-8.

3. Smolen JS, Breedveld FC, Burmester GR, Bykerk V, Dougados $M$, Emery $P$, et al. Treating rheumatoid arthritis to target: 2014 update of the recommendations of an international task force. Ann Rheum Dis. 2016;75(1):3-15.

4. Singh JA, Saag KG, Bridges SL, Jr., Akl EA, Bannuru RR, Sullivan MC, et al. 2015 American College of Rheumatology Guideline for the Treatment of Rheumatoid Arthritis. Arthritis \& rheumatology (Hoboken, NJ). 2016;68(1):1-26.

5. Yazici Y, Shi N, John A. Utilization of biologic agents in rheumatoid arthritis in the United States: analysis of prescribing patterns in 16,752 newly diagnosed patients and patients new to biologic therapy. Bulletin of the NYU hospital for joint diseases. 2008;66(2):77-85.

6. Huscher D, Mittendorf $T$, von Hinuber $U$, Kotter I, Hoese G, Pfafflin A, et al. Evolution of cost structures in rheumatoid arthritis over the past decade. Ann Rheum Dis. 2015;74(4):738-45.

7. Bongartz $T$, Sutton AJ, Sweeting $M J$, Buchan I, Matteson EL, Montori V. AntiTNF antibody therapy in rheumatoid arthritis and the risk of serious infections and malignancies: systematic review and meta-analysis of rare harmful effects in randomized controlled trials. JAMA. 2006;295(19):2275-85.

8. Scott DL. Biologics-based therapy for the treatment of rheumatoid arthritis. Clinical pharmacology and therapeutics. 2012;91(1):30-43.

9. Ghiti Moghadam M, Vonkeman HE, Ten Klooster PM, Tekstra J, van Schaardenburg D, Starmans-Kool M, et al. Stopping Tumor Necrosis Factor Inhibitor Treatment in Patients With Established Rheumatoid Arthritis in Remission or With Stable Low Disease Activity: A Pragmatic Multicenter, Open-Label Randomized Controlled Trial. Arthritis \& rheumatology (Hoboken, NJ). 2016;68(8):1810-7.

10. Ramey DR, Raynauld JP, Fries JF. The health assessment questionnaire 1992. Status and review. Arthritis Rheum. 1992;5(3):119-29.

11. Bruce B, Fries JF. The health assessment questionnaire (HAQ). Clin Exp Rheumatol. 2005;23(5):14.

12. The EuroQol Group. EuroQol-a new facility for the measurement of health-related quality of life. Health Policy. 1990;16:199208.

13. Lamers LM, McDonnell J, Stalmeier PF, Krabbe PF, Busschbach JJ. The Dutch tariff: results and arguments for an effective design for national EQ-5D valuation studies. Health Econ. 2006;15(10):1121-32.

14. StatLine. Gemiddeld inkomen van personen naar kenmerken en naar regio. Centraal Bureau voor de Statistiek (CBS). Available at http://statline.cbs.nl. Accessed 
10 February 2017. 2017.

15. StatLine. Consumentenprijzen. Centraal Bureau voor de Statistiek (CBS). Available at http://statline.cbs.nl. Accessed 9 February 2017. 2017.

16. Ramsey S, Willke R, Briggs A, Brown R, Buxton M, Chawla A, et al. Good research practices for cost-effectiveness analysis alongside clinical trials: the ISPOR RCTCEA Task Force report. Value in health : the journal of the International Society for Pharmacoeconomics and Outcomes Research. 2005;8(5):521-33.

17. Barber JA, Thompson SG. Analysis of cost data in randomized trials: an application of the non-parametric bootstrap. Statistics in medicine. 2000;19(23):3219-36.

18. Briggs $\mathrm{AH}$, Wonderling $\mathrm{DE}$, Mooney $\mathrm{CZ}$. Pulling cost-effectiveness analysis up by its bootstraps: a non-parametric approach to confidence interval estimation. Health economics. 1997:6(4):327-40.

19. Manca A, Hawkins N, Sculpher MJ. Estimating mean QALYs in trial-based costeffectiveness analysis: the importance of controlling for baseline utility. Health Econ. 2005;14(5):487-96.

20. Box GE, Cox DR. An analysis of transformations. J R Stat Soc Series B (Methodological). 1964:211-52.

21. Rubin DB. Multiple imputation for nonresponse in surveys: John Wiley \& Sons; 2004.

22. Efron B. Missing data, imputation, and the bootstrap. Journal of the American Statistical Association. 1994;89(426):463-
75.

23. Hoch JS, Briggs AH, Willan AR. Something old, something new, something borrowed, something blue: a framework for the marriage of health econometrics and costeffectiveness analysis. Health economics. 2002:11(5):415-30.

24. Cabases J, Janssen B, Szende A. Selfreported population health: an international perspective based on EQ-5D: Springer; 2014.

25. Emery P, Van Vollenhoven R, Ostergaard M, Choy E, Combe B, Graninger W, et al. Guidelines for initiation of anti-tumour necrosis factor therapy in rheumatoid arthritis: similarities and differences across Europe. Ann Rheum Dis. 2009;68(4):456-9.

26. Singh JA, Furst DE, Bharat A, Curtis JR, Kavanaugh AF, Kremer JM, et al. 2012 update of the 2008 American College of Rheumatology recommendations for the use of disease-modifying antirheumatic drugs and biologic agents in the treatment of rheumatoid arthritis. Arthritis Care Res (Hoboken). 2012;64(5):625-39.

27. Smolen JS, Landewe R, Breedveld FC, Dougados M, Emery P, Gaujoux-Viala C, et al. EULAR recommendations for the management of rheumatoid arthritis with synthetic and biological disease-modifying antirheumatic drugs. Ann Rheum Dis. 2010;69(6):964-75.

28. Smolen JS, Landewe R, Breedveld FC, Buch M, Burmester G, Dougados M, et al. EULAR recommendations for the management of rheumatoid arthritis with 
synthetic and biological disease-modifying antirheumatic drugs: 2013 update. Ann Rheum Dis. 2014;73(3):492-509.

29. Schoels $M$, Wong J, Scott DL, Zink A, Richards $\mathrm{P}$, Landewe $\mathrm{R}$, et al. Economic aspects of treatment options in rheumatoid arthritis: a systematic literature review informing the EULAR recommendations for the management of rheumatoid arthritis. Ann Rheum Dis. 2010;69(6):995-1003.

30. Navarro-Millan I, Sattui SE, Curtis JR. Systematic review of tumor necrosis factor inhibitor discontinuation studies in rheumatoid arthritis. Clin Ther. 2013;35(11):1850-61 e1.

31. Yoshida K, Sung YK, Kavanaugh A, Bae SC, Weinblatt ME, Kishimoto M, et al. Biologic discontinuation studies: a systematic review of methods. Ann Rheum Dis. 2014;73(3):595-9.

32. Kavanaugh A, Lee SJ, Curtis JR, Greenberg JD, Kremer JM, Soto L, et al. Discontinuation of tumour necrosis factor inhibitors in patients with rheumatoid arthritis in lowdisease activity: persistent benefits. Data from the Corrona registry. Ann Rheum Dis. 2015;74(6):1150-5.

33. Tanaka Y, Hirata S, Kubo S, Fukuyo S, Hanami K, Sawamukai N, et al. Discontinuation of adalimumab after achieving remission in patients with established rheumatoid arthritis: 1-year outcome of the HONOR study. Ann Rheum Dis. 2015;74(2):389-95.

34. van Vollenhoven RF, Ostergaard $M$, Leirisalo-Repo M, Uhlig $\mathrm{T}$, Jansson $\mathrm{M}$, Larsson E, et al. Full dose, reduced dose or discontinuation of etanercept in rheumatoid arthritis. Ann Rheum Dis. 2016;75(1):52-8.

35. Moghadam MG, Vonkeman HE, ten Klooster PM, Tekstra J, van Schaardenburg D, Starmans-Kool M, et al. Stopping Tumor Necrosis Factor Inhibitor Treatment in Patients With Established Rheumatoid Arthritis in Remission or With Stable Low Disease Activity: A Pragmatic Multicenter, Open-Label Randomized Controlled Trial. Arthritis \& Rheumatology. 2016;68(8):1810-7.

36. Karnon J, Carlton J, Czoski-Murray C, Smith K. Informing disinvestment through costeffectiveness modelling. Applied Health Economics and Health Policy. 2009;7(1):1-9.

37. Raad voor de Volksgezondheig en Zorg. Zicht op zinnige en duurzame zorg. Quantes, Rijswijk. Available at http://rvz.net. Accessed 11 February 2017. 2006. 


\section{SUPPLEMENTARY FILES}
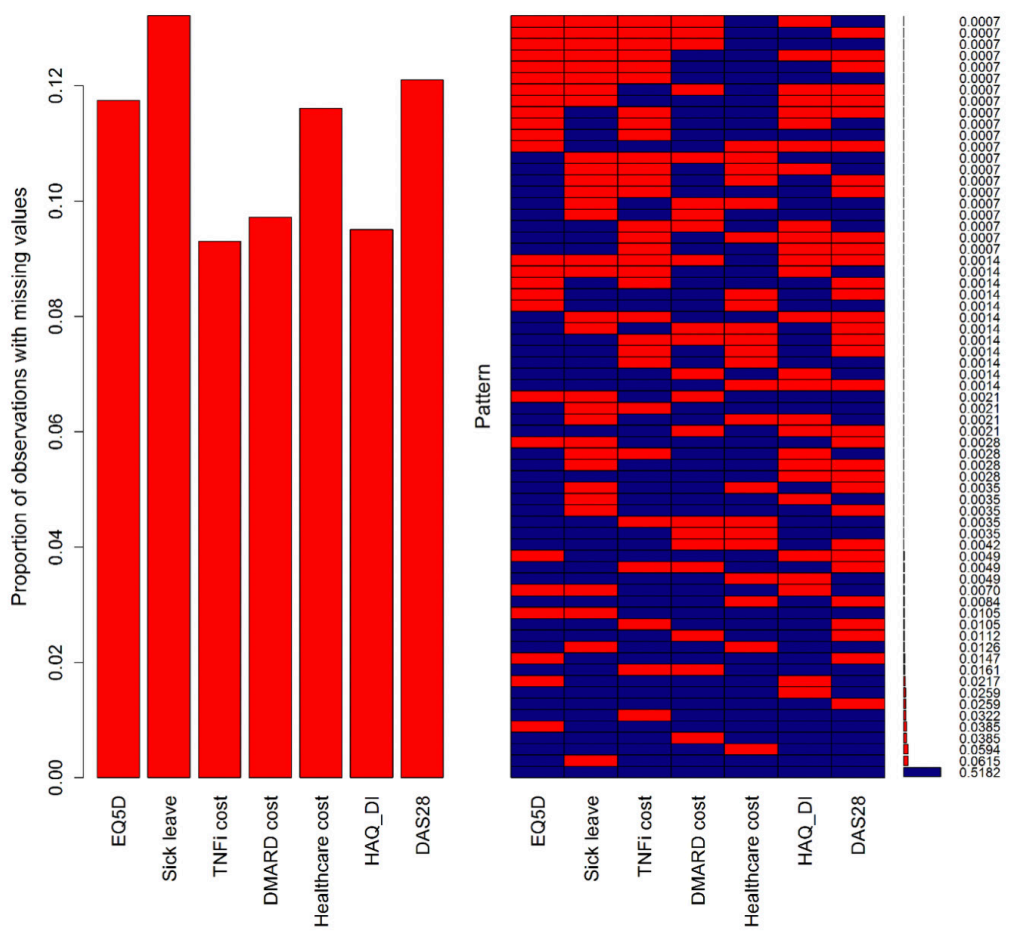

Figure S1. Proportion of all observations containing missing data in each variable and pattern of missing data in the Continuation group 

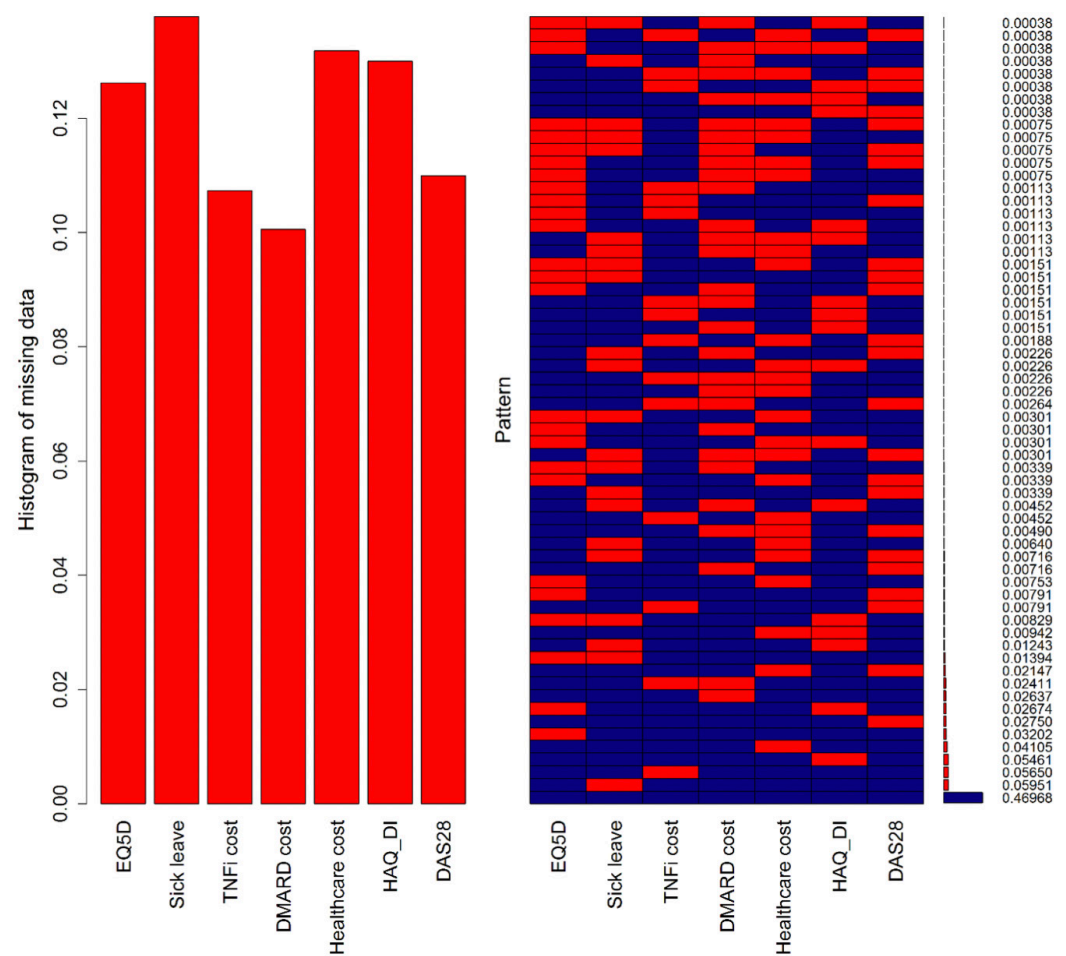

Figure S2. Proportion of all observations containing missing data in each variable and pattern of missing data in the Stop group 

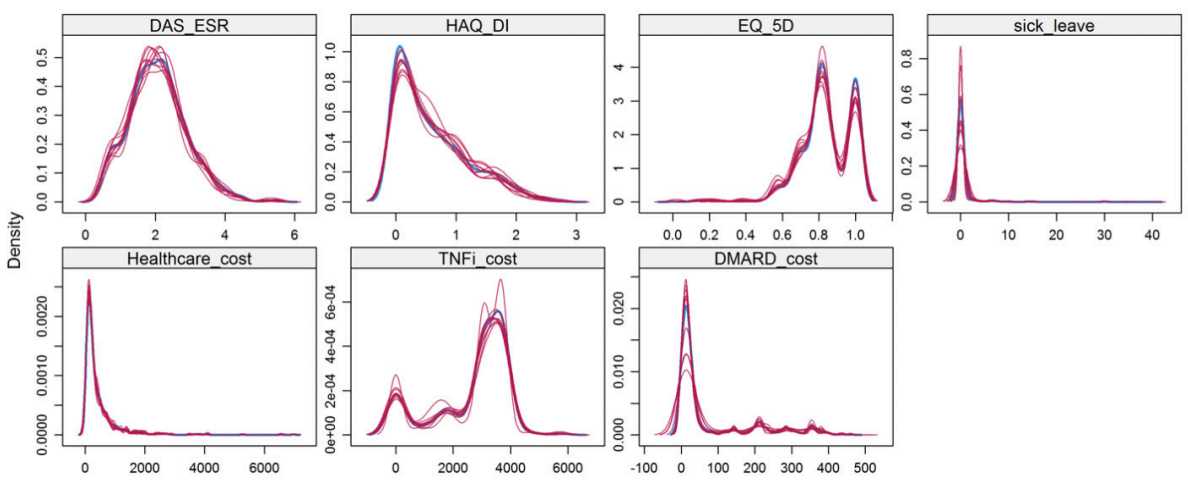

Figure S3. Density of observed data (blue line) versus densities of 10 sets of imputed data (red lines) in each variable in the Continuation group.
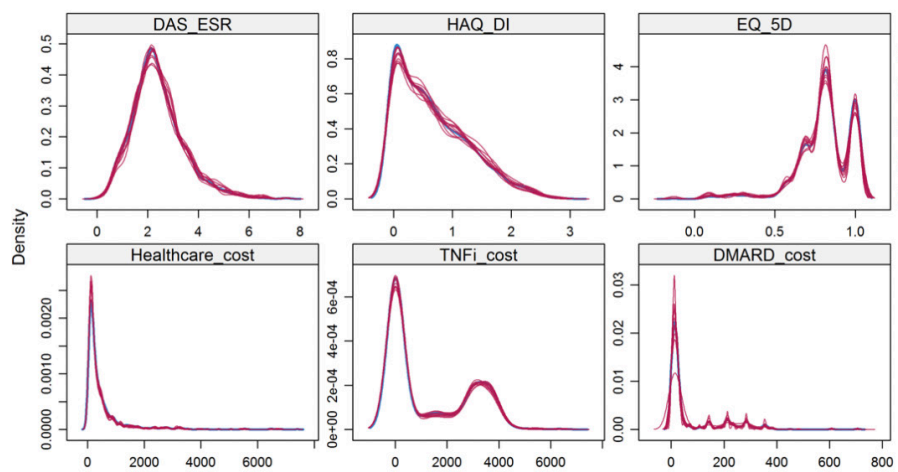

Figure S4. Density of observed data (blue line) versus densities of 10 sets of imputed data (red lines) in each variable in the Stop group 



\section{Chapter}

\section{Measuring Disease Exacerbation and Flares in Rheumatoid Arthritis: Comparison of Commonly used Disease Activity Indices \& Individual Measures.}

Martijn A.H. Oude Voshaar, PhD;

Marjan Ghiti Moghadam, MD,;

Harald E. Vonkeman, MD, PhD;

Peter M. ten Klooster, PhD;

Dirkjan van Schaardenburg, MD, PhD;
Janneke Tekstra, MD, PhD;

H. Visser, MD, PhD;

Mart A.F.J. van de Laar,

Tim L. Jansen, MD, PhD 


\section{ABSTRACT}

\section{Objective}

To evaluate and compare the utilityof commonly used outcome measures for assessing disease exacerbation or flare in patients with rheumatoid arthritis (RA).

\section{Methods}

Data of the Dutch Potential Optimalisation of (Expediency) and Effectiveness of Tumor necrosis factor alpha-blockers (POET) study in which 462 patients discontinued their tumor necrosis factor alpha inhibitor was used. The ability of different measures to discriminate between those with and without physician-reported flare or medication escalation at the three months visit (T2) was evaluated by calculating effect size statistics (ES). Responsiveness to increased disease activity was compared between measures by standardizing changescores (SCS) from baseline to the 3 months visit. Finally the incremental validity of individual outcome measures beyond the SimplifiedDisease Activity Score was evaluated using logistic regression analysis.

\section{Results}

SCS were greater for disease activity indices than for any of the individual measures. The DAS28, CDAl and SDAl performed similarly. Pain and physician and patient global assessment of disease activitywere the most responsive individual measures.Similar results were obtained for discriminative ability, with greatest ES for disease activity indices followed by pain and patient- and physician global. Pain was the only measure to demonstrate incremental validity beyond SDAl in predicting 3 months flare status.

\section{Conclusion}

These results support the use of composite disease activity indices, patient-reported pain and disease activity \& physician-reported disease activity for measuring disease exacerbation or identifying flares of RA. Physical function, acute phase response and the auxiliary measures fatigue, participation and emotional wellbeing performed poorly. 
Disease activity cannotbe directly measured in rheumatoid arthritis (RA), due to the absence of a gold standardmeasure of the inflammatory process of the disease. Consequently, numerous standardized measures of various symptoms and signs of the disease and measures of global disease impact have been proposed and validated over time to assess beneficial effects of treatment. Early clinical trials in this field were characterized by many different outcome measures and results were frequently difficult to compare (1). To address this, a set of seven outcome domains was formally recommended independently by the American College of Rheumatology and the World Health Organization/International League against Rheumatism a few decades ago ${ }^{(2,3)}$. Further efforts to standardize outcomes across studies have led to the subsequent development and validation of several composite disease activity indices. Each of these combines a number of the endorsed outcomes to produce anoveralldisease activity score ${ }^{(4-6)}$. Responsiveness and discriminative ability are key properties of such measures and the performance of commonly used disease activity indices as well as individual core set measures in detecting treatment effects and discriminating between different levels of achieved therapeutic responsehave been extensively described in previous studies ${ }^{(7-14)}$

While these previous studies have provided a great deal of insight into which of the commonly applied instruments are most suitable for measuring treatment benefits in RA, not much is known about their performance when assessing exacerbation of disease activity or flares. This is of importance however, because the occurrence of flares or disease worsening are increasingly adopted asendpoints of clinical trials ${ }^{(15)}$. Moreover there is a widely felt need for instruments that can be used to monitor disease exacerbation in clinical practice ${ }^{(16)}$.

Recently it has been proposed by the OMERACT flare working group thatflare of RA represents any worsening of disease activity that would, if persistent, in most cases lead to initiation or change of therapy; and a flare represents a cluster of symptoms of sufficient duration and intensity to require initiation, change, or increase in therapy ${ }^{(17)}$. Moreover, several consensus based initiatives have endorsed an extended set of outcomes for the assessment of flares intended to comprehensively cover the experience from the patient's perspective ${ }^{(18,19)}$. However, a factor to consider besides the relevance of outcome domains according to experts and patientsis that the assessment of flares or disease worsening should proceed using measurement instruments that are optimally valid and reliable for this purpose, in order to minimize flare status misclassifications (i.e. false positives and false negatives) and non-optimal sensitivity to changedue torandom and systematic measurement errors. 
In the present study we compared the relative efficiency of a series of clinical and patientreported measures using data of the Dutch Potential Optimalisation of (Expediency) and Effectiveness of TNF-blockers (POET)study, in order to identify measurement instruments and outcome domains that most reliably assess disease exacerbation. The primary objective of the present post-hoc study was to compare the performance of commonly used disease activity measures and indices, as well as recently endorsed patient-reported outcome domains in assessing disease activity worsening. A secondary objectiveof the present study was to evaluate the incremental validity of individual outcomes over the traditionaldisease activity indices.

\section{METHODS}

\section{POET STUDY}

POET was an open-label pragmatic randomized controlled trial conducted at 47 rheumatology departments in the Netherlands.To be eligible for the study, patients had to: be 18 years of age or older, meet the American College of Rheumatology1987 revised criteria for the classification of $\mathrm{RA}^{(20)}$, have been treated for at least one year with concomitant tumor necrosis factor alpha inhibitor and conventional synthetic disease modifying anti-rheumatic drugs (DMARD) and be in remission or stable low disease activity for at least six months, defined as either a Disease Activity Score in 28-joints (DAS28) (21) $<3.2$ measured at least twice, or the rheumatologists' clinical impression of remission or stable low disease activity in combination with at least one CRP level $<10 \mathrm{mg} / \mathrm{L}$ in the six months prior to inclusion. There were no exclusion criteria. Study inclusion took place from March 2012 to March 2014. The study was approved by the Institutional Ethical Review Boards of all participating hospitals (Grant/Aware number 40-00506-98-12001). Written informed consent forms were obtained from each patient upon study entry. The study was conducted in adherence to the Good Clinical Practice guidelines and with regulatory requirements consistent with the Declaration of Helsinki. The study is registered in the Netherlands Trial Register, number NTR3112.

\section{MEASUREMENTS}

Baseline characteristics included: age, sex, disease duration, medication use, and rheumatoid factor (RF) and anti-cyclic citrullinated peptide (anti-CCP) antibody status. Patients were evaluated at baseline and at least once every three months thereafter up to 1 year by the attending rheumatologist and rheumatology nurse, in accordance with current Dutch treatment guidelines for RA. Clinical measurements which are part of standard rheumatology care were performed at every visit and included the tender joint count in 
28 joints, the swollen joint count in 28 joints, the erythrocyte sedimentation rate (ESR), C-reactive protein (CRP) and physician and patient global assessment on a 0-100 visual analog scale (VAS). Additionally, physician-reported flares and changes in medication were recorded at each scheduled or unscheduled visit.

The following patient reported outcomes were also administered at each study visit. Fatigue was assessed using the Bristol RA Fatigue Multi-Dimensional Questionnaire and 0-10 numerical rating scales assessing fatigue severity and effect of fatigue on daily functioning. Patient reported wellbeing, disease activity and pain were assessed using 0-100 VAS. The Health Assessment Questionnaire disability index (HAQ-DI) was administered to evaluate disability. Finally the SF-36 was administered to assess health-related quality of life. We used the role physical, role emotional and social functioning scale to assess participation. The bodily pain, physical functioning and vitality scales were used to assess pain, disability and fatigue, respectively. Clinical disease activity index (CDAl), simplified disease activity index (SDAI) and DAS28-ESR scores were calculated using their respective standard scoring algorithms ${ }^{(4)}$.

\section{STATISTICAL ANALYSIS}

For this post-hoc analysis, data from 462 patients randomized to stopping TNFi treatment were used. Standardized effect size (ES) statistics with pooled SDs were obtained to compare the ability of various measures to discriminate between patients with and without flare at the 3-months assessment (T2). The first flare anchor we examined was physician-reported flare at T2. Medication escalation was also evaluated as an anchor of flare, defined as starting or increasing any biological or non-biological DMARD (including glucocorticoids) at T2.

Responsiveness was evaluated by comparing standardized change scores (SCS), again with pooled standard deviation from baseline to T2. The magnitude of ES and SCS was interpreted according to the guidelines provided by Cohen (i.e. trivial $<0.20$, small $\geq 0.20$, moderate $\geq 0.50$ and large $\geq 0.80$ ) ${ }^{(22)}$. The utility of individual measures to predict flare status at T2 was investigated next using univariable logistic regression analysis. For each domain, the outcome measure with the largest ES in the analysis of discriminant validity was selected to represent that domain. Predictive strength was quantified using $R^{2}$, as an effect size estimator. Guidelines for interpreting $R^{2}$ were again derived from Cohen: small $\geq 0.01$, moderate $\geq 0.09$ and large $\geq 0.20{ }^{(22)}$. The incremental validity of outcome domains not included in the SDAI was evaluated using multivariable hierarchical logistic regression analysis with flare status at $\mathrm{T} 2$ as the dependent variable. CDAl was entered as a first block and individual outcome measures were entered as a second block. Incremental validity 
was evaluated using $\Delta R^{2}$.

Multiple imputation by chained equationswas performed to replace missing values. The imputation modelincluded the dependent variable; physician-reportedflare status (yes/ no),the evaluated outcome measures as well as age, follow up time and total number of flares during the total study period. 20 datasets with imputed plausible values were obtained, with 200 iterations between data sets. Rubin's rules were employed to obtain pooled parameter estimates and their associated standard errors. Pooled analyses will be reported throughout thepaper.

\section{RESULTS}

Baseline characteristics are presented in table 1. The sample was characterized by established disease, with high disease duration, and $62 \%$ of patients with erosive disease. In accordance with the inclusion criteria, disease activity according to DAS28 was low at baseline, as was physical disability according to HAQ-DI.

Table 1. Patient characteristics

\begin{tabular}{ll}
\hline & Stop group (N=462) \\
\hline Age in years (SD) & $59.5(10.5)$ \\
Disease duration in years & $12.0(8.9)$ \\
Sex, N (\%) female & $181(66 \%)$ \\
DAS28 (SD) & $1.90(0.8)$ \\
HAQ-DI (SD) & $0.6(0.6)$ \\
Erosive disease N (\%) & $294(62.6 \%)$ \\
Rheumafactor positive N (\%) & $316(67.2 \%)$ \\
\hline
\end{tabular}

DAS28 = 28 joint-Disease Activity Score; HAQ-DI = Health Assessment Questionnaire Disability Index

\section{DISCRIMINANT VALIDITY}

While $39.4 \%$ of patients had a medication escalation at T2, only $18.7 \%$ of patients were reported to have a clinical flare at T2 by their rheumatologist. A consistently greater contrast in scores (i.e. ES of greater magnitude) was observed for clinical flare compared with medication escalationacross all measures. The clinical composite scores performed best and interchangeably, followed by pain and physician global assessment. Bodily pain assessed using the SF-36 was consistently the best performing individual measure with ES of moderate magnitude, followed by physician global assessment and patient global assessment of disease activity and wellbeing. Measures of physical aspects of patient 
reported health and fatigue had ES of small to moderate magnitude. Measures of emotional wellbeing consistently had ES of trivial magnitude (Figure 1).

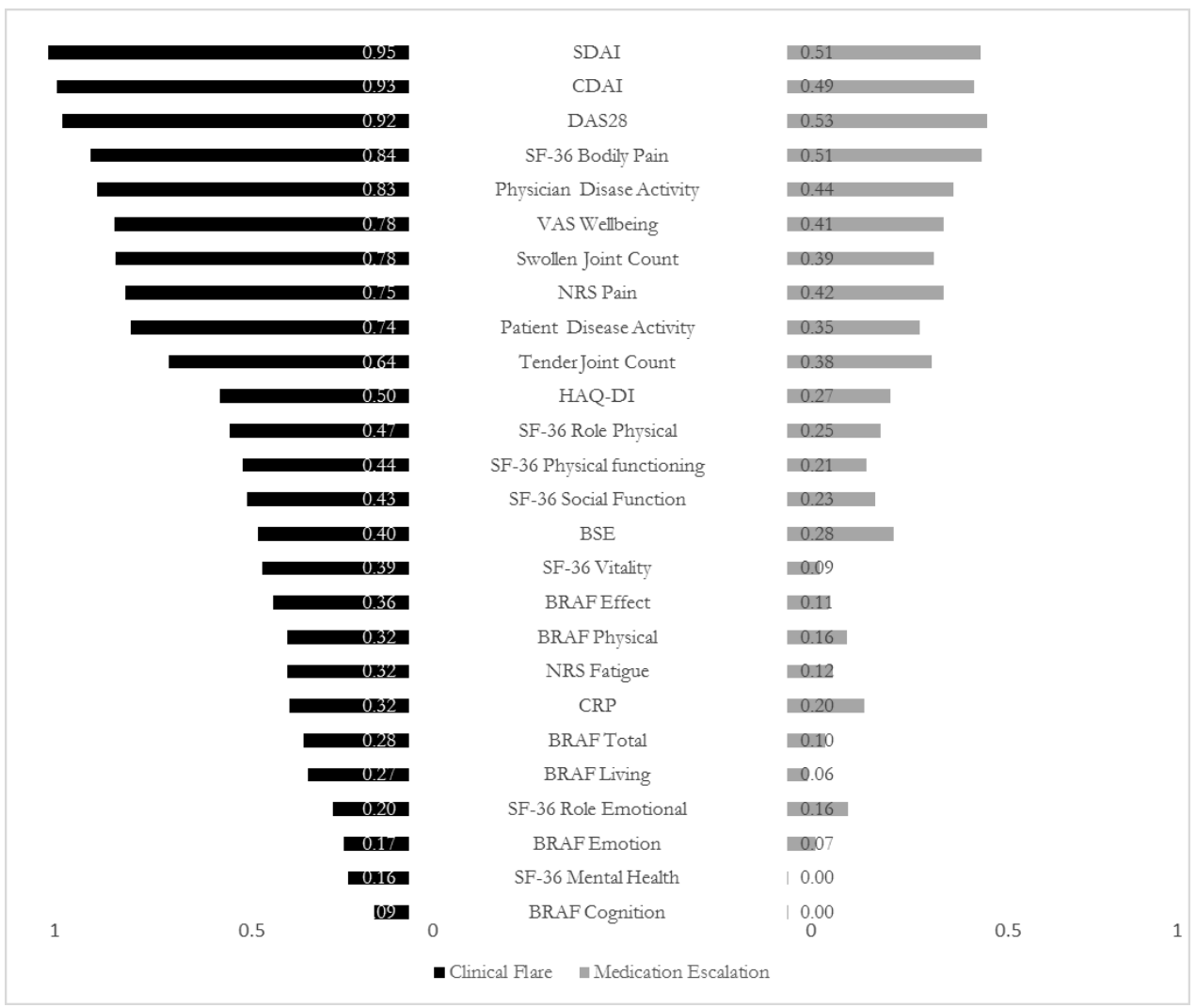

\section{Figure 1. Discriminative ability}

Bar charts represent Effect size statistics DAS28 = 28 joint-Disease Activity Score; ESR =erythrocyte sedimentation rate; $\mathrm{CDAl}=$ Clinical Disease Activity index; SDAI = SimplifiedDisease Activity index; SF-36 =Short Form Health Survey 36; HAQ-DI = Health Assessment Questionnaire Disability Index ; VAS = Visual Analogue Scale; CRP = C-reactive protein; NRS = Numerical Rating Scale; BRAF = Bristol RA Fatigue Scale. 


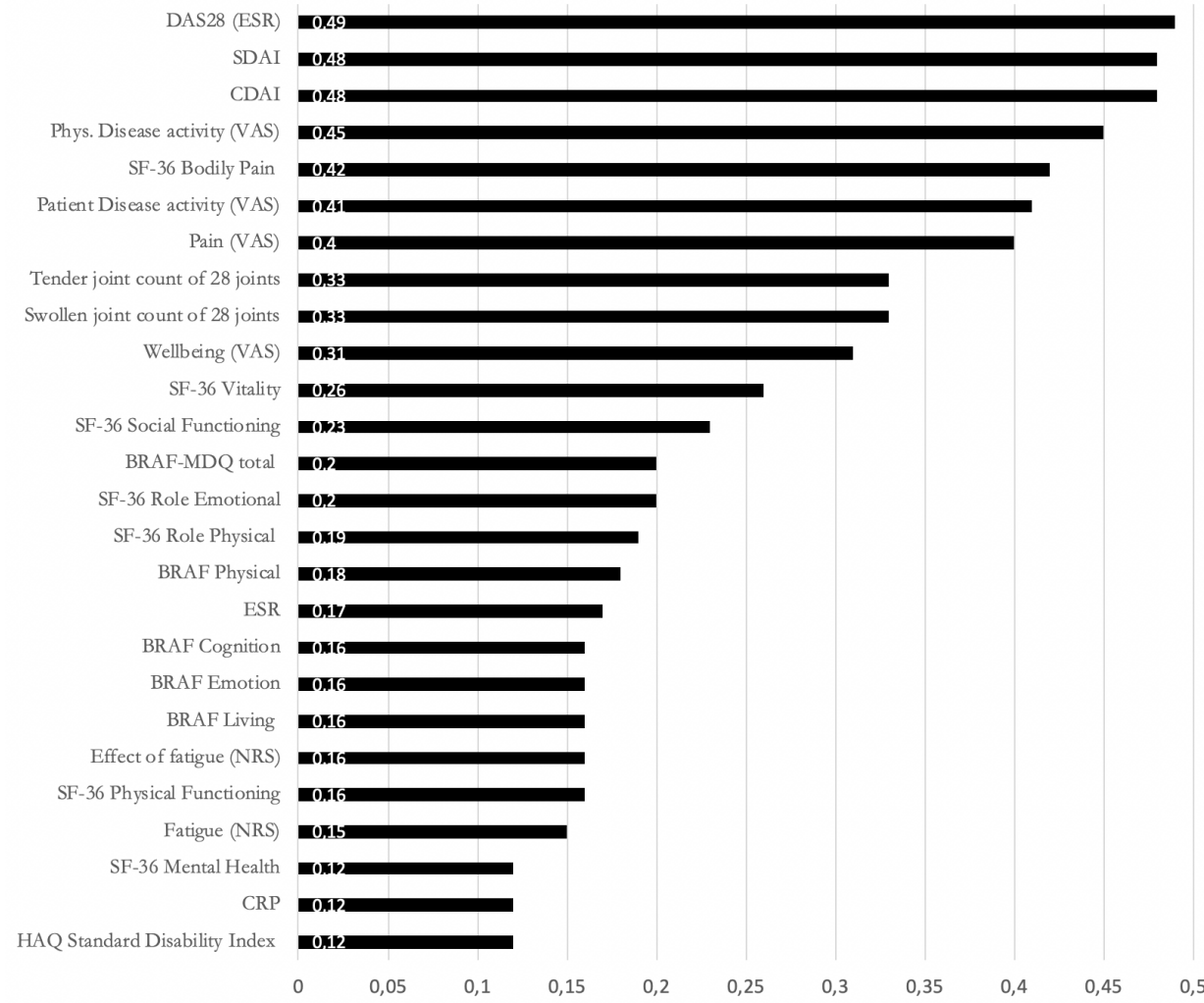

Figure 2. Standardized change scores in POET stop group

Bar charts represent Effect size statistics DAS28 = 28 joint-Disease Activity Score; ESR =erythrocyte sedimentation rate; $\mathrm{CDAI}=$ Clinical Disease Activity index; SDAI = SimplifiedDisease Activity index; SF-36 =Short Form Health Survey 36; HAQ-DI = Health Assessment Questionnaire Disability Index; VAS = Visual Analogue Scale; CRP = C-reactive protein; NRS = Numerical Rating Scale; BRAF = Bristol RA Fatigue Scale.

\section{RESPONSIVENESS}

A moderate increase in disease activity according to all three disease activity indices (SCS $\approx 0.50$ ) was observed for the stop group. Individual DAS28 components showed SCS of trivial (ESR) to small magnitude (TJC, SJC \& wellbeing). Physician global assessment of disease activity was the only individual measure with SCS of moderate magnitude. Patient assessment of disease activity and both measures of pain had SCS of small magnitude, but outperformed each of the individual DAS28 components, while the SF-36 vitality and social function scales had SCS of small magnitude and smaller than the DAS28 components. Each of the remaining measures hada SCS of trivial magnitude (Figure 2). 


\section{INCREMENTAL VALIDITY}

In univariable analysis, all outcomes except emotional wellbeing were significantly associated with clinical flare status and all outcomes except emotional wellbeing and fatigue were significantly associated with medication escalation at T2. Of the measures included in SDAI, CRP was consistently most weakly associated with flare status in univariable analysis, while Physician Global performed best. Of the measures not included in SDAI, Pain was consistently most strongly associated with 3 months. Pain and physician global assessment were performed best overall. According to the analysisin the binary logistic regression models with only $\mathrm{SDAl}, R^{2}$ was of small magnitude for medication escalation $\left(R^{2}=0.09\right)$ and large magnitude $\left(R^{2}=0.26\right)$ for clinical flare. In table 2 it can be seen that only pain contributed significantly to the classificationof flare status beyond the SDAI. However the magnitude of its independenteffect was small.

Table 2. Univariable and multivariable predictors of flare status

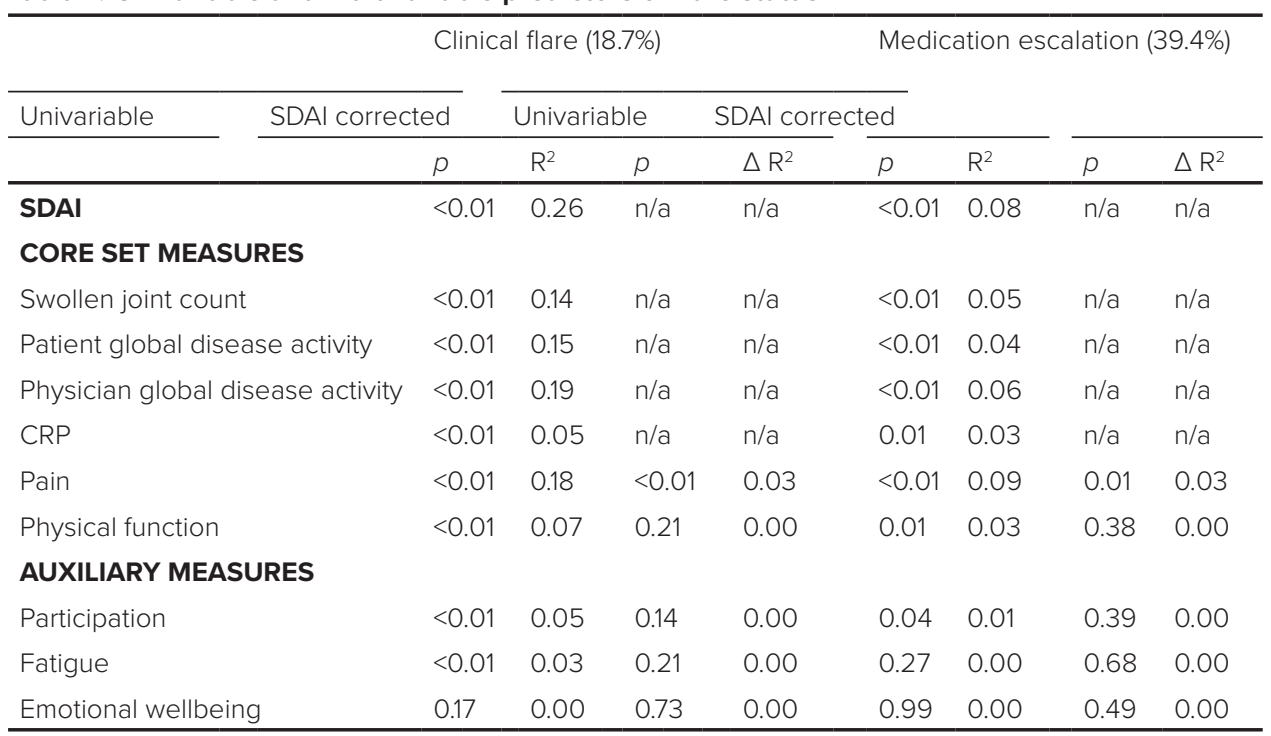




\section{DISCUSSION}

In the present study, the performance for assessing disease exacerbation was compared between several validated disease activity indices and individual measures that either assess one of the core set variables or one of thepatient-reported outcome domains that have recently been proposed as outcomes for the assessment of flare in RA.

Overall, the composite disease activity indices outperformed individual measures across the various comparisons. This was unsurprising since standardized change scores of individual measures are affected more by measurement error compared with composite scores, sincerandom error tends to cancel itself out when information from multiple measures is combined. Nevertheless, the disease activity indices proved to be noticeably more efficient indicators of disease exacerbation, compared with most individual measures which further supports the practice of usingcomposite tools for assessing disease activity in RA.

Interestingly, despite their different constituent measures and scoring protocols, the CDAI, SDAI and DAS28 performe dequivalently as measures of disease exacerbation in the present study. Similar sensitivity to improvement was observed as wellfor these three tools in two previous studies ${ }^{(23,24)}$ and no clear differences in other measurement properties were observed in a comparative systematic review ${ }^{25}$. When measuring disease activity exacerbations, it seems therefore that the main advantages of DAS28 are that that it has most frequently been used in previous studies, providing a richer frame of reference for the interpretation of study outcomes against historical results and that it is currently the only clinical composite score for which validated flare criteria are available ${ }^{(26)}$. However, scoring ismore complex than for SDAl and CDAl due to differential weighting of individual components. Importantly also, acute-phase reactants were shown in this study to provide little additional information on disease activity exacerbation and were among the weakest univariable predictors of flare status. Since there is usually a delay in the availability of laboratory results, the inclusion of acute phase reactants inboth the SDAI and DAS28may create a logistical barrier to obtaining disease activity scores in real time, for apparently redundant information from a statistical point of view.

Physician and patient global assessment of disease activity and pain assessed using eithervisual analogue scale or the SF-36 bodily pain scale were the most sensitive individual measures of increased disease activity in the POET study. It is worth mentioning that changes in the amount of pain a patient reports was more predictive than other variables measured in this study of treatment decisions (medication escalation) and physician-reported flare. Pain intensity was alsothe only outcome domain that provided 
unique information beyond the information provided by the SDAl in the analysis of incremental validity, even though pain is indirectly represented in both the tender joint count and, very likely, patient global, which were both controlled for in the analysis. Previous studies have repeatedly established that pain is the most importanttreatment priority for patients, throughout the disease course ${ }^{(27)}$. These results suggest thatmore comprehensive evaluation of overall disease status of a patient could be obtained using a clinical composite score that includes pain.

The overall performance ranking of specific measures in our study corresponds largely to that observed in previous studies that have compared the ability of different measures to assess disease improvement. Only the poor performance of physical function contrasts with previous studies where it hasconsistently been foundto be one of the best performing indicators of treatment benefits ${ }^{(7-12)}$ and increased disease activity in an observational study ${ }^{(28)}$. These contrasting results arelikely explained bythe high average disease duration and the high prevalence of patients with erosive disease at baseline in POET, which previous studies have found to be associated with decreased responsiveness when measuring physical function in patients with RA ${ }^{(29)}$. It is commonly believed that while inflammatory disease activity is the most important determinant of physical function in RA, later in the disease course structural damage increasingly contributes to disability, effectively lowering the ceiling on the amount of improvement that can be realized ${ }^{(30)}$. Our results provide further support for this notion and suggest that inferences regarding disease exacerbations from (lack of) change in physical function scores should be made with caution in populations with longstanding disease.

Little evidence was found in the present study to support an extended set of outcomes for measuring flares. PRO's other than pain and patient global assessment were found to be weakly sensitive to disease flareand did not provide incremental information above and beyond the SDAI when predicting flare status. These results suggest that the addition of fatigue, emotional well being or participation to a composite score may not contribute much to its reliability or predictive validity for measuring flares or exacerbated disease. Previous studies also found limited responsiveness for measures of participation and emotional wellbeing, while the performance of fatigue has been mixed ${ }^{(6-11)}$. An explanation for this may be that participation restrictions and emotional wellbeing are complex, integrated domains that may be determined by disease activity as well as comorbidities, and personal and environmental factors.

Strong points of this study arethat the POETstudy was designed to evaluate exacerbation of RA resulting from TNFi discontinuation in patients with longstanding stable low disease 
activity and that physician-rated flares were assessed per protocol, while previous studies are post-hoc analyses of subsets of deteriorated patients from studies originally designed to evaluate patients expected to improve (e.g. clinical trials) or remain stable (observational studies) ${ }^{(28,31-34)}$. A limitation of the study is that there was relatively sparse variability of scores for some measures (e.g. swollen joint count) due to the homogenous, low level of disease activity at start of the study. Different results might have been achieved in patients with more severe disease at baseline. However, practical measurement settings in which disease worsening is to be assessed, most likely involve patients starting in a state of low disease activity (e.g. tapering of medication in daily clinical practice or clinical trials).

In summary, the results of this study support the use of traditional disease activity measures and composite indices for assessing flare or disease exacerbation. Pain and physicianand patient-reported global assessment of disease activity were the best performing individual measures, while the composite DAS28, CDAl and SDAl performed equivalently as measures of exacerbation. Patient-reported measures assessing the domains of participation, fatigue and emotional functioning performed worst.Based on these findings we recommend that assessment of disease flares should proceed using, preferably combined, core set measures, particularly pain intensity. 


\section{REFERENCES}

1. Pincus T. The American College of Rheumatology (ACR) Core Data Set and derivative "patient only" indices to assess rheumatoid arthritis. Clin Exp Rheumatol 23:S109-13.

2. Boers $M$, Tugwell $P$, Felson DT, Riel PL van, Kirwan JR, Edmonds JP, et al. World Health Organization and International League of Associations for Rheumatology core endpoints for symptom modifying antirheumatic drugs in rheumatoid arthritis clinical trials. J Rheumatol Supp/ 1994;41:86-9

3. Felson DT, Anderson JJ, Boers $M$, Bombardier C, Chernoff M, Fried B, et al. The American College of Rheumatology preliminary core set of disease activity measures for rheumatoid arthritis clinical trials. The Committee on Outcome Measures in Rheumatoid Arthritis Clinical Trials. Arthritis Rheum 1993;36:729-40.

4. Aletaha D, Smolen J. The Simplified Disease Activity Index (SDAI) and the Clinical Disease Activity Index (CDAI): a review of their usefulness and validity in rheumatoid arthritis. Clin Exp Rheumatol 23:S100-8

5. Gestel AM van, Prevoo ML, 't Hof MA van, Rijswijk MH van, Putte LB van de, Riel PL van. Development and validation of the European League Against Rheumatism response criteria for rheumatoid arthritis. Comparison with the preliminary American College of Rheumatology and the World Health Organization/International League Against Rheumatism Cri. Arthritis Rheum
1996;39:34-40.

6. Aletaha D, Landewe $\mathrm{R}$, Karonitsch $\mathrm{T}$, Bathon J, Boers M, Bombardier C, et al. Reporting disease activity in clinical trials of patients with rheumatoid arthritis: EULAR/ ACR collaborative recommendations. Ann Rheum Dis 2008;67:1360-4

7. Buchbinder R, Bombardier C, Yeung M, Tugwell P. Which outcome measures should be used in rheumatoid arthritis clinical trials? Clinical and quality-of-life measures' responsiveness to treatment in a randomized controlled trial. Arthritis Rheum 1995;38:1568-80

8. Anderson JJ, Chernoff MC. Sensitivity to change of rheumatoid arthritis clinical trial outcome measures. J Rheumatol 1993;20:535-7.

9. Verhoeven AC, Boers M, Linden S van Der. Responsiveness of the core set, response criteria, and utilities in early rheumatoid arthritis. Ann Rheum Dis 2000;59:966-74.

10. Russell AS, Conner-Spady B, Mintz A, Maksymowych WP. The responsiveness of generic health status measures as assessed in patients with rheumatoid arthritis receiving infliximab. J Rheumatol 2003;30:941-7.

11. Tugwell P, Wells G, Strand V, Maetzel A, Bombardier C, Crawford B, et al. Clinical improvement as reflected in measures of function and health-related quality of life following treatment with leflunomide compared with methotrexate in patients with rheumatoid arthritis: sensitivity and relative efficiency to detect a treatment e. 
Arthritis Rheum 2000;43:506-14.

12. Cohen SB, Strand V, Aguilar D, Ofman JJ. Patient- versus physician-reported outcomes in rheumatoid arthritis patients treated with recombinant interleukin-1 receptor antagonist (anakinra) therapy. Rheumatology (Oxford) 2004;43:704-11.

13. Guyatt GH, Deyo RA, Charlson M, Levine MN, Mitchell A. Responsiveness and validity in health status measurement: a clarification. J Clin Epidemio/ 1989;42:4038.

14. Neogi T, Xie H, Felson DT. Relative responsiveness of physician/assessorderived and patient-derived core set measures in rheumatoid arthritis trials. $J$ Rheumatol 2008;35:757-62.

15. Broeder AA den, Maas A van der, Bemt BJF van den. Dose de-escalation strategies and role of therapeutic drug monitoring of biologics in RA. Rheumatology (Oxford) 2010;49:1801-3.

16. Alten R, Pohl C, Choy EH, Christensen R, Furst DE, Hewlett SE, et al. Developing a construct to evaluate flares in rheumatoid arthritis: a conceptual report of the OMERACT RA Flare Definition Working Group. J Rheumatol 2011;38:1745-50.

17. BINGHAM CO, POHL C, WOODWORTH TG, HEWLETT SE, MAY JE, RAHMAN MU, et al. Developing a Standardized Definition for Disease \&quot;Flare\&quot; in Rheumatoid Arthritis (OMERACT 9 Special Interest Group). J Rheumatol 2009;36:2335-2341.

18. Bartlett SJ, Bykerk VP, Cooksey R, Choy EH, Alten R, Christensen R, et al. Feasibility and
Domain Validation of Rheumatoid Arthritis (RA) Flare Core Domain Set: Report of the OMERACT 2014 RA Flare Group Plenary. J Rheumatol 2015.

19. Berthelot J-M, Bandt $M$ De, Morel J, Benatig F, Constantin A, Gaudin P, et al. A tool to identify recent or present rheumatoid arthritis flare from both patient and physician perspectives: the "FLARE" instrument. Ann Rheum Dis 2012;71:1110-6.

20. Arnett FC, Edworthy SM, Bloch DA, McShane DJ, Fries JF, Cooper NS, et al. The American Rheumatism Association 1987 revised criteria for the classification of rheumatoid arthritis. Arthritis Rheum 1988;31:315-24.

21. Prevoo ML, 't Hof $M A$ van, Kuper $\mathrm{HH}$, Leeuwen MA van, Putte LB van de, Riel PL van. Modified disease activity scores that include twenty-eight-joint counts. Development and validation in a prospective longitudinal study of patients with rheumatoid arthritis. Arthritis Rheum 1995;38:44-8.

22. Cohen J. A power primer. Psychol Bull 1992.

23. Aletaha D, Nell VPK, Stamm T, Uffmann M, Pflugbeil S, Machold K, et al. Acute phase reactants add little to composite disease activity indices for rheumatoid arthritis: validation of a clinical activity score. Arthritis Res Ther 2005;7:R796-806.

24. Ranganath VK, Yoon J, Khanna D, Park GS, Furst DE, Elashoff DA, et al. Comparison of composite measures of disease activity in an early seropositive rheumatoid arthritis 
cohort. Ann Rheum Dis 2007;66:1633-40.

25. Gaujoux-Viala C, Mouterde G, Baillet A, Claudepierre P, Fautrel B, Loët X Le, et al. Evaluating disease activity in rheumatoid arthritis: which composite index is best? A systematic literature analysis of studies comparing the psychometric properties of the DAS, DAS28, SDAI and CDAI. Joint Bone Spine 2012;79:149-55.

26. Maas A van der, Lie E, Christensen R, Choy E, Man YA de, Riel P van, et al. Construct and criterion validity of several proposed DAS28-based rheumatoid arthritis flare criteria: an OMERACT cohort validation study. Ann Rheum Dis 2013;72:1800-5.

27. Klooster PM ten, Veehof MM, Taal E, Riel PLCM van, Laar MAFJ van de. Changes in priorities for improvement in patients with rheumatoid arthritis during 1 year of anti-tumour necrosis factor treatment. Ann Rheum Dis 2007;66:1485-90.

28. Lie E, Woodworth TG, Christensen R, Kvien TK, Bykerk V, Furst DE, et al. Validation of OMERACT preliminary rheumatoid arthritis flare domains in the NOR-DMARD study. Ann Rheum Dis 2014;73:1781-7.

29. Ward MM. Interpreting measurements of physical function in clinical trials. Ann Rheum Dis 2007;66 Suppl 3:iii32-4.

30. Drossaers-Bakker KW, Buck M de, Zeben D van, Zwinderman AH, Breedveld FC, Hazes JM. Long-term course and outcome of functional capacity in rheumatoid arthritis: the effect of disease activity and radiologic damage over time. Arthritis Rheum 1999;42:1854-60.
31. Hays RD, Spritzer KL, Fries JF, Krishnan E. Responsiveness and minimally important difference for the patient-reported outcomes measurement information system (PROMIS) 20-item physical functioning short form in a prospective observational study of rheumatoid arthritis. Ann Rheum Dis 2015;74:104-7.

32. Linde L, Sørensen J, Ostergaard $M$, Hørslev-Petersen K, Hetland ML. Healthrelated quality of life: validity, reliability, and responsiveness of SF-36, 15D, EQ-5D [corrected] RAQoL, and $H A Q$ in patients with rheumatoid arthritis. J Rheumatol 2008;35:1528-37.

33. Fransen J, Häuselmann $H$, Michel BA, Caravatti M, Stucki G. Responsiveness of the self-assessed rheumatoid arthritis disease activity index to a flare of disease activity. Arthritis Rheum 2001;44:53-60.

34. Taal E, Rasker JJ, Riemsma RP. Sensitivity to change of AIMS2 and AIMS2-SF components in comparison to $\mathrm{M}-\mathrm{HAQ}$ and VAS-pain. Ann Rheum Dis 2004;63:1655-8. 

Chapter 8

General discussion 
With the turn of the centurty, the treatment of patients with Rheumatoid Arthritis (RA) started to change from care to a situation where cure has become a realistic option. Basis for this change is the development and introduction of inhibitors of Tumor Necrosis Factor (TNFi) and other targets thereafter. In hind sight, the efficacy of these new class of pharmacological agents: referred to as biologicals, proofed to be better than expected, changing the perspective of many RA patients and patients suffering from other autoimmune diseases. Typically during the development and introduction of TNFi the attention was focused on efficacy and safety. Drug tapering or even more stopping, in case of favorable efficacy, was not on our minds, The paradigm that auto-immune diseases, like RA, are chronic conditions that need live long treatments, and in best cases progression could be retarded, continued to dominate the thinking of healthprofessionals and patients. We were confronted with the need to stop or discontinue TNFi, in cases where the anticipate increased risk for infections became reality ${ }^{(1)}$. Guidelines to decrease the infectious risk related to the inhibition of TNF were developed and successfully implemented (biobadaser TNF en TBC) ${ }^{(2)}$. Although scarcely evaluated, stopping TNFi in case of infection or increased risk for infection (elective surgery) the clinical impression was that flares occurred following stopping where restarting TNFi resulted in quick regained response ${ }^{(3)}$.

Studies on remission induction ${ }^{(4)}$, later referred to as treating to the target of remission $(\mathrm{t} 2 \mathrm{~T})^{(5)}$, was a direct consequence of the impressive clincal efficacy of the introduction on biologicals ic TNFi. The high percentage of RA patients in real life studies, that were able to reach stable remission by using the therapeutic options in combination with the increasing drug costs and concern on lifelong treatments paved the way for studies on drug tapering or stopping TNFi in case of excellent clinical response (remission of low disease activity). Although scientific arguments, like immunogenicity ${ }^{\left({ }^{6}\right)}$ against drug tapering outweigh the arguments against immediate stopping, psychological arguments form patients as well as health professionals lead the majority of studies started with drug tapering strategies. The majority of studies are investigator initiated and for obvious reasons small and subjected to methodological limitations. The POET study, that is the basis for my thesis, was initiated by Dutch Rheumatologists and Health Policy Makers. The combined Dutch effort resulted in one of the largest studies on the subject of dose optimalization of biologicals in RA in this case by stopping. Although this study also has its limitations ic the open label design and the pragmatic randomized design, the impressive number of participating patients enabled us to address not only the primary study question but also to explore additional questions like mechanisms involved and impact for patients and society.

In the first chapter of my thesis I discussed existing information on stopping and tapering of biologicals in RA as well as a theoretical framework potentially explaining underlying mechanisms. 


\section{CHAPTER 2}

This chapter is the primary and most important publication of the POET study, it was my privilege, on behalf of the POET team to show that stopping TNFi is possible in $48 \%$ of RA patients in sustained remission or LDA. However, this strategy results in more than threefold increase signs and symptoms of increased disease activity (flare) within 12 months as compared to patients who continued TNFi. We also showed that mean disease activity in the stop group was significantly increased throughout the follow-up period but in most patients it remained below the threshold for moderate disease activity. With regard to the fact that different flare definitions are available, we used sensitivity analyses using other definitions of flare, which showed comparable results. Our results confirm findings of several, mostly smaller studies:

Preceding studies on stopping TNFi in RA demonstrated a wide range of flare risk of 18-88\%. Difference in results is possibly explained by heterogeneity in RA population, definitions of flare, disease activity at inclusion, and use of concomitant cSDMARDs. RCTs reporting the results of stopping specific TNFi report the following results. The US Corrona registry reported that $73.4 \%$ of RA patients experienced no flare for more than 12 months after stopping their first TNFi ${ }^{(7)}$. The EMPIRE trial, PRESERVE trial, and DOSERA study reported that respectively $57.7 \%, 42,6 \%$ and $13 \%$ of RA patients successful stopping etanercept (8,9,10); The OPTIMA trial, HONOR and BRIGHT studies reported that respectively 56\%, 58\% and $18 \%$, of RA patients remained in remission or LDA after stopping adalimumab ${ }^{(1,1,1,13)}$. Stopping infliximab by Quinn et al, the BeSt study (after falling DMARD) and RRR reported persistent LDA of remission in $70 \%, 25 \%$ and $50 \%$ respectievely ${ }^{(14,15,16)}$. Finaly a small study on stopping certolizumab pegol reported absence of any success ${ }^{(17)}$.

In our POET study, in case of flare after stopping TNFi, we suggested restarting TNFi. The observed data show that treatment success could be realized in the majority of patients (83.1\% success after restarting TNFi, most of the time within 12 weeks). Moreover, there were no notable (serious) adverse events after restarting TNFi.

Finely we noticed more hospitalization in the stop group than continuation group, but most of them were not related to the stopping TNFi. Reasons for hospitalization that were observed are infections, elective surgery, malignancies or fractures. We concluded that stopping TNFi in RA patients and restarting TNFi in case of flare seem to be safe.

In addition to the stopping TNFi, there are multiple stategies for dose reduction also referred to as dose optimalization. For obvious reasons the variability to do so exceeds the options for direct stopping like we did in the POET study. The PRESERVA-, DOSERA- and DRESS study used different strategies to taper than STRASS- en RETRO study ${ }^{(9,10,18,19,20)}$. In 
general these studies showed that less flare occured during dose tapering compared with stopping TNFi, but overall comparable long term effects for patients were seen.

The POET study only addressed stopping TNFi. Other studies examined the effects of TNFi dose reduction. Some trials compared etanercept reduction versus stopping or continuation and concluded that reduced dosing generally resulted in an increased disease activity and or flares. Although the risk of flares during doses reduction was not to the extend of immediate stopping, the cumulative risk of al consecutive reductions is comparable with the flare risk of stopping immediately ${ }^{(21-24)}$. More recently, in the DRESS study showed that disease activity-guided dose reduction of adalimumab or etanercept was not inferior to continuation with respect to flares ${ }^{(18)}$, comparable to our findings.

\section{CHAPTER 3}

In this chapter we search for predictors for successful discontinuation of TNFi. This chapter serves the theoretical frame work discussed in chapter one. Our analyses showed that $70 \%$ of RA patients in sustained remission or stable LDA, who used a TNFi of the antibody class, with a relative short disease duration of $<10$ years and a MBDA score $\leq 44$ were most likely be able to stop their TNFi without a subsequent flare. In contrast to patients on a TNFi receptor antagonist, with a disease duration of $\geq 10$ years and a MBDA score $>44$ who $80 \%$ needed to restart TNFi because of experiencing a flare.

The POET observation that relative short disease duration was related to stopping without flare was in line with the the study of Kavanaugh et al., it was reported that successful stopping bDMARDs in RA patients with early disease ${ }^{(25)}$. However Kuiper et al. showed that early RA patients had no lower flare rate than stablished RA after stopping bDMARDs (26). The latter might be explained by the small number of patients.

In our study, RF positivity, non-smoking and normal BMI were not found to be predictors of successful discontinuation. In contrast with the data from Corrona registry and RRR study who reported these patients characteristics as a predictor for successful TNFi discontinuation ${ }^{(7,16)}$. A possible explanation for the absence of a relation with RF and aCCP might be the high percentage of double positive patients in our study.

Also signs and symptoms related to residual disease activity at the time of discontinuation seems to be a predictor for flare after stopping TNFi ${ }^{(13,16,27)}$. the association with a higher MBDA score at baseline in our POET study is in line with this hypothesis.

We demonstrated that the type of TNFi could be a predictor. Our analyses showed that 
patients using TNF antibodies (mostly adalimumab) were significantly more often able to successfully discontinue their TNFi than patients who had been using TNF receptor antagonists (mostly etanercept). TNF antibodies seems to induced prolonged disease control due to langer half-life, lysing surface TNF expressing blood cells and block TNF interaction with cell surface TNF receptors. This is in line with the recent publication of $\mathrm{M}$ Hashimoto et al. ${ }^{(28)}$.

Taken the information of the analysis presented in chapter 3 together with the theoretical framework presented in chapter 1 we found support for a role for the window of opportunity, residual disease activity and mode of TNF-inhibitor ic antibodies as compared to receptor antagonist. Obviously these post hoc analysis can not confirm the theory but are in line with the underlying hypothesis.

\section{CHAPTER 4}

The multi-biomarker disease activity (MBDA) blood test measures 12 serum proteins to produce a score that has been clinically validated as a measure of disease activity in patients with RA ${ }^{(29-31)}$. MBDA scores have been shown to reflect current clinical disease activity and changes in disease activity over time, including treatment responses in RA patients treated with $\mathrm{TNFi}^{(32)}$. In this chapter we focused on the MBDA score as a predictor of flare after discontinuation of TNFi treatment. We used the data from patients who randomized to the stop group. In this group, the baseline serum samples were available in majority of patients to measure MBDA scores. We showed that patients with a high MBDA score at the time of TNFi discontinuation had significantly more flare in follow-up during 12 months. The data suggested that a high MBDA score, although the patient is in remission or a state of LDA, could be a sign of residual disease activity. Several previous studies have found elevated MBDA score in RA patients with remission of LDA ${ }^{(33-36)}$. We reported for the first time, to the best of our knowledge, the value of the MBDA score as a predictor for flare in RA patients who discontinued TNFi treatment.

Considering the three different predictor criteria for relapse (TNFi restart, medication escalation, and physician-reported flare), in our study a high MBDA score was an independent predictor of all three potential predictors but it did not remain associated when adjusting for all other potential predictors, including DAS28 score.

Although the heterogenicity of the disease rheumatoid arthritis and consequently the difficulty of assessing disease activity is well known, laboratory based methods like the MBDA, up to present, are not able to overcome the limitations of composite disease activity scores. The results of the POET study do confirm that there is a relation between 
RA disease activity and MBDA scores but this weak relation probably does not allow the costs of the test ${ }^{(37)}$.

\section{CHAPTER 5}

Apart from the biomedical perspective of the disease the perspective of the patient is by intention reflecting the consequences of the disease for the health status of the patient. Therefore, in the POET study we addressed in addition to the outcomes in the biomedical perspective the impact for the patient as wells. We demonstrated that stopping TNFi had a significant but short-term negative impact on patient-reported outcomes (PROs) such as physical and mental health status, health utility, pain, disability and fatigue.

There are only a few studies in which the effect of stopping TNFi on burden of disease were analyzed. The randomized controlled trial PRESERVE study showed after 12 months a better score on general health, pain, disability, health utility, sleep, and fatigue in patients who continued etanercept plus methotrexate than patients who stopped etanercept and used methotrexate only ${ }^{(9)}$. In addition, the observational RRR study showed that after one-year the mean HAQ-DI scores were significantly lower in patients who remained in remission after stopping infliximab versus those that flared ${ }^{(16)}$. This result was not supported by OPTIMA - and ADMIRE-studies ${ }^{(11,38)}$. Also the result of the open-label nonrandomized HONOR study did not show a significant difference HAQ-DI scores after one year adalimumab discontinuation between patients that sustain in remission and patients who flared ${ }^{(12)}$. In a very small observational study of Brocq et al, in 21 patients, they did not found significant differences in one-year HAQ-DI scores between stopping of TNFi (39).

In general the negative biomedical effects of stopping TNFi in RA patients seem to be reflected in unfavorable Patient Reported Outcomes. However in line with the handling with care design of the different studies the negative outcomes are small and of short duration not impacting the long-term health of patients trying to stop or tapper their expensive treatments.

\section{CHAPTER 6}

Since drug cost was one of the important arguments for the discussion on stopping biologicals as a cost analysis of stopping versus continuing TNFi in case of good disease control ( remission or LDA, as defined by DA28) was almost mandatory. We demonstrated 
that stopping TNFi in this group of RA patients can save cost but comes with a small loss of QALY. The mean saved cost was $€ 368,269$ per QALY lost. We showed that, except for the first follow-up point after baseline, the mean health utility did not significantly differ over time between patients in the stop and continuation groups. We suggest that stopping TNFi in well controlled RA patients comes with considerable costs saved and temporarily limited loss of quality of life.

In the, also Dutch, tapering study, the authors showed that guided down titration of TNFi could save $€ 390,493$ for each QALY lost ${ }^{(40)}$ remarkably compared with discontinuation of TNFi in our stop study. In another cost analysis based on the STRASS study, the authors reported that step-down strategy results in saving 53,417 euros per QALY lost ${ }^{(41)}$. In the PRESERVE study, in which dose reduction was studied in the case of remission, Kobelt et al. reported the cost per QALY gained for the half-dose strategy versus stopping etanercept varies between $€ 14,000$ and $€ 29,000$, depending on the time frame. The author suggested that a dose reduction is the most advantageous strategy in patients with moderate RA, although cost advantage of stopping TNFi was not separately reported (42). Some studies only reported dug cost saved: Van der Maas et al. reported a mean cost reduction of 3474 euros per patient due to infliximab tapering (43). A comparable result has been shown by Murphy et al. in a cohort of 79 patients with rheumatoid arthritis, psoriatic arthritis, and ankylosing spondylitis. They reported a mean saving of 3800 euros per patient per year after dose reduction of etanercept or adalimumab (44).

\section{CHAPTER 7}

In this chapter we did a post-hoc analyses of data to identify the most reliable measurement instruments for disease exacerbation. We showed that the composite disease activity indices, physician and patient global assessment of disease activity and pain could be used for measuring disease exacerbation and flares in RA. Previous studies have reported pain as most important treatment priority for patients ${ }^{(45)}$. In other studies the physical function was found to be one of the best performing indicators of treatment benefits ${ }^{(46-51)}$. So, the poor performance of physical function in our analyses contrasts with previous studies.

In our analyses the CDAI, SDAI and DAS28 performed equivalently as measures of disease exacerbation. Similar results were observed for these three scores in two previous studies (52,53). Acute-phase reactants in our study seems to give a little additional information on disease activity exacerbation. 
We suggested that fatigue, emotional wellbeing or participation to a composite score may not contribute much to its reliability to predict flare. Previous studies also found limited responsiveness for participation and emotional wellbeing, But the value of fatigue reported different ${ }^{(54-59)}$.

\section{CONCLUSION}

Because of the potentially risks but especially because of increasing costs of longterm TNFi treatment, it was considered important to investigate whether established RA patients with sustained well controlled disease activity, defined as DAS28 remission or low disease activity can safely and effectively stop their therapy with TNFi. The primary study result is that within 12 months after stopping TNFi compared to those who continued TNFi experience a threefold increased risk of signs and symptoms of increased disease activity (flare). Of course stopping antirheumatic therapy in this case TNFi can only be considered in patients in whom the treatment goal is reached. It does not need to be advocated that In case treatment goals are not reached treatment escalation is indicated. We do realize that an intense discussion on the definition of remission is ongoing. Our result support the need of a definition of remission without residual disease activity, although operationalization in a global applicable definition remains a challenge beyond the goals of this thesis. The definition of increased disease activity (flare) differs between studies, we did do sensitivity analysis using different definition of flare all coming to the same conclusion.

Taking into account that RA is a live long disease discontinuation of successful antirheumatic therapy remains a risk. Successful stopping of TNFi seems to be possible for a subgroup of RA patients but comes with an increased risk of flare that in the POET study and other studies was observed to be manageable. It is important to find out which group of patients have more risk of flare after discontinuation. We showed that discontinuation of TNFi does not have long-term consequences for the patients-related outcomes. Finely, Our analysis shows that stopping TNFis in RA patients with stable controlled disease is cost effective but results in a small QALY loss.

In conclusion, discontinuation of TNFi in well controlled RA patients is a realistic option Obviously, the decision to try to stop or taper TNFi must be a shared decision between rheumatologist and the patient. 


\section{REFERENCES}

1. Keane J, Gershon S, Wise RP, MirabileLevens E, Kasznica J, Schwieterman WD, Siegel JN, Braun MM. Tuberculosis associated with infliximab, a tumor necrosis factor alpha-neutralizing agent. $N$ Engl J Med. 2001 Oct 11;345(15):1098-104.

2. Carmona L, Gómez-Reino JJ, RodríguezValverde V, Montero D, Pascual-Gómez E, Mola EM, Carreño L, Figueroa M; BIOBADASER Group. Effectiveness of recommendations to prevent reactivation of latent tuberculosis infection in patients treated with tumor necrosis factor antagonists. Arthritis Rheum. 2005 Jun;52(6):1766-72.

3. Goh L(1), Jewell T, Laversuch C, Samanta A. Should anti-TNF therapy be discontinued in rheumatoid arthritis patientsundergoing elective orthopaedic surgery? A systematic review of the evidence. Rheumatol Int. 2012 Jan;32(1):5-13. doi: 10.1007/s00296011-2040-6. Epub 2011 Jul 29.

4. Schipper LG, Vermeer M, Kuper $\mathrm{HH}$, Hoekstra MO, Haagsma CJ, Den Broeder AA, van Riel P, Fransen J, van de Laar MA. A tight control treatment strategy aiming for remission in early rheumatoid arthritis is more effective than usual care treatment in daily clinical practice: a study of two cohorts in the Dutch Rheumatoid Arthritis Monitoring registry. Ann Rheum Dis. 2012 Jun;71(6):84550. doi: 10.1136/annrheumdis-2011-200274. Epub 2011 Dec 30

5. Smolen JS, Aletaha D, Bijlsma JW, Breedveld FC, Boumpas D, Burmester G, Combe B, Cutolo M, de Wit M, Dougados
M, Emery P, Gibofsky A, Gomez-Reino JJ, Haraoui B, Kalden J, Keystone EC, Kvien TK, Mclnnes I, Martin-Mola E, Montecucco C, Schoels M, van der Heijde D; T2T Expert Committee. Treating rheumatoid arthritis to target: recommendations of an international task force. Ann Rheum Dis. 2010 Apr;69(4):631-7. doi: 10.1136/ ard.2009.123919. Epub 2010 Mar 9. Erratum in: Ann Rheum Dis. @011 Jul;70(7):1349. van der Heijde, Desirée [corrected to van der Heijde, Désirée]. Ann Rheum Dis. 2011 Aug;70(8):1519.

6. Boehncke $\mathrm{WH}$, Brembilla NC. Immunogenicity of biologic therapies: causes and consequences. Expert Rev Clin Immunol. 2018 Jun;14(6):513-523. doi: 10.1080/1744666X.2018.1468753.

7. Kavanaugh A, Lee SJ, Curtis JR, Greenberg JD, Kremer JM, Soto L, et al. Discontinuation of tumour necrosis inhibitors in factor patients with rheumatoid arthritis in lowdisease activity: persistent benefits. Data from the Corrona registry. Ann Rheum Dis. 2014 Dec 3;0:1-6.

8. Nam JL, Villeneuve E, Hensor EMA, Wakefield RJ, Conaghan PG, Green MJ, et al. A randomised controlled trial of etanercept and methotrexate to induce remission in early inflammatory arthritis: the EMPIRE trial. Ann Rheum Dis. 2014;73(6):1027-36.

9. Smolen JS, Nash P, Durez P, et al. Maintenance, reduction, or withdrawal of etanercept after treatment with etanercept and methotrexate in patients with moderate rheumatoid arthritis (PRESERVE): 
a randomised controlled trial. Lancet. 2013;381:918-29.

10. Van Vollenhoven RF, Ostergaard $M$, Leirisalo-Repo M, Uhlig $T$, Jansson $M$, Larsson E, et al. Full dose, reduced dose or discontinuation of etanercept in rheumatoid arthritis. Ann Rheum Dis. 2016 Jan;75(1):528. doi: 10.1136/annrheumdis-2014-205726. Epub 2015 Apr 14.

11. Smolen JS, Emery P, Fleischmann R, et al. Adjustment of therapy in rheumatoid arthritis on the basis of achievement of stable low disease activity with adalimumab plus methotrexate or methotrexate alone: the randomised controlled OPTIMA trial. Lancet. 2014;383:321-32.

12. Hirata S, Saito K, Kubo S, Fukuyo S, Mizuno Y, Iwata S, et al. Discontinuation of adalimumab after attaining disease activity score 28-erythrocyte sedimentation rate remission in patients with rheumatoid arthritis (HONOR study): an observational study. Arthritis Res Ther. 2013 Jan;15(5):R135.

13. Harigai M, Takeuchi T, Tanaka Y, Matsubara T, Yamanaka H, Miyasaka N. Discontinuation of adalimumab treatment in rheumatoid arthritis patients after achieving low disease activity. Mod Rheumatol. 2012;22(6):81422.

14. QuinnMA, Conaghan PG, O'Connor PJ, et al. Very early treatment with infliximab in addition to methotrexate in early, poorprognosis rheumatoid arthritis reduces magnetic resonance imaging evidence of synovitis and damage, with sustained benefit after infliximab withdrawal: results from a twelve-month randomized, doubleblind, placebo-controlled trial. Arthritis Rheum 2005;52:27-35.

15. van den Broek M, Klarenbeek NB, Dirven L, van Schaardenburg D, Hulsmans HMJ, Kerstens PJSM, et al. Discontinuation of infliximab and potential predictors of persistent low disease activity in patients with early rheumatoid arthritis and disease activity score-steered therapy: subanalysis of the BeSt study.Ann Rheum Dis. 2011;70(8):1389-94.

16. Tanaka $\mathrm{Y}$, Takeuchi T, Mimori T, Saito K, Nawata M, Kameda H, et al. Discontinuation of infliximab after attaining low disease activity in patients with rheumatoid arthritis: RRR (remission induction by Remicade in RA) study. Ann Rheum Dis. 2010;69(7):1286-91.

17. Smolen JS, Emery P, Ferraccioli GF, Samborski W, Berenbaum F, Davies OR, et al. Certolizumab pegol in rheumatoid arthritis patients with low to moderate activity: the CERTAIN double-blind, randomised, placebo-controlled trial. Ann Rheum Dis. 2015;74(5):843-50.

18. Van Herwaarden N, van der Maas A, Minten MJM, van den Hoogen FHJ, Kievit W, van Vollenhoven RF, et al. Disease activity guided dose reduction and withdrawal of adalimumab or etanercept compared with usual care in rheumatoid arthritis: open label, randomised controlled, noninferiority trial. BMJ. 2015 Jan;350:h1389.

19. Fautrel B, Pham T, Alfaiate T, et al. Step-down strategy of spacing TNF-blocker injections 
for established rheumatoid arthritis in remission: results of the multicentre non-inferiority randomised open-label controlled trial (STRASS: spacing of TNFblocker injections in Rheumatoid ArthritiS Study). Ann Rheum Dis. 2016;75:59-67.

20. Haschka J, Englbrecht M, Hueber AJ, Manger B, Kleyer A, Reiser $M$, et al. Relapse rates in patients with rheumatoid arthritis in stable remission tapering or stopping antirheumatic therapy: interim results from the prospective randomised controlled RETRO study. Ann Rheum Dis. 2016;75(1):45-51.

21. Smolen JS, Nash P, Durez $P$, Hall $S$, Ilivanova E, Irazoque-Palazuelos $F$, et al. Maintenance, reduction, or withdrawal of etanercept after treatment with etanercept and methotrexate in patients with moderate rheumatoid arthritis (PRESERVE): a randomised controlled trial. Lancet. 2013 Mar 16;381(9870):918-29.

22. Van Vollenhoven RF, Ostergaard $M$, Leirisalo-Repo $M$, Uhlig $T$, Jansson $M$, Larsson E, et al. Full dose, reduced dose or discontinuation of etanercept in rheumatoid arthritis. Ann Rheum Dis. 2015 Apr 14;

23. Botsios C, Furlan A, Ostuni R, Sfriso R, Todesco S, Punzi L. Effects of low-dose etanercept in maintaining DAS-remission previously achieved with standard-dose in patients with rheumatoid arthritis. Ann Rheum Dis. 2007 Jul;66:54-54.

24. Emery $P$, Hammoudeh $M$, FitzGerald $O$, Combe B, Martin Mola E, Bukowski J, et al. Assessing maintenance of remission with reduced dose etanercept plus methotrexate, methotrexate alone, or placebo in patients with early rheumatoid arthritis who achieved remission with etanercept and methotrextate: the prize study. Ann Rheum Dis. 2014 Jan 23;72(Suppl 3):A399-A399.

25. Kavanaugh A, Smolen JS. The when and how of biologic agent withdrawal in rheumatoid arthritis: learning from large randomised controlled trials. Clin Exp Rheumatol. 2013;31(4 Suppl 78):S19-21.

26. Kuijper TM, Lamers-Karnebeek FB, Jacobs JW, Hazes JM, Luime JJ. Flare rate in patients with rheumatoid arthritis in low disease activity or remission when tapering or stopping synthetic or biologic DMARD: a systematic review. J Rheumatol. 2015;42(11):2012-22.

27. Saleem B, Keen $H$, Goeb V, Parmar R, Nizam S, Hensor EMA, et al. Patients with $\mathrm{RA}$ in remission on TNF blockers: when and in whom can TNF blocker therapy be stopped? Ann Rheum Dis. 2010;69(9):163642.

28. Hashimoto M, Furu M, Yamamoto W, Fujimura T, Hara R, Katayama M, Ohnishi A, Akashi K, Yoshida S, Nagai K, Son Y, Amuro $\mathrm{H}$, Hirano $\mathrm{T}$, Ebina $\mathrm{K}$, Uozumi R, Ito H, Tanaka M, Ohmura K, Fujii T, Mimori T. Factors associated with the achievement of biological disease-modifying antirheumatic drug-free remission in rheumatoid arthritis: the ANSWER cohort study. Arthritis Res Ther. 2018 Aug 3;20(1):165. doi: 10.1186/s13075-018-1673-1. PubMed 
PMID: 30075810; PubMed Central PMCID: PMC6091083.

29. Centola M, Cavet G, Shen Y, Ramanujan S, Knowlton N, Swan KA, et al. Development of a multi-biomarker disease activity test for rheumatoid arthritis. PLoS One. 2013;8: e60635. doi:10.1371/journal.pone.0060635.

30. Curtis JR, van der Helm-van Mil AH, Knevel R, Huizinga TW, Haney DJ, Shen Y, et al. Validation of a novel multibiomarker test to assess rheumatoid arthritis disease activity. Arthritis Care Res (Hoboken). 2012;64: 1794-803. doi:10.1002/acr.21767.

31. Eastman PS, Manning WC, Qureshi F, Haney D, Cavet G, Alexander C, et al. Characterization of a multiplex, 12-biomarker test for rheumatoid arthritis. J Pharm Biomed Anal. 2012;70: 415-24. doi:10.1016/j.jpba.2012.06.003.

32. Hirata S, Li W, Defranoux N, Cavet G, Bolce $\mathrm{R}$, Yamaoka $\mathrm{K}$, et al. A multi-biomarker disease activity score tracks clinical response consistently in patients with rheumatoid arthritis treated with different anti-tumor necrosis factor therapies: A retrospective observational study. Mod Rheumatol. 2015;25: 344-9. doi:10.3109/14 397595.2014.958893.

33. van der Helm-van Mil AHM, Knevel R, Cavet G, Huizinga TWJ, Haney DJ. An evaluation of molecular and clinical remission in rheumatoid arthritis by assessing radiographic progression. Rheumatology (Oxford). 2013;52: 839-46. doi:10.1093/ rheumatology/kes378.

34. Hambardzumyan K, Bolce R, Saevarsdottir
S, Cruickshank SE, Sasso EH, Chernoff D, et al. Pretreatment multi-biomarker disease activity score and radiographic progression in early RA: results from the SWEFOT trial. Ann Rheum Dis. 2015;74: 1102-1109. doi:10.1136/annrheumdis-2013-204986.

35. Li W, Sasso EH, van der Helm-van Mil AHM, Huizinga TWJ. Relationship of multibiomarker disease activity score and other risk factors with radiographic progression in an observational study of patients with rheumatoid arthritis. Rheumatology (Oxford). 2016;55: 357-66. doi:10.1093/ rheumatology/kev341.

36. Rech J, Hueber AJ, Finzel S, Englbrecht M, Haschka J, Manger B, et al. Prediction of disease relapses by multibiomarker disease activity and autoantibody status in patients with rheumatoid arthritis on tapering DMARD treatment. Ann Rheum Dis. 2015;75: 1637-44. doi:10.1136/ annrheumdis-2015-207900.

37. Oderda GM, Lawless GD, Wright GC, Nussbaum SR, Elder R, Kim K, Brixner DI. The potential impact of monitoring disease activity biomarkers on rheumatoid arthritis outcomes and costs. Per Med. 2018 Jul 1;15(4):291-301. doi: 10.2217/pme-20180001. Epub 2018 Apr 25. PubMed PMID: 29693487.

38. Chatzidionysiou K, Turesson C, Teleman A, et al. A multicentre, randomised, controlled, open-label pilot study on the feasibility of discontinuation of adalimumab in established patients with rheumatoid arthritis in stable clinical remission. RMD 
Open. 2016;2(1):e000133.

39. Brocq O, Millasseau E, Albert C, et al. Effect of discontinuing TNFalpha antagonist therapy in patients with remission of rheumatoid arthritis. Jt Bone Spine. 2009;76(4):350-5.

40. Kievit W, van Herwaarden N, van den Hoogen FH, van Vollenhoven RF, Bijlsma JW, van den Bemt BJ, et al. Disease activity-guided dose optimisation of adalimumab and etanercept is a costeffective strategy compared with nontapering tight control rheumatoid arthritis care: analyses of the DRESS study.Ann Rheum Dis. 2016;75(11):1939-44.

41. Vanier A, Tubach F, Alfaiate T, Mariette X, Fautrel B. Step-down strategy of spacing TNF-blockers injections for established rheumatoid arthritis in remission: a costutility analysis based on the STRASS trial. Ann Rheum Dis. 2015;74(Suppl 2):178.

42. Kobelt $\mathrm{G}$. Treating to target with etanercept in rheumatoid arthritis: cost-effectiveness of dose reductions when remission is achieved. Value Health. 2014;17(5):537-44.

43. van der Maas A, Kievit W, van den Bemt BJ, van den Hoogen $\mathrm{FH}$, van Riel PL, den Broeder AA. Down-titration and discontinuation of infliximab in rheumatoid arthritis patients with stable low disease activity and stable treatment: an observational cohort study.

44. Murphy CL, Awan S, Sullivan MO, et al. Major cost savings associated with biologic dose reduction in patients with inflammatory arthritis. Ir Med J.2015;108(1):19-21.
45. Klooster PM ten, Veehof MM, Taal E, Riel PLCM van, Laar MAFJ van de. Changes in priorities for improvement in patients with rheumatoid arthritis during 1 year of anti-tumour necrosis factor treatment. Ann Rheum Dis 2007;66:1485-90.

46. Buchbinder R, Bombardier C, Yeung M, Tugwell P. Which outcome measures should be used in rheumatoid arthritis clinical trials? Clinical and quality-of-life measures' responsiveness to treatment in a randomized controlled trial. Arthritis Rheum 1995;38:1568-80.

47. Anderson JJ, Chernoff MC. Sensitivity to change of rheumatoid arthritis clinical trial outcome measures. J Rheumatol 1993;20:535-7.

48. Verhoeven AC, Boers M, Linden S van Der. Responsiveness of the core set, response criteria, and utilities in early rheumatoid arthritis. Ann Rheum Dis 2000;59:966-74.

49. Russell AS, Conner-Spady B, Mintz A, Maksymowych WP. The responsiveness of generic health status measures as assessed in patients with rheumatoid arthritis receiving infliximab. J Rheumatol 2003;30:941-7.

50. Tugwell P, Wells G, Strand V, Maetzel A, Bombardier C, Crawford B, et al. Clinical improvement as reflected in measures of function and health-related quality of life following treatment with leflunomide compared with methotrexate in patients with rheumatoid arthritis: sensitivity and relative efficiency to detect a treatment e. Arthritis Rheum 2000;43:506-14. 
51. Cohen SB, Strand V, Aguilar D, Ofman JJ. Patient- versus physician-reported outcomes in rheumatoid arthritis patients treated with recombinant interleukin-1 receptor antagonist (anakinra) therapy. Rheumatology (Oxford) 2004;43:704-11.

52. Aletaha D, Nell VPK, Stamm T, Uffmann M, Pflugbeil S, Machold K, et al. Acute phase reactants add little to composite disease activity indices for rheumatoid arthritis: validation of a clinical activity score. Arthritis Res Ther 2005;7:R796-806.

53. Ranganath VK, Yoon J, Khanna D, Park GS, Furst DE, Elashoff DA, et al. Comparison of composite measures of disease activity in an early seropositive rheumatoid arthritis cohort. Ann Rheum Dis 2007;66:1633-40.

54. Aletaha D, Landewe R, Karonitsch T, Bathon J, Boers M, Bombardier C, et al. Reporting disease activity in clinical trials of patients with rheumatoid arthritis: EULAR/ ACR collaborative recommendations. Ann Rheum Dis 2008;67:1360-4.

55. Buchbinder R, Bombardier C, Yeung M, Tugwell P. Which outcome measures should be used in rheumatoid arthritis clinical trials? Clinical and quality-of-life measures' responsiveness to treatment in a randomized controlled trial. Arthritis Rheum 1995;38:1568-80

56. Anderson JJ, Chernoff MC. Sensitivity to change of rheumatoid arthritis clinical trial outcome measures. J Rheumatol 1993;20:535-7.

57. Verhoeven AC, Boers M, Linden S van Der. Responsiveness of the core set, response criteria, and utilities in early rheumatoid arthritis. Ann Rheum Dis 2000;59:966-74.

58. Russell AS, Conner-Spady B, Mintz A, Maksymowych WP. The responsiveness of generic health status measures as assessed in patients with rheumatoid arthritis receiving infliximab. J Rheumatol 2003;30:941-7.

59. Tugwell P, Wells G, Strand V, Maetzel A, Bombardier C, Crawford B, et al. Clinical improvement as reflected in measures of function and health-related quality of life following treatment with leflunomide compared with methotrexate in patients with rheumatoid arthritis: sensitivity and relative efficiency to detect a treatment $\mathrm{e}$. Arthritis Rheum 2000;43:506-14. 
GENERAL DISCUSSION 



\section{$\mathbf{A}$}

Nederlandse samenvatting

Acknowledgements/Dankwoord

About the Author

List of Publications 
ADDENDUM 


\section{SAMENVATTING EN ALGEMENE BESCHOUWING}

Rond de eeuwwisseling begon de behandeling van Reumatoïde Artritis (RA) te veranderen van zorgen voor de patiënten naar een situatie waarin genezing een reële optie is geworden voor patiënten. De basis voor deze verandering was de ontwikkeling en introductie van remmers van Tumor Necrosis Factor (TNFi) en later andere therapeutische aangrijpingspunten. Achteraf gezien blijkt de effectiviteit van deze nieuwe klasse van geneesmiddelen, in het algemeen biologicals genoemd, beter dan verwacht, waardoor de prognose en het perspectief van veel RA patiënten en patiënten die lijden aan andere auto-immuunziekten verbeterde. Tijdens de ontwikkeling en introductie van TNFi was de aandacht, begrijpelijk, vooral gefocust op effectiviteit en veiligheid na start van de behandeling. Verlaging van de dosis, of stoppen van de behandeling, in geval van gunstige behandelresultaten kreeg nauwelijks aandacht of werd niet overwogen. De veronderstelling dat auto-immuunziekten zoals RA chronische ziekten zijn, die levenslang behandeld dienen te worden, en in het beste geval slechts progressie kan worden afgeremd, bleef de leidende gedachte van zowel professionals als patiënten.

We werden voor het eerst geconfronteerd met de noodzaak om behandeling met TNFi te stoppen of te onderbreken toen het, weliswaar verwachte, toegenomen risico op infectie realiteit bleek te zijn (1). Richtlijnen om het infectierisico als gevolg van remming van TNF te verminderen werden ontwikkeld en succesvol geïmplementeerd ${ }^{(2)}$. Hoewel nauwelijks onderzocht, bestond de indruk bij behandelaars dat na stoppen van TNFi in verband met infectie of verhoogd infectierisico (met name geplande operaties), toename van ziekteactiviteit voorkomt, maar dat hervatten van de behandeling leidde tot snelle herwonnen respons ${ }^{(3)}$.

Studies naar het induceren van remissie ${ }^{(4)}$, later meestal "treating to the target of remission" (T2T) genoemd ${ }^{(5)}$, was een direct gevolg van de indrukwekkende klinische effecten van de introductie van biologicals. Het hoge percentage RA patiënten in studies in de dagelijkse praktijk bij wie stabiele remissie kon worden bereikt door gebruik te maken van de therapeutische mogelijkheden, in combinatie met de toegenomen geneesmiddelkosten en zorgen over levenslange behandeling, bereidde de weg voor studies naar dosisverlaging of stoppen van TNFi in geval van goede klinische effecten (remissie of lage ziekte activiteit). Hoewel wetenschappelijke argumenten tegen dosisverlaging, met name immunogeniciteit ${ }^{(6)}$, sterker zijn dan de argumenten tegen meteen stoppen, zijn met name psychologische argumenten van patiënten en behandelaars de oorzaak dat de meerderheid van de gedane studies de focus hebben gelegd op dosisverlaging. De meeste studies werden gestart op initiatief van de onderzoeker en daarom begrijpelijk van 
geringe omvang en onderhevig aan methodologische beperkingen. De POEET studie, de basis voor dit proefschrift, was een gecombineerd initiatief van Nederlandse reumatologen en zorgbeleidmakers. Deze gezamenlijke Nederlandse inspanning heeft geresulteerd in een van de grootste studies naar doelmatig gebruik van biologicals in RA, in dit geval door te stoppen. Hoewel ook deze studie beperkingen kent, zoals het open-label en pragmatische studieontwerp, maakt het indrukwekkende aantal deelnemende patiënten het mogelijk niet alleen de hoofdvraag van de studie maar ook extra onderzoeksvragen, zoals de betrokken mechanismen en de invloed op patiënten en de maatschappij, te onderzoeken.

In het eerste hoofdstuk van dit proefschrift heb ik bestaande informatie over stoppen en afbouwen van biologicals voor RA besproken in combinatie met een theoretisch schema voor de mogelijk onderliggende mechanismes.

\section{HOOFDSTUK 2}

Dit hoofdstuk is de eerste en meeste belangrijke publicatie van de POEET studie. Het was mijn eer om, namens het POEET team, te laten zien dat stoppen van TNFi mogelijk was bij $48 \%$ van RA patiënten in stabiele remissie of lage ziekteactiviteit (LDA). Maar de stopstrategie resulteerde wel in een meer dan drievoudig toegenomen kans op klachten en verschijnselen van toegenomen ziekteactiviteit (exacerbatie) binnen 12 maanden ten opzichte van RA patiënten die hun TNFi bleven gebruiken. We hebben ook laten zien dat de gemiddelde ziekteactiviteit in de stopgroep significant verhoogd was gedurende de observatieperiode, maar in de meeste patiënten bleef de ziekteactiviteit onder de grens van matige activiteit. Omdat verschillende definities van exacerbatie in de literatuur gebruikt worden, hebben we sensitiviteitsanalyses uitgevoerd met verschillende definities, hetgeen vergelijkbare resultaten aantoonde. De resultaten van onze studie bevestigen in grote lijnen de bevindingen van eerdere, veelal kleinere studies.

Eerdere TNFi stop studies in RA lieten een brede spreiding (18-88\%) zien van het risico op exacerbatie van ziekteactiviteit. De verschillen in deze resultaten kunnen onder andere verklaard worden door de heterogeniteit van de RA populaties, verschillende definities van exacerbatie, (rest) ziekteactiviteit bij inclusie en het parallel gebruik van andere DMARDs. Gerandomiseerde studies naar het stoppen van specifieke TNFi's rapporteerden ook verschillende resultaten. De Amerikaanse "Corrona registry" studie rapporteerde dat ruim $70 \%$ van de RA patiënten gedurende 12 maanden observatie na het stoppen van een eerste TNFI geen exacerbatie meemaakten (7). De EMPIRE trial, PRESERVE trial, 
en DOSERA studies meldden dat respectievelijk ongeveer 57\%, 43\% en 13\% van de RA patiënten succesvol konden stoppen met etanercept tijdens de studie ${ }^{(8-10)}$. De OPTIMA trial, HONOR en BRIGHT studies rapporteerden dat respectievelijk 56\%, 58\% en $18 \%$ van de RA patiënten in remissie of lage ziekteactiviteit bleven na stoppen van hun TNFi ${ }^{(11-13)}$. Het stoppen van infliximab werd beschreven in de studie van Quinn et al., de BeSt studie en de "Remission induction by Remicade in RA" (RRR-studie). De studies rapporteerden blijvende lage ziekteactiviteit of remissie in respectievelijk $70 \%, 25 \%$ en 50\% van de patiënten. Als laatste rapporteerde een kleine studie over stoppen van certolizumabpegol, in tegenstelling tot de hiervoor genoemde studies, geen positief resultaat ${ }^{(17)}$.

In onze POEET studie werd, in geval van exacerbatie na stoppen van TNFi, geadviseerd de TNFi te hervatten. De geobserveerde data laten zien dat herbehandeling resulteerde in succes bij de meeste patiënten (83.1\% succes na hervatten TNFi) in de meeste gevallen binnen 12 weken. Aanvullend werden er geen (ernstige) negatieve gebeurtenissen geobserveerd na hervatten van TNFi. Tenslotte hebben we geobserveerd dat in de stopgroep ten opzichte van de continueergroep meer ziekenhuisopnames plaatsvonden, weliswaar volgens de behandelaar niet gerelateerd aan het stoppen. De redenen voor opname in een ziekenhuis waren: infecties, geplande operaties, maligniteiten en fracturen. We zijn tot de conclusie gekomen dat het stoppen van TNFI bij RA patiënten en hervatten van TNFI in geval van exacerbatie veilig lijkt te zijn.

In aanvulling op het stoppen van TNFi zijn er meerdere strategieën denkbaar voor verlaging van de dosis. Vanzelfsprekend overschrijdt de variabiliteit hiervoor de opties voor onmiddellijk stoppen, zoals werd gedaan in de POEET studie. De PRESERVA, DOSERA en DRESS studies gebruikten een verschillende afbouwstrategie ten opzichte van de STRASS en de RETRO studie ${ }^{(9,10,18-20)}$. In het algemeen lieten deze studies zien dat minder exacerbaties optraden tijdens dosisverlagingen in vergelijking met stoppen, maar op langere termijn zijn de resultaten van dosisreductieschema's en meteen stoppen vergelijkbaar. Het risico op exacerbatie tijdens dosisreductie is lager dan dat tijdens meteen stoppen, maar het cumulatieve risico van alle sequentiële dosisverlagingen is goed vergelijkbaar met het risico op exacerbaties na volledig stoppen ${ }^{(9,10,21,22)}$. Recent liet de DRESS studie zien dat ziekteactiviteit-gestuurd dosis verlagen van adalimumab en etanercept "non-inferior" is met betrekking tot ernstige exacerbaties ten opzichte van doorgaan van de medicatie ${ }^{(18)}$. 


\section{HOOFDSTUK 3}

In dit hoofdstuk gingen we op zoek naar voorspellers voor het succesvol (niet resulterend in een exacerbatie van ziekteactiviteit) stoppen van TNFi. Dit hoofdstuk helpt de theorie zoals beschreven in hoofdstuk 1 te begrijpen en verder te vormen. Onze analyse liet zien dat $70 \%$ van de RA patiënten in stabiele remissie of lage ziekteactiviteit die gekenmerkt werden door het gebruik van een TNFi van de antilichaam subklasse, met een relatief korte ziekte duur van minder dan 10 jaar en een lage MBDA score van kleiner of gelijk aan 44, in staat waren hun TNFi te stoppen zonder dat een exacerbatie volgde gedurende het volgende jaar. Daarentegen moest $80 \%$ van vergelijkbare RA patiënten die die een TNFi van de receptor antagonist klasse gebruikte, met een ziekte duur van 10 jaar of langer en een MBDA score van boven de 44 hun TNFi hervatten vanwege klachten of verschijnselen wijzend op exacerbatie van hun RA.

De observatie van de POEET studie dat relatief korte ziekteduur gerelateerd is aan het risico op exacerbaties is in overeenstemming met de studie van Kavanaugh et al., die meldde dat succesvol stoppen van bDMARDS mogelijk was bij RA patiënten met korte ziekteduur ${ }^{(23)}$. Kuiper et al., daarentegen, waren niet in staat te laten zien dat de ziekteduur van RA patiënten gerelateerd was aan het al dan niet kunnen stoppen van de TNFi behandeling ${ }^{(24)}$. Mogelijk is het negatieve resultaat van deze laatste studie te verklaren door het beperkte aantal patiënten.

In onze studie waren aanwezigheid van reumafactoren en BMI niet gerelateerd aan het effect van stoppen van TNFi. In tegenstelling tot de Corrona registry en RRR studies die wel een relatie vonden tussen deze predictoren en succesvol stoppen van $\mathrm{TNFi}^{(7,16)}$. Een mogelijke verklaring voor de afwezigheid van een relatie met reumafactor en anti-CCP zou het hoge percentage dubbel positieve patiënten in onze studie kunnen zijn. Klachten of verschijnselen van resterende ziekteactiviteit ten tijde van het stoppen lijken wel een predictor te zijn van exacerbatie na het stoppen van TNFi ${ }^{(13,16,25)}$. De associatie met een hoge MBDA score bij studiestart in onze POEET studie lijkt in overeenstemming met deze hypothese.

We vonden daarnaast dat het type TNFi ook een predictor lijkt te zijn. Onze analyses laten zien dat patiënten die een TNFi uit de antilichaam klasse (voornamelijk adalimumab) gebruikten significant vaker succesvol hun TNFi konden stoppen in vergelijking met patiënten die een TNFi receptor anatagonist (voornamelijk etanercept) gebruikten. TNFi antilichamen lijken een langdurige ziektecontrole te induceren, mogelijk gerelateerd aan de lange halfwaardetijd, door inductie van apoptose van TNF bindende of dragende bloedcellen. Onze bevindingen komen overeen met de recente studie van Hashimoto et al. ${ }^{(26)}$. 
De informatie verkregen in hoofdstuk 3, gecombineerd met de theorie uit hoofdstuk 1, lijkt steun te geven aan de "window of opportunity", de rol van resterende smeulende ziekteactiviteit, en het aangrijpingspunt / mechanisme van de TNFi bij het stoppen van TNFi. Vanzelfsprekend kunnen deze analyses, die achteraf zijn verricht, niet de theorie bewijzen maar wel steunen ze de onderliggende hypothesen.

\section{HOOFDSTUK 4}

De multi-biomarker disease activity (MBDA) bloedtest maakt gebruik van 12 biomarkers om een score te berekenen die valide is gebleken als ziekteactiviteitparameter voor RA patiënten ${ }^{(27-29)}$. MBDA scores reflecteren de actuele ziekteactiviteit en veranderingen daarvan door de tijd, inclusief effecten van behandelingen met TNFi van RA patiënten (30). In dit vierde hoofdstuk bestudeerden we deze MBDA score als een voorspeller van exacerbatie na stoppen van TNFi behandeling. We gebruikten de gegevens van de patiënten die gerandomiseerd waren naar de stopgroep. Van nagenoeg al deze patiënten waren serum monsters ten tijde van de start van de studie aanwezig om de MBDA test te doen waardoor de baseline score gebruikt konden worden. We lieten zien dat patiënten met een relatief hoge MBDA score ten tijde van het stoppen van TNFi, significant vaker een exacerbatie kregen tijdens de twaalf maanden observatie. De resultaten suggereren dat een hoge MBDA score, hoewel de patiënten in remissie of lage ziekteactiviteit zijn, een teken kan zijn van resterende ziekteactiviteit. Verschillende voorgaande studies hebben verhoogde MBDA scores in RA patiënten in remissie of lage ziekteactiviteit gevonden (31-34). We konden, naar ons weten, als eerste melden dat de MBDA score voorspellende waarde heeft voor het risico op exacerbatie na stoppen van TNFi.

Ten aanzien van de drie verschillende definities van exacerbatie in de POEET studie (TNFi hervatten, medicatie-escalatie en exacerbatie gerapporteerd door de behandelaar), was een hoge MBDA score voor elke definitie steeds een onafhankelijke voorspeller van exacerbatie, maar dit bleef niet zo als we corrigeerden voor alle potentiele voorspellers inclusief de DAS28 scores.

Hoewel de heterogeniteit van RA, en als consequentie daarvan het moeilijk vaststellen van ziekteactiviteit, goed bekend is, lijken laboratoriummethodes (biomarkers) zoals de MBDA score niet in staat de beperkingen van samengestelde klinische uitkomstmaten te verbeteren. De resultaten van de POEET studie bevestigen dat er een relatie bestaat tussen RA ziekteactiviteit en de MBDA score, maar deze relatie is niet erg sterk en rechtvaardigt mogelijk niet de kosten van de test ${ }^{(35)}$. 


\section{HOOFDSTUK 5}

Naast het biomedische perspectief van de ziekte, is conceptueel het perspectief van de patiënt de spiegel van de ziekteconsequenties voor de patiënt en diens gezondheidsstatus. Daarom hebben we in de POEET studie in aanvulling op de biomedische resultaten ook onderzoek gedaan naar de gevolgen voor de patiënt. We hebben aangetoond dat stoppen van TNFi een significant maar kortdurend effect heeft op patiënt-gerapporteerde uitkomstmaten (PROs), zoals de fysieke en geestelijke gezondheidsstatus en gezondheidsgerelateerde utiliteit, pijn, beperkingen en moeheid.

Slechts enkele andere studies analyseerden het effect van TNFi stoppen op de door de patiënten ervaren ziektelast. De gerandomiseerde PRESERVE trial liet na 12 maanden zien dat patiënten die etanercept met methotrexaat bleven gebruiken beter scoorden ten aanzien van algemene gezondheid, pijn, beperkingen, gezondheidsutiliteiten, slaap en moeheid in vergelijking met de patiënten die etanercept stopten en alleen methotrexaat gebruikten ${ }^{(9)}$. Aanvullend liet de observationele RRR studie na 1 jaar zien dat de gemiddelde fysieke beperkingen (HAQ-DI scores) lager (beter) waren in patiënten die in remissie bleven na het stoppen van infliximab in vergelijking tot patiënten die een ziekte-exacerbatie doormaakten na stoppen ${ }^{(16)}$. Dit resultaat werd echter niet gevonden in de OPTIMA of de ADMIRE studie ${ }^{(11,36)}$. Ook de resultaten van de open-label, niet gerandomiseerde HONOR studie liet geen verschil zien ten aanzien van HAQ-DI scores een jaar na stoppen van adalimumab tussen patiënten die in remissie bleven of patiënten die een exacerbatie kregen ${ }^{(12)}$. In een erg kleine observationele studie van 21 patiënten, vonden Brocq et al. geen significante verschillen gedurende een jaar ten aanzien van HAQ-DI scores in patiënten die TNFi stopten ${ }^{(37)}$.

In het algemeen kunnen we concluderen dat de negatieve biomedische effecten van het stoppen van TNFi in RA patiënten ook te zien zijn in de ongunstigere patiënt-gerapporteerde uitkomsten. Maar, in overeenstemming met de zorgvuldige zorg ontwerpen van de verschillende studies, zijn de ongunstigere uitkomsten gering en van korte duur, zonder noemenswaardige invloed op de lange termijn gezondheid van patiënten die proberen hun kostbare behandelingen te stoppen of de dosis ervan te verminderen.

\section{HOOFDSTUK 6}

Omdat de kosten van geneesmiddelen een van de belangrijke argumenten was in de discussie over stoppen van biologicals, is een kostenanalyse van stoppen versus 
doorgaan met TNFi behandeling in het geval van goede ziektecontrole (remissie of LDA, zoals gedefinieerd door de DAS28) vanzelfsprekend relevant. We hebben laten zien dat stoppen met TNFi in deze groep RA patiënten kosten kan besparen, maar dit betekent wel dat er een klein verlies optreedt aan gezondheidsutiliteiten, zoals gemeten middels quality-adjusted life years (QALYs). De gemiddelde bespaarde kosten waren $€ 368$.269,per verloren QALY. We lieten zien dat, met uitzondering van het eerste evaluatiemoment na stoppen, de gemiddelde gezondheidsutiliteit niet significant verschilde gedurende de studieperiode tussen patiënten die stopten of doorgingen met hun TNFi. De resultaten suggereren dat stoppen van TNFI in goed gecontroleerde RA patiënten leidt tot aanzienlijke kostenbesparingen en beperkt en tijdelijk verlies aan gezondheidsgerelateerde kwaliteit van leven.

In de, ook Nederlandse, DRESS studie (dosis verlagen) lieten de onderzoekers zien dat gecontroleerde dosisverlaging van TNFi €390.493,- kan besparen voor elke verloren QALY (38), opvallend vergelijkbaar met stoppen van TNFi in onze POEET stop studie.

In een nadere kostenanalyse gebaseerd op de STRASS studie, rapporteerden de auteurs dat een dosisverlaging strategie $€ 53$.417,-- bespaart per verloren QALY (39). In de PRESERVE studie, eveneens met dosisreductie in geval van remissie als bestudeerde strategie, melden Kobelt et al. ${ }^{(40)}$ een bedrag tussen $€ 14.000$,- and $€ 29.000$,- per gewonnen QALY voor de dosis halvering strategie ten opzichte van de volledige stop van etanercept. De auteurs suggereerden dat een dosisreductie ten opzichte van stoppen de meest voordelige strategie is voor patiënten met milde RA, hoewel kostenbesparing van stoppen versus ongewijzigd doorgaan niet separaat gemeld werd ${ }^{(40)}$.

Sommige studie rapporteerden slechts de kosten van bespaarde geneesmiddelen. Van der Maas et al. rapporteerden een gemiddelde kostenbesparing van €3.474,- per patiënt ten gunste van dosisverlaging van infliximab ${ }^{(41)}$. Een vergelijkbare studie door Murphy et al. (42) in een cohort van 79 patiënten met behalve RA ook artritis psoriatica en ankyloserende spondylitis rapporteerde een gemiddelde besparing van €3.800,- per patient per jaar ten gunste van dosisreducties van etanercept of adalimumab ${ }^{(42)}$

\section{HOOFDSTUK 7}

In dit hoofdstuk verrichtten we een zogenaamde post-hoc analyse op de gegevens van de POEET studie om de meest bruikbare meetmethode vast te stellen voor exacerbatie van de ziekte. We toonden aan dat de samengestelde ziekteactiviteitsmaten, globale ziekteactiviteit inschattingen door behandelaar en door patiënt en zelfgerapporteerde 
pijnmaten allen gebruikt kunnen worden voor het meten van toename van ziekteactiviteit en opvlammingen van ziekte bij RA. Eerdere studies hebben gemeld dat pijn de belangrijkste prioriteit voor behandeling is voor patiënten ${ }^{(43)}$. In andere studies leek juist fysiek functioneren een van de beste indicatoren voor positieve behandelresultaten ${ }^{144-}$ ${ }^{49}$. Dus de slechte prestatie van fysiek functioneren in onze studie analyse contrasteert met voorgaande onderzoek naar verbeteringen in plaats van verslechteringen van RA ziekteactiviteit.

In onze analyse presteerden de CDAI, SDAI en DAS28 gelijkwaardig als proxy voor exacerbatie van ziekte activiteit. Vergelijkbare resultaten werden gemeld voor deze samengestelde scores in twee eerdere studies ${ }^{(50,51)}$. Acute-fase reacties daarentegen lijken weinig aanvullende waarde te hebben over exacerbatie van ziekteactiviteit. De resultaten bieden geen steun aan het recent gesuggereerde toevoegen van moeheid, emotioneel welbevinden of participatie aan een samengestelde maat voor ziekteactiviteit, omdat geen van deze maten veel bijdraagt aan de voorspelling van een exacerbatie van de ziekte. Eerdere studies konden ook geen relevante responsiviteit aantonen voor participatie en emotioneel welbevinden ${ }^{(44-48,52)}$.

\section{CONCLUSIE}

Vanwege de potentiele risico's maar vooral vanwege toenemende kosten van langdurige TNFi behandeling in groeiende patiëntaantallen, werd het belangrijk gevonden te onderzoeken of RA patiënten met goed gecontroleerde ziekteactiviteit, gedefinieerd als DAS28 remissie of lage ziekteactiviteit, veilig en succesvol hun behandeling met TNFI kunnen onderbreken. Het antwoord op de eerste studievraag is dat TNFi stoppen leidt tot een drievoudig verhoogd risico op exacerbatie van de ziekteactiviteit gedurende de studieduur van 12 maanden. Vanzelfsprekend kan het stoppen van anti-reumatische therapie, in dit geval TNFi behandeling, alleen worden overwogen in patiënten bij wie het beoogde behandelresultaat is behaald. Het behoeft geen betoogd dat in gevallen waar het behandelresultaat niet wordt gehaald, intensivering van de behandeling aanbevolen is. We realiseren ons dat een intensieve discussie over de operationalisatie van de definitie van remissie voortduurt. Onze studieresultaten ondersteunen de wenselijkheid van een definitie van remissie enerzijds zonder resterende, smeulende ziekteactiviteit als de patiënt voldoet aan de definitie, maar anderzijds zonder classificatie van patiënten zonder ziekteactiviteit als niet in remissie. Hoe dan ook, een wereldwijd bruikbare operationalisatie van de definitie van remissie blijft een uitdaging die verder reikt dan de scope van dit proefschrift. Hoewel de definitie van exacerbatie (flare) ook verschilt tussen 
studies, lieten wij zien dat een gevoeligheidsanalyse, gebruikmakende van meerdere definities van flare, geen invloed had op de conclusie.

Omdat RA een levenslange ziekte is, blijft het onderbreken van een succesvolle behandeling met anti-reumamedicatie een risico. Succesvol stoppen van TNFi lijkt echter een optie te zijn voor een subgroep van patiënten, maar betekent ook een risico op ziekte exacerbaties. Een risico dat in de POEET, en andere studies, gebleken is hanteerbaar te zijn. Het is belangrijk om van te voren zo goed mogelijk die patiënten te identificeren die het risico lopen op exacerbatie na stoppen versus hen die dat minder hebben. Wij lieten zien dat stoppen met TNFi geen langdurige consequenties lijkt te hebben voor uitkomsten die van belang zijn voor patiënten. Als laatste lieten onze bevindingen zien dat stoppen van TNFi in RA patiënten die goed onder controle zijn kosteneffectief is, maar resulteert in een klein verlies van gezondheidsutiliteit.

Concluderend, stoppen met TNFi in goed gecontroleerde RA patiënten is een realistische optie. Vanzelfsprekend dient het besluit te stoppen met TNFi, of om de dosis te verlagen, een gezamenlijke beslissing te zijn van de patiënt en de behandelend reumatoloog. 


\section{REFERENCES}

1. Keane J, Gershon S, Wise RP, MirabileLevens E, Kasznica J, Schwieterman WD, et al. Tuberculosis associated with infliximab, a tumor necrosis factor alpha-neutralizing agent. N Engl J Med. 2001;345(15):1098-104.

2. Carmona L, Gómez-Reino JJ, RodríguezValverde V, Montero D, Pascual-Gómez E, Mola EM, et al. Effectiveness of recommendations to prevent reactivation of latent tuberculosis infection in patients treated with tumor necrosis factor antagonists. Arthritis Rheum. 2005;52(6):1766-72.

3. Goh L, Jewell T, Laversuch C, Samanta A. Should anti-TNF therapy be discontinued in rheumatoid arthritis patients undergoing elective orthopaedic surgery? A systematic review of the evidence. Rheumatol Int. 2012;32(1):5-13.

4. Schipper LG, Vermeer $M$, Kuper $H H$, Hoekstra MO, Haagsma CJ, Broeder AA Den, et al. A tight control treatment strategy aiming for remission in early rheumatoid arthritis is more effective than usual care treatment in daily clinical practice: a study of two cohorts in the Dutch Rheumatoid Arthritis Monitoring Registry. Ann Rheum Dis. 2012;71(1):845-50.

5. Smolen JS, Aletaha D, Bijlsma JWJ, Breedveld FC, Boumpas D, Burmester G, et al. Treating rheumatoid arthritis to target: recommendations of an international task force. Ann Rheum Dis. 2010;69(4):631-7.

6. Boehncke $\mathrm{W}-\mathrm{H}$, Brembilla NC. Immunogenicity of biologic therapies: causes and consequences. Expert Rev Clin
Immunol. 2018;14(6):513-23.

7. Kavanaugh A, Lee SJ, Curtis JR, Greenberg JD, Kremer JM, Soto L, et al. Discontinuation of tumour necrosis factor inhibitors in patients with rheumatoid arthritis in lowdisease activity: persistent benefits. Data from the Corrona registry. Ann Rheum Dis. 2015;7(4):1150-5.

8. Nam JL, Villeneuve E, Hensor EMA, Wakefield RJ, Conaghan PG, Green MJ, et al. A randomised controlled trial of etanercept and methotrexate to induce remission in early inflammatory arthritis: the EMPIRE trial. Ann Rheum Dis. 2014;73(6):1027-36.

9. Smolen JS, Nash P, Durez P, Hall S, Ilivanova E, Irazoque-Palazuelos $F$, et al. Maintenance, reduction, or withdrawal of etanercept after treatment with etanercept and methotrexate in patients with moderate rheumatoid arthritis (PRESERVE): a randomised controlled trial. Lancet. 2013;381(9870):918-29.

10. van Vollenhoven RF, Ostergaard $M$, Leirisalo-Repo $M$, Uhlig $T$, Jansson $M$, Larsson E, et al. Full dose, reduced dose or discontinuation of etanercept in rheumatoid arthritis. Ann Rheum Dis. 2016;75(1):52-8.

11. Smolen JS, Emery P, Fleischmann R, van Vollenhoven RF, Pavelka K, Durez P, et al. Adjustment of therapy in rheumatoid arthritis on the basis of achievement of stable low disease activity with adalimumab plus methotrexate or methotrexate alone: the randomised controlled OPTIMA trial. Lancet. 2014;383(9914):321-32.

12. Hirata S, Saito K, Kubo S, Fukuyo S, 
Mizuno Y, Iwata S, et al. Discontinuation of adalimumab after attaining disease activity score 28-erythrocyte sedimentation rate remission in patients with rheumatoid arthritis (HONOR study): an observational study. Arthritis Res Ther. 2013 Sep 25;15(5):R135.

13. Harigai M, Takeuchi T, Tanaka Y, Matsubara T, Yamanaka H, Miyasaka N. Discontinuation of adalimumab treatment in rheumatoid arthritis patients after achieving low disease activity. Mod Rheumatol. 2012;22(6):81422.

14. Quinn MA, Conaghan PG, O'Connor PJ, Karim Z, Greenstein A, Brown A, et al. Very early treatment with infliximab in addition to methotrexate in early, poor-prognosis rheumatoid arthritis reduces magnetic resonance imaging evidence of synovitis and damage, with sustained benefit after infliximab withdrawal. Arthritis Rheum. 2005;52(1):27-35.

15. van den Broek M, Klarenbeek NB, Dirven L, van Schaardenburg D, Hulsmans HMJ, Kerstens PJSM, et al. Discontinuation of infliximab and potential predictors of persistent low disease activity in patients with early rheumatoid arthritis and disease activity score-steered therapy: subanalysis of the BeSt study. Ann Rheum Dis. 2011;70(8):1389-94.

16. Tanaka Y, Takeuchi T, Mimori T, Saito K, Nawata M, Kameda H, et al. Discontinuation of infliximab after attaining low disease activity in patients with rheumatoid arthritis: RRR (remission induction by Remicade in
RA) study. Ann Rheum Dis. 2010;69(7):128691.

17. Smolen JS, Emery P, Ferraccioli GF, Samborski W, Berenbaum F, Davies OR, et al. Certolizumab pegol in rheumatoid arthritis patients with low to moderate activity: the CERTAIN double-blind, randomised, placebo-controlled trial. Ann Rheum Dis. 2015;74(5):843-50.

18. van Herwaarden N, van der Maas A, Minten MJM, van den Hoogen FHJ, Kievit W, van Vollenhoven RF, et al. Disease activity guided dose reduction and withdrawal of adalimumab or etanercept compared with usual care in rheumatoid arthritis: open label, randomised controlled, noninferiority trial. BMJ. 2015;350:h1389.

19. Fautrel B, Pham T, Alfaiate T, Gandjbakhch F, Foltz V, Morel J, et al. Step-down strategy of spacing TNF-blocker injections for established rheumatoid arthritis in remission: results of the multicentre non-inferiority randomised open-label controlled trial (STRASS: Spacing of TNFblocker injections in Rheumatoid Arthritis St. Ann Rheum Dis. 2016;75(1):59-67.

20. Haschka J, Englbrecht M, Hueber AJ, Manger B, Kleyer A, Reiser M, et al. Relapse rates in patients with rheumatoid arthritis in stable remission tapering or stopping antirheumatic therapy: interim results from the prospective randomised controlled RETRO study. Ann Rheum Dis. 2016;75(1):45-51.

21. Botsios C, Furlan A, Ostuni R, Sfriso R, Todesco S, Punzi L. Effects of low-dose 
etanercept in maintaining DAS-remission previously achieved with standard-dose in patients with rheumatoid arthritis. Ann Rheum Dis. 2007;66:54-54.

22. Emery $P$, Hammoudeh $M$, FitzGerald $O$, Combe B, Martin Mola E, Bukowski J, et al. Assessing maintenance of remission with reduced dose etanercept plus methotrexate, methotrexate alone, or placebo in patients with early rheumatoid arthritis who achieved remission with etanercept and methotrextate: the prize study. Ann Rheum Dis. 2014;72(Suppl 3):A399-A399.

23. Kavanaugh A, Smolen JS. The when and how of biologic agent withdrawal in rheumatoid arthritis: learning from large randomised controlled trials. Clin Exp Rheumatol. 2013;31(4 Suppl 78):S19-21.

24. Kuijper TM, Lamers-Karnebeek FBG, Jacobs JWG, Hazes JMW, Luime JJ. Flare Rate in Patients with Rheumatoid Arthritis in Low Disease Activity or Remission When Tapering or Stopping Synthetic or Biologic DMARD: A Systematic Review. J Rheumatol. 2015;42(11):2012-22.

25. Saleem B, Keen $H$, Goeb V, Parmar R, Nizam S, Hensor EMA, et al. Patients with RA in remission on TNF blockers: when and in whom can TNF blocker therapy be stopped? Ann Rheum Dis. 2010;69(9):163642.

26. Hashimoto M, Furu M, Yamamoto W, Fujimura T, Hara R, Katayama M, et al. Factors associated with the achievement of biological disease-modifying antirheumatic drug-free remission in rheumatoid arthritis: the ANSWER cohort study. Arthritis Res Ther. 2018;20(1):165.

27. Centola M, Cavet G, Shen Y, Ramanujan S, Knowlton N, Swan KA, et al. Development of a multi-biomarker disease activity test for rheumatoid arthritis. PLoS One. 2013;8(4):e60635.

28. Curtis JR, van der Helm-van Mil AH, Knevel R, Huizinga TW, Haney DJ, Shen Y, et al. Validation of a novel multibiomarker test to assess rheumatoid arthritis disease activity. Arthritis Care Res (Hoboken). 2012;64(12):1794-803.

29. Eastman PS, Manning WC, Qureshi F, Haney D, Cavet G, Alexander C, et al. Characterization of a multiplex, 12-biomarker test for rheumatoid arthritis. J Pharm Biomed Anal. 2012;70:415-24.

30. Hirata S, Li W, Defranoux N, Cavet G, Bolce $\mathrm{R}$, Yamaoka $\mathrm{K}$, et al. A multi-biomarker disease activity score tracks clinical response consistently in patients with rheumatoid arthritis treated with different anti-tumor necrosis factor therapies: A retrospective observational study. Mod Rheumatol. 2015 May;25(3):344-9.

31. van der Helm-van Mil AHM, Knevel R, Cavet G, Huizinga TWJ, Haney DJ. An evaluation of molecular and clinical remission in rheumatoid arthritis by assessing radiographic progression. Rheumatology (Oxford). 2013;52(5):839-46.

32. Hambardzumyan K, Bolce R, Saevarsdottir S, Cruickshank SE, Sasso EH, Chernoff D, et al. Pretreatment multi-biomarker disease 
activity score and radiographic progression in early RA: results from the SWEFOT trial. Ann Rheum Dis. 2015;74(6):1102-9.

33. Li W, Sasso EH, van der Helm-van Mil AHM, Huizinga TWJ. Relationship of multibiomarker disease activity score and other risk factors with radiographic progression in an observational study of patients with rheumatoid arthritis. Rheumatology (Oxford). 2016;55(2):357-66.

34. Rech J, Hueber AJ, Finzel S, Englbrecht M, Haschka J, Manger B, et al. Prediction of disease relapses by multibiomarker disease activity and autoantibody status in patients with rheumatoid arthritis on tapering DMARD treatment. Ann Rheum Dis. 2015;75(9):1637-44.

35. Oderda GM, Lawless GD, Wright GC, Nussbaum SR, Elder R, Kim K, et al. The potential impact of monitoring disease activity biomarkers on rheumatoid arthritis outcomes and costs. Per Med. 2018;15(4):291-301.

36. Chatzidionysiou K, Turesson C, Teleman A, Knight A, Lindqvist $E$, Larsson $P$, et al. A multicentre, randomised, controlled, open-label pilot study on the feasibility of discontinuation of adalimumab in established patients with rheumatoid arthritis in stable clinical remission. RMD open. 2016;2(1):e000133.

37. Brocq O, Millasseau E, Albert C, Grisot C, Flory $\mathrm{P}$, Roux $\mathrm{C}-\mathrm{H}$, et al. Effect of discontinuing TNFalpha antagonist therapy in patients with remission of rheumatoid arthritis. Jt Bone Spine. 2009;76(4):350-5.
38. Kievit W, van Herwaarden $N$, van den Hoogen FH, van Vollenhoven RF, Bijlsma JW, van den Bemt BJ, et al. Disease activityguided dose optimisation of adalimumab and etanercept is a cost-effective strategy compared with non-tapering tight control rheumatoid arthritis care: analyses of the DRESS study. Ann Rheum Dis. 2016;75(11):1939-44.

39. Vanier A, Mariette X, Tubach F, Fautrel B, STRASS Study Group. Cost-Effectiveness of TNF-Blocker Injection Spacing for Patients with Established Rheumatoid Arthritis in Remission: An Economic Evaluation from the Spacing of TNF-Blocker Injections in Rheumatoid Arthritis Trial. Value Health. 2017;20(4):577-85.

40. Kobelt G. Treating to target with etanercept in rheumatoid arthritis: cost-effectiveness of dose reductions when remission is achieved. Value Health. 2014;17(5):537-44.

41. van der Maas A, Kievit W, van den Bemt BJF, van den Hoogen FHJ, van Riel PL, den Broeder AA. Down-titration and discontinuation of infliximab in rheumatoid arthritis patients with stable low disease activity and stable treatment: an observational cohort study. Ann Rheum Dis. 2012;71(11):1849-54.

42. Murphy $\mathrm{CL}$, Awan $\mathrm{S}$, Sullivan $\mathrm{MO}$, Chavrimootoo S, Bannon C, Martin L, et al. Major cost savings associated with biologic dose reduction in patients with inflammatory arthritis. Ir Med J. 2015;108(1):19-21.

43. ten Klooster PM, Veehof MM, Taal E, van Riel PL, van de Laar MA. Changes in 
priorities for improvement in patients with rheumatoid arthritis during 1 year of antitumour necrosis factor treatment. Ann Rheum Dis. 2007;66(11):1485-90.

44. Buchbinder R, Bombardier C, Yeung $M$, Tugwell P. Which outcome measures should be used in rheumatoid arthritis clinical trials? Clinical and quality-of-life measures' responsiveness to treatment in a randomized controlled trial. Arthritis Rheum. 1995;38(11):1568-80.

45. Anderson JJ, Chernoff MC. Sensitivity to change of rheumatoid arthritis clinical trial outcome measures. J Rheumatol. 1993;20(3):535-7.

46. Verhoeven AC, Boers M, van Der Linden S. Responsiveness of the core set, response criteria, and utilities in early rheumatoid arthritis. Ann Rheum Dis. 2000;59(12):96674.

47. Russell AS, Conner-Spady B, Mintz A, Maksymowych WP. The responsiveness of generic health status measures as assessed in patients with rheumatoid arthritis receiving infliximab. J Rheumatol. 2003;30(5):941-7.

48. Tugwell P, Wells $G$, Strand $V$, Maetzel A, Bombardier C, Crawford B, et al. Clinical improvement as reflected in measures of function and health- related quality of life following treatment with leflunomide compared with methotrexate in patients with rheumatoid arthritis: Sensitivity and relative efficiency to detect a treatment. Arthritis Rheum. 2000;43(3):506-14.

49. Cohen SB, Strand V, Aguilar D, Ofman
JJ. Patient- versus physician-reported outcomes in rheumatoid arthritis patients treated with recombinant interleukin-1 receptor antagonist (anakinra) therapy. Rheumatol. 2004;43(6):704-11.

50. Ranganath VK, Yoon J, Khanna D, Park GS, Furst DE, Elashoff DA, et al. Comparison of composite measures of disease activity in an early seropositive rheumatoid arthritis cohort. Ann Rheum Dis. 2007;66(12):163340.

51. Aletaha D, Nell VP, Stamm T, Uffmann M, Pflugbeil S, Machold K, et al. Acute phase reactants add little to composite disease activity indices for rheumatoid arthritis: validation of a clinical activity score. Arthritis Res Ther. 2005;7(4):R796-806.

52. Aletaha D, Landewe $\mathrm{R}$, Karonitsch $\mathrm{T}$, Bathon J, Boers M, Bombardier C, et al. Reporting disease activity in clinical trials of patients with rheumatoid arthritis: EULAR/ ACR collaborative recommendations. Ann Rheum Dis. 2008;67(10):1360-4. 
NEDERLANDSE SAMENVATING 
ADDENDUM 


\section{ACKNOWLEDGEMENTS/DANKWOORD}

Aan het einde van een proefschrift is een woord van dank op zijn plaats. Zeker als het proefschrift gebaseerd is op een groot onderzoek als de POEET studie.

De POEET studie is net als de ziekte Reumatoide Artritis gekenmerkt door ups en downs (remissies en exacerbaties). Omdat ik me bewust ben zelf te hebben bijgedragen aan de downs ben ik erg dankbaar voor de velen die uiteindelijk bijgedragen hebben aan het succes van het POEET project.

In de eerste plaats ben ik vooral erg veel dank verschuldigd aan de bijna duizend patiënten die bereid zijn geweest aan de POEET studie mee te doen. Velen deden vooral mee uit het algemene belang omdat hun persoonlijke belang, remissie van hun RA, reeds behaald was door succesvolle medicamenteuze therapie. Aangezien ik al deze patiënten niet bij naam kan noemen, zal ik uit respect voor hen ook hierna geen mensen persoonlijk benoemen. Alle patiënten die belangstelling toonden voor de POEET studie en vooral aan allen die uiteindelijk ook hebben mee gedaan hartelijk dank. Uw tijd, energie en belangeloze inzet is van onschatbare waarde geweest.

Het ministerie van Volksgezondheid, Welzijn en Sport en mijn primaire begeleiders hebben de noodzaak van een TNFi stopstudie gezien en het initiatief genomen. Tezamen met het Zorginstituut Nederland werd de POEET studie mogelijk gemaakt. Ongeveer de helft van de collega Nederlandse reumatologen en hun ziekenhuizen hebben hun tijd, energie en creativiteit gegeven om de uitvoering van de studie mogelijk te maken. Het belang van de studie werd door allen erkend waardoor de studie onder een reële kostprijs kon worden uitgevoerd. Mijn rechtstreekse collega's, zowel in Enschede, Nijmegen, Leuven en uiteindelijk definitief Enschede, hebben me gestimuleerd en de ruimte gegeven om op basis van de POEET studie een proefschrift te schrijven. Aan allen ben in grote dank verschuldigd en u allen zal ik altijd dankbaar blijven.

Hoewel ik geboren, opgegroeid en opgeleid ben in mijn moederland Iran, dat ik heb moeten verlaten, heb ik een nieuwe start in Nederland gemaakt. Nederland heeft mij steeds weer nieuwe kansen gegeven waardoor ik enorm dankbaar ben dat ik Nederlander ben. In een dankwoord wil je niemand vergeten, mochten er mensen zijn die zich ondanks mijn intenties, niet herkennen in bovenstaande woorden van dank, weet dat ik echt iedereen die bijgedragen heeft aan het succes van het POEET project en dit proefschrift voor altijd dankbaar zal zijn. 
ADDENDUM

Aan het einde van mijn dankwoord wil ik toch mijn allerliefste benoemen: mijn lieve dochter, Helena, jij bent mijn trots, mijn inspiratie en mijn kracht!

Marjan Ghiti Moghadam. 


\section{ABOUT THE AUTHOR}

Marjan Ghiti Moghadam was born on the $3^{\text {th }}$ of July 1969 in Tehran, Iran. In 1987 she finished secondary education in Iran. In 1995 she received her degree in medicine (general practitioner) at the Medical University in Tehran. Immediately thereafter, she started working as a general practitioner in Northern Iran (Astara). After 4 years she returned to Tehran. In 2000 she immigrated to The Netherlands, followed a special program for foreign doctors at the faculty of Medical Sciences of the Radboud University Nijmegen and in 2007 she graduated as a Medical Doctor. Thereafter she started as a resident Internal Medicine in the Deventer Hospital and in 2011 she moved to Enschede for her residency Rheumatology in Almelo (Ziekenhuisgroep Twente) and Enschede (Medisch Spectrum Twente). In 2014 she graduated as a Rheumatologist. At the end of her residency she also started her PhD project, supervised by prof. dr. Mart van de Laar, dr. Harald Vonkeman and dr. Tim Jansen, on the stopping of tumor necrosis factor inhibitors in well controlled rheumatoid arthritis patients (the POET study) at the University of Twente. In 2014 she started working as a Rheumatologist at the Department of Rheumatology and Immunology of the Radboud University Medical Center Nijmegen. In 2015 she temporarily left The Netherlands to continue her career in Belgium as a Rheumatologist in University Hospital Leuven and Diest Medical Center. In 2017 she decided to return to The Netherlands (Enschede), to work as a Rheumatologist at the Arthritis Center Twente of the Medisch Spectrum Twente (MST). 
ADDENDUM 


\section{LIST OF PUBLICATIONS}

-Ghiti Moghadam M, Vonkeman HE, Ten Klooster PM, Tekstra J, van Schaardenburg D, Starmans-Kool M, et al. Stopping Tumor Necrosis Factor Inhibitor Treatment in Patients With Established Rheumatoid Arthritis in Remission or With Stable Low Disease Activity: A Pragmatic Multicenter, Open-Label Randomized Controlled Trial. Arthritis Rheumatol (Hoboken, NJ). 2016;68:1810-7.

-Ghiti Moghadam M, Lamers-Karnebeek FBG, Vonkeman HE, Ten Klooster PM, Tekstra J, Schilder AM, Visser H, Sasso EH, Chernoff D, Lems WF, van Schaardenburg DJ, Landewe R, Bernelot Moens HJ, Radstake TRDJ, van Riel PLCM, van de Laar MAFJ, Jansen TL; Dutch National POET Collaboration.Multi-biomarker disease activity score as a predictor of disease relapse in patients with rheumatoid arthritis stopping TNF inhibitor treatment. PLoS One. 2018 May 23;13(5):e0192425.

-Ghiti Moghadam M, Ten Klooster PM, Vonkeman HE, Kneepkens EL, Klaasen R, Stolk JN, Tchetverikov I, Vreugdenhil SA, van Woerkom JM, Goekoop-Ruiterman YPM, Landewé RBM, van Riel PLCM, van de Laar MAFJ, Jansen TL; Dutch National POET Collaboration. Impact of Stopping Tumor Necrosis Factor Inhibitors on Rheumatoid Arthritis Patients' Burden of Disease. Arthritis Care Res (Hoboken). 2018 Apr;70(4):516-524.

-Tran-Duy A, Ghiti Moghadam M, Oude Voshaar MAH, Vonkeman HE, Boonen A, Clarke P, McColl G, Ten Klooster PM, Zijlstra TR, Lems WF, Riyazi N, Griep EN, Hazes JMW, Landewé R, Bernelot Moens HJ, van Riel PLCM, van de Laar MAFJ, Jansen TL; Dutch National POET Collaboration. An Economic Evaluation of Stopping Versus Continuing Tumor Necrosis Factor Inhibitor Treatment in Rheumatoid Arthritis Patients With Disease Remission or Low Disease Activity: Results From a Pragmatic Open-Label Trial. Arthritis Rheumatol. 2018 Oct;70(10):1557-1564.

-Martijn A.H. Oude Voshaar, PhD; Marjan Ghiti Moghadam, MD,; Harald E. Vonkeman, MD, PhD; Peter M. ten Klooster, PhD; Dirkjan van Schaardenburg, MD, PhD; Janneke Tekstra, MD, PhD; H. Visser, MD, PhD; Mart A.F.J. van de Laar, Tim L. Jansen, MD, PhD. Measuring disease exacerbation and flares in rheumatoid arthritis: Comparison of commonly used disease activity indices\& individual measures. J Rheumatol. 2017 Aug;44(8):1118-1124. doi: 10.3899/jrheum.160915. Epub 2017 May 15.

-Lamers-Karnebeek FB, Luime JJ, Ten Cate DF, Teerenstra S, Swen NWAA, Gerards AH, Hendrikx J, van Rooyen EM, Voorneman R, Haagsma C, Basoski N, de Jager M, Ghiti 
Moghadam M, Efde MN, Goekoop-Ruiterman YPM, van Riel PLCM, Jacobs JWG, Jansen TL. Limited value for ultrasonography in predicting flare in rheumatoid arthritis patients with Iow disease activity stopping TNF inhibitors. Rheumatology (Oxford). 2017 Sep 1;56(9):15601565. doi: 10.1093/rheumatology/kex184. 


Marjan Chiti Moghadam is a rheumatologist at the Medisch Spectrum Twente.

The research for the phd was performed at the Arthritis Center

Twente of the Medisch Spectrum Twente hospital en Twente University, The Netherlands. It focused on the efficacy en safety van stopping Tumor Necrosis Factor inhibitors in well controlled rheumatoid arthritis patients. 